\title{
Intermolecular Phosphite-Mediated Radical Desulfurative Alkene Alkylation Using Thiols
}

John M. Lopp and Valerie A. Schmidt*

University of California San Diego, Department of Chemistry and Biochemistry, 9500 Gilman Drive, La Jolla, CA 92093

vschmidt@ucsd.edu

Supplementary Information 


\section{Table of Contents}

A. General Considerations $\quad$ S3

B. Extended Optimization Studies

S4

C. Preparation of Alkenes

S5

D. Preparation of Thiols

S8

E. General Alkylation Procedures $\quad$ S12

F. Characterization of Alkylation Products in Alkene Scope $\mathbf{S 1 2}$

G. Characterization of Alkylation Products in Thiol Scope S32

$\begin{array}{ll}\text { H. References } & \text { S39 }\end{array}$

I. ${ }^{1} \mathrm{H}$ and ${ }^{13} \mathrm{C}$ NMR Spectra $\quad \$ 40$ 
A. General Considerations. All air- and moisture-sensitive manipulations were carried out using standard high vacuum line with standard Schlenk line techniques. All solvents or reagents were purchased from commercial sources and were used as received without further purification unless specified.

${ }^{1} \mathrm{H}$ and ${ }^{13} \mathrm{C}$ NMR were recorded on a Brucker AVA $300 \mathrm{MHz}$ or Varian Unity/lnova $500 \mathrm{MHz}$ spectrometer. All chemical shifts are reported relative to chemical shifts of the protio-solvent. GC analyses were performed using an Agilent Technologies 7890B gas chromatograph equipped with an Agilent 7693 autosampler and Agilent HP-5 capillary column (30 m x 0.320 mm x $250 \mu \mathrm{m}$ ). Standard method parameters: $1.2 \mathrm{~mL} / \mathrm{min}$ flow rate with oven program $80-250{ }^{\circ} \mathrm{C}$ with a ramp rate of $25{ }^{\circ} \mathrm{C} / \mathrm{min}$ and hold time of 8.7 minutes at $250^{\circ} \mathrm{C}$. Low-resolution mass spectra were measured using a Thermo LCQdeca APCI-MS and high-resolution mass spectra were obtained using an Agilent 6230 AccurateMass TOFMS. Elemental analysis was provided by Robertson Microlit Laboratories. Syringe pump used in the general alkylation procedure was a NE-1000 Programmable Single Syringe Pump. 


\section{B. Extended Optimization Studies}<smiles>COc1ccc(C(=O)CS)cc1</smiles>

[0.16 mmol] added at $2.3 \mu \mathrm{L} / \mathrm{min}$

\begin{tabular}{|c|c|c|}
\hline entry & Deviation from above conditions & Yield 2a \\
\hline 1 & none & $93 \%$ \\
\hline 2 & $\mathrm{PPh}_{3}$ instead of $\mathrm{P}(\mathrm{OEt})_{3}$ & $90 \%$ \\
\hline 3 & $\mathrm{P}(\mathrm{OMe})_{3}$ instead of $\mathrm{P}(\mathrm{OEt})_{3}$ & $40 \%$ \\
\hline 4 & $\mathrm{P}\left(\mathrm{O}^{\mathrm{i}} \mathrm{Pr}\right)_{3}$ instead of $\mathrm{P}(\mathrm{OEt})_{3}$ & $59 \%$ \\
\hline 5 & $\mathrm{P}(\mathrm{OPh})_{3}$ instead of $\mathrm{P}(\mathrm{OEt})_{3}$ & $33 \%$ \\
\hline 6 & Dilauroyl Peroxide instead of AIBN & $50 \%$ \\
\hline 7 & $\left({ }^{t} \mathrm{BuON}\right)_{2}$ instead of AIBN & not detected $^{a}$ \\
\hline 8 & MeCN solvent & $46 \%$ \\
\hline 9 & DCE solvent & $44 \%$ \\
\hline 10 & $1,1,1$-Trifluorotoluene solvent & $81 \%^{b}$ \\
\hline 11 & Toluene solvent & $82 \%{ }^{b}$ \\
\hline 12 & Ethanol solvent & $92 \%{ }^{b}$ \\
\hline 13 & Cyclohexane solvent & $73 \%{ }^{b}$ \\
\hline
\end{tabular}

${ }^{a} 1$ and ( $\left.{ }^{t} \mathrm{BuON}\right)_{2}$ added in one portion. ${ }^{b}$ Reactions carried out using 3 equiv of NBVE.

Scheme S1. Direct comparison of tert-butyl isocyanide and triethyl phosphite in reductive alkylations. A. Reductive alkylation using tert-butyl isocyanide

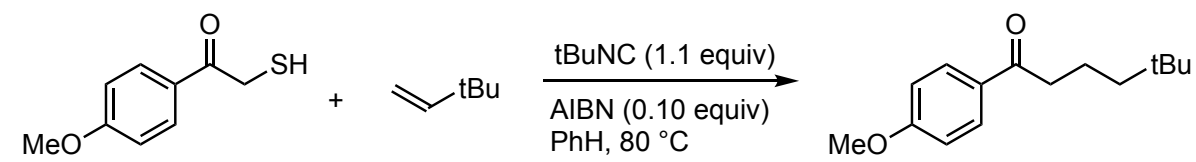

[0.1 mmol] added over $1 \mathrm{~h}$

10 equiv

$13 \%$ yield

a Conditions as described by: L. Benati, R. Leardini, M. Minozzi, D. Nanni, R. Scialpi, P. Spangnolo,

S. Strazzari, G. Zanardi, Angew. Chem. Int. Ed. 2004, 43, 3598-3601

B. Reducitve alkylation using triethyl phosphite (this work)

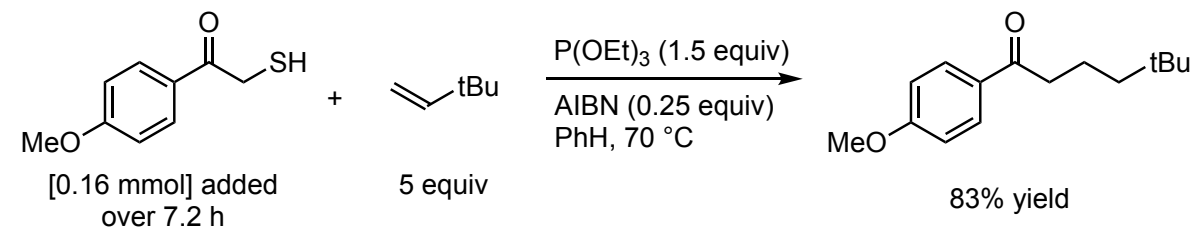




\section{Preparation of Alkenes}

$$
\curvearrowright \text { OAc }
$$

But-3-enyl acetate was prepared according to a previously reported procedure and spectra were in accordance with literature. ${ }^{1}$

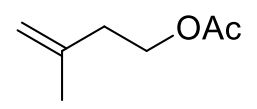

3-Methylbut-3-enyl acetate was prepared according to a previously reported procedure ${ }^{1}$ and spectra were in accordance with literature. ${ }^{2}$<smiles>C=CCc1ccccc1OC(C)=O</smiles>

2-Allylphenyl acetate was prepared according to a previously reported procedure and spectra were in accordance with literature. ${ }^{3}$<smiles>C=CCOC(=O)N1CCOCC1</smiles>

Allyl piperidine-1-carboxylate was prepared in a similar manner to a previously reported literature. ${ }^{4}$ To a stirring solution of morpholine $(0.50 \mathrm{~mL}, 5.7 \mathrm{mmol})$, triethylamine $(1.25$ equiv, $1.0 \mathrm{~mL}, 7.2 \mathrm{mmol})$, and THF $(29 \mathrm{~mL})$ in a round bottomed flask with ice bath was added dropwise a solution of allyl chloroformate ( 1.25 equiv, $0.76 \mathrm{~mL}, 7.2 \mathrm{mmol}$ ) dropwise. The reaction was let come to room temperature and, when no starting material remained, the mixture was quenched with $5 \mathrm{~mL}$ saturated sodium bicarbonate and let stir for 10 minutes. The reaction mixture was then partitioned between 100 $\mathrm{mL} \mathrm{DCM}$ and $50 \mathrm{~mL}$ saturated sodium bicarbonate and the organic phase was washed with $50 \mathrm{~mL}$ saturated ammonium chloride, $50 \mathrm{~mL}$ of brine, and then dried with sodium sulfate. After concentrating en vacuo the residue was purified by chromatography (30\% ethyl acetate/hexanes) and isolated as a clear oil (912 mg, 95\% yield).

${ }^{1} \mathrm{H}-\mathrm{NMR}\left(500 \mathrm{MHz} ; \mathrm{CDCl}_{3}\right): \delta 5.94-5.82(\mathrm{~m}, 1 \mathrm{H}), 5.25$ (ddd, J = 14.1, 3.7, $\left.1.9 \mathrm{~Hz}, 1 \mathrm{H}\right), 5.19-5.13$ $(\mathrm{m}, 1 \mathrm{H}), 4.58-4.52(\mathrm{~m}, 2 \mathrm{H}), 3.56-3.64(\mathrm{~s}, 4 \mathrm{H}), 3.49-3.36(\mathrm{~m}, 4 \mathrm{H})$.

${ }^{13}$ C-NMR (126 MHz; $\left.\mathrm{CDCl}_{3}\right): \delta$ 155.20, 132.95, 117.69, 66.74, 66.62, 66.24, 44.33, 43.94.

HRMS (APCI) Calc. for $\left[\mathrm{C}_{8} \mathrm{H}_{13} \mathrm{NO}_{3}+\mathrm{H}\right]^{+}=172.0973$, Found $\left[\mathrm{C}_{8} \mathrm{H}_{13} \mathrm{NO}_{3}+\mathrm{H}\right]^{+}=172.0970$

$\mathbf{R}_{\mathbf{f}}=0.3$ in $20 \%$ ethyl acetate/hexanes 


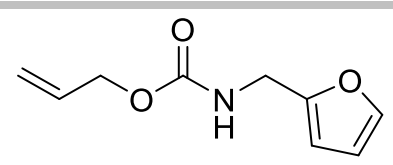

Allyl (furan-2-yl-methyl)carbamate was prepared in a similar manner to Allyl piperidine-1carboxylate. Product was purified by column chromatography (15\% ethyl acetate/hexanes) and isolated as a colorless oil.

${ }^{1} \mathrm{H}-N M R\left(500 \mathrm{MHz} ; \mathrm{CDCl}_{3}\right): \delta 7.30$ (dd, J = 1.8, $\left.0.8 \mathrm{~Hz}, 1 \mathrm{H}\right), 6.26(\mathrm{dd}, \mathrm{J}=2.8,1.9 \mathrm{~Hz}, 1 \mathrm{H}), 6.18(\mathrm{~s}$, $1 \mathrm{H}), 5.93-5.79(\mathrm{~m}, 1 \mathrm{H}), 5.21(\mathrm{dd}, \mathrm{J}=46.3,13.8 \mathrm{~Hz}, 4 \mathrm{H}), 4.53(\mathrm{~s}, 2 \mathrm{H}), 4.31(\mathrm{~d}, \mathrm{~J}=5.4 \mathrm{~Hz}, 4 \mathrm{H})$.

${ }^{13}$ C-NMR (126 MHz; $\left.\mathrm{CDCl}_{3}\right): \delta$ 156.17, 151.68, 142.28, 132.84, 117.88, 110.48, 107.32, 65.85, 38.12.

HRMS (APCI) Calc. for $\left[\mathrm{C}_{9} \mathrm{H}_{11} \mathrm{NO}_{3}+\mathrm{Na}\right]^{+}=204.0636$, Found $\left[\mathrm{C}_{9} \mathrm{H}_{11} \mathrm{NO}_{3}+\mathrm{Na}\right]^{+}=204.0633$

$\mathbf{R}_{\mathbf{f}}=0.3$ in $15 \%$ ethyl acetate/hexanes<smiles>C=CCOC(=O)NCc1cccs1</smiles>

Allyl (thiophen-2-ylmethyl)carbamate was prepared in a similar manner Allyl piperidine-1carboxylate. Product was purified by column chromatography (20\% ethyl acetate/hexanes) and isolated as a colorless oil.

${ }^{1}$ H-NMR (300 MHz; $\left.\mathrm{CDCl}_{3}\right): \delta 7.20-7.15(\mathrm{~m}, 1 \mathrm{H}), 6.97-6.85(\mathrm{~m}, 2 \mathrm{H}), 5.87(\mathrm{ddd}, \mathrm{J}=11.0,7.9,3.8$ $\mathrm{Hz}, 1 \mathrm{H}), 5.39-5.11(\mathrm{~m}, 3 \mathrm{H}), 4.67-4.39(\mathrm{~m}, 4 \mathrm{H})$.

${ }^{13} \mathrm{C}-\mathrm{NMR}\left(126 \mathrm{MHz} ; \mathrm{CDCl}_{3}\right): \delta$ 156.08, 141.41, 141.37, 132.81, 126.92, 125.80, 125.16, 117.83, 65.80, 39.89 .

HRMS (APCI) Calc. for $\left[\mathrm{C}_{9} \mathrm{H}_{11} \mathrm{NO}_{2} \mathrm{~S}+\mathrm{H}\right]^{+}=198.0588$, Found $\left[\mathrm{C}_{18} \mathrm{H}_{20} \mathrm{O}_{3}+\mathrm{H}\right]^{+}=198.0585$

$\mathbf{R}_{\mathbf{f}}=0.3$ in $20 \%$ ethyl acetate/hexanes

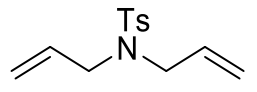

$\boldsymbol{N}, \boldsymbol{N}$-Diallyl para-toluenesulfonamide was prepared according to a previously reported procedure and spectra were in accordance with literature. ${ }^{5}$ 


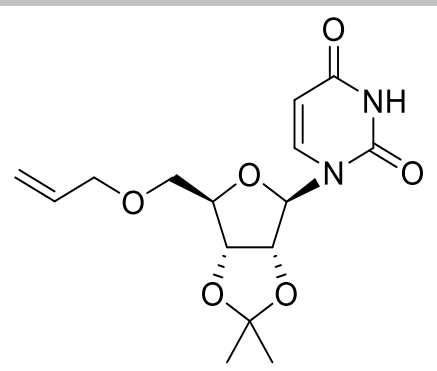

5'-allyl-2',3'-Isopropylideneuridine was prepared according to a previously reported procedure and spectra were in accordance with literature. ${ }^{6}$

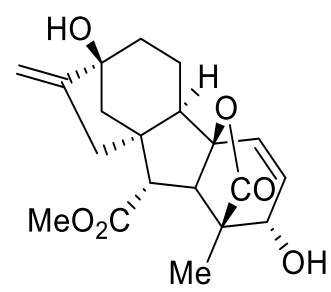

Gibberellic acid methyl ester was prepared according to a previously reported procedure and spectra were in accordance with literature. ${ }^{7}$<smiles>C=C1O[C@H](OC)[C@H](OC(C)=O)[C@H](OC(C)=O)[C@@H]1OC(C)=O</smiles>

Methyl-2,3,4-tri-O-acetyl-6-desoxy-a-D-xylo-hex-5-enopyranoside was synthesized in three steps from Methyl $\alpha$-D-glucopyranoside as reported in a literature procedure. ${ }^{8}$

${ }^{1} \mathrm{H}-\mathrm{NMR}\left(300 \mathrm{MHz} ; \mathrm{CDCl}_{3}\right): \delta 5.49-5.38(\mathrm{~m}, 2 \mathrm{H}), 4.95$ (dd, J = 3.8, $\left.1.8 \mathrm{~Hz}, 2 \mathrm{H}\right), 4.75$ (d, J = $1.8 \mathrm{~Hz}$, $1 \mathrm{H}), 4.56(\mathrm{~d}, \mathrm{~J}=1.6 \mathrm{~Hz}, 1 \mathrm{H}), 3.40(\mathrm{~s}, 3 \mathrm{H}), 2.08(\mathrm{~s}, 3 \mathrm{H}), 2.04(\mathrm{~s}, 3 \mathrm{H}), 1.98(\mathrm{~s}, 3 \mathrm{H})$.

${ }^{13}$ C-NMR (126 MHz; $\left.\mathrm{CDCl}_{3}\right): \delta$ 170.30, 169.99, 169.68, 149.82, 97.98, 97.57, 70.76, 69.78, 69.50, $55.71,20.89,20.86,20.84$.

HRMS (APCI) Calc. for $\left[\mathrm{C}_{13} \mathrm{H}_{18} \mathrm{O}_{8}+\mathrm{Na}\right]^{+}=325.0899$, Found $\left[\mathrm{C}_{18} \mathrm{H}_{20} \mathrm{O}_{3}+\mathrm{H}\right]^{+}=325.0893$ 


\section{Preparation of Thiols}

General Procedure for the Bromination of Ketones - A sample procedure for the synthesis of a-bromo ketones is described: A round bottomed flask with $p$-fluoro-acetophenone $(1.5 \mathrm{~mL}, 12.0 \mathrm{mmol})$ in $\mathrm{CH}_{2} \mathrm{Cl}_{2}(120 \mathrm{~mL})$ under nitrogen was connected by hose to a trap of saturated sodium bicarbonate. Bromine (1.1 equiv, $0.70 \mathrm{~mL}, 2.4 \mathrm{mmol}$ ) was added dropwise to the reaction. The reaction was followed by ${ }^{1} \mathrm{H}-\mathrm{NMR}$ analysis of aliquots. Once starting material was judged to be consumed, $20 \mathrm{~mL}$ of a saturated sodium thiosulfate aqueous solution and $20 \mathrm{~mL}$ of saturated bicarbonate (aqueous) was added and the reaction mixture let stir for 15 minutes. Then, the reaction mixture was diluted with $\mathrm{CH}_{2} \mathrm{Cl}_{2}(100 \mathrm{~mL})$ and sequentially washed with water $(80 \mathrm{~mL})$, brine $(80 \mathrm{~mL})$, and dried over sodium sulfate before concentration en vacuo. After purification by flash chromatography (10\% ethyl acetate/hexanes) obtained as an orange oil ( $1.2 \mathrm{~g}, 45 \%$ yield $)$

General Procedure for the Preparation of Thioesters - A sample procedure for the synthesis of thioesters from alkyl chloride or bromides is described: A round bottomed flask with starting $p$-methoxya-bromo acetophenone $(2.02 \mathrm{~g}, 8.73 \mathrm{mmol})$ in acetone $(87 \mathrm{~mL})$ was added potassium thioacetate $(1.1$ equiv, $1.10 \mathrm{~g}, 9.60 \mathrm{mmol}$ ), which was let stir at room temperature until no starting material remained by TLC. The reaction mixture was filtered, concentrated en vacuo, and the resulting residue was partitioned between water $(150 \mathrm{~mL})$ and $\mathrm{CH}_{2} \mathrm{Cl}_{2}(400 \mathrm{~mL})$. The organic layer was washed with brine $(150 \mathrm{~mL})$ and dried with sodium sulfate and then concentrated to give the desired thioester as an orange oil ( $1.8 \mathrm{~g}, 90 \%$ yield) after purification by flash chromatography (15\% ethyl acetate/hexanes).

General Procedure for Synthesis of Thiols- A sample procedure for the deprotection of thioesters is described: To a round bottomed flask equipped with ice bath was added $p$-methoxy-2-thioacetylacetophenone $(2.49 \mathrm{~g}, 11.1 \mathrm{mmol})$ in acetonitrile $(110 \mathrm{~mL})$. A $35 \%$ aqueous solution of hydrazine hydrate (2.5 equiv, $2.5 \mathrm{~mL}, 27.7 \mathrm{mmol}$ ) was added dropwise. When no starting material remained by TLC (visualized with $p$-anisaldehyde stain) the reaction was quenched with $10 \mathrm{~mL}$ of $1 \mathrm{M} \mathrm{HCl}$ and let stir for 15 minutes. The reaction mixture was partitioned between $\mathrm{CH}_{2} \mathrm{Cl}_{2}(150 \mathrm{~mL})$ and $1 \mathrm{M} \mathrm{HCl}(100$ $\mathrm{mL})$. The organic phase was washed with brine $(80 \mathrm{~mL})$ and dried over sodium sulfate, then activated charcoal was added and the mixture filtered. Concentration en vacuo gave a residue which was purified by flash chromatography (15\% ethyl acetate/hexanes) to give desired thiol that crystallized in the fridge overnight as a tan solid (694 $\mathrm{mg}, 35 \%$ yield).<smiles>COc1ccc(C(=O)CS)cc1</smiles>

4'-methoxy-2-mercapto-acetophenone (1) was prepared from commercially available $p$-methoxy- $\alpha$ bromo-acetophenone using the general procedures above as a tan solid. The ${ }^{1} \mathrm{H}-\mathrm{NMR}$ spectrum matches that of previously reported literature. ${ }^{9}$ 
<smiles>O=C(CS)c1ccc(F)cc1</smiles>

4'-fluoro-2-mercapto-acetophenone was prepared from commercially available $p$-fluoroacetophenone via the general procedures above as a yellow solid. ${ }^{1} \mathrm{H}-\mathrm{NMR}$ spectrum matches that of previously reported literature. $^{9}$<smiles>O=C(CS)c1ccccc1</smiles>

2-mercapto-acetophenone was prepared from commercially available $\alpha$-bromo acetophenone using the general procedures above as a yellowish oil. The ${ }^{1} \mathrm{H}-\mathrm{NMR}$ spectrum matches that of previously reported literature. ${ }^{9}$

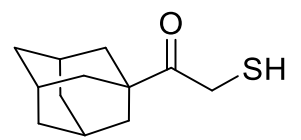

Methylmercapto adamantyl ketone was prepared from commercially available a-bromo methyl adamantly ketone using the general procedures above. Obtained as a white solid.

Analytical data:

${ }^{1} \mathrm{H}-\mathrm{NMR}\left(300 \mathrm{MHz} ; \mathrm{CDCl}_{3}\right): \delta 3.51(\mathrm{~d}, \mathrm{~J}=6.7 \mathrm{~Hz}, 1 \mathrm{H}), 2.08-1.97(\mathrm{~m}, 2 \mathrm{H}), 1.82(\mathrm{~d}, \mathrm{~J}=2.7 \mathrm{~Hz}, 3 \mathrm{H})$, $1.70(\mathrm{q}, \mathrm{J}=12.3 \mathrm{~Hz}, 3 \mathrm{H}), 1.53(\mathrm{~s}, 1 \mathrm{H})$.

${ }^{13} \mathrm{C}-\mathrm{NMR}\left(126 \mathrm{MHz} ; \mathrm{CDCl}_{3}\right): \delta 46.73,38.58,36.53,30.06,27.96$.

LRMS (APCI) Calc. for $\left[\mathrm{C}_{12} \mathrm{H}_{18} \mathrm{OS}+\mathrm{C}_{12} \mathrm{H}_{18} \mathrm{OS}-\mathrm{H}\right]^{+}=419.23$, Found $=419.21$ (observed as the disulfide)

HRMS (ESI) Calc. for $\left[\mathrm{C}_{24} \mathrm{H}_{36} \mathrm{O}_{2} \mathrm{~S}_{2}+\mathrm{H}\right]^{+}=419.2073$, Found $=419.2071$ (observed as the disulfide)

$\mathbf{R}_{\mathbf{f}}=0.7$ in $15 \%$ ethyl acetate/hexanes<smiles>O=C(CS)OCc1ccccc1</smiles>

benzyl 2-mercaptoacetate was prepared according to a previous literature procedure. ${ }^{1} \mathrm{H}-\mathrm{NMR}$ spectrum matches that of previously reported literature. ${ }^{10}$ 


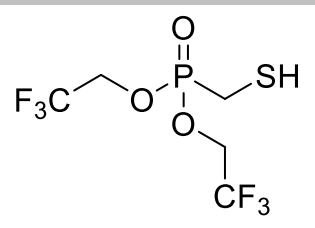

Bis(2-trifluoro)ethyl methylmercaptophosphonate was prepared from commercially available chloromethylphosphonic dichloride using known procedures. ${ }^{11}$ The intermediate O,O-bis(trifluoroethyl) chloromethylphosphonate was converted to the thioester using the procedures above. The free thiol was prepared as follows: To a $20 \mathrm{~mL}$ scintillation vial of 2,2,2-trifluoroethanol (10 mL) was added O,Obis(trifluoroethyl) thioacetylmethylphosphonate $(1.5 \mathrm{mmol})$ and hydrochloric acid $(0.6 \mathrm{~mL}, 4.5$ equiv). The reaction was refluxed for three days and evaporated en vacuo and used without further purification. The final product and intermediate phosphonates hydrolyzed readily and was not stable to silica. Obtained as a red oil (450 $\mathrm{mg}, 96 \%$ yield).

Analytical data:

${ }^{1} \mathrm{H}-\mathrm{NMR}\left(300 \mathrm{MHz} ; \mathrm{CDCl}_{3}\right): \delta 4.52-4.33(\mathrm{~m}, 1 \mathrm{H}), 2.83(\mathrm{dd}, \mathrm{J}=13.1,8.6 \mathrm{~Hz}, 1 \mathrm{H}), 1.88$ (td, J = 8.6, 7.0 $\mathrm{Hz}, 1 \mathrm{H})$.

${ }^{13} \mathrm{C}-\mathrm{NMR}\left(126 \mathrm{MHz} ; \mathrm{CDCl}_{3}\right): \delta 124.71$ (q), 63.33 (dq), 18.51, 17.85 (d)

${ }^{19}$ F-NMR $\left(126 \mathrm{MHz} ; \mathrm{CDCl}_{3}\right): \delta 75.38$

${ }^{31}$ P-NMR (126 MHz; $\left.\mathrm{CDCl}_{3}\right): \delta 27.83$ (d)

LRMS (APCI) Calc. for $\left[\mathrm{C}_{14} \mathrm{H}_{18} \mathrm{~F}_{6} \mathrm{O}_{3} \mathrm{PS}+\mathrm{H}\right]^{+}=292.98$, Found $=293.05$<smiles>CCN(CC)C(=O)CS</smiles>

Diethyl acetamide was prepared from 2-chloro-N,N-diethylacetamide by a known procedure. ${ }^{12}$ The thiol was prepared from the intermediate chloride using the procedures above. Obtained in $90 \%$ yield as a yellow oil.

Analytical data:

${ }^{1} \mathrm{H}-\mathrm{NMR}\left(300 \mathrm{MHz} ; \mathrm{CDCl}_{3}\right): \delta 3.44-3.19(\mathrm{~m}, 6 \mathrm{H}), 2.15(\mathrm{~d}, \mathrm{~J}=7.4 \mathrm{~Hz}, 1 \mathrm{H}), 1.18(\mathrm{t}, \mathrm{J}=7.2 \mathrm{~Hz}, 3 \mathrm{H})$, $1.10(\mathrm{t}, \mathrm{J}=7.1 \mathrm{~Hz}, 3 \mathrm{H})$

${ }^{13}$ C-NMR (126 MHz; $\left.\mathrm{CDCl}_{3}\right): \delta$ 169.09, 42.71, 40.89, 26.37, 26.36, 14.58, 12.96.

LRMS (APCI) Calc. for $\left[\mathrm{C}_{6} \mathrm{H}_{13} \mathrm{NOS}+\mathrm{H}^{+}=148.08\right.$, Found $=148.13$

HRMS (APCI) Calc. for $\left[\mathrm{C}_{12} \mathrm{H}_{24} \mathrm{~N}_{2} \mathrm{O}_{2} \mathrm{~S}_{2}+\mathrm{H}\right]^{+}=293.1352$, Found $=293.1409$

$\mathbf{R}_{\mathbf{f}}=0.4$ in $70 \%$ ethyl acetate/hexanes 


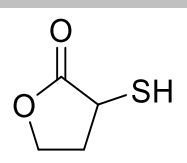

a-mercapto-y-butyrolactone was prepared from a-bromo-y-butyrolactone using the general procedures above as a clear oil. The ${ }^{1} \mathrm{H}-\mathrm{NMR}$ spectrum matches that of previously reported literature. ${ }^{13}$ 


\section{E. General Alkylation Procedure}

General Alkylation Procedure Desulfurative Alkylation of Thiols - A one-dram vial with benzene (650 $\mu \mathrm{L}$, kept over $4 \AA$ molecular sieves) was sparged with $\mathrm{N}_{2}$ for 5 minutes. Alkene (equivalents specified for each substrate) and triethyl phosphite (1.5 equiv, $0.25 \mathrm{mmol}$ ) were added under positive $\mathrm{N}_{2}$ pressure. The reaction mixture was heated to $70{ }^{\circ} \mathrm{C}$ and a $0.16 \mathrm{M}$ solution of thiol (1 equiv, $0.16 \mathrm{~mol}$ ) and AIBN ( 0.15 equiv, $4.1 \mathrm{mg}$, or 0.25 equiv, $6.8 \mathrm{mg}$ ) in degassed benzene was added via syringe pump at a rate of $2.3 \mu \mathrm{L} / \mathrm{min}$. After the addition was completed, the reaction was let run an additional 4 hours. When no starting material was observed by TLC (visualized with $p$-anisaldehyde stain), the reaction mixture was concentrated en vacuo and then subjected to a vacuum of 50 mTor to remove triethyl phosphorothioate. The crude residue was purified via flash chromatography to afford the desired product.

\section{F. Characterization of Alkylation Products in Alkene Scope}

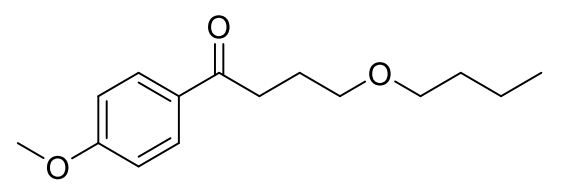

2a was synthesized via the general alkylation procedure above as a colorless oil ( $44 \mathrm{mg}, 93 \%$ yield).

Representative example of a reductive alkylation performed at $1 \mathrm{mmol}$ scale: A $25 \mathrm{~mL}$ roung-bottom flask containing n-butyl vinyl ether $(0.19 \mathrm{~mL}, 1.5 \mathrm{mmol}, 1.5$ equiv), triethyl phosphite $(0.26 \mathrm{~mL}, 1.5$ mmol, 1,5 equiv) and benzene ( $4 \mathrm{~mL}$ ) was sparged with nitrogen for 5 mins. The reaction mixture was heated to $70{ }^{\circ} \mathrm{C}$ while a solution of 1 (180 mg, $1.0 \mathrm{mmol}, 1$ equiv) and AIBN (25 mg, $0.15 \mathrm{mmol}, 0.15$ equiv) in degassed benzene $(6 \mathrm{~mL})$ was added over $7.5 \mathrm{~h}$. The reaction was let stir at $70{ }^{\circ} \mathrm{C}$ for an additional $12 \mathrm{~h}$ after which no starting material was observed by TLC (visualized with p-anisaldehyde stain), the reaction mixture was concentrated en vacuo and then subjected to a vacuum of $50 \mathrm{mTor}$ to remove triethyl phosphorothioate. The crude material was purified via flash column chromatography ( $15 \%$ ethyl acetate in hexanes) to afford $2 \mathbf{a}$ as a colorless oil ( $165 \mathrm{mg}, 86 \%$ yield).

Analytical data for $\mathbf{2 a}$ :

${ }^{1} \mathrm{H}-\mathrm{NMR}\left(300 \mathrm{MHz} ; \mathrm{CDCl}_{3}\right): \delta 7.92(\mathrm{~d}, \mathrm{~J}=9.0 \mathrm{~Hz}, 2 \mathrm{H}), 6.89$ (d, J = 8.9 Hz, 2H), $3.82(\mathrm{~s}, 3 \mathrm{H}), 3.45$ (t, J $=6.1 \mathrm{~Hz}, 2 \mathrm{H}$ ), $3.36(\mathrm{t}, \mathrm{J}=6.5 \mathrm{~Hz}, 2 \mathrm{H}$ ), $2.98(\mathrm{t}, \mathrm{J}=7.2 \mathrm{~Hz}, 2 \mathrm{H}$ ), 1.97 (quint, $\mathrm{J}=6.9,2 \mathrm{H}$ ), 1.51 (quint, $\mathrm{J}=$ 14.8, 6.8 Hz, 2H), $1.380-1.24(\mathrm{~m}, 2 \mathrm{H}), 0.87(\mathrm{t}, \mathrm{J}=7.3 \mathrm{~Hz}, 2 \mathrm{H})$

${ }^{13} \mathrm{C}-\mathrm{NMR}\left(126 \mathrm{MHz} ; \mathrm{CDCl}_{3}\right): \delta$ 198.94, 163.44, 130.46, 130.20, 113.80, 70.80, 69.98, 55.67, 34.95, $31.95,24.60,19.52,14.14$.

LRMS (APCI) Calc. for $\left[\mathrm{C}_{15} \mathrm{H}_{22} \mathrm{O}_{3}+\mathrm{Na}\right]^{+}=273.15$, Found $=273.13$

HRMS (ESI) Calc. for $\left[\mathrm{C}_{15} \mathrm{H}_{22} \mathrm{O}_{3}\right]^{+}=251.1642$, Found $=251.1639$

$\mathbf{R}_{\mathbf{f}}=0.35$ in $15 \%$ ethyl acetate/hexanes 


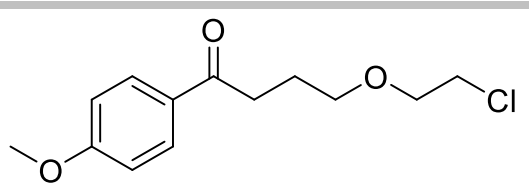

3 was synthesized via the general alkylation procedure above as a yellow oil ( $23 \mathrm{mg}, 60 \%$ yield).

Analytical data for 3:

${ }^{1} \mathrm{H}-\mathrm{NMR}\left(300 \mathrm{MHz} ; \mathrm{CDCl}_{3}\right)$ : $\delta 7.93(\mathrm{~d}, \mathrm{~J}=8.93 \mathrm{~Hz}, 2 \mathrm{H}), 6.90(\mathrm{~d}, \mathrm{~J}=8.93 \mathrm{~Hz}, 2 \mathrm{H}), 3.83(\mathrm{~s}, 3 \mathrm{H}), 3.70$ $3.62(\mathrm{~m}, 2 \mathrm{H}), 3.61-3.51(\mathrm{~m}, 4 \mathrm{H}), 3.02(\mathrm{t}, \mathrm{J}=7.1 \mathrm{~Hz}, 2 \mathrm{H}), 2.00$ (quint, $\mathrm{J}=7.1,6.2 \mathrm{~Hz}, 2 \mathrm{H}$ )

${ }^{13} \mathrm{C}-N M R\left(126 \mathrm{MHz} ; \mathrm{CDCl}_{3}\right): \delta$ 199.14, 163.45, 130.48, 130.23, 113.79, 76.05, 56.23, 55.65, 34.01, $30.87,19.23$

LRMS (APCl) Calc. for $\left[\mathrm{C}_{13} \mathrm{H}_{18} \mathrm{ClO}_{3}+\mathrm{H}\right]^{+}=257.09$, Found $=257.09$

HRMS (ESI) Calc. for $\left[\mathrm{C}_{13} \mathrm{H}_{17} \mathrm{ClO}_{3}\right]^{+}=257.0939$, Found $=257.0937$

$\mathbf{R}_{\mathbf{f}}=0.30$ in $20 \%$ ethyl acetate/hexanes

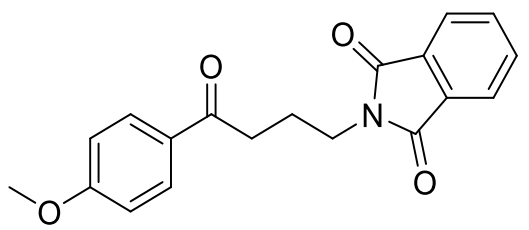

4 was synthesized via the general alkylation procedure as a white solid ( $19 \mathrm{mg}, 36 \%$ yield).

Analytical data for $\mathbf{4}$ :

${ }^{1} \mathrm{H}-\mathrm{NMR}\left(300 \mathrm{MHz} ; \mathrm{CDCl}_{3}\right): \delta 7.87$ (d, J = 8.9 Hz, 2H), 7.80 (dd, J = 5.4, 3.1 Hz, 2H), 7.67 (dd, J = 5.4, $3.1 \mathrm{~Hz}, 2 \mathrm{H}), 6.87(\mathrm{~d}, \mathrm{~J}=8.9 \mathrm{~Hz}, 2 \mathrm{H}), 3.82(\mathrm{~s}, 3 \mathrm{H}), 3.78(\mathrm{t}, \mathrm{J}=6.8 \mathrm{~Hz}, 2 \mathrm{H}), 2.97(\mathrm{t}, \mathrm{J}=7.3 \mathrm{~Hz}, 2 \mathrm{H}), 2.09$ (quint, $\mathrm{J}=7.36 \mathrm{~Hz}, 2 \mathrm{H}$ ).

${ }^{13}$ C-NMR (126 MHz; $\left.\mathrm{CDCl}_{3}\right): \delta$ 198.48, 163.59, 156.21, 130.43, 130.04, 113.84, 55.59, 40.33, 35.53, $28.52,24.80$.

LRMS (APCl) Calc. for $\left[\mathrm{C}_{19} \mathrm{H}_{17} \mathrm{NO}_{4}+\mathrm{H}\right]^{+}=324.12$, Found $=324.11$

HRMS (ESI) Calc. for $\left[\mathrm{C}_{19} \mathrm{H}_{17} \mathrm{NO}_{4}+\mathrm{H}\right]^{+}=324.1230$, Found $=324.1226$.

$\mathbf{R}_{\mathbf{f}}=0.1$ in $15 \%$ ethyl acetate/hexanes<smiles>COc1ccc(C(=O)CCCNC(=O)OC(C)(C)C)cc1</smiles>

5 was synthesized via the general alkylation procedure above a tan solid ( $38 \mathrm{mg}, 79 \%$ yield).

Analytical data for $\mathbf{5}$ :

${ }^{1} \mathrm{H}-\mathrm{NMR}\left(300 \mathrm{MHz} ; \mathrm{CDCl}_{3}\right): \delta 7.89(\mathrm{~d}, \mathrm{~J}=8.97 \mathrm{~Hz}, 2 \mathrm{H}), 6.88(\mathrm{~d}, \mathrm{~J}=8.87 \mathrm{~Hz}, 2 \mathrm{H}), 4.69(\mathrm{~s}, 1 \mathrm{H}), 3.82(\mathrm{~s}$, $3 \mathrm{H}), 3.22-3.02(\mathrm{~m}, 2 \mathrm{H}), 2.93(\mathrm{t}, \mathrm{J}=7.2 \mathrm{~Hz}, 2 \mathrm{H}), 1.87$ (dd, J = 13.8, $6.7 \mathrm{~Hz}, 2 \mathrm{H}), 1.39(\mathrm{~s}, 9 \mathrm{H})$. 
${ }^{13} \mathrm{C}-\mathrm{NMR}\left(126 \mathrm{MHz} ; \mathrm{CDCl}_{3}\right): \delta$ 198.55, 163.59, 156.22, 130.47, 130.01, 113.86, 113.85, 79.30, 55.65, 40.34, 35.56, 28.55, 24.78.

LRMS (APCI) Calc. for $\left[\mathrm{C}_{16} \mathrm{H}_{23} \mathrm{NO}_{4}+\mathrm{Na}\right]^{+}=316.16$, Found $=316.14$

HRMS (APCI) Calc. for $\left[\mathrm{C}_{16} \mathrm{H}_{23} \mathrm{NO}_{4}+\mathrm{Na}\right]^{+}=316.1519$, Found $=316.1524$

$\mathbf{R}_{\mathbf{f}}=0.1$ in $15 \%$ ethyl acetate/hexanes

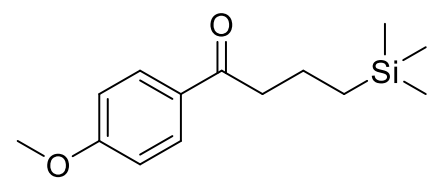

6 was synthesized via the general alkylation procedure above as a yellow oil ( $22 \mathrm{mg}, 54 \%$ yield).

Analytical data for 6 :

${ }^{1} \mathrm{H}-\mathrm{NMR}\left(300 \mathrm{MHz} ; \mathrm{CDCl}_{3}\right): \delta 7.94(\mathrm{~d}, \mathrm{~J}=9.0 \mathrm{~Hz}, 2 \mathrm{H}), 6.93(\mathrm{~d}, \mathrm{~J}=9.0 \mathrm{~Hz}, 2 \mathrm{H}), 3.86(\mathrm{~s}, 3 \mathrm{H}), 2.93(\mathrm{t}, \mathrm{J}$ $=7.3,2 \mathrm{H}), 1.72(\mathrm{~m}, 2 \mathrm{H}), 0.57(\mathrm{~m}, 2 \mathrm{H}), 0.01(\mathrm{~s}, 9 \mathrm{H})$

${ }^{13}$ C-NMR (126 MHz; $\left.\mathrm{CDCl}_{3}\right): \delta$ 199.5, 163.4, 130.5, 130.3, 113.9, 55.6, 42.2, 19.5, 16.9, 1.5

LRMS (APCI) Calc. for $\left[\mathrm{C}_{14} \mathrm{H}_{22} \mathrm{O}_{2} \mathrm{Si}\right]^{+}=250.14$, Found $=250.13$

HRMS (ESI) Calc. for $\left[\mathrm{C}_{14} \mathrm{H}_{22} \mathrm{O}_{2} \mathrm{Si}+\mathrm{H}\right]^{+}=251.1462$, Found $=251.1457$

$\mathbf{R}_{\mathbf{f}}=0.3$ in $7.5 \%$ EtOAc/Hexanes

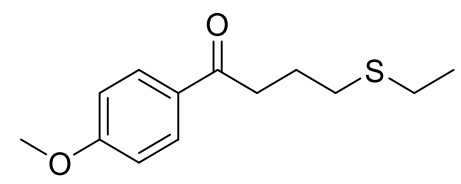

7 was synthesized via the general alkylation procedure as a yellow oil ( $23 \mathrm{mg}, 59 \%$ yield).

Analytical data for 7 :

${ }^{1} \mathrm{H}-N M R\left(300 \mathrm{MHz} ; \mathrm{CDCl}_{3}\right): \delta 8.01-7.82(\mathrm{~m}, 2 \mathrm{H}), 6.96-6.85(\mathrm{~m}, 2 \mathrm{H}), 3.83(\mathrm{~s}, 3), 3.03(\mathrm{t}, \mathrm{J}=7.2 \mathrm{~Hz}$, 2H), 2.60 (t, J = 7.0 Hz, 2H), 2.51 (q, J = 7.4 Hz, 2H), 1.99 (quin, J = 7.1 Hz, 2H), 1.22 (t, J = 7.4 Hz, $3 \mathrm{H})$.

${ }^{13}$ C-NMR (126 MHz; $\left.\mathrm{CDCl}_{3}\right): \delta$ 198.46, 163.55, 130.47, 130.12, 113.97, 113.84, 55.65, 36.95, 31.26, 25.82, 23.95, 14.93.

LRMS (APCI) Calc. for $\left[\mathrm{C}_{13} \mathrm{H}_{18} \mathrm{O}_{2} \mathrm{~S}\right]^{+}=238.10$, Found $=238.07$

HRMS (ESI) Calc. for $\left[\mathrm{C}_{13} \mathrm{H}_{18} \mathrm{O}_{2} \mathrm{~S}+\mathrm{H}\right]^{+}=239.1100$, Found $=239.1110$

$\mathbf{R}_{\mathbf{f}}=0.6$ in $15 \%$ ethyl acetate/hexanes 


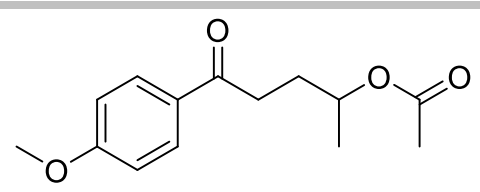

8 was synthesized via the general alkylation procedure as a colorless oil ( $31 \mathrm{mg}, 75 \%$ yield).

Analytical data for 8:

${ }^{1} \mathrm{H}-\mathrm{NMR}\left(300 \mathrm{MHz} ; \mathrm{CDCl}_{3}\right): \delta 7.89(\mathrm{~d}, \mathrm{~J}=8.89 \mathrm{~Hz}, 2 \mathrm{H}), 6.89(\mathrm{~d}, \mathrm{~J}=8.83 \mathrm{~Hz}, 2 \mathrm{H}), 4.95$ (quint, J = 6.25 $\mathrm{Hz}, 1 \mathrm{H}), 3.83(\mathrm{~s}, 3 \mathrm{H}), 2.91-3.05(\mathrm{~m}, 2 \mathrm{H}), 1.91-1.99(\mathrm{~m}, 2 \mathrm{H}), 1.23(\mathrm{~d}, \mathrm{~J}=6.3 \mathrm{~Hz}, 3 \mathrm{H})$

${ }^{13}$ C-NMR (126 MHz; $\left.\mathrm{CDCl}_{3}\right): \delta$ 198.05, 170.98, 163.54, 130.40, 129.94, 113.79, 70.74, 55.66, 34.27, $30.45,21.52,20.31$

HRMS (APCI) Calc. for $\left[\mathrm{C}_{14} \mathrm{H}_{18} \mathrm{O}_{4}+\mathrm{Na}\right]^{+}=273.1103$, Found $=273.1100$

$\mathbf{R}_{\mathbf{f}}=0.3$ in $15 \%$ EtOAc/Hexanes<smiles>COc1ccc(C(=O)CCC(C)OC)cc1</smiles>

9 was synthesized via the general alkylation procedure above as a yellow oil ( $28 \mathrm{mg}, 77 \%$ yield).

Analytical data for 9:

${ }^{1} \mathrm{H}-\mathrm{NMR}\left(300 \mathrm{MHz} ; \mathrm{CDCl}_{3}\right): \delta 7.92(\mathrm{~d}, \mathrm{~J}=9.0 \mathrm{~Hz}, 1 \mathrm{H}), 6.89(\mathrm{~d}, \mathrm{~J}=9.0 \mathrm{~Hz}, 1 \mathrm{H}), 3.82(\mathrm{~s}, 2 \mathrm{H}), 3.36$ (quint, $\mathrm{J}=7.3,1 \mathrm{H}), 3.27(\mathrm{~s}, 3 \mathrm{H}), 2.95-3.02(\mathrm{~m}, 2 \mathrm{H}), 2.00-1.73(\mathrm{~m}, 2 \mathrm{H}), 1.14(\mathrm{~d}, \mathrm{~J}=6.1 \mathrm{~Hz}, 3 \mathrm{H})$.

${ }^{13}$ C-NMR (126 MHz; $\left.\mathrm{CDCl}_{3}\right): \delta$ 198.98, 163.30, 130.62, 130.32, 130.06, 113.64, 75.91, 56.08, 33.86, $30.73,19.08$

LRMS (APCI) Calc. for $\left[\mathrm{C}_{13} \mathrm{H}_{18} \mathrm{O}_{3}+\mathrm{H}\right]^{+}=223.13$, Found $=223.11$

HRMS (ESI) Calc. for $\left[\mathrm{C}_{13} \mathrm{H}_{18} \mathrm{O}_{3}+\mathrm{Na}\right]^{+}=245.1148$, Found $=245.1148$

$\mathbf{R}_{\mathbf{f}}=0.3$ in $15 \%$ ethyl acetate/hexanes

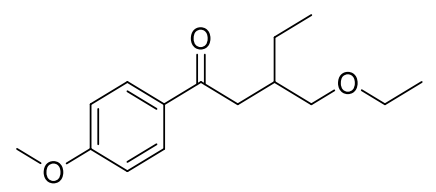

10 was synthesized via the general alkylation procedure above as a colorless oil ( $31 \mathrm{mg}, 75 \%$ yield). Analytical data for 10: 
${ }^{1} \mathrm{H}-\mathrm{NMR}\left(300 \mathrm{MHz} ; \mathrm{CDCl}_{3}\right): \delta 7.93(\mathrm{~d}, \mathrm{~J}=8.9 \mathrm{~Hz}, 2 \mathrm{H}), 6.89(\mathrm{~d}, \mathrm{~J}=8.9 \mathrm{~Hz}, 2 \mathrm{H}), 3.83(\mathrm{~s}, 3 \mathrm{H}), 3.43-$ $3.19(\mathrm{~m}, 4 \mathrm{H}), 3.03(\mathrm{dd}, \mathrm{J}=15.9,6.7 \mathrm{~Hz}, 1 \mathrm{H}), 2.74(\mathrm{dd}, \mathrm{J}=15.9,6.5 \mathrm{~Hz}, 1 \mathrm{H}), 2.24(\mathrm{dt}, \mathrm{J}=11.8,6.5 \mathrm{~Hz}$, $1 \mathrm{H}), 1.49-1.28(\mathrm{~m}, 2 \mathrm{H}), 1.10(\mathrm{t}, \mathrm{J}=7.0 \mathrm{~Hz}, 3 \mathrm{H}), 0.89(\mathrm{t}, \mathrm{J}=7.5 \mathrm{~Hz}, 3 \mathrm{H})$.

${ }^{13}$ C-NMR (126 MHz; $\left.\mathrm{CDCl}_{3}\right): \delta$ 199.23, 163.36, 130.66, 130.57, 113.71, 73.33, 66.42, 55.62, 40.38, $37.03,24.63,15.31,11.65$.

LRMS (APCI) Calc. for $\left[\mathrm{C}_{15} \mathrm{H}_{22} \mathrm{O}_{3}\right]^{+}=250.16$, Found $=250.11$

HRMS (ESI) Calc. for $\left[\mathrm{C}_{15} \mathrm{H}_{22} \mathrm{O}_{3}+\mathrm{H}\right]^{+}=251.1642$, Found $=251.1639$

$\mathbf{R}_{\mathbf{f}}=0.3$ in $20 \%$ ethyl acetate/hexanes

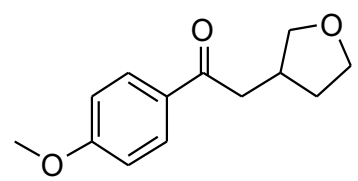

11 was synthesized via the general alkylation procedure as white needles ( $35 \mathrm{mg}, 72 \%$ yield).

Analytical data for 11:

${ }^{1} \mathrm{H}-N M R\left(300 \mathrm{MHz} ; \mathrm{CDCl}_{3}\right): \delta 7.95(\mathrm{~d}, \mathrm{~J}=8.27 \mathrm{~Hz}, 2 \mathrm{H}), 6.95(\mathrm{~d}, \mathrm{~J}=8.92,2 \mathrm{H}), 4.05$ (dd, J = 8.5, 7.1 $\mathrm{Hz}, 1 \mathrm{H}), 3.95-3.86(\mathrm{~m}, 4 \mathrm{H}), 3.80(\mathrm{dd}, \mathrm{J}=15.8,7.5 \mathrm{~Hz}, 2 \mathrm{H}), 3.46(\mathrm{dd}, \mathrm{J}=8.6,6.3 \mathrm{~Hz}, 1 \mathrm{H}), 3.18-2.98$ $(\mathrm{m}, 2 \mathrm{H}), 2.83(\mathrm{dp}, \mathrm{J}=14.1,7.1 \mathrm{~Hz}, 1 \mathrm{H}), 2.21(\mathrm{dtd}, \mathrm{J}=12.6,7.7,5.1 \mathrm{~Hz}, 1 \mathrm{H}), 1.61$ (ddd, J = 14.8, 12.4, $7.4 \mathrm{~Hz}, 1 \mathrm{H})$.

${ }^{13} \mathrm{C}-\mathrm{NMR}\left(126 \mathrm{MHz} ; \mathrm{CDCl}_{3}\right): \delta$ 197.80, 163.63, 130.42, 129.98, 113.91, 73.46, 67.86, 55.70, 42.20, $34.87,32.41$.

LRMS (APCI) Calc. for $\left[\mathrm{C}_{13} \mathrm{H}_{16} \mathrm{O}_{3}+\mathrm{H}\right]^{+}=221.12$, Found $=221.12$

HRMS (ESI) Calc. for $\left[\mathrm{C}_{13} \mathrm{H}_{16} \mathrm{O}_{3}+\mathrm{H}\right]^{+}=221.1172$, Found $=221.1172$

$\mathbf{R}_{\mathbf{f}}=0.1$ in $15 \%$ ethyl acetate/hexanes

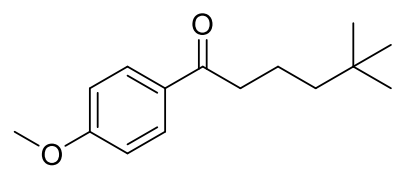

12 was synthesized via the general alkylation procedure above as a white, amorphous solid (32 mg, $83 \%$ yield).

Analytical data for 12:

${ }^{1} \mathrm{H}-\mathrm{NMR}\left(300 \mathrm{MHz} ; \mathrm{CDCl}_{3}\right): \delta 7.94$ (d, J = 9.0 Hz, 2H), 6.93 (d, J = 9.0 Hz, 2H), 1.96 (s, 3H), 2.89 (t, J $=7.0 \mathrm{~Hz}, 2 \mathrm{H}), 1.69(\mathrm{~m}, 2 \mathrm{H}), 1.25(\mathrm{~m}, 2 \mathrm{H}), 0.9(\mathrm{~s}, 3 \mathrm{H})$,

${ }^{13}$ C-NMR (126 MHz; $\left.\mathrm{CDCl}_{3}\right): \delta$ 199.4, 163.4, 130.4, 130.3, 113.9, 55.7, 44.0, 39.2, 30.6, 29.5, 20.0

LRMS (APCI) Calc. for $\left[\mathrm{C}_{15} \mathrm{H}_{22} \mathrm{O}_{2}\right]^{+}=234.16$, Found $=234.16$ 
HRMS (ESI) Calc. for $\left[\mathrm{C}_{15} \mathrm{H}_{22} \mathrm{O}_{2}+\mathrm{H}\right]^{+}=235.1693$, Found $=235.1695$

$\mathbf{R}_{\mathbf{f}}=0.3$ in $15 \%$ ethyl acetate/hexanes<smiles>COc1ccc(C(=O)CCCCc2ccccc2)cc1</smiles>

13 was synthesized via the general alkylation procedure above as a white solid ( $32 \mathrm{mg}, 72 \%$ yield).

Analytical data for 13:

${ }^{1} \mathrm{H}-\mathrm{NMR}\left(300 \mathrm{MHz} ; \mathrm{CDCl}_{3}\right): \delta 7.96(\mathrm{~d}, \mathrm{~J}=8.9 \mathrm{~Hz}, 2 \mathrm{H}), 7.30$ (t, J = 9.2 Hz, 2H), 7.22 (d, J = 7.2 Hz, $3 \mathrm{H}), 6.95(\mathrm{~d}, \mathrm{~J}=8.9 \mathrm{~Hz}, 2 \mathrm{H}), 3.89(\mathrm{~s}, 3 \mathrm{H}), 2.96(\mathrm{t}, \mathrm{J}=7.1 \mathrm{~Hz}, 2 \mathrm{H}), 2.70(\mathrm{t}, \mathrm{J}=7.3 \mathrm{~Hz}, 2 \mathrm{H}), 1.68-1.86$ $(\mathrm{m}, 4 \mathrm{H})$

${ }^{13} \mathrm{C}-\mathrm{NMR}\left(126 \mathrm{MHz} ; \mathrm{CDCl}_{3}\right): \delta 199.10,163.45,142.44,130.45,130.21,128.55,128.44,125.86$, $113.80,55.61,38.22,36.98,31.35,24.37$

LRMS (APCI) Calc. for $\left[\mathrm{C}_{18} \mathrm{H}_{20} \mathrm{O}_{2}\right]^{+}=268.15$, Found $=268.14$

HRMS (ESI) Calc. for $\left[\mathrm{C}_{18} \mathrm{H}_{20} \mathrm{O}_{2}+\mathrm{H}\right]^{+}=269.1536$, Found $=269.1533$

$\mathbf{R}_{\mathbf{f}}=0.6$ in $15 \%$ ethyl acetate/hexanes

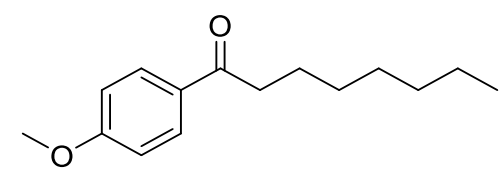

14 was synthesized via the general alkylation procedure as a colorless oil ( $16 \mathrm{mg}, 44 \%$ yield).

Analytical data for 14 :

${ }^{1} \mathrm{H}-\mathrm{NMR}\left(300 \mathrm{MHz} ; \mathrm{CDCl}_{3}\right): \delta 7.94(\mathrm{~d}, \mathrm{~J}=9.0 \mathrm{~Hz}, 2 \mathrm{H}), 6.92(\mathrm{~d}, \mathrm{~J}=9.0 \mathrm{~Hz}, 2 \mathrm{H}), 3.86(\mathrm{~s}, 3 \mathrm{H}), 2.89(\mathrm{t}, \mathrm{J}=$ $7.0,2 \mathrm{H}), 1.72$ (quint, $2 \mathrm{H}), 1.23-1.49(\mathrm{~m}, 8 \mathrm{H}), 3.82(\mathrm{t}, 3 \mathrm{H})$

${ }^{13} \mathrm{C}-\mathrm{NMR}\left(126 \mathrm{MHz} ; \mathrm{CDCl}_{3}\right): \delta$ 199.5, 163.4, 130.5, 130.3, 113.7, 55.7, 38.5, 31.9, 29.6, 24.8, 22.8, 14.3

HRMS (APCl) Calc. for $\left[\mathrm{C}_{15} \mathrm{H}_{22} \mathrm{O}_{2}+\mathrm{H}\right]^{+}=235.1698$, Found $=235.1693$

$\mathbf{R}_{\mathbf{f}}=0.5$ in $15 \%$ ethyl acetate/hexanes<smiles>COc1ccc(C(=O)CCCCCO)cc1</smiles>

15 was synthesized via the general alkylation procedure above as a white solid that reddened on standing (24 mg, 66\% yield).

Analytical data for 15 : 
${ }^{1} \mathrm{H}-\mathrm{NMR}\left(300 \mathrm{MHz} ; \mathrm{CDCl}_{3}\right): \delta 7.29(\mathrm{~d}, \mathrm{~J}=9.0 \mathrm{~Hz}, 2 \mathrm{H}), 6.91$ (d, J = 9.0 Hz, 2H), $3.84(\mathrm{~s}, 3), 3.65$ (t, J = $7.2 \mathrm{~Hz}, 2 \mathrm{H}$ ), $2.92(\mathrm{t}, \mathrm{J}=7.4 \mathrm{~Hz}, 2 \mathrm{H}$ ), 1.76 (quint, $\mathrm{J}=7.2 \mathrm{~Hz}, 2 \mathrm{H}), 1.60(\mathrm{~d}, \mathrm{~J}=9.0 \mathrm{~Hz}, 2 \mathrm{H}$ ), $1.45(\mathrm{~d}, \mathrm{~J}=$ $9.0 \mathrm{~Hz}, 2 \mathrm{H})$,

${ }^{13}$ C-NMR (126 MHz; $\left.\mathrm{CDCl}_{3}\right): \delta$ 199.3, 163.5, 130.4, 130.2, 113.8, 62.8, 56.6, 38.2, 32.6, 25.6, 24.2

LRMS (APCI) Calc. for $\left[\mathrm{C}_{13} \mathrm{H}_{18} \mathrm{O}_{3}+\mathrm{H}\right]^{+}=223.13$, Found $=223.15$

HRMS (ESI) Calc. for $\left[\mathrm{C}_{13} \mathrm{H}_{18} \mathrm{O}_{3}+\mathrm{H}\right]^{+}=223.1329$, Found $=223.1329$

$\mathbf{R}_{\mathbf{f}}=0.3$ in $15 \%$ ethyl acetate/hexanes

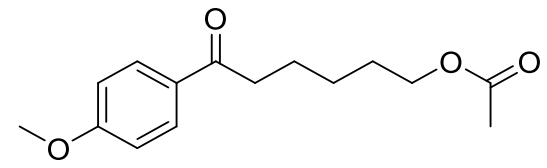

16 was synthesized via the general alkylation procedure as a yellow oil ( $30 \mathrm{mg}, 69 \%$ yield).

Analytical data for 16:

${ }^{1} \mathrm{H}-\mathrm{NMR}\left(300 \mathrm{MHz} ; \mathrm{CDCl}_{3}\right): \delta 7.90(\mathrm{~d}, \mathrm{~J}=8.97 \mathrm{~Hz}, 2 \mathrm{H}), 6.89(\mathrm{~d}, \mathrm{~J}=8.97 \mathrm{~Hz}, 2 \mathrm{H}), 4.03$ (t, J = $6.7 \mathrm{~Hz}$, $2 \mathrm{H}), 3.83(\mathrm{~s}, 3 \mathrm{H}), 2.89(\mathrm{t}, \mathrm{J}=7.3 \mathrm{~Hz}, 2 \mathrm{H}), 2.00(\mathrm{~s}, 3 \mathrm{H}), 1.81-1.57(\mathrm{~m}, 4 \mathrm{H}), 1.42$ (ddd, J = 12.4, 7.3, $2.2 \mathrm{~Hz}, 2 \mathrm{H})$.

${ }^{13} \mathrm{C}-\mathrm{NMR}\left(126 \mathrm{MHz} ; \mathrm{CDCl}_{3}\right): \delta$ 198.90, 171.41, 163.46, 130.22, 113.85, 113.80, 64.54, 55.61, 38.16, 28.66, 25.89, 24.22, 21.19.

LRMS (APCI) Calc. for $\left[\mathrm{C}_{15} \mathrm{H}_{20} \mathrm{O}_{4}+\mathrm{H}\right]^{+}=264.14$, Found $=265.13$

HRMS (ESI) Calc. for $\left[\mathrm{C}_{15} \mathrm{H}_{20} \mathrm{O}_{4}+\mathrm{Na}\right]^{+}=287.1254$, Found $=287.1254$

$\mathbf{R}_{\mathbf{f}}=0.3$ in $15 \%$ ethyl acetate/hexanes

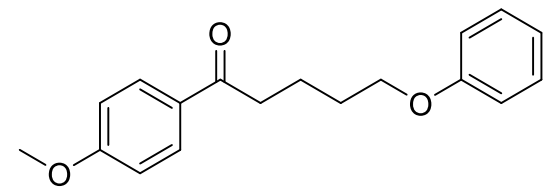

17 was synthesized via the general alkylation procedure above as a white solid ( $35 \mathrm{mg}, 75 \%$ yield).

Analytical data for 17:

${ }^{1} \mathrm{H}-\mathrm{NMR}\left(300 \mathrm{MHz} ; \mathrm{CDCl}_{3}\right): \delta 7.85(\mathrm{~d}, \mathrm{~J}=9.0 \mathrm{~Hz}, 2 \mathrm{H}), 7.10-7.21(\mathrm{~m}, 3 \mathrm{H}), 6.77-6.86(\mathrm{~m}, 4 \mathrm{H}), 3.90(\mathrm{t}, \mathrm{J}$ $=6.0 \mathrm{~Hz}, 2 \mathrm{H}), 3.77(\mathrm{~s}, 3 \mathrm{H}), 2.91(\mathrm{t}, \mathrm{J}=7.0 \mathrm{~Hz}, 2 \mathrm{H}), 1.74-1.88(\mathrm{~m}, 4 \mathrm{H})$,

${ }^{13}$ C-NMR (126 MHz; $\left.\mathrm{CDCl}_{3}\right): \delta$ 198.82, 163.5, 159.1, 130.5, 130.2, 129.6, 120.7, 114.6, 113.9, 67.6, $55.6,37.9,29.0,21.3$

LRMS (APCI) Calc. for $\left[\mathrm{C}_{18} \mathrm{H}_{20} \mathrm{O}_{3}+\mathrm{H}\right]^{+}=285.15$, Found $=285.13$

HRMS (ESI) Calc. for $\left[\mathrm{C}_{18} \mathrm{H}_{20} \mathrm{O}_{3}+\mathrm{H}\right]^{+}=285.1485$, Found $=285.1485$ 
$\mathbf{R}_{\mathbf{f}}=0.7$ in $15 \%$ ethyl acetate/hexanes

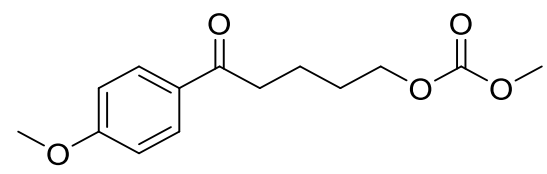

18 was synthesized via the general alkylation procedure above as a white solid that reddened on standing (29 $\mathrm{mg}, 66 \%$ yield).

Analytical data for 18:

${ }^{1} \mathrm{H}-\mathrm{NMR}\left(300 \mathrm{MHz} ; \mathrm{CDCl}_{3}\right): \delta 7.94(\mathrm{~d}, \mathrm{~J}=9.0 \mathrm{~Hz}, 2 \mathrm{H}), 6.94(\mathrm{~d}, \mathrm{~J}=9.0 \mathrm{~Hz}, 2 \mathrm{H}), 4.19(\mathrm{t}, \mathrm{J}=6.3 \mathrm{~Hz}, 2 \mathrm{H})$, $6.88(\mathrm{~s}, 3 \mathrm{H}), 3.79(\mathrm{~s}, 3 \mathrm{H}), 2.98(\mathrm{t}, \mathrm{J}=7.1 \mathrm{~Hz}, 2 \mathrm{H}), 1.74-1.90(\mathrm{~m}, 4 \mathrm{H})$

${ }^{13} \mathrm{C}$-NMR (126 MHz; $\left.\mathrm{CDCl}_{3}\right): \delta$ 198.4, 163.5, 156, 130.4, 130.0, 113.8, 68.0, 55.5, 54.8, 37.6, 28.4, 20.7

LRMS (APCI) Calc. for $\left[\mathrm{C}_{14} \mathrm{H}_{18} \mathrm{O}_{5}+\mathrm{Na}\right]^{+}=289.11$, Found $=289.14$

HRMS (ESI) Calc. for $\left[\mathrm{C}_{14} \mathrm{H}_{18} \mathrm{O}_{5}+\mathrm{H}\right]^{+}=267.1227$, Found $=267.1125$

$\mathbf{R}_{\mathbf{f}}=0.2$ in $15 \%$ ethyl acetate/hexanes<smiles>COc1ccc(C(=O)CCCCNC(=O)OC(C)(C)C)cc1</smiles>

19 was synthesized via the general alkylation procedure above as a tan, solid (51 $\mathrm{mg}, 75 \%$ yield).

Analytical data for 19:

${ }^{1} \mathrm{H}-\mathrm{NMR}\left(300 \mathrm{MHz} ; \mathrm{CDCl}_{3}\right): \delta 7.93(\mathrm{~d}, \mathrm{~J}=8.9 \mathrm{~Hz}, 2 \mathrm{H}), 6.92(\mathrm{~d}, \mathrm{~J}=8.9 \mathrm{~Hz}, 2 \mathrm{H}), 4.70(\mathrm{~s}, 1 \mathrm{H}), 3.86$ (s, $3 \mathrm{H}$ ), 3.15 (dd, J = 12.8, $6.4 \mathrm{~Hz}, 2 \mathrm{H}), 2.94(\mathrm{t}, \mathrm{J}=7.2 \mathrm{~Hz}, 2 \mathrm{H}), 1.84-1.66(\mathrm{~m}, 2 \mathrm{H}), 1.58$ (dd, J = 14.8, $7.2 \mathrm{~Hz}, 2 \mathrm{H}), 1.44(\mathrm{~s}, 9 \mathrm{H})$

${ }^{13}$ C-NMR (126 MHz; $\left.\mathrm{CDCl}_{3}\right): \delta 198.84,163.46,156.13,130.40,130.00,113.77,79.16,55.58,40.31$, $37.72,29.71,28.52,21.50$.

HRMS (APCI) Calc. for $\left[\mathrm{C}_{17} \mathrm{H}_{25} \mathrm{NO}_{4}+\mathrm{Na}\right]^{+}=330.1681$, Found $=330.1679$

$\mathbf{R}_{\mathbf{f}}=0.1$ in $15 \%$ ethyl acetate/hexanes<smiles>CCOC(=O)C(CCCCC(=O)c1ccc(OC)cc1)C(=O)OCC</smiles>

20 was synthesized via the general alkylation procedure above as a yellow oil (34 $\mathrm{mg}, 59 \%$ yield). Analytical data for $\mathbf{2 0}$ : 
${ }^{1} \mathrm{H}-\mathrm{NMR}\left(300 \mathrm{MHz} ; \mathrm{CDCl}_{3}\right): \delta 7.95$ (d, J = $\left.8.94 \mathrm{~Hz}, 2 \mathrm{H}\right), 6.94$ (d, J = $\left.8.94 \mathrm{~Hz}, 2 \mathrm{H}\right), 4.21$ (q, J = $7.1 \mathrm{~Hz}$, $4 \mathrm{H}), 3.89(\mathrm{~s}, 3 \mathrm{H}), 3.35(\mathrm{t}, \mathrm{J}=7.5 \mathrm{~Hz}, 2 \mathrm{H}), 2.94(\mathrm{t}, \mathrm{J}=7.4 \mathrm{~Hz}, 2 \mathrm{H}$ ), 1.98 (quint, $\mathrm{J}=7.31,2 \mathrm{H}$ ), 1.77 (quint, $\mathrm{J}=7.31,2 \mathrm{H}), 1.56-1.36(\mathrm{~m}, 2 \mathrm{H}), 1.28(\mathrm{t}, \mathrm{J}=7.1 \mathrm{~Hz}, 3 \mathrm{H})$.

${ }^{13}$ C-NMR (126 MHz; $\left.\mathrm{CDCl}_{3}\right): \delta 198.77,169.66,163.51,130.45,130.15,113.84,61.53,55.64,52.05$, $37.97,28.74,27.23,24.15,14.26$.

HRMS (APCI) Calc. for $\left[\mathrm{C}_{19} \mathrm{H}_{26} \mathrm{O}_{6}+\mathrm{H}\right]^{+}=351.1807$, Found $=351.1804$

$\mathbf{R}_{\mathbf{f}}=0.2$ in $15 \%$ ethyl acetate/hexanes

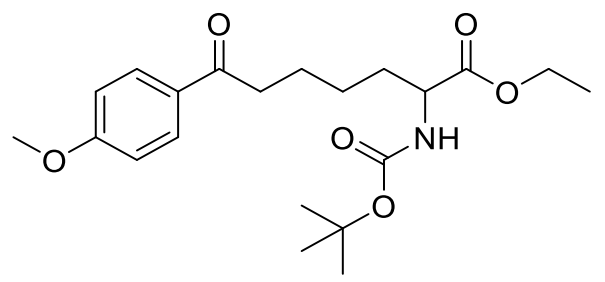

21 was synthesized via the general alkylation procedure as a colorless oil (13 mg, 20\% yield).

Analytical data for 21:

${ }^{1} \mathrm{H}-\mathrm{NMR}\left(300 \mathrm{MHz} ; \mathrm{CDCl}_{3}\right): \delta 7.90(\mathrm{~d}, \mathrm{~J}=9.0 \mathrm{~Hz}, 2 \mathrm{H}), 6.90(\mathrm{~d}, \mathrm{~J}=8.9 \mathrm{~Hz}, 2 \mathrm{H}), 4.99$ (d, J = 8.6 Hz, $1 \mathrm{H}), 4.10-4.30(\mathrm{~m}, 2 \mathrm{H}), 3.84(\mathrm{~s}, 3 \mathrm{H}), 2.93-2.79(\mathrm{~m}, 2 \mathrm{H}), 1.68(\mathrm{ddd}, \mathrm{J}=28.1,18.5,10.5 \mathrm{~Hz}, 6 \mathrm{H})$, $1.41(\mathrm{~s}, 9 \mathrm{H}), 1.24(\mathrm{t}, \mathrm{J}=7.1 \mathrm{~Hz}, 3 \mathrm{H})$.

${ }^{13}$ C-NMR (126 MHz; $\left.\mathrm{CDCl}_{3}\right): \delta$ 198.78, 173.04, 163.51, 155.56, 130.45, 130.12, 113.84, 79.99, 61.49, $55.65,53.55,38.09,32.92,28.49,25.27,24.20,14.38$.

LRMS (APCI) Calc. for $\left[\mathrm{C}_{21} \mathrm{H}_{31} \mathrm{NO}_{6}+\mathrm{Na}\right]^{+}=416.20$, Found $=416.22$

HRMS (ESI) Calc. for $\left[\mathrm{C}_{21} \mathrm{H}_{31} \mathrm{NO}_{6}+\mathrm{Na}\right]^{+}=416.2044$, Found $=416.2031$

$\mathbf{R}_{\mathbf{f}}=0.3$ in $35 \%$ ethyl acetate/hexanes

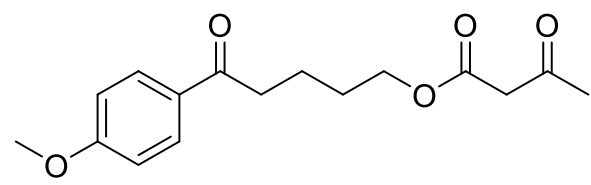

22 was synthesized via the general alkylation procedure above as a white, amorphous solid ( $23 \mathrm{mg}$, $48 \%$ yield).

Analytical data for 22:

${ }^{1} \mathrm{H}-\mathrm{NMR}\left(300 \mathrm{MHz} ; \mathrm{CDCl}_{3}\right): \delta 7.90(\mathrm{~d}, \mathrm{~J}=9.0 \mathrm{~Hz}, 1 \mathrm{H}), 6.90(\mathrm{~d}, \mathrm{~J}=9.0 \mathrm{~Hz}, 1 \mathrm{H}), 4.22(\mathrm{t}, \mathrm{J}=6.1 \mathrm{~Hz}, 1 \mathrm{H})$, $3.84(\mathrm{~s}, 2 \mathrm{H}), 3.42(\mathrm{~s}, 1 \mathrm{H}), 2.94$ (t, J = $6.8 \mathrm{~Hz}, 1 \mathrm{H}$ ), 1.77 (ddd, J = 6.1, 4.1, 1.6 Hz, 2H).

${ }^{13} \mathrm{C}-\mathrm{NMR}\left(126 \mathrm{MHz} ; \mathrm{CDCl}_{3}\right): \delta 200.83,198.41,167.31,163.53,130.39,130.03,113.83,65.28,55.61$, 50.19, 37.54, 29. 95, 28.19, 20.76. 
LRMS (APCI) Calc. for $\left[\mathrm{C}_{16} \mathrm{H}_{20} \mathrm{O}_{5}+\mathrm{Na}\right]^{+}=315.12$, Found $=315.16$

HRMS (APCI) Calc. for $\left[\mathrm{C}_{16} \mathrm{H}_{20} \mathrm{O}_{5}+\mathrm{Na}\right]^{+}=315.1203$, Found $=315.1199$

$\mathbf{R}_{\mathbf{f}}=0.3$ in $15 \%$ ethyl acetate/hexanes

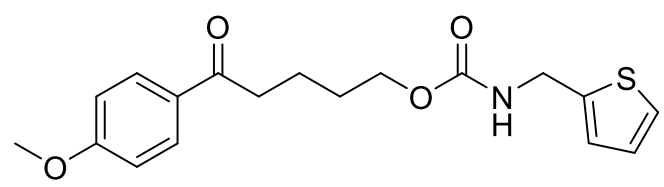

23 was synthesized via the general alkylation procedure above as a tan, solid ( $27 \mathrm{mg}, 47 \%$ yield).

Analytical data for 23:

${ }^{1} \mathrm{H}-\mathrm{NMR}\left(300 \mathrm{MHz} ; \mathrm{CDCl}_{3}\right): \delta 7.95(\mathrm{~d}, \mathrm{~J}=8.9 \mathrm{~Hz}, 2 \mathrm{H}), 7.25-7.14(\mathrm{~m}, 1 \mathrm{H}), 7.01-6.86(\mathrm{~m}, 2 \mathrm{H}), 5.06$ (s, 1H), $4.54(\mathrm{~d}, \mathrm{~J}=5.7 \mathrm{~Hz}, 2 \mathrm{H}), 4.26-4.05(\mathrm{~m}, 2 \mathrm{H}), 3.88(\mathrm{~s}, 3 \mathrm{H}), 2.97(\mathrm{t}, \mathrm{J}=7.1 \mathrm{~Hz}, 2 \mathrm{H}), 1.79$ (ddd, J = 23.6, $12.4,6.9 \mathrm{~Hz}, 4 \mathrm{H})$.

${ }^{13} \mathrm{C}$-NMR $\left(126 \mathrm{MHz} ; \mathrm{CDCl}_{3}\right): \delta 198.21,163.07,156.04,141.05,129.99,129.67,126.57,125.44$, $124.82,113.40,64.52,55.19,39.52,37.25,28.28,20.45$

LRMS (APCl) Calc. for $\left[\mathrm{C}_{18} \mathrm{H}_{21} \mathrm{NO}_{4} \mathrm{~S}+\mathrm{H}\right]^{+}=348.13$, Found $=348.16$

HRMS (ESI) Calc. for $\left[\mathrm{C}_{18} \mathrm{H}_{21} \mathrm{NO}_{4} \mathrm{~S}+\mathrm{H}\right]^{+}=348.1264$, Found $=348.1262$

$\mathbf{R}_{\mathbf{f}}=0.1$ in $15 \%$ ethyl acetate/hexanes<smiles>COc1ccc(C(=O)CCCCOC(=O)NCc2ccco2)cc1</smiles>

24 was synthesized via the general alkylation procedure above as a white solid that reddened on standing (17 $\mathrm{mg}, 31 \%$ yield).

Analytical data for 24 :

${ }^{1} \mathrm{H}-\mathrm{NMR}\left(300 \mathrm{MHz} ; \mathrm{CDCl}_{3}\right): \delta 7.96(2, \mathrm{~J}=8.91 \mathrm{~Hz}, 2 \mathrm{H}), 7.37(\mathrm{~s}, 1 \mathrm{H}), 6.95(\mathrm{~d}, \mathrm{~J}=8.91 \mathrm{~Hz}, 2 \mathrm{H}), 6.36-$ $6.30(\mathrm{~m}, 1 \mathrm{H}), 6.24(\mathrm{~s}, 1 \mathrm{H}), 4.99(\mathrm{~s}, 1 \mathrm{H}), 4.36(\mathrm{~d}, \mathrm{~J}=5.6 \mathrm{~Hz}, 2 \mathrm{H}), 4.15(\mathrm{t}, \mathrm{J}=6.1 \mathrm{~Hz}, 2 \mathrm{H}), 3.89(\mathrm{~s}, 3 \mathrm{H})$, $2.97(\mathrm{t}, \mathrm{J}=7.1 \mathrm{~Hz}, 2 \mathrm{H}), 1.91-1.65(\mathrm{~m}, 4 \mathrm{H})$

${ }^{13} \mathrm{C}-\mathrm{NMR}\left(126 \mathrm{MHz} ; \mathrm{CDCl}_{3}\right): \delta 198.70,163.51,156.57,151.71,142.36,130.45,130.08,113.84$, $110.54,107.38,64.96,55.65,38.14,37.70,28.71,20.88$

LRMS (APCI) Calc. for $\left[\mathrm{C}_{18} \mathrm{H}_{21} \mathrm{NO}_{5}+\mathrm{H}\right]^{+}=332.15$, Found $=332.17$

HRMS (ESI) Calc. for $\left[\mathrm{C}_{18} \mathrm{H}_{21} \mathrm{NO}_{5}+\mathrm{H}\right]^{+}=332.1492$, Found $=332.1489$

$\mathbf{R}_{\mathbf{f}}=0.1$ in $15 \%$ ethyl acetate/hexanes 


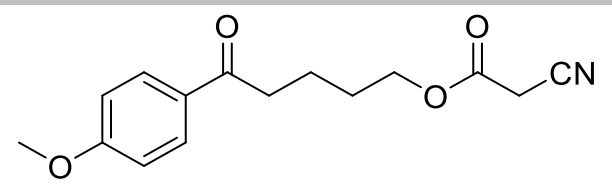

25 was synthesized via the general alkylation procedure above as a white, amorphous solid (22 mg, $49 \%$ yield).

Analytical data for 25:

${ }^{1} \mathrm{H}-\mathrm{NMR}\left(300 \mathrm{MHz} ; \mathrm{CDCl}_{3}\right): \delta 7.95(\mathrm{~d}, \mathrm{~J}=8.94 \mathrm{~Hz}, 2 \mathrm{H}), 6.94(\mathrm{~d}, \mathrm{~J}=8.94 \mathrm{~Hz}, 2 \mathrm{H}), 4.21(\mathrm{~m}, 2 \mathrm{H}), 3.89$ (s, 2H), $3.35(\mathrm{t}, \mathrm{J}=7.5 \mathrm{~Hz}, 1 \mathrm{H}), 2.94(\mathrm{t}, \mathrm{J}=7.4 \mathrm{~Hz}, 1 \mathrm{H}), 1.98(\mathrm{q}, \mathrm{J}=7.31,2 \mathrm{H}), 1.77$ (quint, J = 7.31, $2 \mathrm{H}), 1.56-1.36(\mathrm{~m}, 2 \mathrm{H}), 1.28(\mathrm{t}, \mathrm{J}=7.1 \mathrm{~Hz}, 3 \mathrm{H})$.

${ }^{13}$ C-NMR (126 MHz; $\left.\mathrm{CDCl}_{3}\right): \delta$ 198.25, 163.62, 163.13, 130.41, 129.99, 113.89, 113.21, 66.93, 55.66, $55.64,37.46,28.06,24.89,20.62$

LRMS (APCI) Calc. for $\left[\mathrm{C}_{15} \mathrm{H}_{17} \mathrm{NO}_{4}+\mathrm{H}\right]^{+}=276.12$, Found $=276.15$

HRMS (ESI) Calc. for $\left[\mathrm{C}_{15} \mathrm{H}_{17} \mathrm{NO}_{4}+\mathrm{Na}\right]^{+}=298.1050$, Found $=298.1053$

$\mathbf{R}_{\mathbf{f}}=0.2$ in $15 \%$ ethyl acetate/hexanes

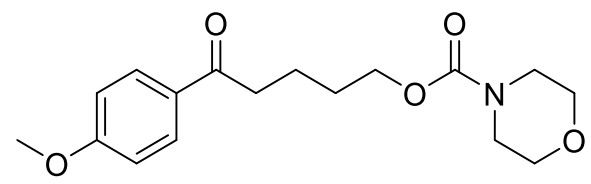

26 was synthesized via the general alkylation procedure above as a white, solid ( $37 \mathrm{mg}, 70 \%$ yield).

Analytical data for $\mathbf{2 6}$ :

${ }^{1} \mathrm{H}-\mathrm{NMR}\left(300 \mathrm{MHz} ; \mathrm{CDCl}_{3}\right): \delta 7.90(\mathrm{~d}, \mathrm{~J}=8.80 \mathrm{~Hz}, 2 \mathrm{H}), 6.90(\mathrm{~d}, \mathrm{~J}=9.0 \mathrm{~Hz}, 2 \mathrm{H}), 4.11$ (t, J = $6.1 \mathrm{~Hz}$, $2 \mathrm{H}), 3.84(\mathrm{~s}, 3 \mathrm{H}), 3.61(\mathrm{~s}, 4 \mathrm{H}), 3.53-3.33(\mathrm{~m}, 4 \mathrm{H}), 2.93(\mathrm{t}, \mathrm{J}=7.0 \mathrm{~Hz}, 2 \mathrm{H}), 1.86-1.59(\mathrm{~m}, 4 \mathrm{H})$.

${ }^{13}$ C-NMR (126 MHz; $\left.\mathrm{CDCl}_{3}\right): \delta 198.60,163.56,155.67,130.42,130.09,113.85,66.83,65.41,55.65$, $44.13,37.72,28.75,20.96$

LRMS (APCl) Calc. for $\left[\mathrm{C}_{17} \mathrm{H}_{23} \mathrm{NO}_{5}\right]^{+}=322.17$, Found $=322.12$

HRMS (ESI) Calc. for $\left[\mathrm{C}_{17} \mathrm{H}_{23} \mathrm{NO}_{5}+\mathrm{Na}\right]^{+}=344.1468$, Found $=344.1466$

$\mathbf{R}_{\mathbf{f}}=0.0$ in $15 \%$ ethyl acetate/hexanes<smiles>COc1ccc(C(=O)CCCCOCC2CO2)cc1</smiles>

27 was synthesized via the general alkylation procedure above as a white, solid that turned light pink on standing ( $21 \mathrm{mg}, 48 \%$ yield). 
Analytical data for 27:

${ }^{1} \mathrm{H}-\mathrm{NMR}\left(300 \mathrm{MHz} ; \mathrm{CDCl}_{3}\right.$ ): $\delta 7.91$ (d, J = 9.0 Hz, 2H), 6.89 (d, J = 9.0 Hz, 2H), 3.83 (s, 3H), 3.68 (dd, $\mathrm{J}=11.5,3.0 \mathrm{~Hz}, 1 \mathrm{H}), 3.59-3.42(\mathrm{~m}, 2 \mathrm{H}), 3.34(\mathrm{dd}, \mathrm{J}=11.5,5.8 \mathrm{~Hz}, 1 \mathrm{H}), 3.10(\mathrm{ddt}, \mathrm{J}=5.8,4.1,2.9$ $\mathrm{Hz}, 1 \mathrm{H}), 2.92(\mathrm{t}, \mathrm{J}=7.2 \mathrm{~Hz}, 2 \mathrm{H}), 2.75(\mathrm{dd}, \mathrm{J}=5.0,4.2 \mathrm{~Hz}, 1 \mathrm{H}), 2.57(\mathrm{dd}, \mathrm{J}=5.0,2.7 \mathrm{~Hz}, 1 \mathrm{H}), 1.86-$ $1.72(\mathrm{~m}, 2 \mathrm{H}), 1.72-1.57(\mathrm{~m}, 2 \mathrm{H})$

${ }^{13} \mathrm{C}-\mathrm{NMR}\left(126 \mathrm{MHz} ; \mathrm{CDCl}_{3}\right): \delta$ 198.97, 163.47, 130.46, 130.18, 113.81, 71.62, 71.44, 55.63, 51.04, $44.49,38.04,29.42,21.31$

LRMS (APCI) Calc. for $\left[\mathrm{C}_{15} \mathrm{H}_{20} \mathrm{O}_{4}+\mathrm{Na}\right]^{+}=287.13$, Found $=287.16$

HRMS (ESI) Calc. for $\left[\mathrm{C}_{15} \mathrm{H}_{20} \mathrm{O}_{4}\right]^{+}=265.1434$, Found $=265.1434$

$\mathbf{R}_{\mathbf{f}}=0.2$ in $15 \%$ ethyl acetate/hexanes<smiles>COc1ccc(C(=O)CCCCc2ccccc2OC(C)=O)cc1</smiles>

28 was synthesized via the general alkylation procedure above as a white, solid that turned orange on standing (43 $\mathrm{mg}, 80 \%$ yield).

Analytical data for 28:

${ }^{1} \mathrm{H}-N M R\left(300 \mathrm{MHz} ; \mathrm{CDCl}_{3}\right): \delta 7.97(\mathrm{~d}, \mathrm{~J}=9.0 \mathrm{~Hz}, 2 \mathrm{H}), 7.05-7.22(\mathrm{~m}, 2 \mathrm{H}), 6.95(\mathrm{~d}, \mathrm{~J}=9.0 \mathrm{~Hz}, 2 \mathrm{H})$, $6.64-74(\mathrm{~m}, 2 \mathrm{H}), 5.53(\mathrm{~s}, 3 \mathrm{H}), 3.89(\mathrm{~s}, 3 \mathrm{H}), 3.00(\mathrm{t}, \mathrm{J}=7.1 \mathrm{~Hz}, 2 \mathrm{H}), 2.75-2.64(\mathrm{~m}, 2 \mathrm{H}), 1.91-1.64$ $(\mathrm{m}, 4 \mathrm{H})$

${ }^{13}$ C-NMR (126 MHz; $\left.\mathrm{CDCl}_{3}\right): \delta 200.06,163.58,153.89,130.66,130.34,129.98,128.39,127.26$, $120.67,115.55,113.84,55.65,55.64,38.05,29.88,29.61,24.52$.

HRMS $(\mathrm{APCl})$ Calc. for $\left[\mathrm{C}_{18} \mathrm{H}_{20} \mathrm{O}_{3}+\mathrm{H}\right]^{+}=285.1491$, Found $=285.1486$

$\mathbf{R}_{\mathbf{f}}=0.1$ in $15 \%$ ethyl acetate/hexanes<smiles>COc1ccc(C(=O)CCCCc2ccccc2O)cc1</smiles>

29 was synthesized via the general alkylation procedure above as a yellow oil ( $27 \mathrm{mg}, 58 \%$ yield).

Analytical data for 29:

${ }^{1} \mathrm{H}-\mathrm{NMR}\left(300 \mathrm{MHz} ; \mathrm{CDCl}_{3}\right): \delta 7.95(\mathrm{~d}, \mathrm{~J}=9.0 \mathrm{~Hz}, 1 \mathrm{H}), 7.23-7.41(\mathrm{~m}, \mathrm{~J}=7.7,1.5 \mathrm{~Hz}, 3 \mathrm{H}), 7.03(\mathrm{dd}, \mathrm{J}$ = 7.6, $1.6 \mathrm{~Hz}, 1 \mathrm{H}), 6.97-6.89(\mathrm{~m}, 1 \mathrm{H}), 3.88(\mathrm{~s}, 3 \mathrm{H}), 2.95(\mathrm{t}, \mathrm{J}=7.1 \mathrm{~Hz}, 2 \mathrm{H}), 2.61(\mathrm{~d}, \mathrm{~J}=7.6 \mathrm{~Hz}, 2 \mathrm{H})$, $2.34(\mathrm{~s}, 3 \mathrm{H}), 1.91-1.60(\mathrm{~m}, 4 \mathrm{H})$. 
${ }^{13} \mathrm{C}-\mathrm{NMR}\left(126 \mathrm{MHz} ; \mathrm{CDCl}_{3}\right): \delta$ 198.91, 169.78, 163.46, 148.99, 134.10, 130.41, 130.32, 130.16, $127.15,126.26,122.40,113.81,55.61,38.09,30.22,29.74,24.42,21.10$

HRMS (APCI) Calc. for $\left[\mathrm{C}_{20} \mathrm{H}_{22} \mathrm{O}_{4}+\mathrm{H}\right]^{+}=327.1596$, Found $=327.1592$

$\mathbf{R}_{\mathbf{f}}=0.2$ in $15 \%$ ethyl acetate/hexanes

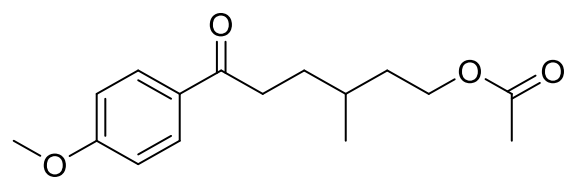

30 was synthesized via the general alkylation procedure as a yellow solid ( $35 \mathrm{mg}, 76 \%$ yield).

Analytical data for $\mathbf{3 0}$ :

${ }^{1} \mathrm{H}-\mathrm{NMR}\left(300 \mathrm{MHz} ; \mathrm{CDCl}_{3}\right): \delta 7.90(\mathrm{~d}, \mathrm{~J}=8.9 \mathrm{~Hz}, 2 \mathrm{H}), 6.89$ (d, J = 8.9 Hz, 2H), 4.07 (ddd, J = 11.0, 7.1, 3.8 Hz, 2H), $3.82(\mathrm{~s}, 3 \mathrm{H}), 2.98-2.76(\mathrm{~m}, 2 \mathrm{H}), 1.99(\mathrm{~s}, 3 \mathrm{H}), 1.84-1.39(\mathrm{~m}, 5 \mathrm{H}), 0.93(\mathrm{~d}, \mathrm{~J}=6.2$ $\mathrm{Hz}, 3 \mathrm{H})$.

${ }^{13} \mathrm{C}-N M R\left(126 \mathrm{MHz} ; \mathrm{CDCl}_{3}\right): \delta \quad 199.09,171.38,163.47,130.42,130.11,113.81,62.92,55.60,35.86$, $35.45,31.49,29.79,21.20,19.45$.

LRMS (APCI) Calc. for $\left[\mathrm{C}_{16} \mathrm{H}_{22} \mathrm{O}_{4}+\mathrm{H}\right]^{+}=279.16$, Found $=279.14$

HRMS (ESI) Calc. for $\left[\mathrm{C}_{16} \mathrm{H}_{22} \mathrm{O}_{4}+\mathrm{H}\right]^{+}=279.1591$, Found $=279.1591$

$\mathbf{R}_{\mathbf{f}}=0.3$ in $15 \%$ ethyl acetate/hexanes<smiles>COc1ccc(C(=O)CCC(C)CCO)cc1</smiles>

31 was synthesized via the general alkylation procedure above as a yellow oil ( $29 \mathrm{mg}, 75 \%$ yield).

Analytical data for 31:

${ }^{1} \mathrm{H}-N M R\left(300 \mathrm{MHz} ; \mathrm{CDCl}_{3}\right): \delta 7.90(\mathrm{~d}, \mathrm{~J}=9.26 \mathrm{~Hz}, 2 \mathrm{H}), 6.89(\mathrm{~d}, \mathrm{~J}=8.75 \mathrm{~Hz}, 2 \mathrm{H}), 3.88(\mathrm{~s}, 3 \mathrm{H}), 3.84-$ $3.60(\mathrm{~m}, 2 \mathrm{H}), 3.01-2.87(\mathrm{~m}, 2 \mathrm{H}), 1.92-1.78(\mathrm{~m}, 2 \mathrm{H}), 1.35-1.65(\mathrm{~m}, 3 \mathrm{H}), 0.98(\mathrm{~d}, \mathrm{~J}=6.4 \mathrm{~Hz}, 3 \mathrm{H})$.

${ }^{13}$ C-NMR (126 MHz; $\left.\mathrm{CDCl}_{3}\right): \delta$ 199.31, 163.28, 130.24, 129.90, 113.60, 60.74, 55.39, 39.49, 35.67, $30.90,29.10,19.63$

LRMS (APCI) Calc. for $\left[\mathrm{C}_{14} \mathrm{H}_{20} \mathrm{O}_{3}+\mathrm{H}\right]^{+}=237.15$, Found $=237.19$

HRMS (ESI) Calc. for $\left[\mathrm{C}_{14} \mathrm{H}_{20} \mathrm{O}_{3}+\mathrm{H}\right]^{+}=237.1485$, Found $=237.1486$

$\mathbf{R}_{\mathbf{f}}=0.0$ in $15 \%$ ethyl acetate/hexanes 


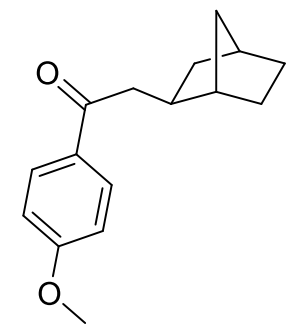

32 was synthesized via the general alkylation procedure above as a single diastereomer and off-white solid (27 mg, 67\% yield).

Analytical data for 32 :

${ }^{1} \mathrm{H}-\mathrm{NMR}\left(300 \mathrm{MHz} ; \mathrm{CDCl}_{3}\right): \delta 7.90$ (d, J = 7.6 Hz, 2H), 6.89 (d, J = 7.6 Hz, 2H), 3.83 (s, 3H), 2.78 (ddd, $\mathrm{J}=54.9,15.9,7.4 \mathrm{~Hz}, 2 \mathrm{H}), 2.19(\mathrm{~s}, 1 \mathrm{H}), 1.99(\mathrm{~d}, \mathrm{~J}=9.5 \mathrm{~Hz}, 2 \mathrm{H}), 1.68-0.97(\mathrm{~m}, 8 \mathrm{H})$.

${ }^{13} \mathrm{C}-N M R\left(126 \mathrm{MHz} ; \mathrm{CDCl}_{3}\right): \delta$ 198.97, 163.37, 130.52, 130.49, 113.76, 55.64, 45.51, 41.41, 38.32, $38.25,36.93,35.57,30.04,28.75$.

LRMS (APCI) Calc. for $\left[\mathrm{C}_{16} \mathrm{H}_{20} \mathrm{O}_{2}\right]^{+}=244.15$, Found $=244.12$

HRMS (ESI) Calc. for $\left[\mathrm{C}_{16} \mathrm{H}_{20} \mathrm{O}_{2}+\mathrm{H}\right]^{+}=245.1536$, Found $=245.1539$

$\mathbf{R}_{\mathbf{f}}=0.6$ in $15 \%$ ethyl acetate/hexanes

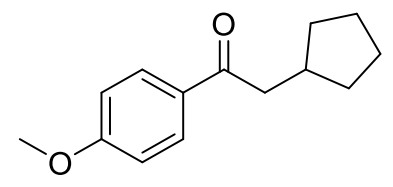

33 was synthesized via the general alkylation procedure above as a yellow oil (12 $\mathrm{mg}, 33 \%$ yield).

Analytical data for 33:

${ }^{1} \mathrm{H}-\mathrm{NMR}\left(300 \mathrm{MHz} ; \mathrm{CDCl}_{3}\right): \delta 7.91$ (d, J = $\left.9.02 \mathrm{~Hz}, 2 \mathrm{H}\right), 6.89(\mathrm{~d}, \mathrm{~J}=8.88,2 \mathrm{H}), 3.83(\mathrm{~s}, 3 \mathrm{H}), 2.90$ (d, J $=7.1 \mathrm{~Hz}, 2 \mathrm{H}), 2.45-2.25(\mathrm{~m}, 1 \mathrm{H}), 1.95-1.81(\mathrm{~m}, 2 \mathrm{H}), 1.68-1.41(\mathrm{~m}, 4 \mathrm{H}), 1.31-1.08(\mathrm{~m}, 2 \mathrm{H})$.

${ }^{13}$ C-NMR (126 MHz; $\left.\mathrm{CDCl}_{3}\right): \delta$ 199.27, 163.41, 130.55, 130.48, 113.75, 55.67, 44.64, 36.49, 32.90, 25.14 .

LRMS (APCI) Calc. for $\left[\mathrm{C}_{14} \mathrm{H}_{18} \mathrm{O}_{2}\right]^{+}=218.13$, Found $=218.14$

HRMS (ESI) Calc. for $\left[\mathrm{C}_{14} \mathrm{H}_{18} \mathrm{O}_{2}+\mathrm{H}\right]^{+}=219.1380$, Found $=219.1381$

$\mathbf{R}_{\mathbf{f}}=0.6$ in $15 \%$ ethyl acetate/hexanes 


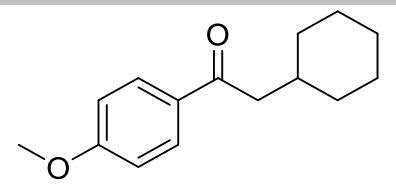

34 was synthesized via the general alkylation procedure above as a yellow oil (18 $\mathrm{mg}, 47 \%$ yield).

Analytical data for 34 :

${ }^{1} \mathrm{H}-N M R\left(300 \mathrm{MHz} ; \mathrm{CDCl}_{3}\right): \delta 7.94-7.85(\mathrm{~m}, 2 \mathrm{H}), 6.90(\mathrm{~d}, \mathrm{~J}=8.9 \mathrm{~Hz}, 2 \mathrm{H}), 3.84(\mathrm{~s}, 3 \mathrm{H}), 2.73(\mathrm{~d}, \mathrm{~J}=$ $6.8 \mathrm{~Hz}, 2 \mathrm{H}$ ), 1.92 (ddd, J = 11.1, 7.4, 3.6 Hz, 1H), 1.67 (dd, J = 28.3, $12.7 \mathrm{~Hz}, 4 \mathrm{H}), 1.37-0.88(\mathrm{~m}, 6 \mathrm{H})$.

${ }^{13} \mathrm{C}-\mathrm{NMR}\left(126 \mathrm{MHz} ; \mathrm{CDCl}_{3}\right): \delta$ 199.27, 163.41, 130.69, 130.57, 113.78, 55.62, 46.62, 36.42, 35.02, 28.48, 26.43.

LRMS (APCI) Calc. for $\left[\mathrm{C}_{15} \mathrm{H}_{20} \mathrm{O}_{2}\right]^{+}=232.15$, Found $=232.14$

HRMS (ESI) Calc. for $\left[\mathrm{C}_{15} \mathrm{H}_{20} \mathrm{O}_{2}+\mathrm{H}\right]^{+}=233.1536$, Found $=233.1536$

$\mathbf{R}_{\mathbf{f}}=0.5$ in $15 \%$ ethyl acetate/hexanes

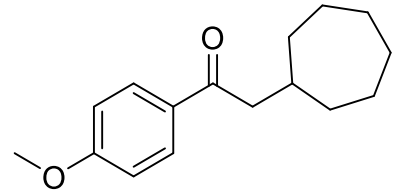

35 was synthesized via the general alkylation procedure as a yellow oil ( $24 \mathrm{mg}, 59 \%$ yield).

Analytical data for 35 :

${ }^{1} \mathrm{H}-\mathrm{NMR}\left(300 \mathrm{MHz} ; \mathrm{CDCl}_{3}\right): \delta 7.90$ (d, J = $\left.8.9 \mathrm{~Hz}, 2 \mathrm{H}\right), 6.89$ (d, J = 8.9 Hz, 2H), $3.83(\mathrm{~s}, 3 \mathrm{H}), 2.78$ (d, J $=6.9 \mathrm{~Hz}, 2 \mathrm{H}), 2.23-2.07(\mathrm{~m}, 1 \mathrm{H}), 1.80-1.37(\mathrm{~m}, 10 \mathrm{H}), 1.23(\mathrm{~m}, 9.7 \mathrm{~Hz}, 2 \mathrm{H})$.

${ }^{13}$ C-NMR (126 MHz; $\left.\mathrm{CDCl}_{3}\right): \delta$ 199.31, 163.39, 130.63, 130.58, 113.71, 55.69, 46.61, 36.40, 35.00, 28.47, 26.41.

LRMS (APCI) Calc. for $\left[\mathrm{C}_{16} \mathrm{H}_{22} \mathrm{O}_{2}\right]^{+}=246.16$, Found $=246.15$

HRMS (ESI) Calc. for $\left[\mathrm{C}_{9} \mathrm{H}_{10} \mathrm{O}_{2}+\mathrm{H}\right]^{+}=247.1693$, Found $=247.1692$

$\mathbf{R}_{\mathbf{f}}=0.5$ in $15 \%$ ethyl acetate/hexanes

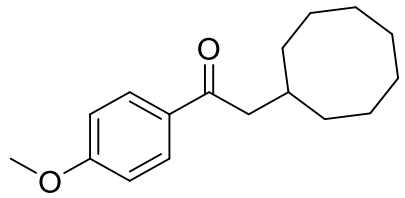

36 was synthesized via the general alkylation procedure above as a yellow oil (19 $\mathrm{mg}, 44 \%$ yield).

Analytical data for 36 :

${ }^{1} \mathrm{H}-\mathrm{NMR}\left(300 \mathrm{MHz} ; \mathrm{CDCl}_{3}\right): \delta 7.96(\mathrm{~d}, \mathrm{~J}=9.01 \mathrm{~Hz}, 2 \mathrm{H}), 6.95(\mathrm{~d}, \mathrm{~J}=8.80 \mathrm{~Hz}, 2 \mathrm{H}), 3.89(\mathrm{~s}, 3 \mathrm{H}), 2.83$ $(\mathrm{d}, \mathrm{J}=6.9 \mathrm{~Hz}, 2 \mathrm{H}), 2.33-2.16(\mathrm{~m}, 1 \mathrm{H}), 1.74-1.32(\mathrm{~m}, 14 \mathrm{H})$ 
${ }^{13} \mathrm{C}-\mathrm{NMR}\left(126 \mathrm{MHz} ; \mathrm{CDCl}_{3}\right): \delta$ 199.40, 163.37, 130.65, 130.55, 113.71, 55.55, 46.67, 34.47, 32.58, $27.27,26.26,25.42$

LRMS (APCI) Calc. for $\left[\mathrm{C}_{17} \mathrm{H}_{24} \mathrm{O}_{2}\right]^{+}=260.18$, Found $=261.22$

HRMS (ESI) Calc. for $\left[\mathrm{C}_{17} \mathrm{H}_{24} \mathrm{O}_{2}+\mathrm{H}\right]^{+}=261.1849$, Found $=261.1849$

$\mathbf{R}_{\mathbf{f}}=0.6$ in $15 \%$ ethyl acetate/hexanes

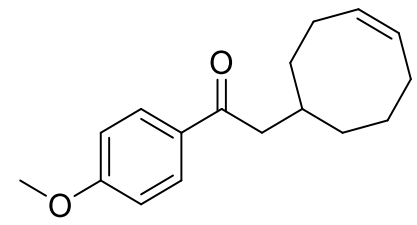

37 was synthesized via the general alkylation procedure above as a yellow oil ( $23 \mathrm{mg}, 54 \%$ yield).

Analytical data for $\mathbf{3 7}$ :

${ }^{1} \mathrm{H}-\mathrm{NMR}\left(300 \mathrm{MHz} ; \mathrm{CDCl}_{3}\right): \delta 7.96(\mathrm{~d}, \mathrm{~J}=7.5 \mathrm{~Hz}, 2 \mathrm{H}), 6.94(\mathrm{~d}, \mathrm{~J}=7.5 \mathrm{~Hz}, 2 \mathrm{H}), 5.76-5.51(\mathrm{~m}, 1 \mathrm{H})$, $3.89(\mathrm{~s}, 3 \mathrm{H}), 2.93-2.79(\mathrm{~m}, 2 \mathrm{H}), 2.44-2.01(\mathrm{~m}, 2 \mathrm{H}), 1.74-1.44(\mathrm{~m}, 5 \mathrm{H}), 1.45-1.66(\mathrm{~m}, 6 \mathrm{H})$

${ }^{13}$ C-NMR (126 MHz; $\left.\mathrm{CDCl}_{3}\right)$ : $\delta$ 199.01, 163.38, 130.67, 130.48, 130.43, 130.07, 113.80, 55.68, 47.42, $35.83,34.05,33.51,28.05,26.06,25.17$.

LRMS (APCI) Calc. for $\left[\mathrm{C}_{17} \mathrm{H}_{22} \mathrm{O}_{2}\right]^{+}=258.16$, Found $=258.16$

HRMS (ESI) Calc. for $\left[\mathrm{C}_{17} \mathrm{H}_{22} \mathrm{O}_{2}+\mathrm{H}\right]^{+}=259.1693$, Found $=259.1692$

$\mathbf{R}_{\mathbf{f}}=0.6$ in $15 \%$ ethyl acetate/hexanes
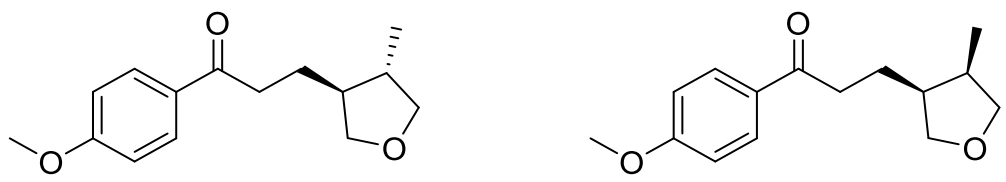

38 was synthesized via the general alkylation procedure ( $25 \mathrm{mg}, 61 \%$ yield) as a yellow oil which, by NMR, was determined to be a mixture of diastereomers with ratio of $57: 43$ favoring the trans product.

Analytical data for 38:

${ }^{1} \mathrm{H}-\mathrm{NMR}\left(300 \mathrm{MHz} ; \mathrm{CDCl}_{3}\right): \delta 7.90(\mathrm{~d}, \mathrm{~J}=8.9, \mathrm{~Hz}, 2 \mathrm{H}), 6.90(\mathrm{~d}, \mathrm{~J}=8.7 \mathrm{~Hz}, 2 \mathrm{H}), 4.06-3.78(\mathrm{~m}, 5 \mathrm{H})$, 3.46 (dd, J = 8.1, 2.4 Hz, 2H), 3.31 (dd, J = 18.2, $10.1 \mathrm{~Hz}, 2 \mathrm{H}$, cis-isomer), 2.88 (t, J = 7.6 Hz, 2H), $2.38-2.08(\mathrm{~m}, 1 \mathrm{H}), 2.05-1.55(\mathrm{~m}, 3 \mathrm{H}), 1.02(\mathrm{~d}, \mathrm{~J}=6.6 \mathrm{~Hz}, 3 \mathrm{H}$, cis-isomer), $0.94(\mathrm{~d}, \mathrm{~J}=6.9 \mathrm{~Hz}, 3 \mathrm{H})$

${ }^{13}$ C-NMR (126 MHz; $\left.\mathrm{CDCl}_{3}\right): \delta$ 198.58, 163.56, 130.43, 129.95, 129.92, 113.86, 75.39, 75.36, 73.82, $71.86,55.64,46.74,42.12,40.13,37.10,36.96,35.89,26.95,22.19,16.70,13.14$

LRMS (APCl) Calc. for $\left[\mathrm{C}_{15} \mathrm{H}_{20} \mathrm{O}_{3}+\mathrm{Na}\right]^{+}=271.13$, Found $=271.15$

HRMS (ESI) Calc. for $\left[\mathrm{C}_{15} \mathrm{H}_{20} \mathrm{O}_{3}+\mathrm{H}\right]^{+}=249.1485$, Found $=249.1483$

$\mathbf{R}_{\mathbf{f}}=0.2$ in $15 \%$ ethyl acetate/hexanes 

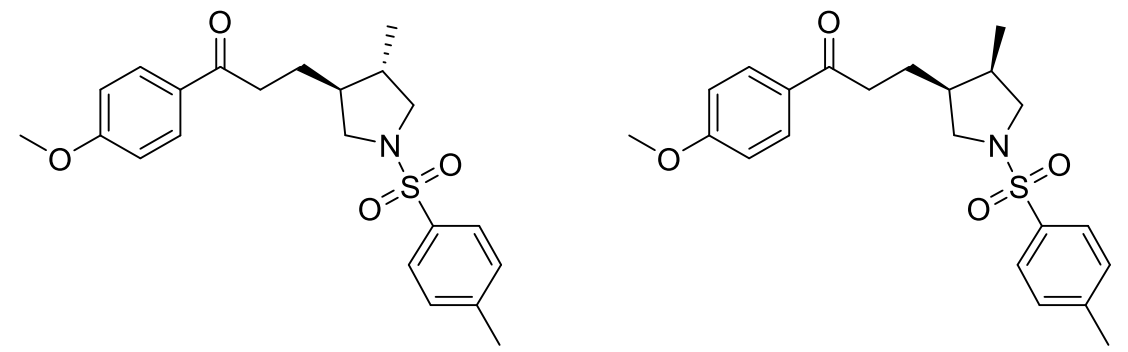

39 was synthesized via the general alkylation procedure (33 mg, $50 \%$ yield) as a yellow oil which, by NMR, was determined to be a near equal mixture of diastereomers with ratio of $51: 49$, slightly favoring the trans.

Analytical data for 39:

${ }^{1} \mathrm{H}-\mathrm{NMR}\left(300 \mathrm{MHz} ; \mathrm{CDCl}_{3}\right): \delta 7.96-7.87(\mathrm{~m}, 2 \mathrm{H}), 7.76-7.68(\mathrm{~m}, 2 \mathrm{H}), 7.37-7.25(\mathrm{~m}, 2 \mathrm{H}), 6.99-$ $6.91(\mathrm{~m}, 2 \mathrm{H}), 3.88(\mathrm{~s}, 3 \mathrm{H}), 3.60-3.31(\mathrm{~m}, 2 \mathrm{H}), 3.07-2.79(\mathrm{~m}, 2 \mathrm{H}), 2.43(\mathrm{~s}, 3 \mathrm{H}), 2.23$ (ddd, J = 9.9, 6.3, 3.1 Hz, 1H), $2.13-2.03(\mathrm{~m}, 1 \mathrm{H}$, cis-isomer), $1.70(\mathrm{~m}, 1 \mathrm{H}), 1.58-1.25(\mathrm{~m}, 2 \mathrm{H}), 1.03-0.94(\mathrm{~d}, \mathrm{~J}=$ $7.1 \mathrm{~Hz}, 3 \mathrm{H}$, cis-isoner), 0.77 (d, J = 7.0, 3H).

${ }^{13}$ C-NMR (126 MHz; $\left.\mathrm{CDCl}_{3}\right): \delta$ 197.99, 197.97, 163.64, 143.56, 143.52, 133.89, 55.67, 54.95, 53.41, 51.05, 45.38, 41.47, 39.18, 36.35, 36.34, 35.32, 26.25, 22.26, 21.72, 21.69, 16.36, 13.20.

HRMS (APCl) Calc. for $\left[\mathrm{C}_{22} \mathrm{H}_{27} \mathrm{NO}_{4} \mathrm{~S}+\mathrm{H}\right]^{+}=402.1739$, Found $=402.1732$

$\mathbf{R}_{\mathbf{f}}=0.1$ in $15 \%$ ethyl acetate/hexanes

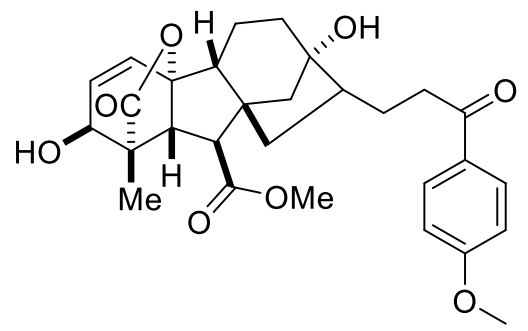

40 was synthesized via the general alkylation procedure as a colorless oil that, by NMR, was a single diastereomer (65 mg, 84\% yield).

Analytical data for $\mathbf{4 0}$ :

${ }^{1} \mathrm{H}-\mathrm{NMR}\left(300 \mathrm{MHz} ; \mathrm{CDCl}_{3}\right): \delta 7.95(\mathrm{~d}, \mathrm{~J}=9.02 \mathrm{~Hz}, 2 \mathrm{H}), 6.95(\mathrm{~d}, \mathrm{~J}=9.07 \mathrm{~Hz}, 2 \mathrm{H}), 6.33$ (d, J = $9.4 \mathrm{~Hz}$, $1 \mathrm{H}), 5.92(\mathrm{dd}, \mathrm{J}=9.3,3.7 \mathrm{~Hz}, 1 \mathrm{H}), 4.20-4.06(\mathrm{~m}, 1 \mathrm{H}), 3.89(\mathrm{~s}, 2 \mathrm{H}), 3.80-3.67(\mathrm{~m}, 2 \mathrm{H}), 3.19(\mathrm{t}, \mathrm{J}=$ $8.8 \mathrm{~Hz}, 1 \mathrm{H}), 3.08-2.89(\mathrm{~m}, 2 \mathrm{H}), 2.70(\mathrm{dd}, \mathrm{J}=28.8,10.5 \mathrm{~Hz}, 1 \mathrm{H}), 2.21-1.61(\mathrm{~m}, 12 \mathrm{H}), 1.36(\mathrm{ddd}, \mathrm{J}=$ 7.1, 6.2, $1.0 \mathrm{~Hz}, 2 \mathrm{H}), 1.24(\mathrm{~s}, 3 \mathrm{H})$. 
${ }^{13} \mathrm{C}-\mathrm{NMR}\left(126 \mathrm{MHz} ; \mathrm{CDCl}_{3}\right): \delta 199.21,178.81,173.10,163.66,132.88,132.70,130.55,129.90$, $113.88,90.74,78.74,69.89,60.62,55.66,53.65,53.54,52.74,52.38,52.09,51.87,46.64,46.36$, $42.42,36.97,30.40,26.15,21.27,16.75,14.57,14.35$

LRMS (APCl) Calc. for $\left[\mathrm{C}_{29} \mathrm{H}_{34} \mathrm{O}_{8}+\mathrm{Na}\right]^{+}=533.25$, Found $=533.22$

HRMS (ESI) Calc. for $\left[\mathrm{C}_{29} \mathrm{H}_{34} \mathrm{O}_{8}+\mathrm{H}\right]^{+}=511.2326$, Found $=511.2341$

$\mathbf{R}_{\mathbf{f}}=0.2$ in $15 \%$ ethyl acetate/hexanes

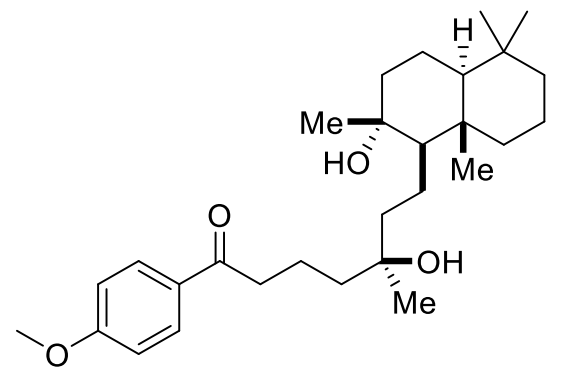

41 was synthesized via the general alkylation procedure as a colorless oil ( $36 \mathrm{mg}, 72 \%$ yield).

Analytical data for $\mathbf{4 1}$ :

${ }^{1} \mathrm{H}-\mathrm{NMR}\left(300 \mathrm{MHz} ; \mathrm{CDCl}_{3}\right): \delta 7.92(\mathrm{~d}, \mathrm{~J}=8.9 \mathrm{~Hz}, 1 \mathrm{H}), 6.90(\mathrm{~d}, \mathrm{~J}=8.9 \mathrm{~Hz}, 1 \mathrm{H}), 3.84(\mathrm{~s}, 2 \mathrm{H}), 3.77(\mathrm{~s}$, $1 \mathrm{H}), 2.91(\mathrm{t}, \mathrm{J}=7.2 \mathrm{~Hz}, 1 \mathrm{H}), 1.92-0.63(\mathrm{~m}, 31 \mathrm{H})$.

${ }^{13}$ C-NMR (126 MHz; $\left.\mathrm{CDCl}_{3}\right): \delta$ 199.25, 163.54, 125.85, 113.83, 113.59, 74.80, 73.43, 62.07, 56.17, $55.66,44.49$, 44.16, 42.14, 42.07, 39.85, 39.33, 38.41, 33.57, 33.40, 26.78, 24.53, 21.66, 20.66, 19.13, $18.93,18.58,15.61$.

HRMS (APCl) Calc. for $\left[\mathrm{C}_{29} \mathrm{H}_{46} \mathrm{O}_{4}+\mathrm{Na}\right]^{+}=481.3288$, Found $=481.3286$

$\mathbf{R}_{\mathbf{f}}=0.5$ in $15 \%$ ethyl acetate/hexanes

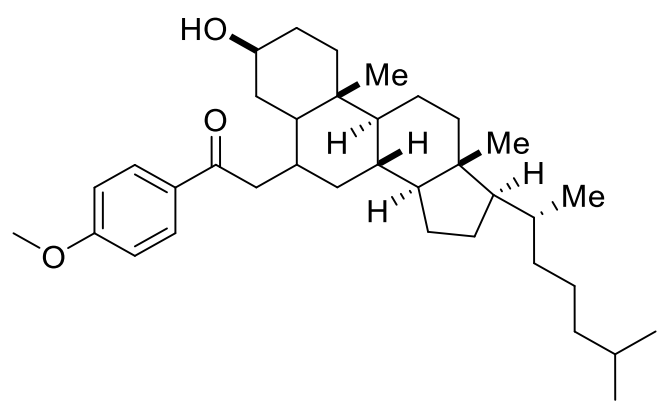

42 was synthesized via the general alkylation procedure as a single diastereomer as determined by ${ }^{1} \mathrm{H}$ and ${ }^{13} \mathrm{C}$ NMR, as a yellow oil $(25 \mathrm{mg}, 28 \%$ yield $)$

Analytical data for $\mathbf{4 2}$ : 
${ }^{1} \mathrm{H}-\mathrm{NMR}\left(300 \mathrm{MHz} ; \mathrm{CDCl}_{3}\right): \delta 7.89$ (d, J = 8.9 Hz, 2H), 6.90 (d, J = 8.9 Hz, 2H), 3.84 (s, 3H), 3.64 (td, $\mathrm{J}=10.7,5.6 \mathrm{~Hz}, 1 \mathrm{H}), 2.75-2.92(\mathrm{qd}, \mathrm{m}), 2.28(\mathrm{~d}, \mathrm{~J}=5.5 \mathrm{~Hz}, 1 \mathrm{H}), 2.07-1.88(\mathrm{~m}, 2 \mathrm{H}), 1.87-0.73(\mathrm{~m}$, $40 \mathrm{H}), 0.64(\mathrm{~s}, 3 \mathrm{H})$.

${ }^{13}$ C-NMR (126 MHz; $\left.\mathrm{CDCl}_{3}\right): \delta$ 199.37, 163.44, 130.55, 130.50, 113.82, 71.84, 56.38, 56.11, 55.65, $54.80,46.43$, 42.76, 40.06, 39.66, 39.49, 37.94, 36.82, 36.35, 36.28, 36.01, 35.95, 35.11, 31.69, 31.06, $28.33,28.18,24.41,23.96,23.01,22.74,21.29,18.82,16.67,12.35$.

HRMS (APCI) Calc. for $\left[\mathrm{C}_{36} \mathrm{H}_{56} \mathrm{O}_{3}+\mathrm{H}\right]^{+}=537.4302$, Found $=537.4297$

$\mathbf{R}_{\mathbf{f}}=0.4$ in $15 \%$ ethyl acetate/hexanes<smiles>COc1ccc(C(=O)C[C@H](CCC(C)CCO)C(C)C)cc1</smiles>

43 was synthesized via the general alkylation procedure (22 mg, 44\% yield) as clear oil that, by NMR, was found to be a mixture of diastereomers in ratio of 66:33.

Analytical data for 43:

${ }^{1} \mathrm{H}-\mathrm{NMR}\left(300 \mathrm{MHz} ; \mathrm{CDCl}_{3}\right): \delta 7.91(\mathrm{~d}, \mathrm{~J}=8.9,2 \mathrm{H}), 6.90(\mathrm{~d}, \mathrm{~J}=8.9 \mathrm{~Hz}, 2 \mathrm{H}), 3.84(\mathrm{~s}, 3 \mathrm{H}), 3.61(\mathrm{dt}, \mathrm{J}=$ 6.9, $4.3 \mathrm{~Hz}, 2 \mathrm{H}), 2.85$ (ddd, J = 16.0, 5.4, $3.5 \mathrm{~Hz}, 1 \mathrm{H}), 2.75-2.60(\mathrm{~m}, 1 \mathrm{H}), 2.03-1.93(\mathrm{~m}, 1 \mathrm{H}), 1.81-$ $1.01(\mathrm{~m}, 8 \mathrm{H}), 0.92-0.79(\mathrm{~m}, 9 \mathrm{H})$.

${ }^{13}$ C-NMR (126 MHz; $\left.\mathrm{CDCl}_{3}\right): \delta$ 199.92, 199.81, 163.38, 130.64, 130.51, 113.80, 61.29, 61.14, 55.63, 40.37, 40.03, 39.95, 39.89, 39.74, 34.87, 34.55, 30.10, 29.94, 29.71, 29.34, 28.78, 28.60, 19.92, 19.75, $18.86,18.52$.

LRMS $(\mathrm{APCl})$ Calc. for $\left[\mathrm{C}_{19} \mathrm{H}_{30} \mathrm{O}_{3}+\mathrm{H}\right]^{+}=307.22$, Found $=307.21$

HRMS (ESI) Calc. for $\left[\mathrm{C}_{19} \mathrm{H}_{30} \mathrm{O}_{3}+\mathrm{H}\right]^{+}=307.2268$, Found $=307.2266$

$\mathbf{R}_{\mathbf{f}}=0.1$ in $15 \%$ ethyl acetate/hexanes<smiles>COc1ccc(C(=O)CC[C@H]2O[C@H](OC)[C@@H](OC(C)=O)[C@H](OC(C)=O)[C@H]2OC(C)=O)cc1</smiles>

44 was synthesized via the general alkylation procedure above as a single diastereomer (>95:5) as determined by NMR analysis as a white solid ( $51 \mathrm{mg}, 68 \%$ yield).

Analytical data for 44: 
${ }^{1} \mathrm{H}-N M R\left(300 \mathrm{MHz} ; \mathrm{C}_{6} \mathrm{D}_{6}\right): \delta 7.92(\mathrm{~d}, \mathrm{~J}=8.9 \mathrm{~Hz}, 2 \mathrm{H}), 6.66(\mathrm{~d}, \mathrm{~J}=8.9 \mathrm{~Hz}, 2 \mathrm{H}), 5.89$ (dd, J = 10.1, 9.5 $\mathrm{Hz}, 1 \mathrm{H}), 5.89(\mathrm{dd}, \mathrm{J}=10.1,9.5 \mathrm{~Hz}, 1 \mathrm{H}), 5.17(\mathrm{t}, \mathrm{J}=9.83 \mathrm{~Hz}, 1 \mathrm{H}), 5.09(\mathrm{dd}, \mathrm{J}=10.3,3.7 \mathrm{~Hz}, 1 \mathrm{H}), 4.89$ (d, J = 3.6 Hz, 1H), 3.92 (ddd, J = 10.0, 8.9, 3.0 Hz, 1H), $3.20(\mathrm{~s}, 2 \mathrm{H}), 2.98-2.85(\mathrm{~m}, 5 \mathrm{H}), 2.27$ (dtd, J $=10.9,7.6,3.0 \mathrm{~Hz}, 1 \mathrm{H}), 1.97-1.79(\mathrm{~m}, 1 \mathrm{H}), 1.74(\mathrm{~s}, 3 \mathrm{H}), 1.69(\mathrm{~s}, 3 \mathrm{H}), 1.63(\mathrm{~s}, 3 \mathrm{H})$.

${ }^{13}$ C-NMR (126 MHz; $\left.\mathrm{CDCl}_{3}\right): \delta$ 197.98, 170.46, 170.26, 170.15, 163.59, 130.42, 129.90, 113.86, 96.59, $72.15,71.28,70.24,67.60,55.65,55.37,33.18,25.54,20.99,20.95,20.91$

HRMS (APCI) Calc. for $\left[\mathrm{C}_{22} \mathrm{H}_{28} \mathrm{O}_{10}+\mathrm{H}\right]^{+}=453.1755$, Found $=453.1751$

$\mathbf{R}_{\mathbf{f}}=0.6$ in $15 \%$ ethyl acetate/hexanes

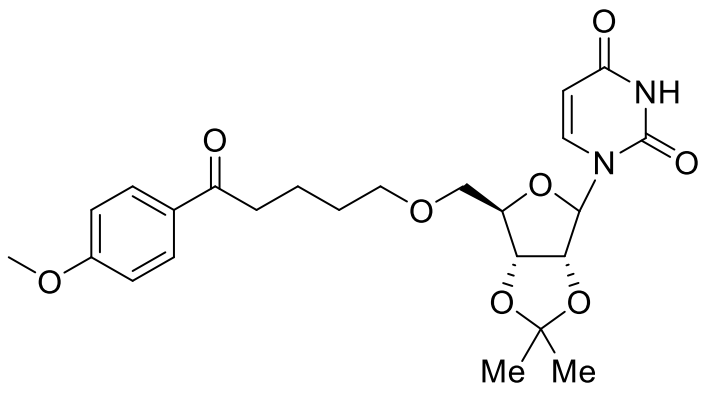

45 was synthesized via the general alkylation procedure as a colorless oil ( $35 \mathrm{mg}, 67 \%$ yield).

Analytical data for $\mathbf{4 5}$ :

${ }^{1} \mathrm{H}-\mathrm{NMR}\left(300 \mathrm{MHz} ; \mathrm{CDCl}_{3}\right): \delta 8.00-7.92(\mathrm{~m}, 2 \mathrm{H}), 7.31(\mathrm{~d}, \mathrm{~J}=8.1 \mathrm{~Hz}, 2 \mathrm{H}), 6.99-6.87(\mathrm{~m}, 2 \mathrm{H}), 5.78$ $(\mathrm{d}, \mathrm{J}=8.0 \mathrm{~Hz}, 1 \mathrm{H}), 5.53(\mathrm{~d}, \mathrm{~J}=2.8 \mathrm{~Hz}, 1 \mathrm{H}), 5.05$ (ddd, J = 33.5, 6.5, 3.1 Hz, 2H), 4.33 (dd, J = 6.0, 3.5 $\mathrm{Hz}, 1 \mathrm{H}), 4.03-3.81(\mathrm{~m}, 7 \mathrm{H}), 2.99(\mathrm{t}, \mathrm{J}=6.4 \mathrm{~Hz}, 2 \mathrm{H}), 1.79-1.73(\mathrm{~m}, 4 \mathrm{H}), 1.60(\mathrm{~s}, 3 \mathrm{H}), 1.40-1.31(\mathrm{~m}$, $3 \mathrm{H})$.

${ }^{13} \mathrm{C}-\mathrm{NMR}\left(126 \mathrm{MHz} ; \mathrm{CDCl}_{3}\right): \delta 198.82,163.51,162.65,151.10,141.02,130.51,130.09,114.42$, $113.83,102.30,97.65,87.35,83.91,80.56,63.00,55.64,40.89,37.83,27.42,27.23,25.40,21.67$, 14.38.

LRMS (APCI) Calc. for $\left[\mathrm{C}_{24} \mathrm{H}_{30} \mathrm{~N}_{2} \mathrm{O}_{8}+\mathrm{Na}\right]^{+}=474.25$, Found $=497.29$

HRMS (ESI) Calc. for $\left[\mathrm{C}_{24} \mathrm{H}_{30} \mathrm{~N}_{2} \mathrm{O}_{8}+\mathrm{Na}\right]^{+}=497.1894$, Found $=497.1887$

$\mathbf{R}_{\mathbf{f}}=0.3$ in ethyl acetate 


\section{G. Characterization of Alkylation Products in Thiol Scope}

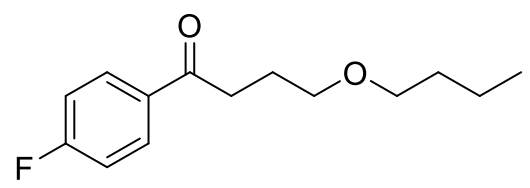

46a was synthesized via the general alkylation procedure (37 mg, 94\% yield) as a colorless oil.

Analytical data for 46a:

${ }^{1} \mathrm{H}-\mathrm{NMR}\left(300 \mathrm{MHz} ; \mathrm{CDCl}_{3}\right): \delta 8.02-7.90(\mathrm{~m}, 2 \mathrm{H}), 7.15-7.02(\mathrm{t}, \mathrm{J}=8.62 \mathrm{~Hz}, 2 \mathrm{H}), 3.45(\mathrm{t}, \mathrm{J}=6.1 \mathrm{~Hz}$, $2 \mathrm{H}), 3.37(\mathrm{t}, \mathrm{J}=6.6 \mathrm{~Hz}, 2 \mathrm{H}), 3.01(\mathrm{t}, \mathrm{J}=7.2 \mathrm{~Hz}, 2 \mathrm{H}), 2.06-1.91(\mathrm{~m}, 2 \mathrm{H}), 1.49$ (quint, $\mathrm{J}=7.2 \mathrm{~Hz}, 2 \mathrm{H}$ ), 1.32 (quint, , $2 \mathrm{H}), 0.87(\mathrm{t}, \mathrm{J}=7.3 \mathrm{~Hz}, 3 \mathrm{H}$ ).

${ }^{13}$ C-NMR (126 MHz; $\mathrm{CDCl}_{3}$ ): $\delta$ 198.68, 165.75 (d), 133.61 (d), 130.83 (d), 115.75 (d), 70.84, 69.82, $35.28,31.97,24.50,19.53,14.11$.

${ }^{19}$ F-NMR $\left(282 \mathrm{MHz} ; \mathrm{CDCl}_{3}\right): \delta 105.68(\mathrm{~m})$

LRMS (APCI) Calc. for $\left[\mathrm{C}_{14} \mathrm{H}_{19} \mathrm{FO}_{2}\right]^{+}=238.14$, Found $=238.14$

HRMS (ESI) Calc. for $\left[\mathrm{C}_{14} \mathrm{H}_{19} \mathrm{FO}_{2}+\mathrm{H}\right]^{+}=239.1442$, Found $=239.1444$

$\mathbf{R}_{\mathbf{f}}=0.5$ in $15 \%$ ethyl acetate/hexanes

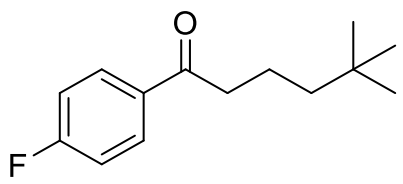

46b was synthesized via the general alkylation procedure (17 mg, $45 \%$ yield) as a colorless oil.

Analytical data for $\mathbf{4 6 b}$ :

${ }^{1} \mathrm{H}-\mathrm{NMR}\left(300 \mathrm{MHz} ; \mathrm{CDCl}_{3}\right): \delta 7.95$ (dd, J = 8.8, $\left.5.4 \mathrm{~Hz}, 2 \mathrm{H}\right), 7.10(\mathrm{t}, \mathrm{J}=8.6 \mathrm{~Hz}, 2 \mathrm{H}), 2.88$ (t, J = 7.4 $\mathrm{Hz}, 2 \mathrm{H}), 1.76-1.60(\mathrm{~m}, 2 \mathrm{H}), 1.25-1.18(\mathrm{~m}, 2 \mathrm{H}), 0.87(\mathrm{~s}, 9 \mathrm{H})$.

${ }^{13}$ C-NMR (126 MHz; $\mathrm{CDCl}_{3}$ ): $\delta$ 199.12, 165.80 (d), 133.72 (d), 130.81 (d), 115.80 (d), 44.02, 39.51, $29.50,19.76,16.11$

${ }^{19}$ F-NMR (282 MHz; $\left.\mathrm{CDCl}_{3}\right): \delta 105.74(\mathrm{~m})$

LRMS $(\mathrm{APCl})$ Calc. for $\left[\mathrm{C}_{14} \mathrm{H}_{19} \mathrm{FO}\right]^{+}=222.14$, Found $=222.10$

HRMS (ESI) Calc. for $\left[\mathrm{C}_{14} \mathrm{H}_{19} \mathrm{FO}+\mathrm{H}\right]^{+}=223.1493$, Found $=223.1489$

$\mathbf{R}_{\mathbf{f}}=0.6$ in $15 \%$ ethyl acetate/hexanes 


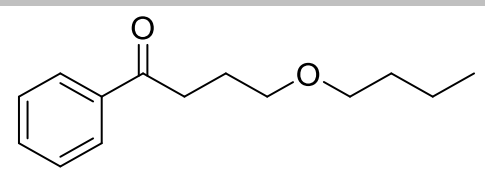

47a was synthesized via the general alkylation procedure (36 mg, 96\% yield) as a yellow oil.

Analytical data for $47 a$ :

${ }^{1} \mathrm{H}-N M R\left(300 \mathrm{MHz} ; \mathrm{CDCl}_{3}\right): \delta 7.99-7.89(\mathrm{~m}, 2 \mathrm{H}), 7.59-7.47(\mathrm{~m}, 1 \mathrm{H}), 7.47-7.37(\mathrm{~m}, 2 \mathrm{H}), 3.47(\mathrm{t}, \mathrm{J}$ $=6.1 \mathrm{~Hz}, 2 \mathrm{H}), 3.38(\mathrm{t}, \mathrm{J}=6.6 \mathrm{~Hz}, 2 \mathrm{H}), 3.05(\mathrm{t}, \mathrm{J}=7.2 \mathrm{~Hz}, 2 \mathrm{H}), 2.05-1.90(\mathrm{~m}, 2 \mathrm{H}), 1.50$ (quint, $\mathrm{J}=7.2$ $\mathrm{Hz}, 2 \mathrm{H}$ ), 1.33 (quint, $\mathrm{J}=7.3 \mathrm{~Hz}, 2 \mathrm{H}$ ), 0.87 (t, J = 7.3 Hz, 3H).

${ }^{13}$ C-NMR (126 MHz; $\left.\mathrm{CDCl}_{3}\right): \delta$ 200.32, 137.14, 133.12, 128.70, 128.21, 70.84, 69.91, 35.35, 31.97, $24.46,19.54,14.14$

LRMS (APCI) Calc. for $\left[\mathrm{C}_{14} \mathrm{H}_{20} \mathrm{O}_{2}\right]^{+}=220.15$, Found $=220.19$

HRMS (ESI) Calc. for $\left[\mathrm{C}_{14} \mathrm{H}_{20} \mathrm{O}_{2}+\mathrm{Na}\right]^{+}=243.1356$, Found $=243.1355$

$\mathbf{R}_{\mathbf{f}}=0.7$ in $15 \%$ ethyl acetate/hexanes

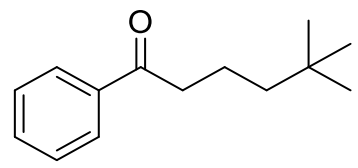

47b was synthesized via the general alkylation procedure ( $15 \mathrm{mg}, 43 \%$ yield) as a yellow oil.

Analytical data for $\mathbf{4 7 b}$ :

${ }^{1} \mathrm{H}-N M R\left(300 \mathrm{MHz} ; \mathrm{CDCl}_{3}\right): \delta 7.99-7.89(\mathrm{~m}, 2 \mathrm{H}), 7.59-7.47(\mathrm{~m}, 1 \mathrm{H}), 7.47-7.37(\mathrm{~m}, 2 \mathrm{H}), 3.47(\mathrm{t}, \mathrm{J}$ $=6.1 \mathrm{~Hz}, 2 \mathrm{H}$ ), $3.38(\mathrm{t}, \mathrm{J}=6.6 \mathrm{~Hz}, 2 \mathrm{H}), 3.05(\mathrm{t}, \mathrm{J}=7.2 \mathrm{~Hz}, 2 \mathrm{H}), 2.05-1.90(\mathrm{~m}, 2 \mathrm{H}), 1.50$ (quint, J = 7.2 $\mathrm{Hz}, 2 \mathrm{H}$ ), 1.33 (quint, $\mathrm{J}=7.3 \mathrm{~Hz}, 2 \mathrm{H}$ ), $0.87(\mathrm{t}, \mathrm{J}=7.3 \mathrm{~Hz}, 3 \mathrm{H}$ ).

${ }^{13}$ C-NMR (126 MHz; $\left.\mathrm{CDCl}_{3}\right): \delta$ 200.32, 137.14, 133.12, 128.70, 128.21, 70.84, 69.91, 35.35, 31.97, $24.46,19.54,14.14$

LRMS (APCl) Calc. for $\left[\mathrm{C}_{14} \mathrm{H}_{20} \mathrm{O}+\mathrm{H}\right]^{+}=205.16$, Found $=205.14$

HRMS (ESI) Calc. for $\left[\mathrm{C}_{14} \mathrm{H}_{20} \mathrm{O}+\mathrm{H}\right]^{+}=205.1587$, Found $=205.1582$

$\mathbf{R}_{\mathbf{f}}=0.7$ in $15 \%$ ethyl acetate/hexanes 


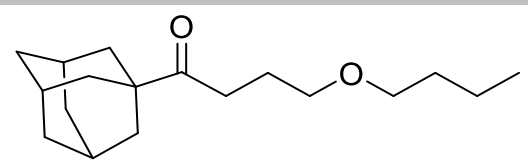

48a was synthesized via the general alkylation procedure (44 mg, 98\% yield) as a white solid.

Analytical data for $\mathbf{4 8 a}$ :

${ }^{1} \mathrm{H}-\mathrm{NMR}\left(300 \mathrm{MHz} ; \mathrm{CDCl}_{3}\right): \delta 3.33(\mathrm{t}, \mathrm{J}=6.4 \mathrm{~Hz}, 4 \mathrm{H}), 2.49(\mathrm{t}, \mathrm{J}=7.1 \mathrm{~Hz}, 2 \mathrm{H}), 1.99(\mathrm{~s}, 3 \mathrm{H}), 1.80-$ $1.57(\mathrm{~m}, 12 \mathrm{H}), 1.49(\mathrm{~m}, 2 \mathrm{H}), 1.31(\mathrm{~m}, 2 \mathrm{H}), 0.87(\mathrm{t}, \mathrm{J}=7.3 \mathrm{~Hz}, 4 \mathrm{H})$.

${ }^{13}$ C-NMR (126 MHz; $\left.\mathrm{CDCl}_{3}\right): \delta$ 215.66, 70.72, 69.98, 46.43, 38.34, 36.73, 32.69, 31.95, 28.10, 23.87, $19.52,14.10$

LRMS $(\mathrm{APCl})$ Calc. for $\left[\mathrm{C}_{18} \mathrm{H}_{30} \mathrm{O}_{2}+\mathrm{H}\right]^{+}=279.23$, Found $=279.20$

HRMS (ESI) Calc. for $\left[\mathrm{C}_{18} \mathrm{H}_{30} \mathrm{OS}+\mathrm{H}\right]^{+}=279.2319$, Found $=279.2314$

$\mathbf{R}_{\mathbf{f}}=0.6$ in $15 \%$ ethyl acetate/hexanes

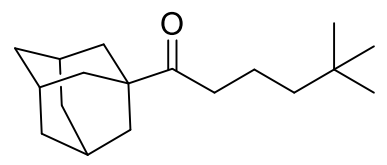

48b was synthesized via the general alkylation ( $34 \mathrm{mg}, 75 \%$ yield) as a white solid.

Analytical data for $\mathbf{4 8 b}$ :

${ }^{1} \mathrm{H}-\mathrm{NMR}\left(300 \mathrm{MHz} ; \mathrm{CDCl}_{3}\right): \delta 2.37(\mathrm{t}, \mathrm{J}=7.3 \mathrm{~Hz}, 2 \mathrm{H}), 2.00(\mathrm{~s}, 3 \mathrm{H}), 1.81-1.59(\mathrm{~m}, 12 \mathrm{H}), 1.45$ (ddd, J $=12.4,7.2,4.3 \mathrm{~Hz}, 2 \mathrm{H}), 1.12-1.03(\mathrm{~m}, 2 \mathrm{H}), 0.84(\mathrm{~s}, 9 \mathrm{H})$.

${ }^{13}$ C-NMR (126 MHz; $\left.\mathrm{CDCl}_{3}\right): \delta$ 216.20, 46.41, 44.01, 38.39, 36.93, 36.78, 30.57, 29.51, 28.14, 19.01

LRMS $(\mathrm{APCl})$ Calc. for $\left[\mathrm{C}_{18} \mathrm{H}_{30} \mathrm{O}+\mathrm{H}\right]^{+}=263.24$, Found $=263.26$

HRMS (APCl) Calc. for $\left[\mathrm{C}_{18} \mathrm{H}_{30} \mathrm{O}+\mathrm{H}\right]^{+}=263.2371$, Found $=263.2369$

$\mathbf{R}_{\mathbf{f}}=0.7$ in $15 \%$ ethyl acetate/hexanes

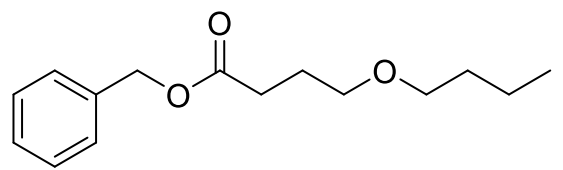

49a was synthesized via the general alkylation procedure ( $28 \mathrm{mg}, 68 \%$ yield) as a colorless oil. Analytical data for 49a: 
${ }^{1} \mathrm{H}-\mathrm{NMR}\left(300 \mathrm{MHz} ; \mathrm{CDCl}_{3}\right): \delta 7.32(\mathrm{~s}, 5 \mathrm{H}), 5.09(\mathrm{~s}, 2 \mathrm{H}), 3.40(\mathrm{t}, \mathrm{J}=6.7 \mathrm{~Hz}, 2 \mathrm{H}), 3.35$ (t, J = $6.7 \mathrm{~Hz}$, $2 \mathrm{H}$ ), $2.43(\mathrm{t}, \mathrm{J}=7.4 \mathrm{~Hz}, 2 \mathrm{H}$ ), 1.89 (quint, $\mathrm{J}=6.8 \mathrm{~Hz}, 2 \mathrm{H}$ ), 1.49 (quint, $\mathrm{J}=6.74 \mathrm{~Hz}, 2 \mathrm{H}$ ), 1.32 (quint, $\mathrm{J}=$ $8.04 \mathrm{~Hz}, 2 \mathrm{H}), 0.87(\mathrm{t}, \mathrm{J}=7.3 \mathrm{~Hz}, 2 \mathrm{H})$.

${ }^{13}$ C-NMR (126 MHz; $\left.\mathrm{CDCl}_{3}\right): \delta$ 73.55, 136.20, 128.69, 128.33, 77.45, 77.20, 76.95, 70.85, 69.69, $66.31,31.94,31.26,25.25,19.50,14.11$.

LRMS (APCI) Calc. for $\left[\mathrm{C}_{15} \mathrm{H}_{22} \mathrm{O}_{3}\right]^{+}=250.15$, Found $=250.11$

HRMS (ESI) Calc. for $\left[\mathrm{C}_{15} \mathrm{H}_{22} \mathrm{O}_{3}+\mathrm{Na}\right]^{+}=273.1461$, Found $=273.1457$

$\mathbf{R}_{\mathbf{f}}=0.3$ in $15 \%$ ethyl acetate/hexanes

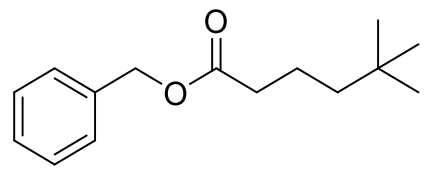

49b was synthesized via the general alkylation procedure (20 mg, $52 \%$ yield) as a colorless oil.

Analytical data for $49 b$ :

${ }^{1} \mathrm{H}-\mathrm{NMR}\left(300 \mathrm{MHz} ; \mathrm{CDCl}_{3}\right): \delta 7.36-7.26(\mathrm{~m}, 5 \mathrm{H}), 5.09(\mathrm{~s}, 2 \mathrm{H}), 2.30(\mathrm{t}, \mathrm{J}=7.5 \mathrm{~Hz}, 2 \mathrm{H}), 1.68-1.51$ $(\mathrm{m}, 2 \mathrm{H}), 1.19-1.08(\mathrm{~m}, 2 \mathrm{H}), 0.84(\mathrm{~s}, 9 \mathrm{H})$.

${ }^{13} \mathrm{C}-\mathrm{NMR}\left(126 \mathrm{MHz} ; \mathrm{CDCl}_{3}\right): \delta$ 173.84, 136.29, 128.70, 128.39, 128.34, 66.24, 43.71, 35.24, 29.44, $20.44,16.12,16.06$.

LRMS (APCI) Calc. for $\left[\mathrm{C}_{15} \mathrm{H}_{22} \mathrm{O}_{2}\right]^{+}=234.16$, Found $=234.15$

HRMS (ESI) Calc. for $\left[\mathrm{C}_{15} \mathrm{H}_{22} \mathrm{O}_{2}+\mathrm{Na}\right]^{+}=273.1461$, Found $=273.1457$

$\mathbf{R}_{\mathbf{f}}=0.5$ in $15 \%$ ethyl acetate/hexanes

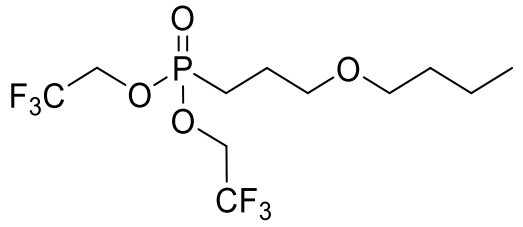

50a was synthesized via the general alkylation procedure (34 $\mathrm{mg}, 60 \%$ yield) as a yellow oil.

Analytical data for $\mathbf{5 0 a}$

${ }^{1} \mathrm{H}-\mathrm{NMR}\left(300 \mathrm{MHz} ; \mathrm{CDCl}_{3}\right): \delta 4.42-4.24(\mathrm{~m}, 1 \mathrm{H}), 3.47-3.28(\mathrm{~m}, 1 \mathrm{H}), 2.13-1.75(\mathrm{~m}, 1 \mathrm{H}), 1.50$ (quin, $\mathrm{J}=7.40 \mathrm{~Hz}, 2 \mathrm{H}$ ), 1.32 (quin, $\mathrm{J}=8.0 \mathrm{~Hz}, 2 \mathrm{H}$ ), $0.88(\mathrm{t}, \mathrm{J}=7.3 \mathrm{~Hz}, 1 \mathrm{H}$ ).

${ }^{13} \mathrm{C}-N M R\left(126 \mathrm{MHz} ; \mathrm{CDCl}_{3}\right.$ ): $\delta 122.87$ (qd), 70.91, 69.71 (d), 62.05 (qd), 31.91, 23.26, 22.57, 22.53, $22.12,19.49,14.04$

${ }^{31}$ P-NMR (121 MHz; $\left.\mathrm{CDCl}_{3}\right): \delta 36.12(\mathrm{~m})$ 
${ }^{19}$ F-NMR (282 MHz; $\left.\mathrm{CDCl}_{3}\right): \delta 75.36(\mathrm{t})$

Elemental Analysis for $\mathrm{C}_{11} \mathrm{H}_{19} \mathrm{~F}_{6} \mathrm{O}_{4} \mathrm{P}$ : Calc. C, 36.68; $\mathrm{H}, 5.32 ; \mathrm{N}, 0.0$. Found: $\mathrm{C}, 37.23 ; \mathrm{H}, 5.15 ; \mathrm{N}$, $<0.10$.

$\mathbf{R}_{\mathbf{f}}=0.6$ in $15 \%$ ethyl acetate/hexanes

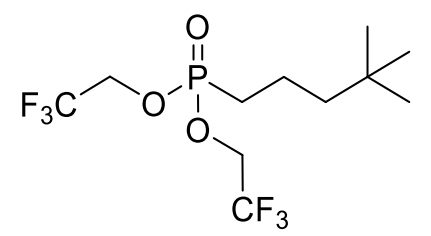

50b was synthesized via the general alkylation procedure ( $30 \mathrm{mg}, 55 \%$ yield) as a yellow oil.

Analytical data for $\mathbf{5 0 b}$ :

${ }^{1} \mathrm{H}-\mathrm{NMR}\left(300 \mathrm{MHz} ; \mathrm{CDCl}_{3}\right): \delta 4.45-4.27(\mathrm{~m}, 4 \mathrm{H}), 1.93-1.72(\mathrm{~m}, 2 \mathrm{H}), 1.69-1.46(\mathrm{~m}, 2 \mathrm{H}), 1.33-$ $1.18(\mathrm{~m}, 2 \mathrm{H}), 0.85(\mathrm{~s}, 9 \mathrm{H})$.

${ }^{13}$ C-NMR (126 MHz; $\mathrm{CDCl}_{3}$ ): $\delta 122.39$ (dq), 62.00 (qd), 45.11 (d), 29.33 (s), 27.12 (s), 26.01 (s), 17.43 (d)

${ }^{31}$ P-NMR (121 MHz; $\left.\mathrm{CDCl}_{3}\right): \delta$ 35.98(m)

${ }^{19} \mathrm{~F}-\mathrm{NMR}\left(282 \mathrm{MHz} ; \mathrm{CDCl}_{3}\right): \delta 75.32(\mathrm{t})$

Elemental Analysis for $\mathrm{C}_{11} \mathrm{H}_{19} \mathrm{~F}_{6} \mathrm{O}_{3} \mathrm{P}$ : Calc. $\mathrm{C}, 38.38 ; \mathrm{H}, 5.56 ; \mathrm{N}, 0.0$. Found: $\mathrm{C}, 40.89 ; \mathrm{H}, 5.79 ; \mathrm{N}$, 0.68 .

$\mathbf{R}_{\mathbf{f}}=0.3$ in $25 \%$ diethyl ether/hexanes

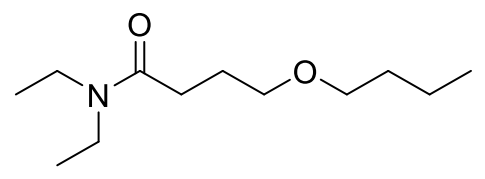

51a was synthesized via the general alkylation procedure (18 $\mathrm{mg}, 47 \%$ yield) as a yellow oil.

Analytical data for 51a:

${ }^{1} \mathrm{H}-\mathrm{NMR}\left(300 \mathrm{MHz} ; \mathrm{CDCl}_{3}\right): \delta 3.46-3.18(\mathrm{~m}, 8 \mathrm{H}), 2.36(\mathrm{t}, \mathrm{J}=7.3 \mathrm{~Hz}, 2 \mathrm{H}), 1.96-1.80(\mathrm{~m}, 2 \mathrm{H}), 1.50$ $(\mathrm{d}, \mathrm{J}=7.9 \mathrm{~Hz}, 2 \mathrm{H}), 1.33(\mathrm{~d}, \mathrm{~J}=7.6 \mathrm{~Hz}, 2 \mathrm{H}), 1.13(\mathrm{t}, \mathrm{J}=7.1 \mathrm{~Hz}, 3 \mathrm{H}), 1.07(\mathrm{t}, \mathrm{J}=7.1 \mathrm{~Hz}, 3 \mathrm{H}), 0.88(\mathrm{t}, \mathrm{J}$ $=7.3 \mathrm{~Hz}, 6 \mathrm{H})$.

${ }^{13} \mathrm{C}-\mathrm{NMR}\left(126 \mathrm{MHz} ; \mathrm{CDCl}_{3}\right): \delta 172.03,70.73,70.07,42.07,40.26,32.03,29.68,25.69,19.56,14.50$, 14.12, 13.32 .

LRMS (APCI) Calc. for $\left[\mathrm{C}_{12} \mathrm{H}_{15} \mathrm{NO}_{2}\right]^{+}=215.19$, Found $=215.17$ 
HRMS (ESI) Calc. for $\left[\mathrm{C}_{12} \mathrm{H}_{15} \mathrm{NO}_{2}+\mathrm{H}\right]^{+}=216.1958$, Found $=216.1962$

$\mathbf{R}_{\mathbf{f}}=0.2$ in $15 \%$ ethyl acetate/hexanes

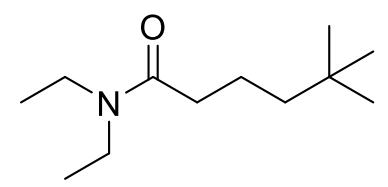

51b was synthesized via the general alkylation procedure (14 $\mathrm{mg}, 40 \%$ yield) as a yellow oil.

Analytical data for $\mathbf{5 1 b}$

${ }^{1} \mathrm{H}-\mathrm{NMR}\left(300 \mathrm{MHz} ; \mathrm{CDCl}_{3}\right): \delta \delta 3.33(\mathrm{dd}, \mathrm{J}=14.5,7.3 \mathrm{~Hz}, 1 \mathrm{H}), 3.26(\mathrm{dd}, \mathrm{J}=14.6,7.4 \mathrm{~Hz}, 1 \mathrm{H}), 2.25-$ $2.18(\mathrm{~m}, 1 \mathrm{H}), 1.64-1.50(\mathrm{~m}, 1 \mathrm{H}), 1.12(\mathrm{ddd}, \mathrm{J}=17.3,15.7,9.7 \mathrm{~Hz}, 4 \mathrm{H}), 0.85(\mathrm{~s}, 5 \mathrm{H})$.

${ }^{13} \mathrm{C}-\mathrm{NMR}\left(126 \mathrm{MHz} ; \mathrm{CDCl}_{3}\right): \delta 172.46,44.19,42.11,40.20,34.12,30.57,29.54,20.89,14.56,13.31$

LRMS (APCI) Calc. for $\left[\mathrm{C}_{12} \mathrm{H}_{25} \mathrm{NO}\right]^{+}=199.19$, Found $=199.21$

HRMS (ESI) Calc. for $\left[\mathrm{C}_{12} \mathrm{H}_{25} \mathrm{NO}+\mathrm{H}\right]^{+}=200.2009$, Found $=200.2013$

$\mathbf{R}_{\mathbf{f}}=0.1$ in $15 \%$ ethyl acetate/hexanes

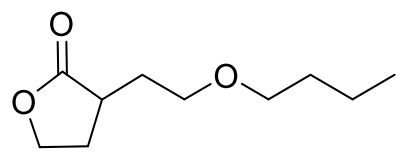

52a was synthesized via the general alkylation procedure (18 $\mathrm{mg}, 32 \%$ yield) as a yellow oil.

Analytical data for 52a:

${ }^{1} \mathrm{H}-\mathrm{NMR}\left(300 \mathrm{MHz} ; \mathrm{CDCl}_{3}\right): \delta 4.30$ (dd, J = 8.7, $\left.2.6 \mathrm{~Hz}, 1 \mathrm{H}\right), 4.15$ (ddd, J = 9.9, 9.0, $\left.6.6 \mathrm{~Hz}, 1 \mathrm{H}\right), 3.50$ (ddd, J = 9.8, 6.8, 5.3 Hz, 2H), 3.37 (td, J = 6.6, 1.2 Hz, 2H), 2.64 (ddd, J = 8.8, 6.2, $4.4 \mathrm{~Hz}, 1 \mathrm{H}$ ), 2.39 (dddd, J = 12.6, 8.9, 6.6, 2.6 Hz, 1H), $2.19-2.07(\mathrm{~m}, 1 \mathrm{H}), 2.05-1.87(\mathrm{~m}, 1 \mathrm{H}), 1.72-1.58(\mathrm{~m}, 1 \mathrm{H})$, $1.56-1.43(\mathrm{~m}, 2 \mathrm{H}), 1.38-1.24(\mathrm{~m}, 2 \mathrm{H}), 0.87(\mathrm{t}, \mathrm{J}=7.3 \mathrm{~Hz}, 3 \mathrm{H})$.

${ }^{13} \mathrm{C}-\mathrm{NMR}\left(126 \mathrm{MHz} ; \mathrm{CDCl}_{3}\right): \delta$ 179.78, 70.88, 68.29, 66.78, 36.94, 31.91, 30.44, 29.10, 19.49, 14.08.

LRMS (APCI) Calc. for $\left[\mathrm{C}_{10} \mathrm{H}_{18} \mathrm{O}_{3}\right]^{+}=186.13$, Found $=186.10$

HRMS (ESI) Calc. for $\left[\mathrm{C}_{10} \mathrm{H}_{18} \mathrm{O}_{3}+\mathrm{Na}\right]^{+}=209.1148$, Found $=209.1150$

$\mathbf{R}_{\mathbf{f}}=0.3$ in $15 \%$ ethyl acetate/hexanes

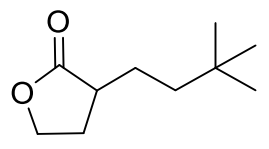

52b was synthesized via the general alkylation procedure in ( $13 \mathrm{mg}, 30 \%$ yield) as a white solid. 
Analytical data for $\mathbf{5 2 b}$

${ }^{1} \mathrm{H}-\mathrm{NMR}\left(300 \mathrm{MHz} ; \mathrm{CDCl}_{3}\right): \delta 4.31$ (td, J = 8.7, $\left.2.6 \mathrm{~Hz}, 1 \mathrm{H}\right), 4.14$ (ddd, J = 12.6, 9.3, $\left.6.4 \mathrm{~Hz}, 1 \mathrm{H}\right), 2.53$ $-2.25(\mathrm{~m}, 2 \mathrm{H}), 1.97-1.77(\mathrm{~m}, 2 \mathrm{H}), 1.45-1.10(\mathrm{~m}, 3 \mathrm{H}), 0.86(\mathrm{~s}, 9 \mathrm{H})$.

${ }^{13}$ C-NMR (126 MHz; $\left.\mathrm{CDCl}_{3}\right): \delta 179.76,66.69,41.86,39.99,30.42,29.42,28.83,25.79$

LRMS (APCI) Calc. for $\left[\mathrm{C}_{10} \mathrm{H}_{18} \mathrm{O}_{2}\right]^{+}=170.13$, Found $=170.10$

HRMS (ESI) Calc. for $\left[\mathrm{C}_{10} \mathrm{H}_{18} \mathrm{O}_{2}+\mathrm{Na}\right]^{+}=193.1199$, Found $=193.1197$

$\mathbf{R}_{\mathbf{f}}=0.4$ in $15 \%$ ethyl acetate/hexanes 


\section{H. References}

[1] a) Hird, A. W.; Hoveyda, A. H. J. J. Am. Chem. Soc. 2005, 127, 14988-14989.

[2] Green, S. A.; Vásquez-Céspedes, S.; Shenvi, R. A. J. Am. Chem. Soc. 2018, 140, 11317-11324.

[3] Gresser, M. J.; Wales, S. M.; Keller, P. A. Tetrahedron 2010, 66, 6965-6976.

[4] E. J. Corey, J. W. J. Suggs, J. Org. Chem. 1973, 38, 3223-3224.

[5] So, C. M.; Kume, S.; Hayashi, T. J. Am. Chem. Soc. 2013, 135, 10990 - 10993.

[6] Zhong, S.; Mondon, M.; Pilard, S.; Len, C. Tetrahedron 2008, 64, 7828-7836.

[7] Annand, J. R.; Bruno, P. A.; Mapp, A. K.; Schindler, C. S. Chem. Commun. 2015, 51, 8990-8993

[8] Enrich, P. M.; Tosin, M.; Nieuwenhuyzen, M.; Cronin, L.; Murphy, P. V. J. Org. Chem. 2002, 67, 3733-3741

[9] Przydacz, A.; Kowalczyk, R.; Albrecht, Ł. Org. Biomol. Chem. 2017, 15, 9566-9569.

[10] Dardonille, C.; Rinaldi, E.; Barrett, M. P.; Bru, R.; Gilbert, I. H.; Hanau, S. J. Med. Chem. 2004, 47, $3427-3437$.

[11] Berte-Verrando, S.; Neif, F.; Patois, C.; Savignac, P. Phosphorus, Sulfur, and Silicon 1995, 103, 91100.

[12] Hilton, S. T.; Motherwell, W. B.; Potier, P.; Pradet, C.; Selwood, D. L. Bioorg. Med. Chem. Lett. 2005, 15, 2239-2242.

[13] Benati, L.; Leardini, R.; Minozzi, M. Angew. Chem. Int. Ed. 2004, 43, 3598-3601. 
I. 1H, 13C, and 2-D NMR Spectra

${ }^{1} \mathrm{H}-\mathrm{NMR}$ (500 MHz; $\mathrm{CDCl}_{3}$ )<smiles>C=CCOC(=O)NCc1cccs1</smiles>

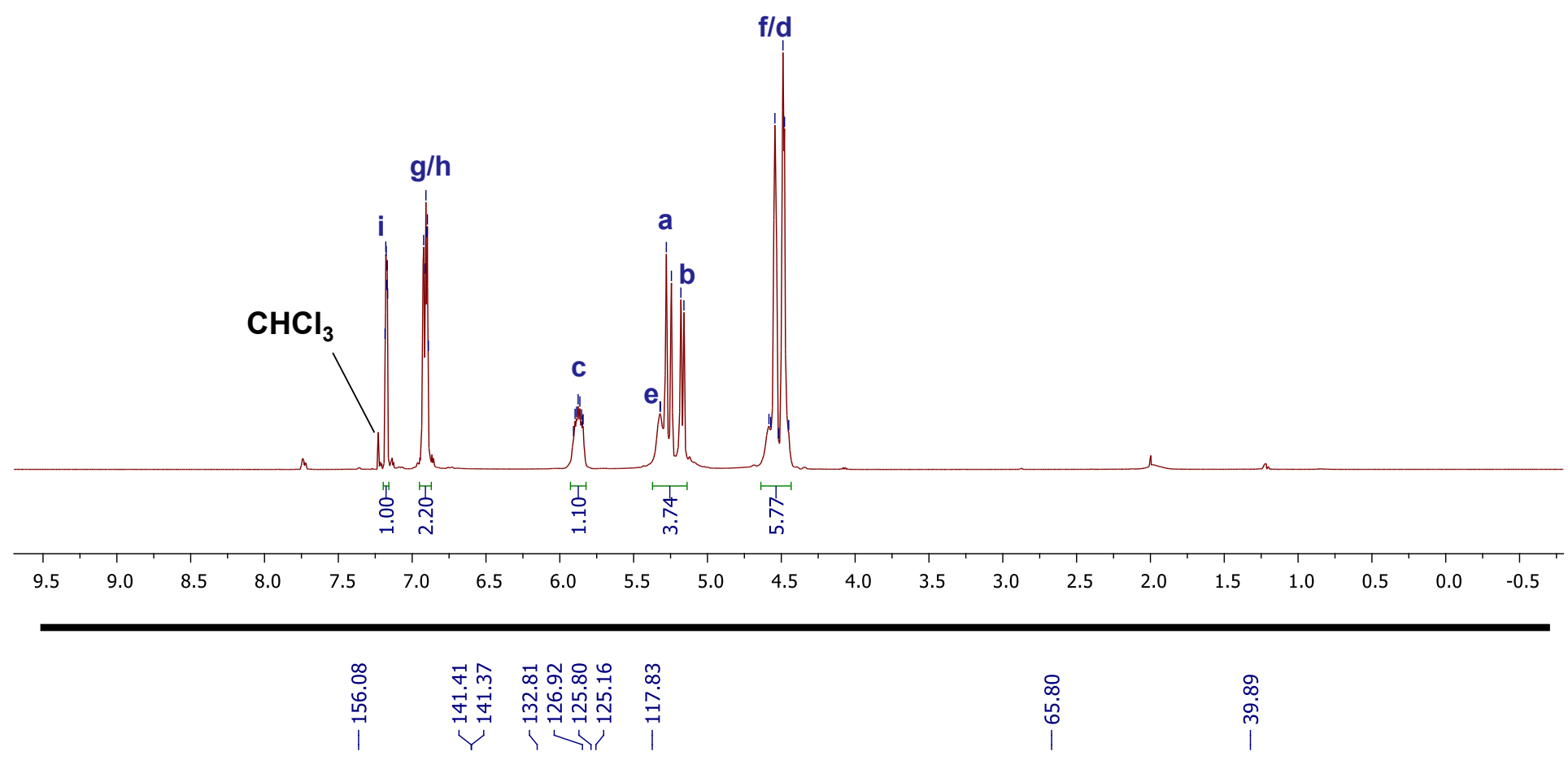

${ }^{13} \mathrm{C}-\mathrm{NMR}\left(126 \mathrm{MHz} ; \mathrm{CDCl}_{3}\right.$ )

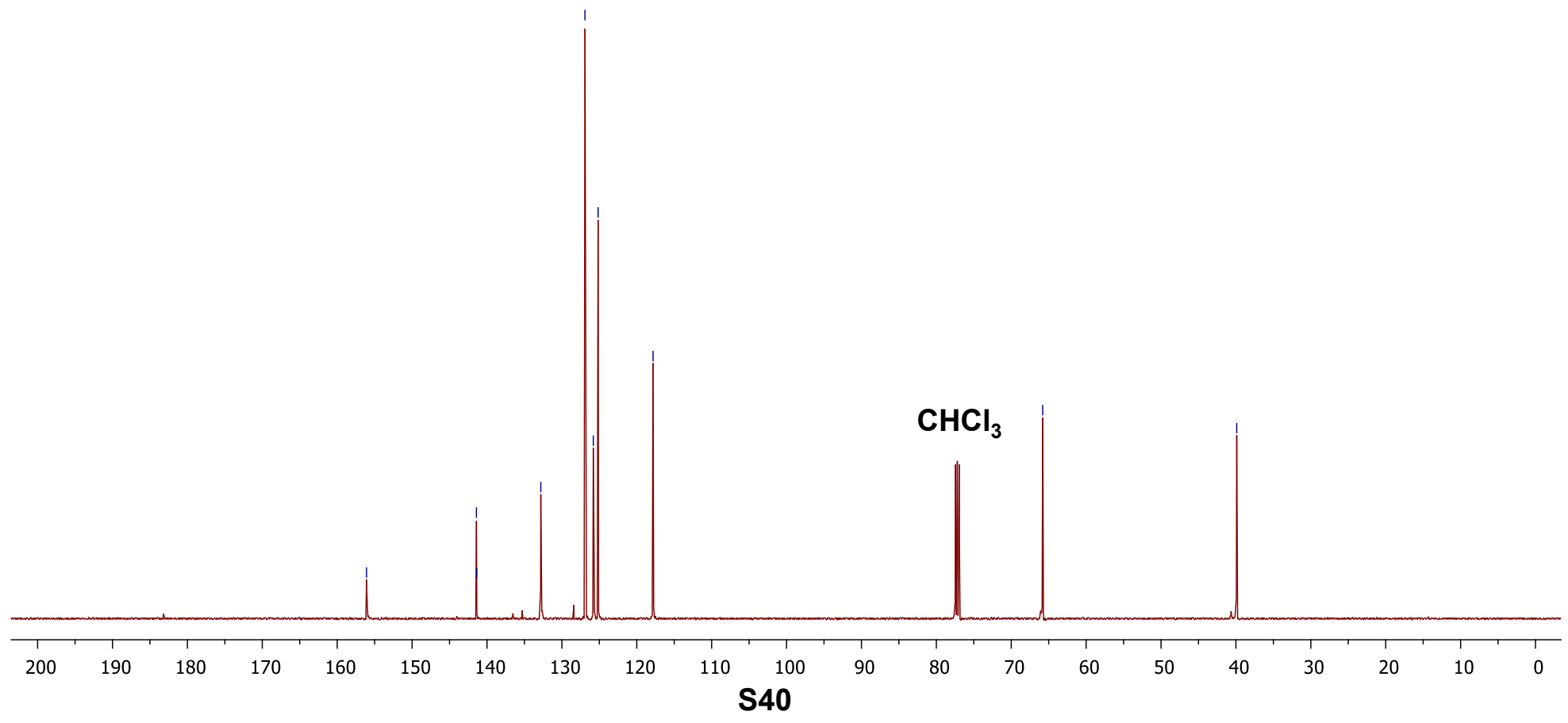


${ }^{1} \mathrm{H}-\mathrm{NMR}\left(500 \mathrm{MHz} ; \mathrm{CDCl}_{3}\right.$ )
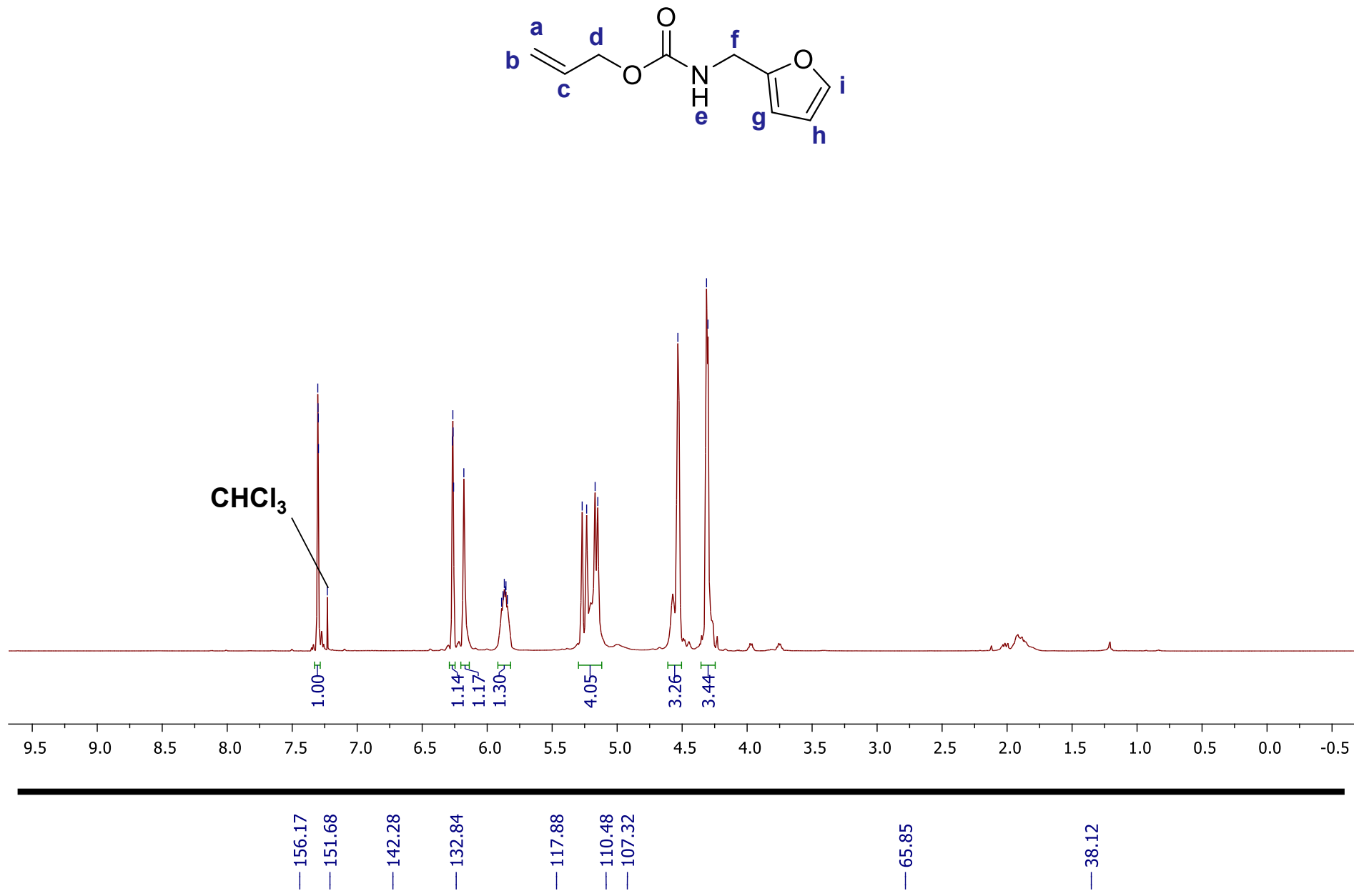

${ }^{13} \mathrm{C}-\mathrm{NMR}\left(126 \mathrm{MHz} ; \mathrm{CDCl}_{3}\right)$

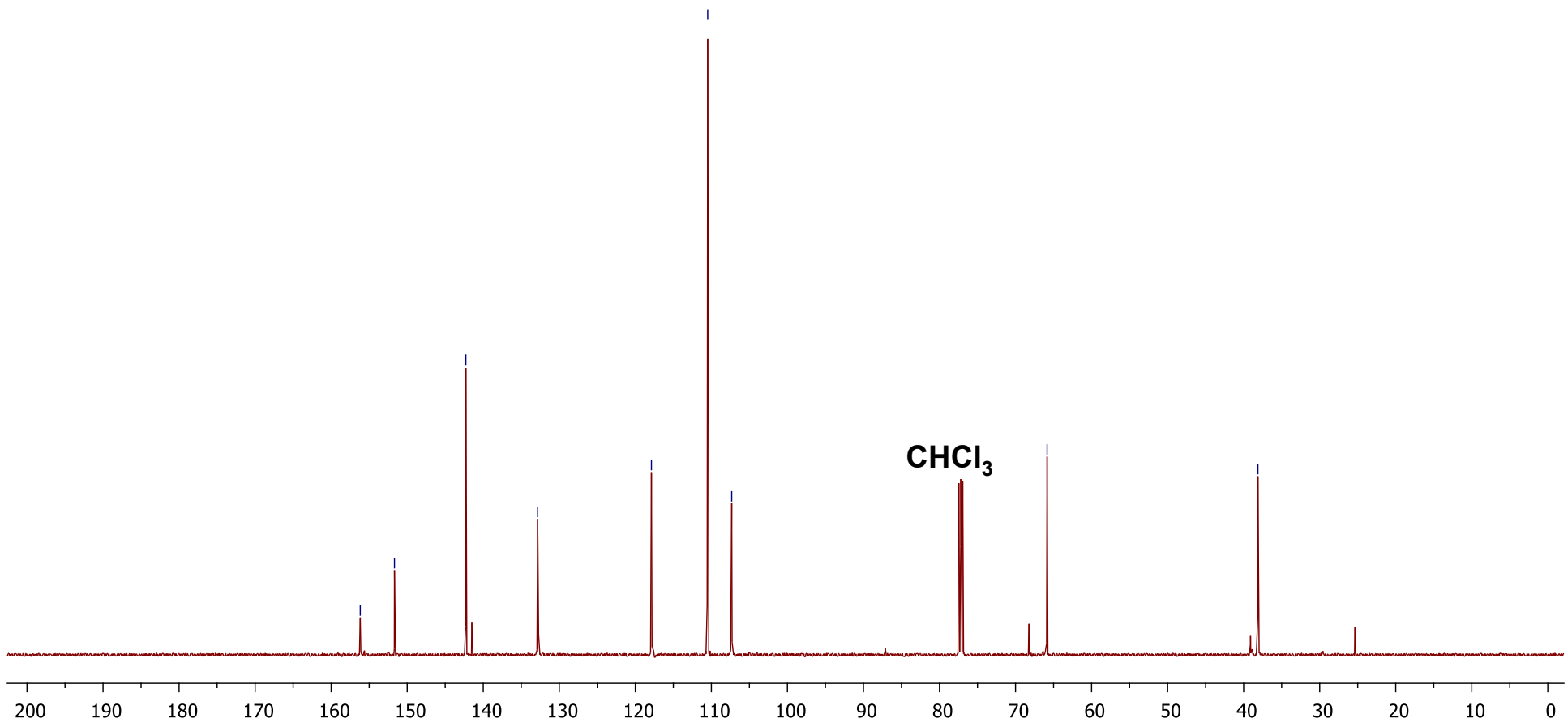


${ }^{1} \mathrm{H}-\mathrm{NMR}\left(500 \mathrm{MHz} ; \mathrm{CDCl}_{3}\right.$ )

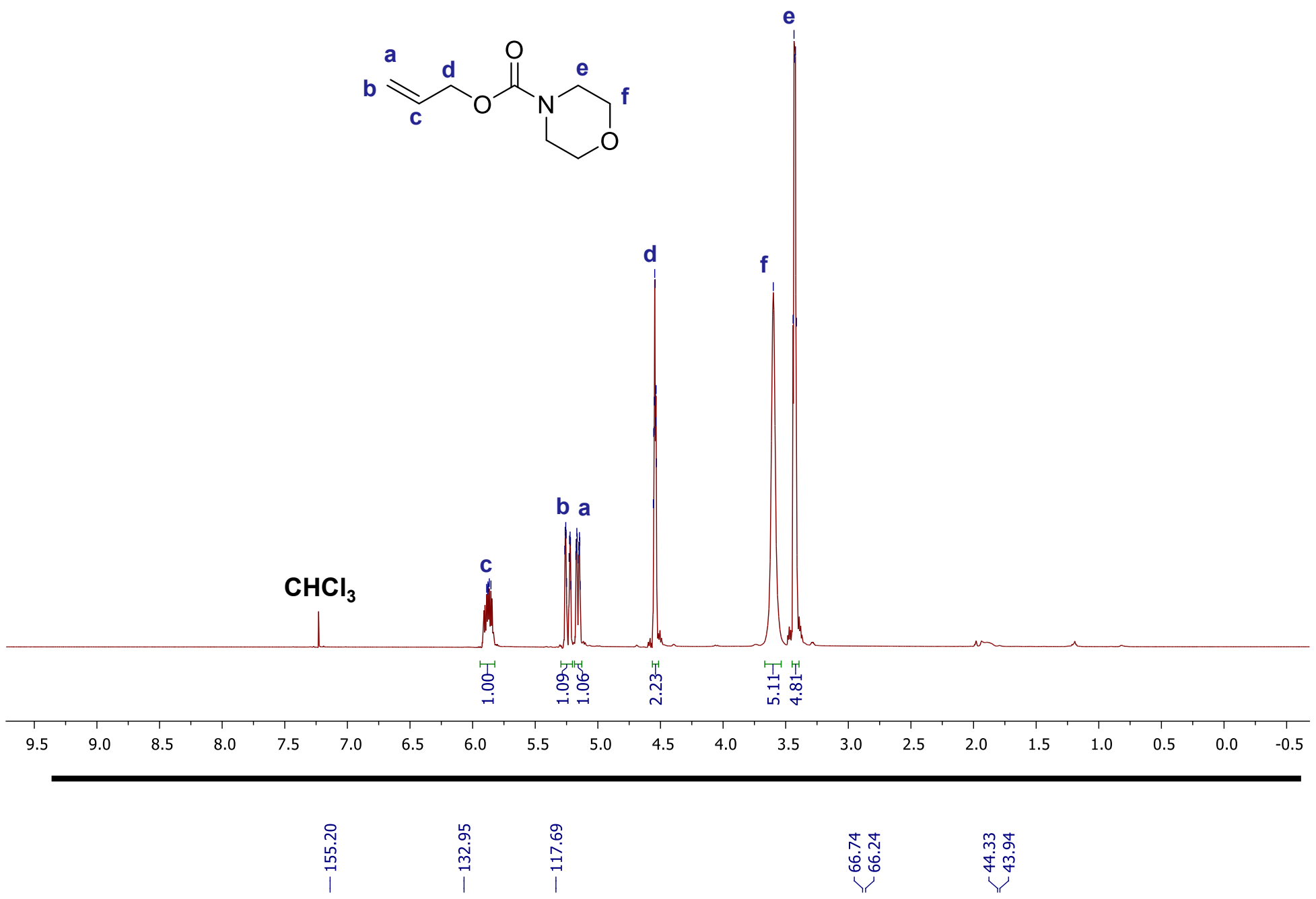

${ }^{13} \mathrm{C}-\mathrm{NMR}\left(126 \mathrm{MHz} ; \mathrm{CDCl}_{3}\right)$

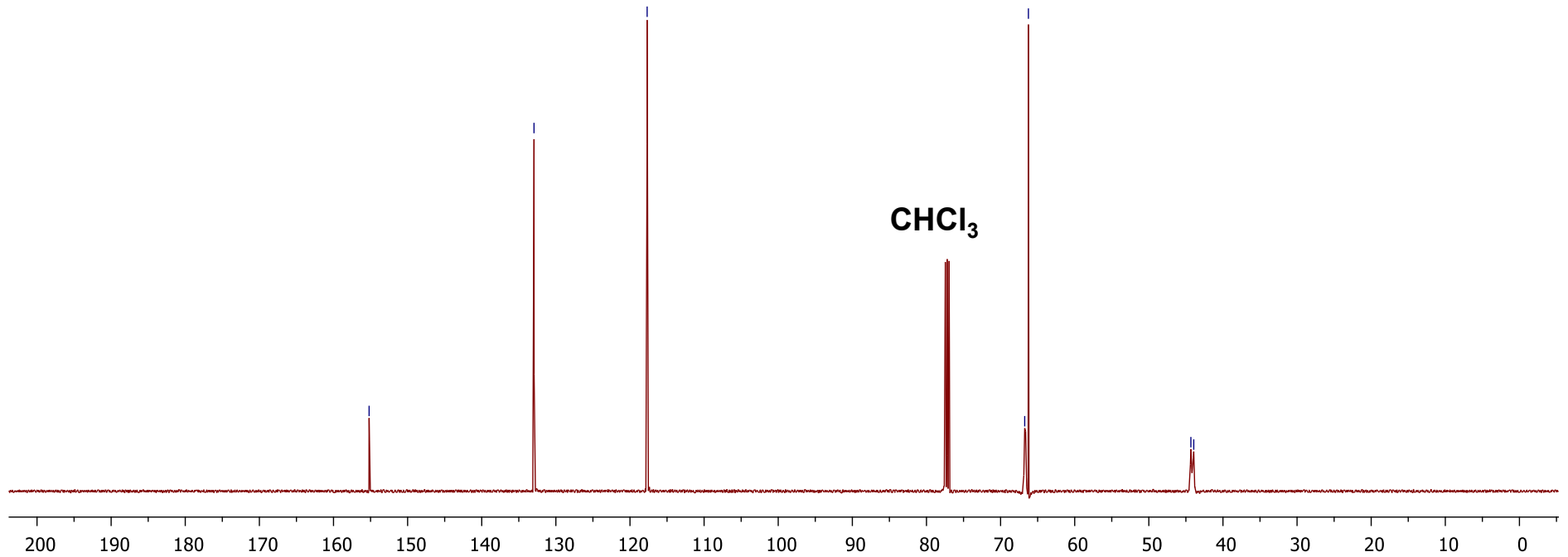


${ }^{1} \mathrm{H}-\mathrm{NMR}\left(500 \mathrm{MHz} ; \mathrm{CDCl}_{3}\right.$ )

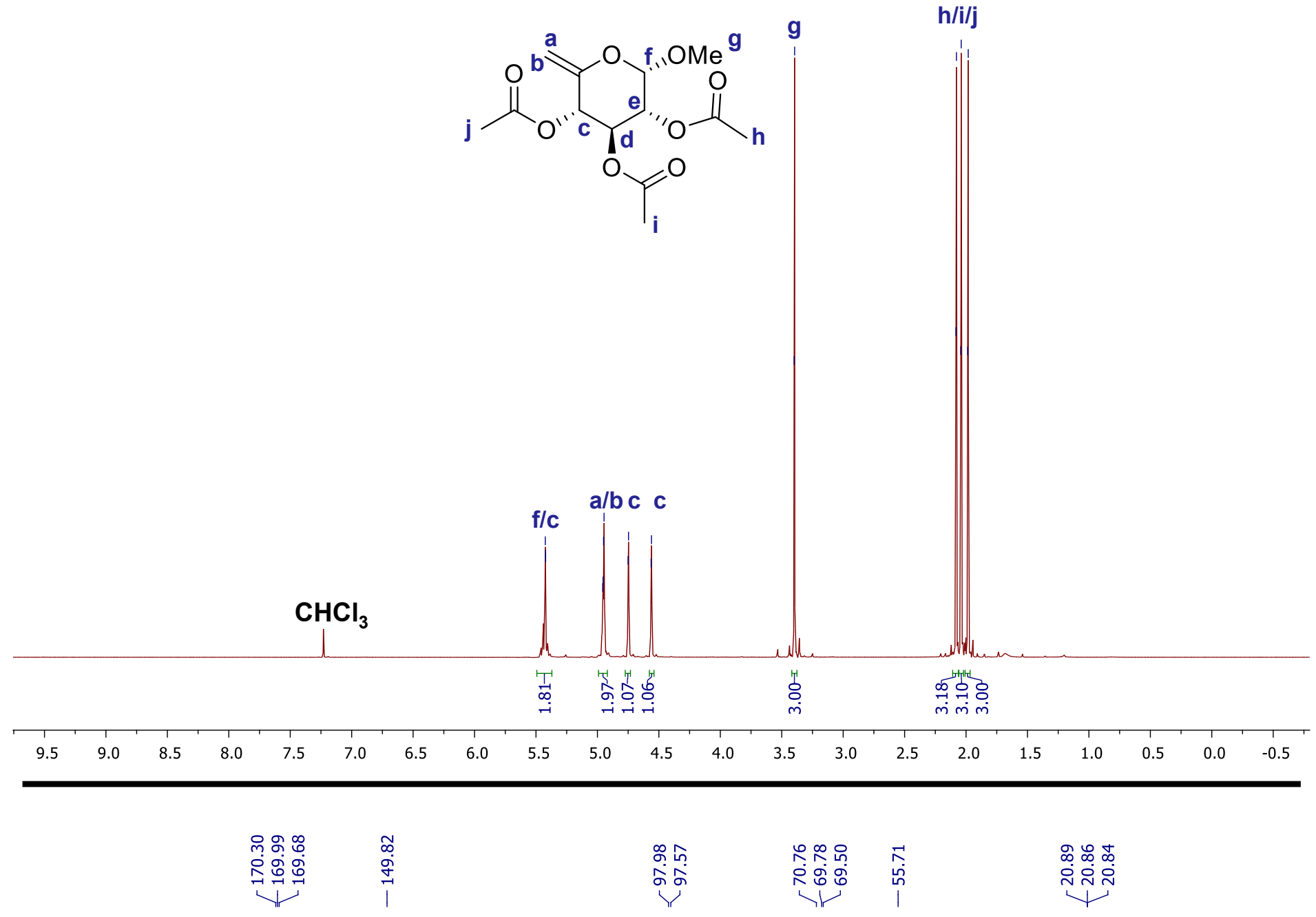

${ }^{13} \mathrm{C}-\mathrm{NMR}\left(126 \mathrm{MHz} ; \mathrm{CDCl}_{3}\right.$ )

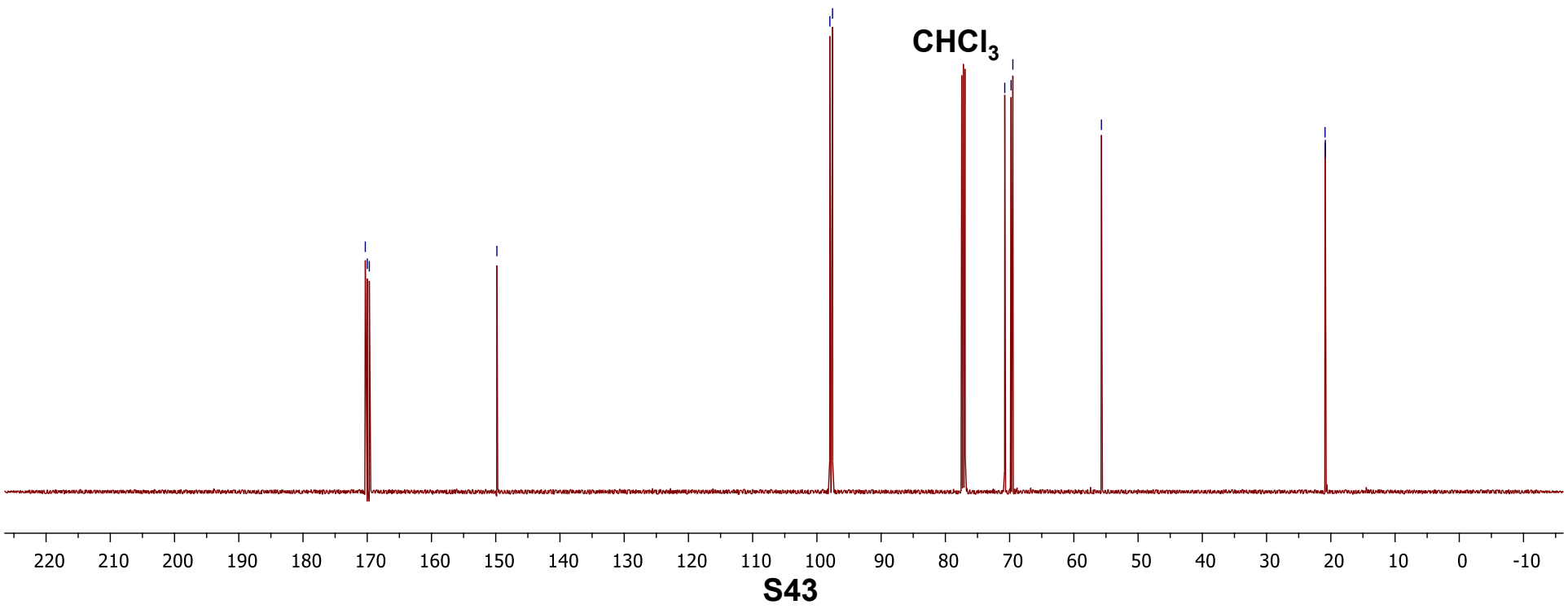


${ }^{1} \mathrm{H}-\mathrm{NMR}\left(300 \mathrm{MHz} ; \mathrm{CDCl}_{3}\right.$ )<smiles>O=C(CS)c1ccc(F)cc1</smiles>

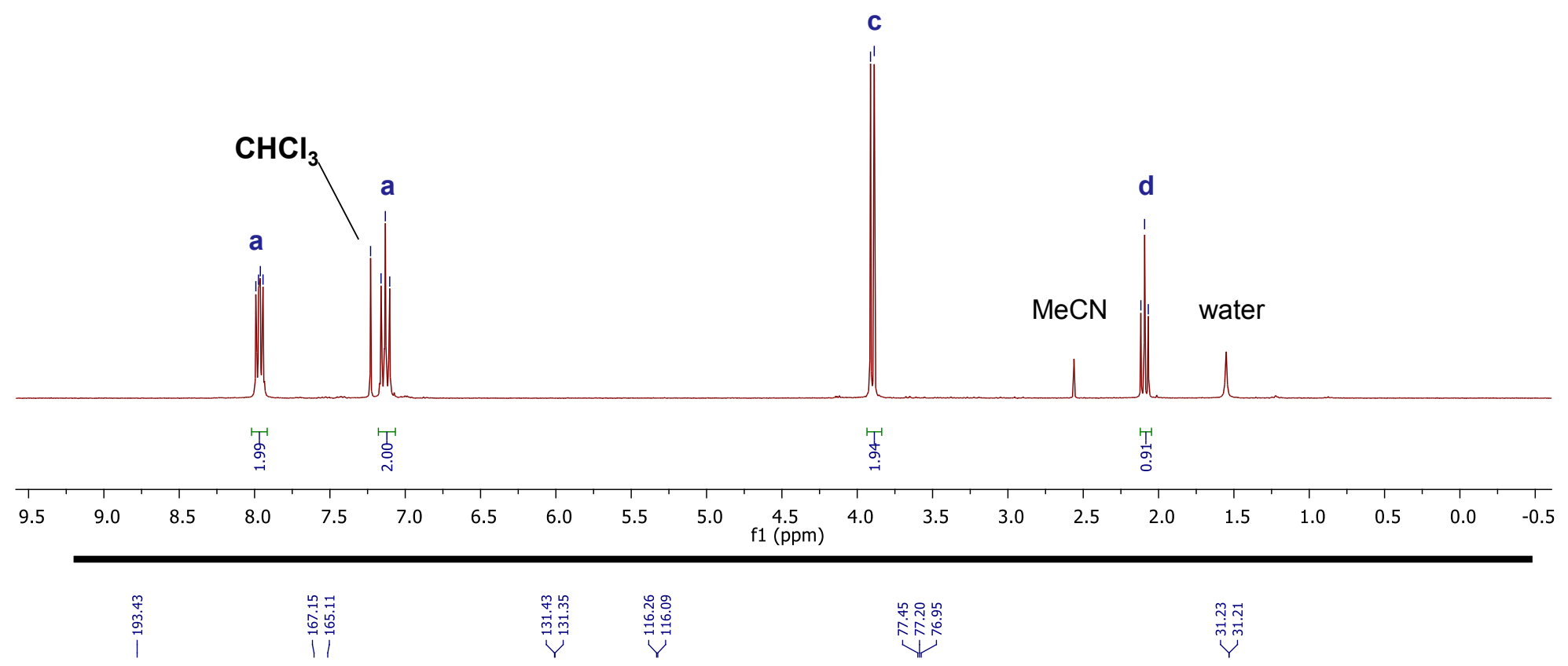

${ }^{13} \mathrm{C}-\mathrm{NMR}\left(126 \mathrm{MHz} ; \mathrm{CDCl}_{3}\right.$ )
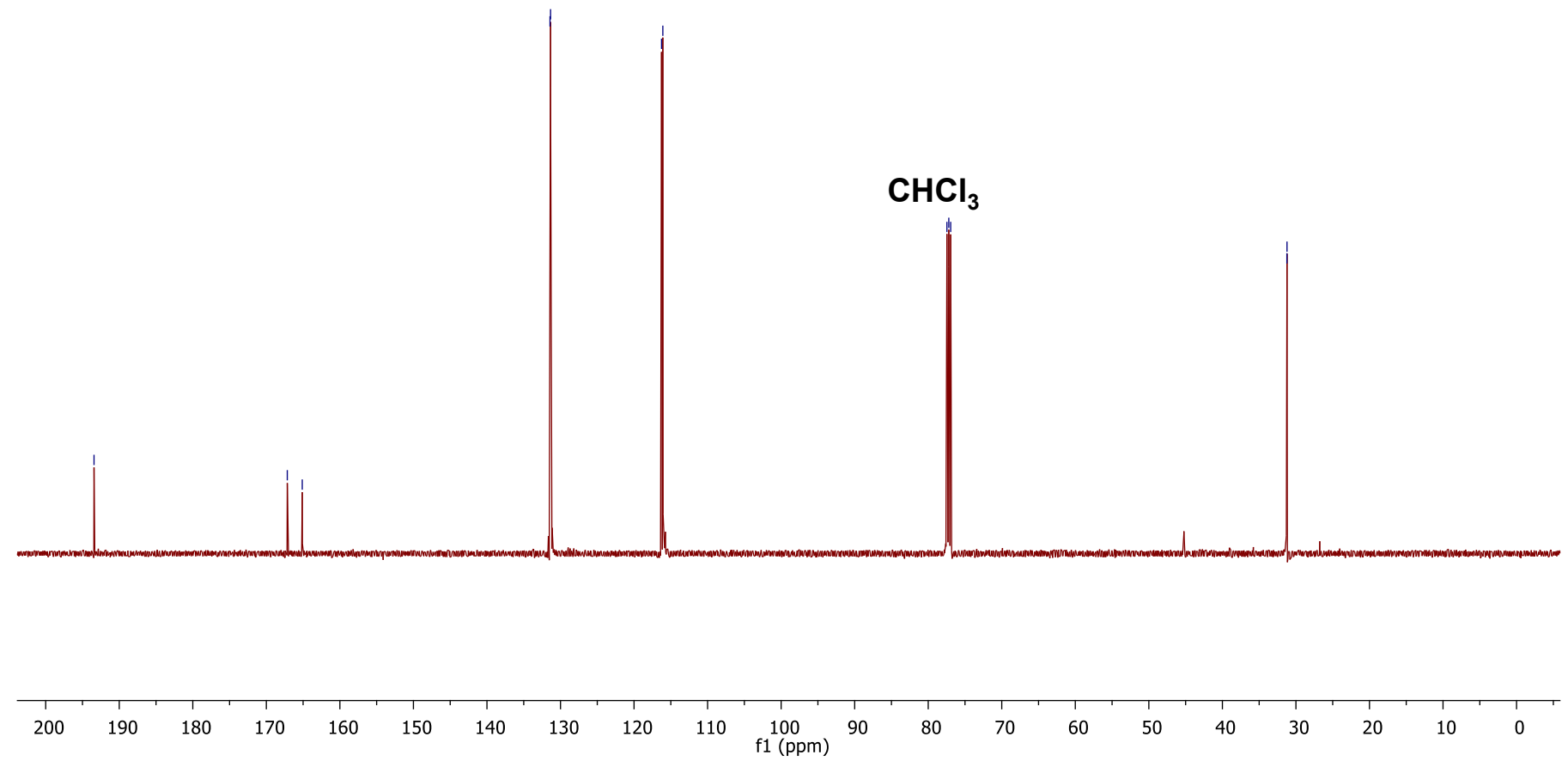
${ }^{1} \mathrm{H}-\mathrm{NMR}\left(300 \mathrm{MHz} ; \mathrm{CDCl}_{3}\right)$ i
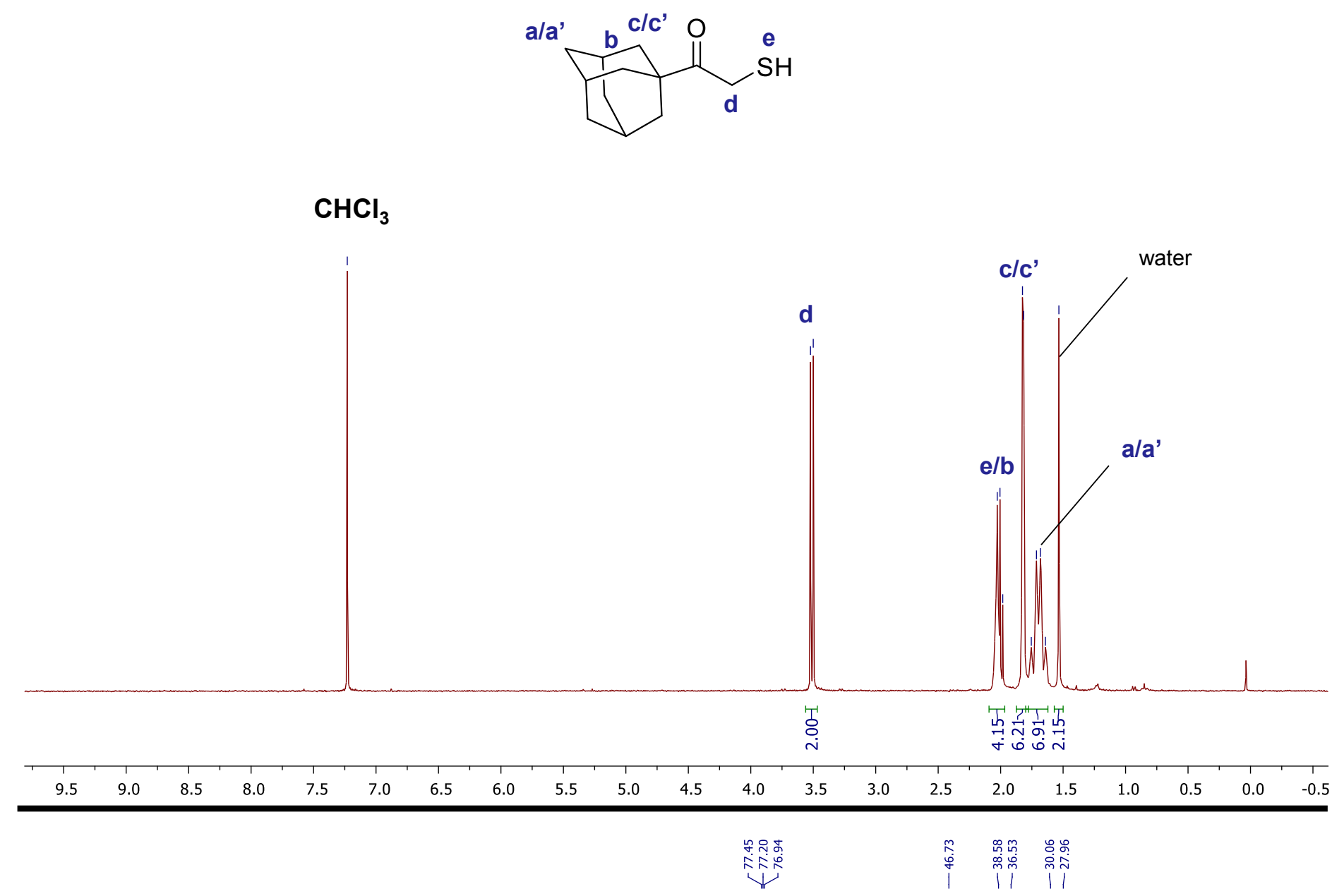

${ }^{13} \mathrm{C}-\mathrm{NMR}$ (126 MHz; $\mathrm{CDCl}_{3}$ )

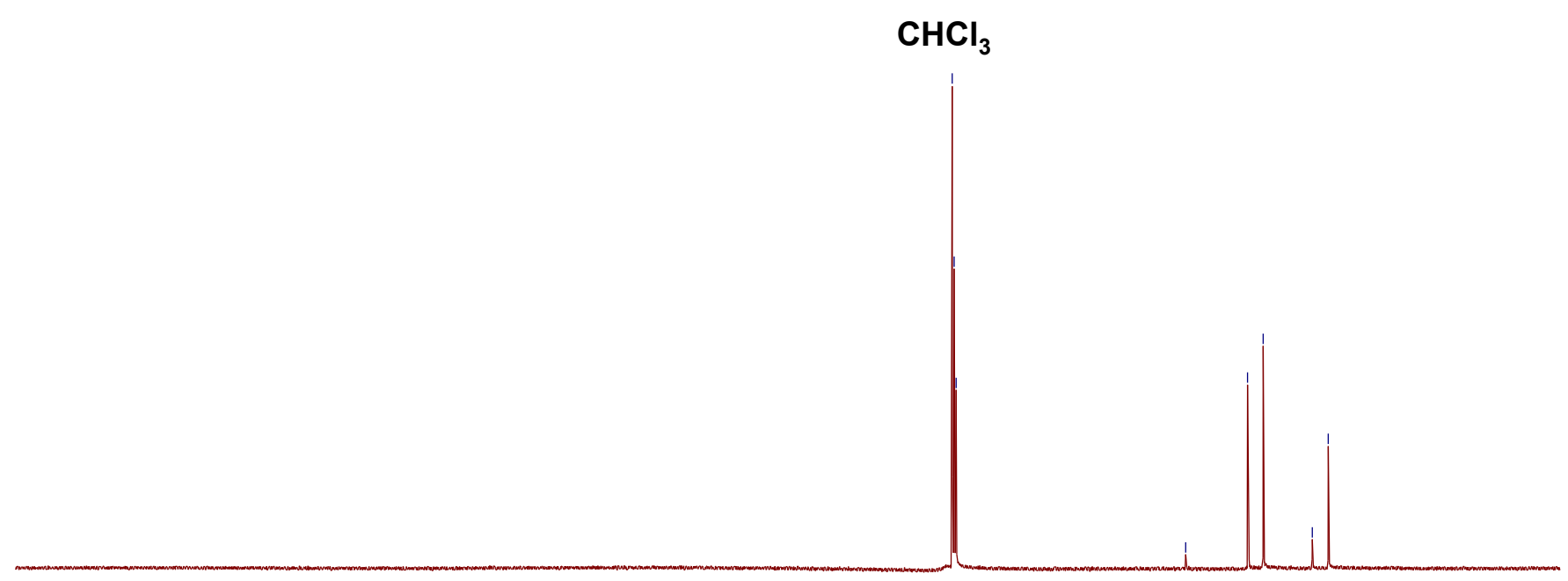

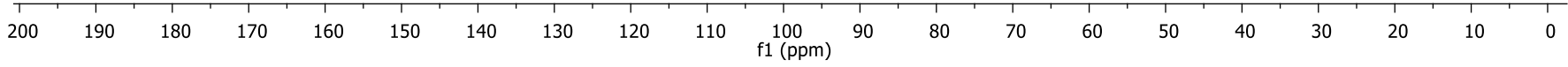


${ }^{1} \mathrm{H}-\mathrm{NMR}$ (300 MHz; $\mathrm{CDCl}_{3}$ )

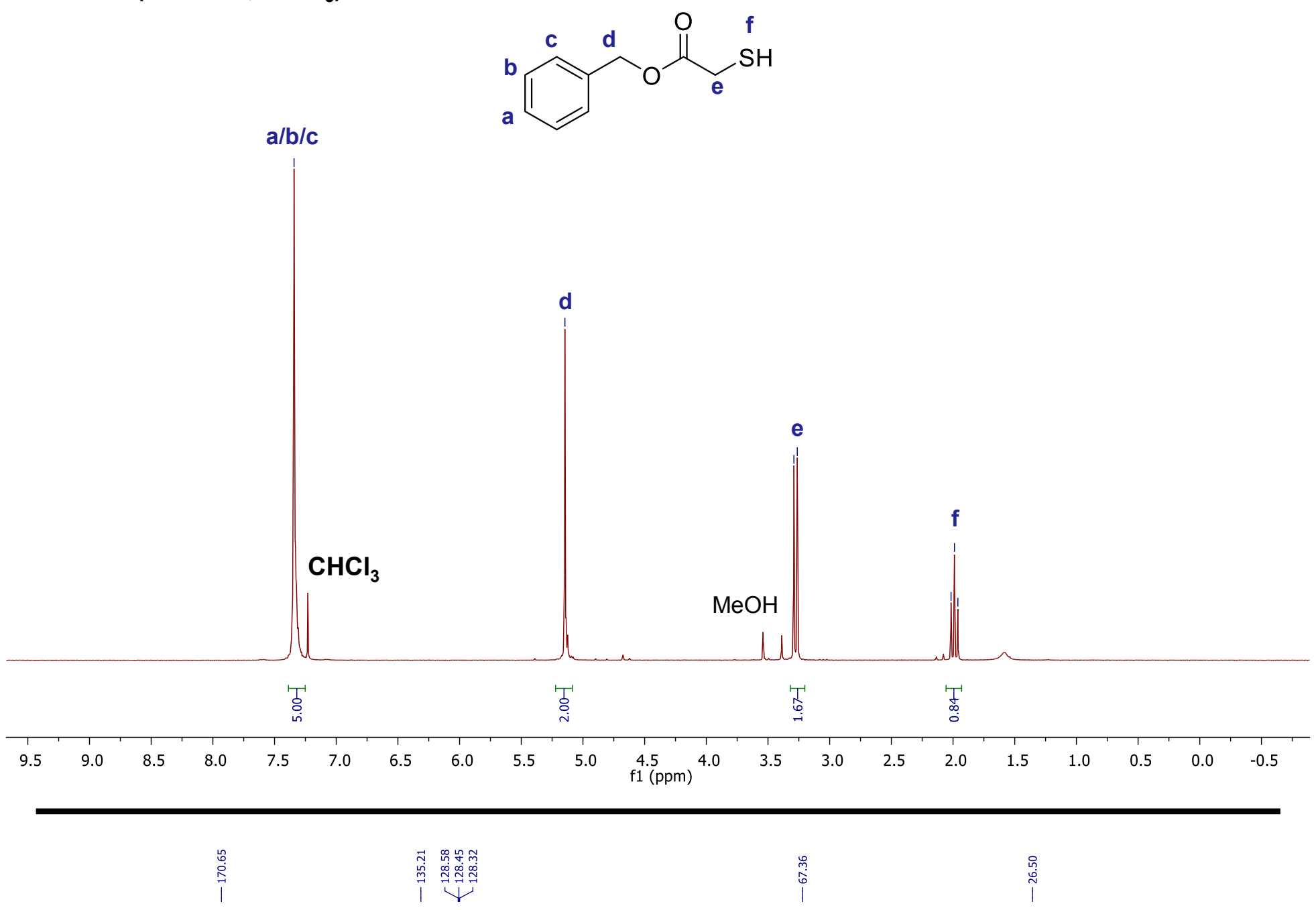

${ }^{13} \mathrm{C}-\mathrm{NMR}$ (126 MHz; $\mathrm{CDCl}_{3}$ )

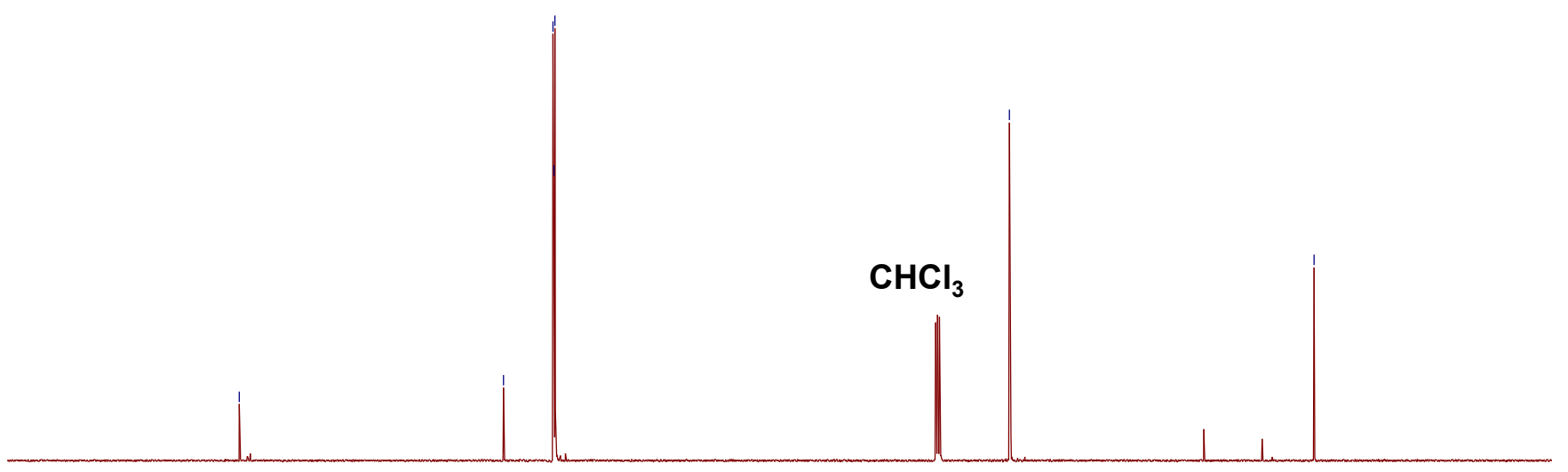

\begin{tabular}{|c|c|c|c|c|c|c|c|c|c|c|c|c|c|c|c|c|c|c|c|c|}
\hline$T$ & 1 & 1 & $T$ & $T$ & 1 & $T$ & $T$ & $T$ & 1 & 1 & 1 & $T$ & 1 & 1 & $T$ & $T$ & $T$ & $T$ & $T$ & 1 \\
\hline 200 & 190 & 180 & 170 & 160 & 150 & 140 & 130 & 120 & 110 & $\begin{array}{c}100 \\
\mathrm{f} 1(\mathrm{ppm})\end{array}$ & 90 & 80 & 70 & 60 & 50 & 40 & 30 & 20 & 10 & 0 \\
\hline
\end{tabular}


${ }^{1} \mathrm{H}-\mathrm{NMR}$ (300 MHz; $\mathrm{CDCl}_{3}$ )<smiles>CSP(=O)(CS)OC[CH]C(F)(F)F</smiles><smiles>CC(=O)SCP(=O)(OCC(F)(F)F)OCC(F)(F)F</smiles>

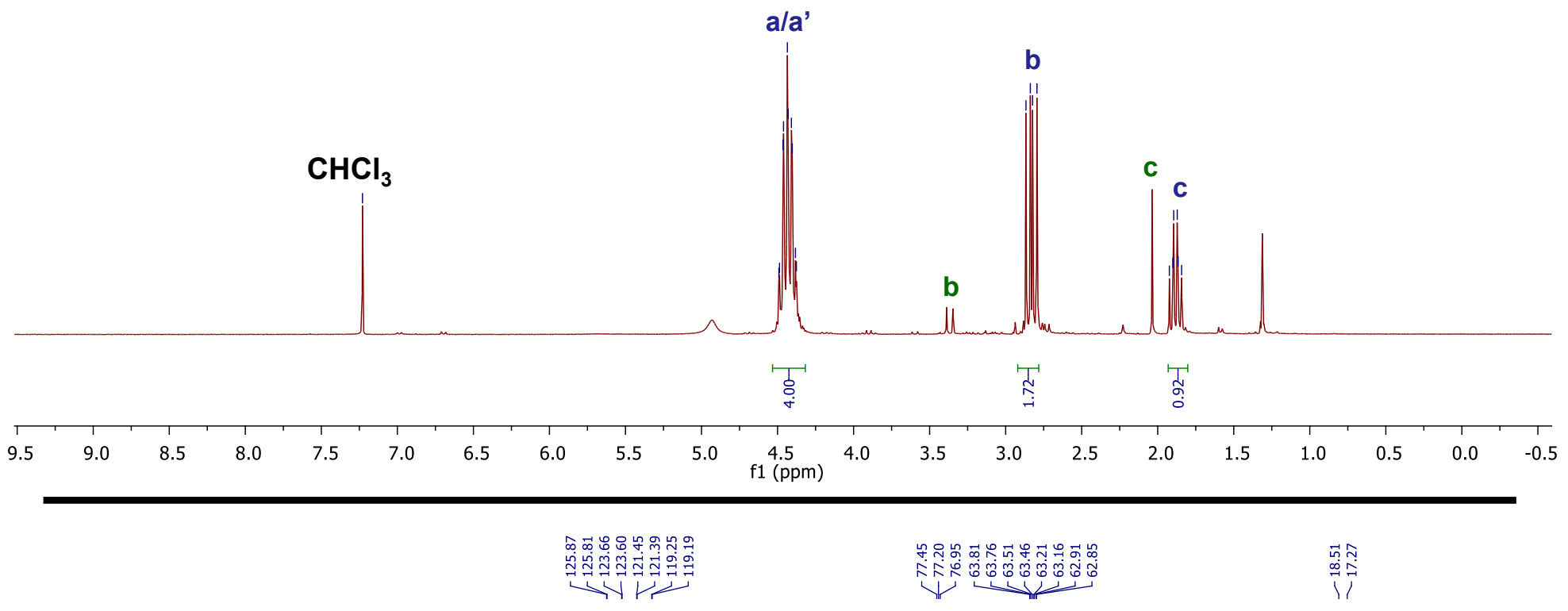

${ }^{13} \mathrm{C}-\mathrm{NMR}$ (126 MHz; $\mathrm{CDCl}_{3}$ )

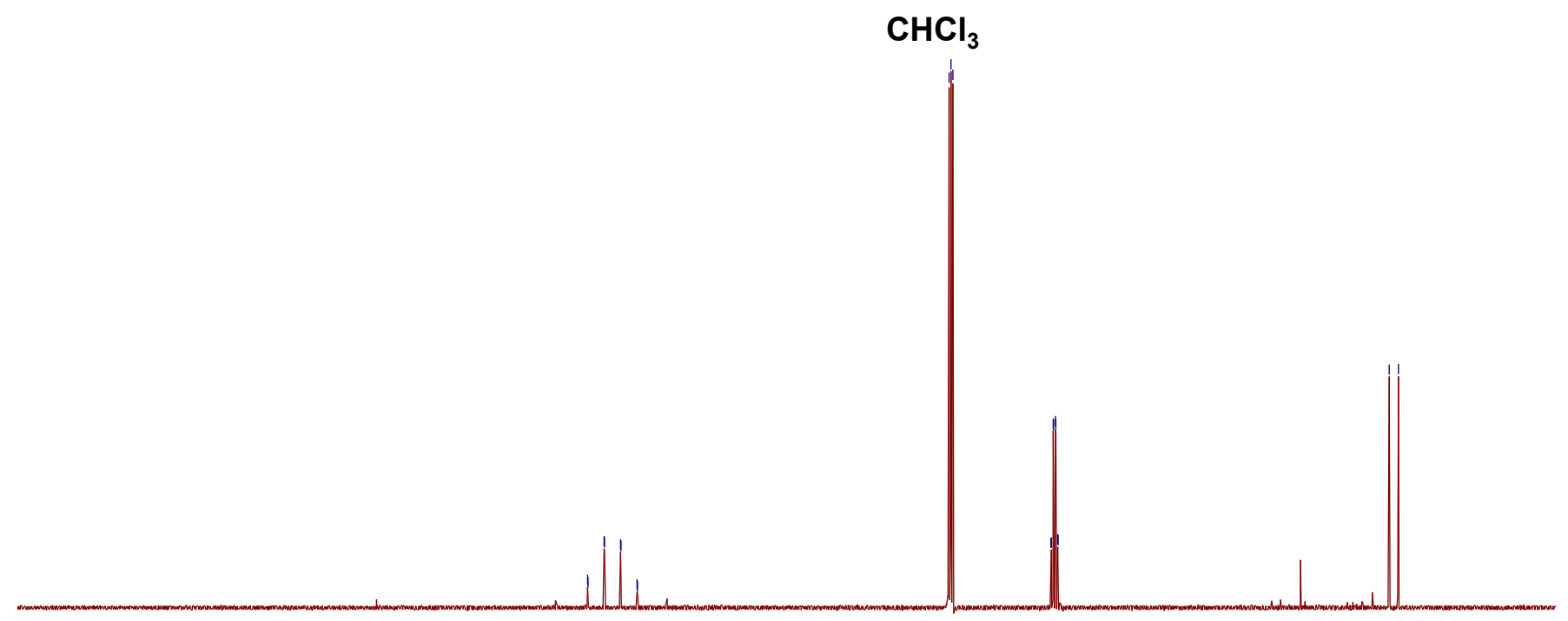

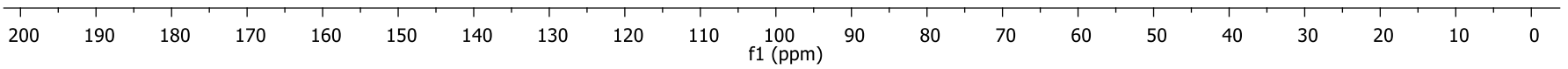


${ }^{19}$ F NMR (282 MHz, CDCl3)<smiles>O=P(CS)(OCC(F)(F)F)OCC(F)(F)F</smiles>
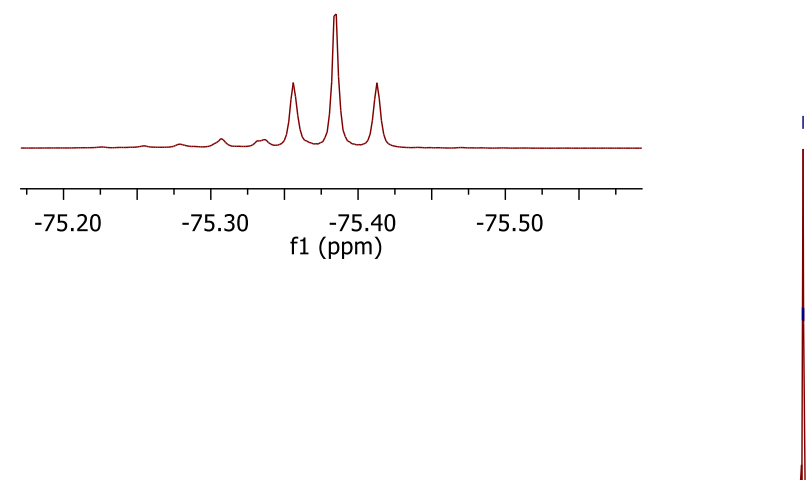

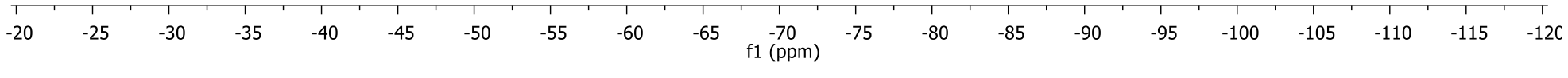

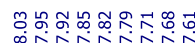

iñañana

31P NMR (212 MHz, CDCl3)

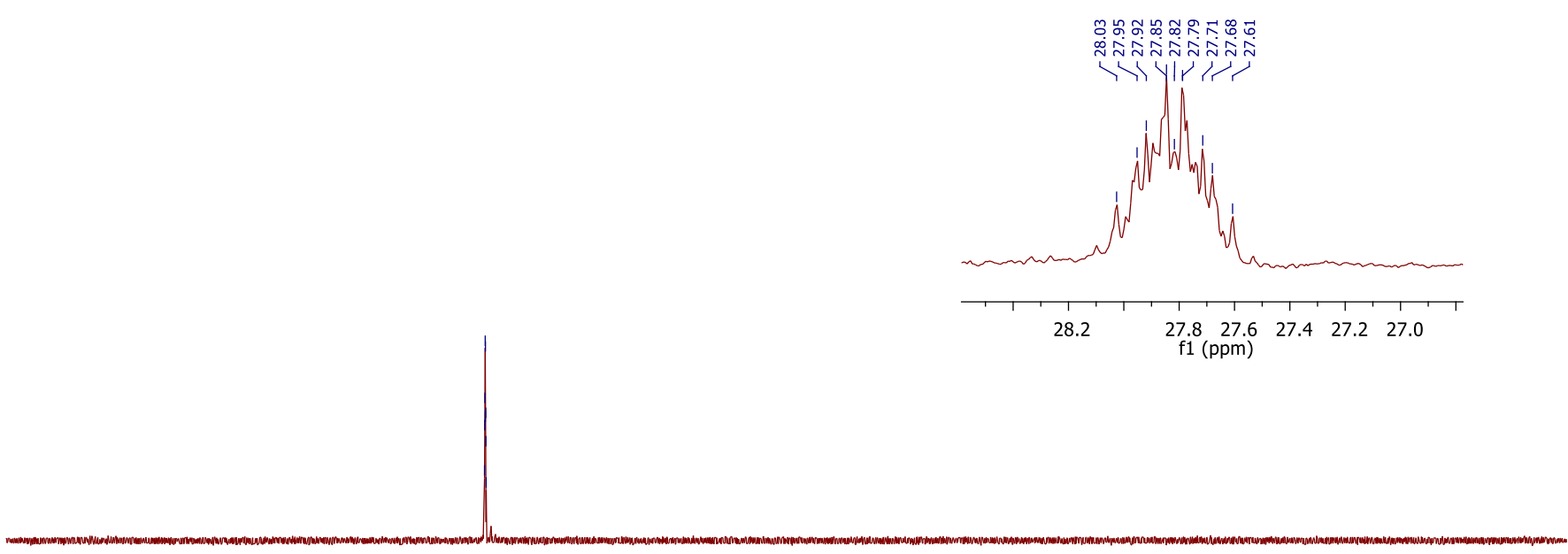

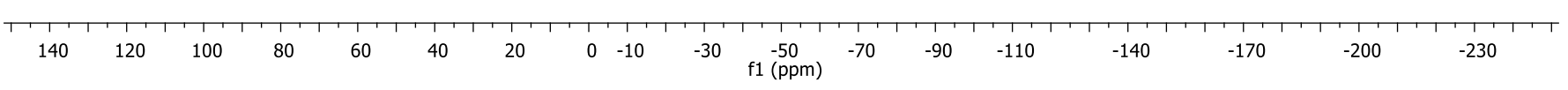


${ }^{1} \mathrm{H}-\mathrm{NMR}\left(300 \mathrm{MHz} ; \mathrm{CDCl}_{3}\right)$

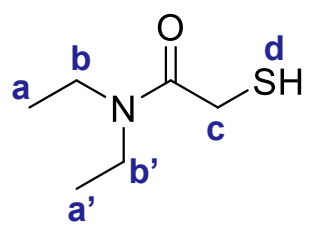

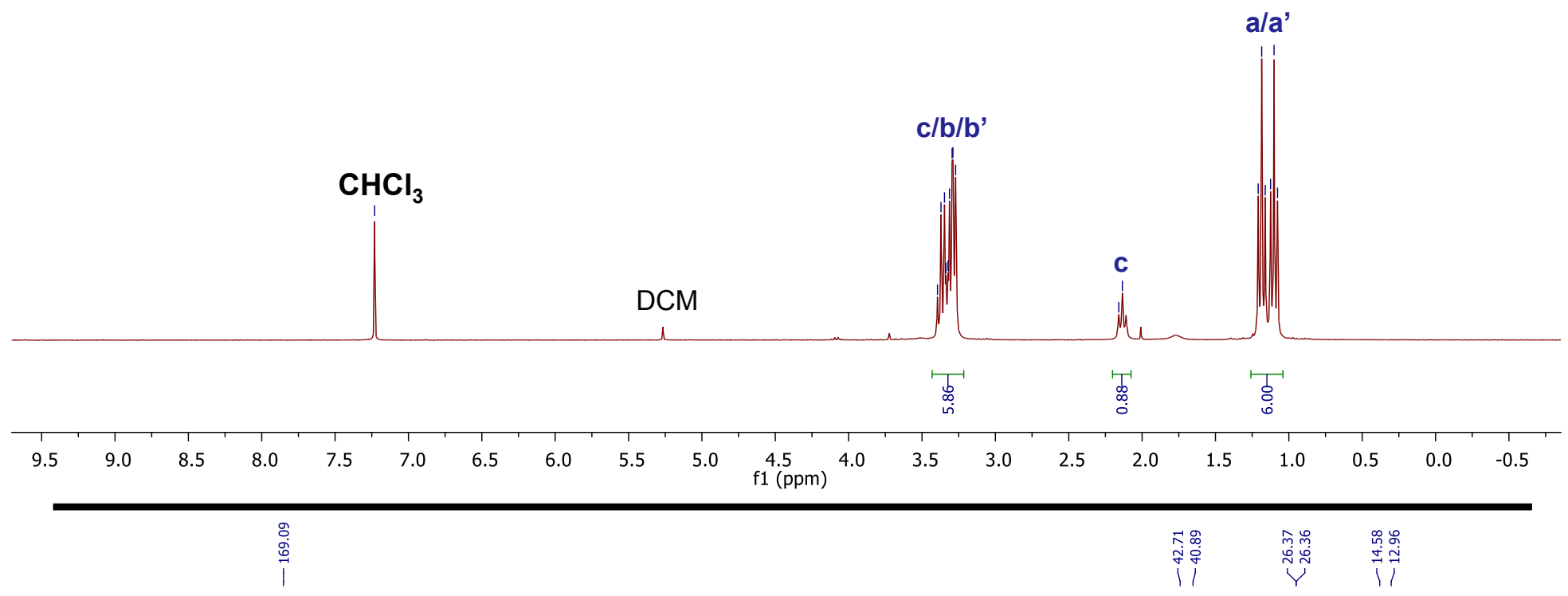

${ }^{13} \mathrm{C}-\mathrm{NMR}\left(126 \mathrm{MHz}^{\mathrm{CDCl}}{ }_{3}\right)$

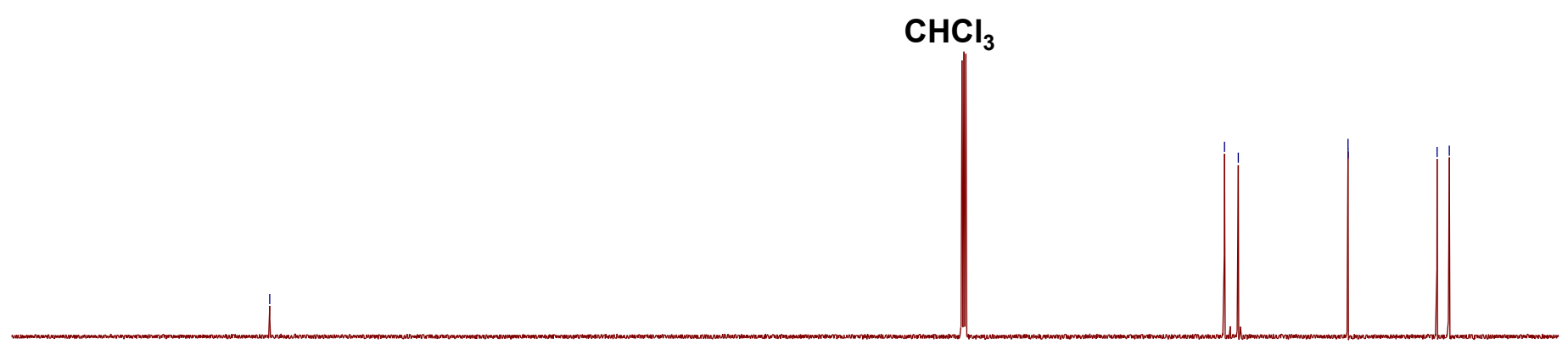

\begin{tabular}{llllllllllllllllllllll}
\hline 200 & 190 & 180 & 170 & 160 & 150 & 140 & 130 & 120 & 110 & $\begin{array}{c}100 \\
\mathrm{f}(\mathrm{ppm})\end{array}$ & 90 & 80 & 70 & 60 & 50 & 40 & 30 & 20 & 10 & 0
\end{tabular}


${ }^{1} \mathrm{H}-\mathrm{NMR}\left(300 \mathrm{MHz} ; \mathrm{CDCl}_{3}\right.$ )

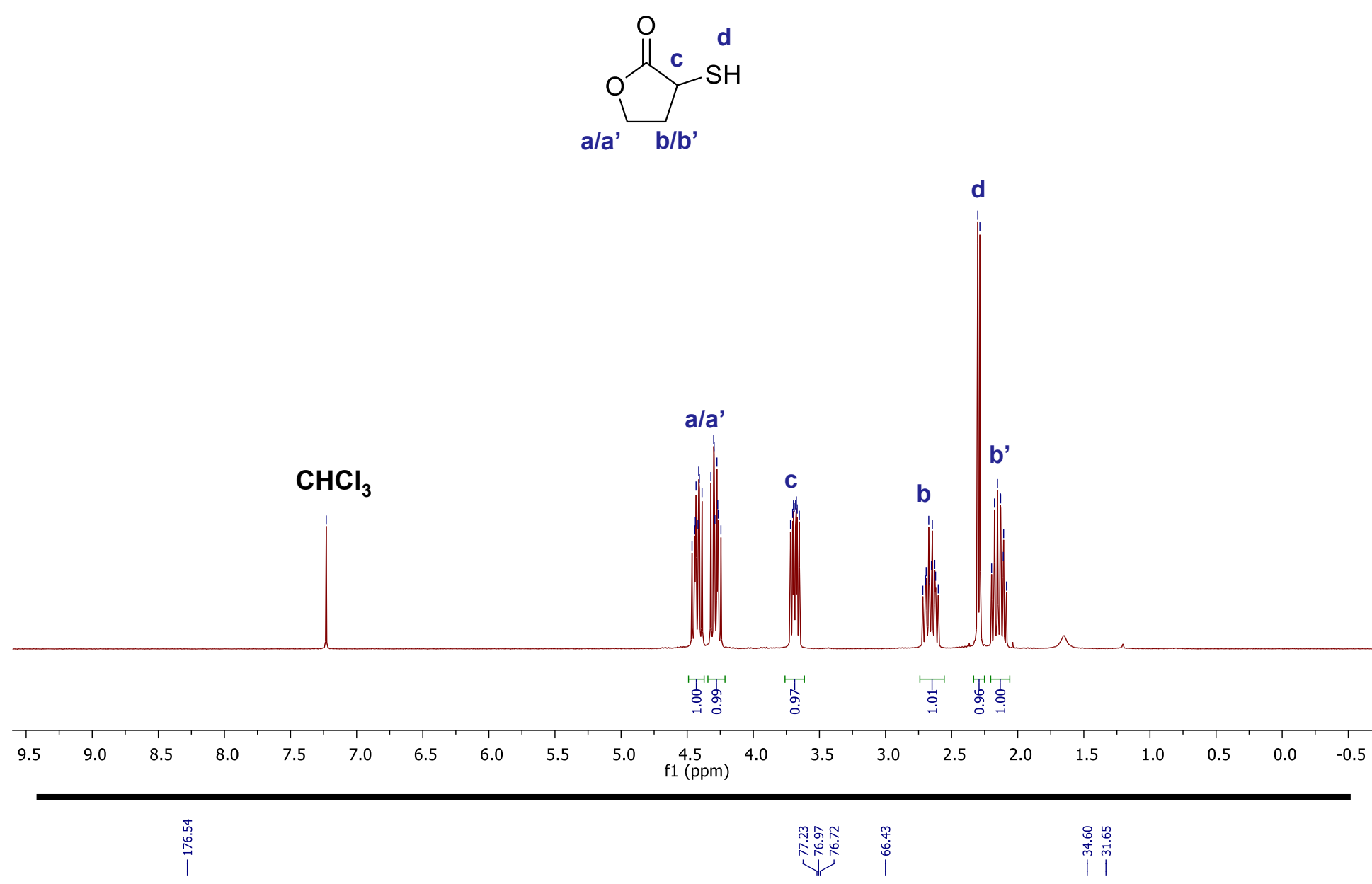

${ }^{13} \mathrm{C}-\mathrm{NMR}\left(126 \mathrm{MHz} ; \mathrm{CDCl}_{3}\right.$ )

$\mathrm{CHCl}_{3}$

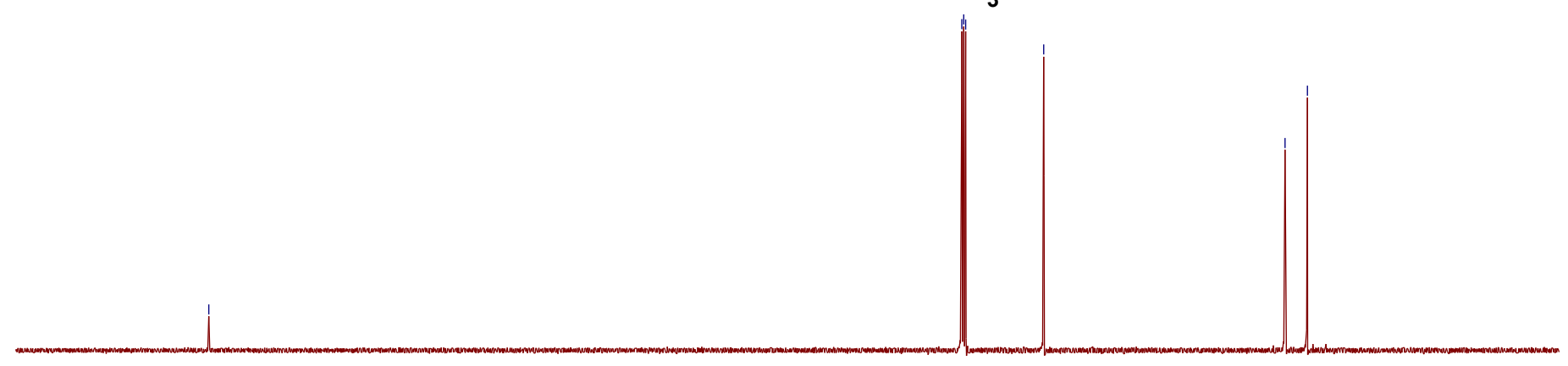

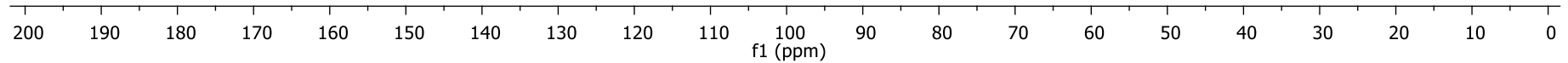


${ }^{1} \mathrm{H}-\mathrm{NMR}\left(300 \mathrm{MHz} ; \mathrm{CDCl}_{3}\right.$ )

a<smiles>[Y]C(C)CCOC(I)CCC(=O)c1ccc(OC)cc1</smiles>

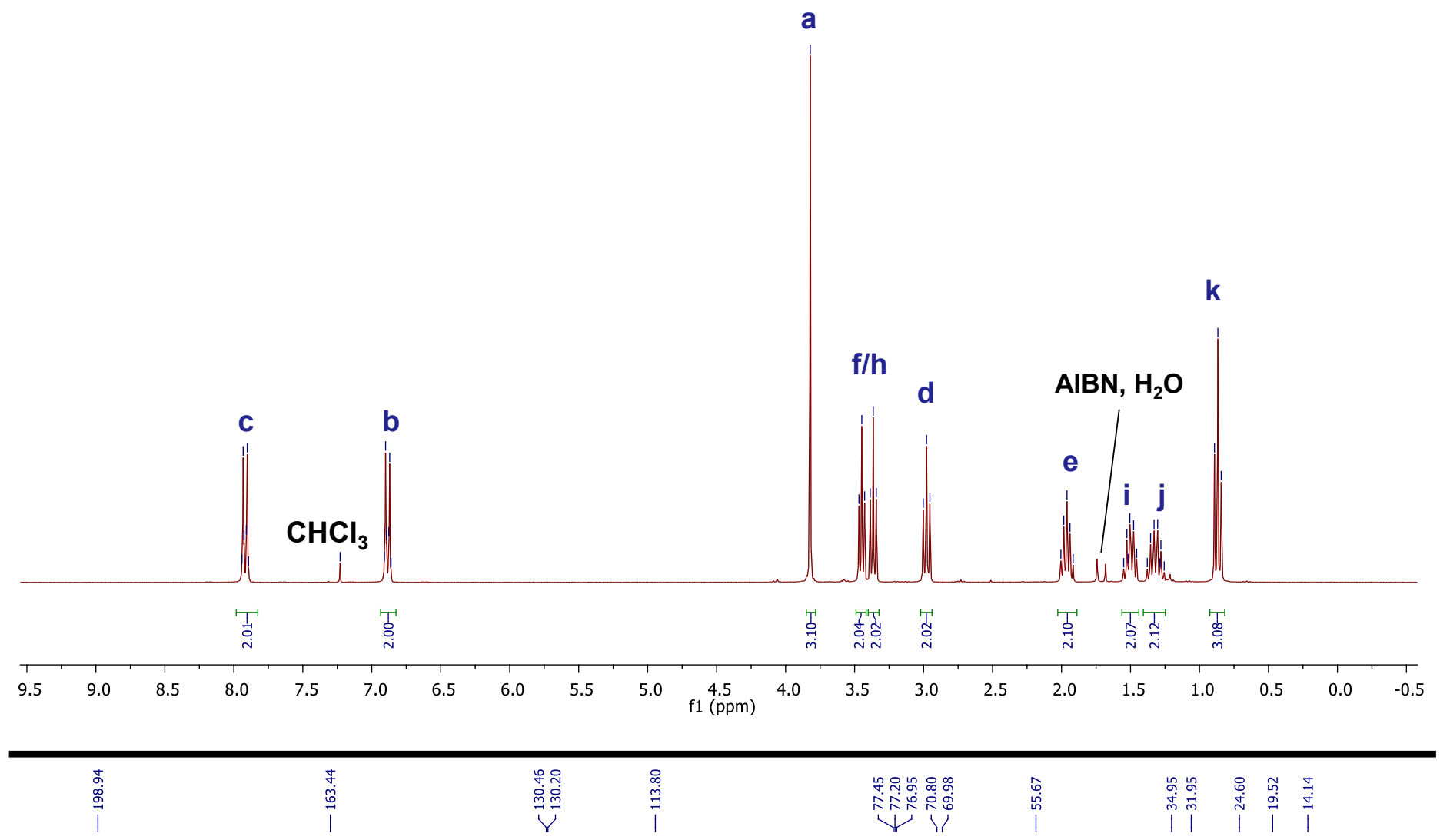

${ }^{13} \mathrm{C}-\mathrm{NMR}\left(126 \mathrm{MHz} ; \mathrm{CDCl}_{3}\right.$ )

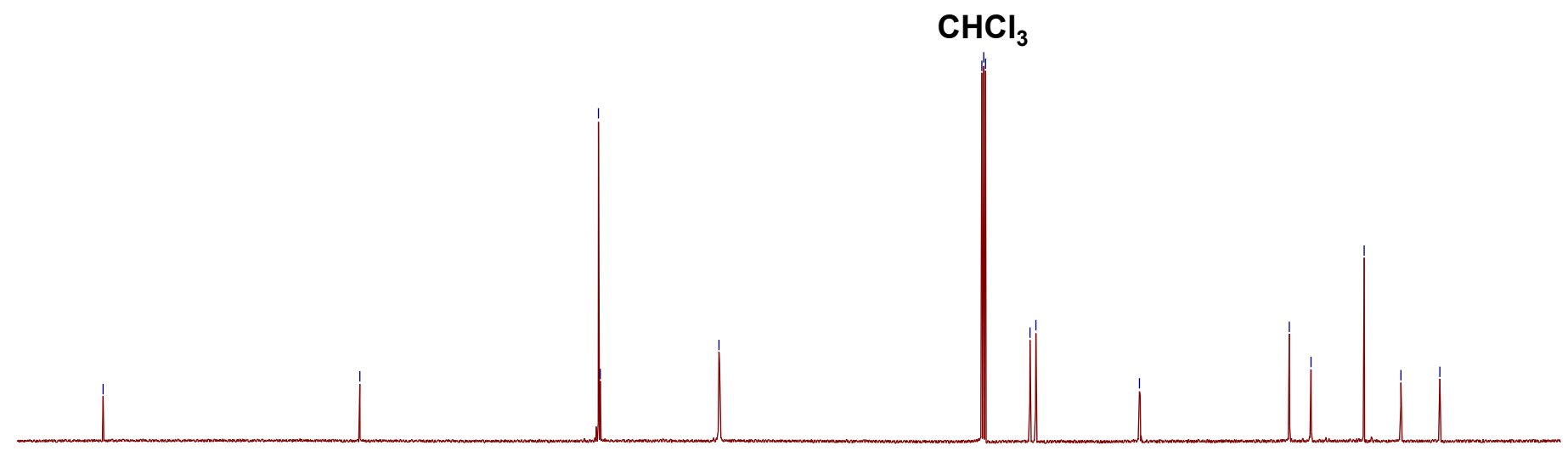

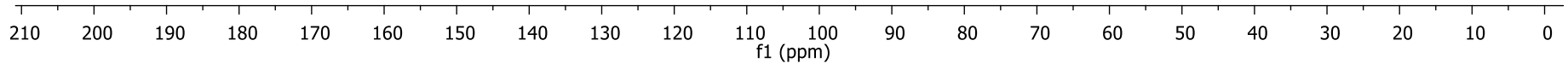


${ }^{1} \mathrm{H}-\mathrm{NMR}\left(300 \mathrm{MHz} ; \mathrm{CDCl}_{3}\right.$ )<smiles>COc1ccc(C(=O)C(Cl)C[C@H](I)OC[Hg])cc1</smiles>

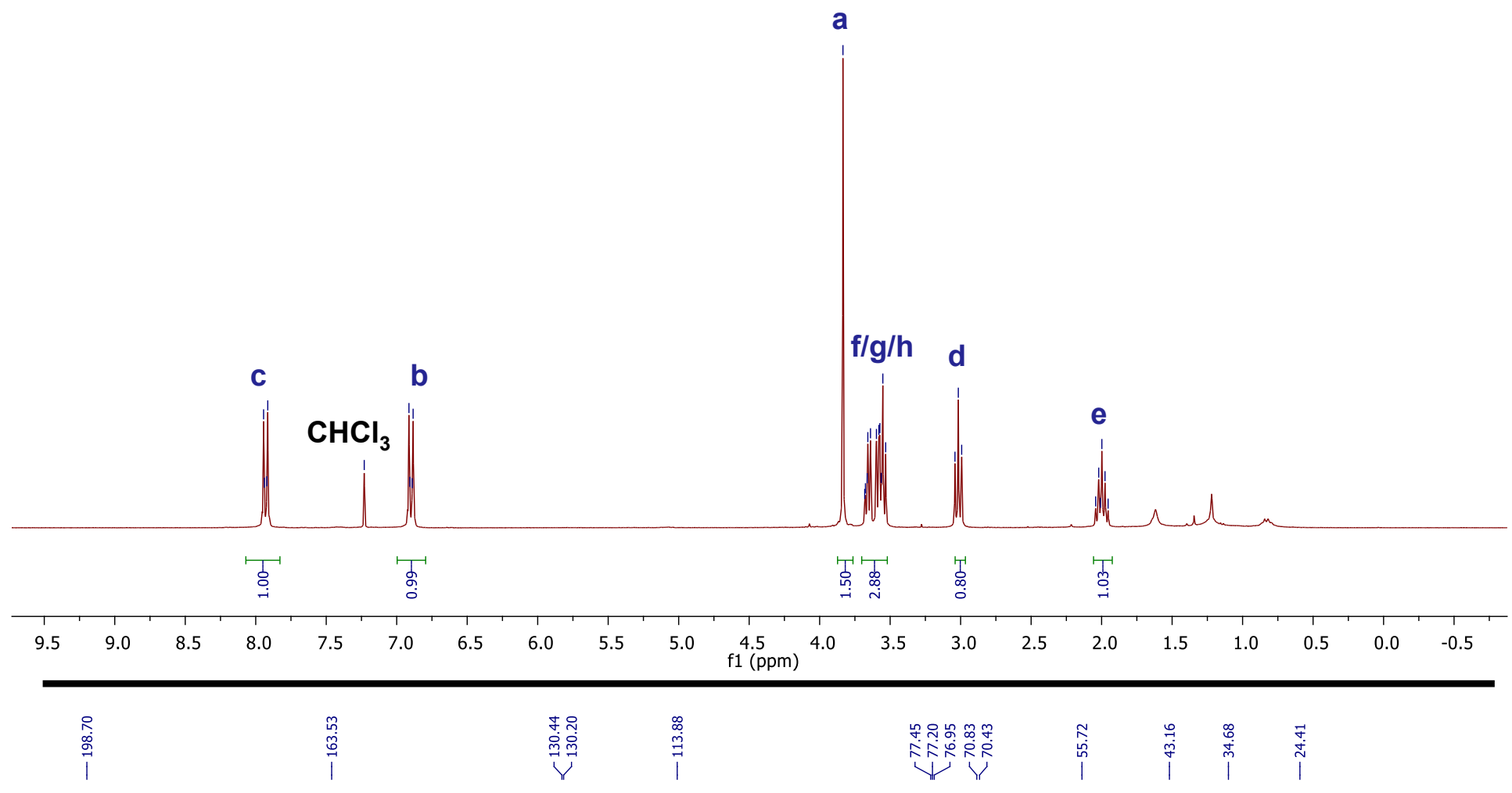

${ }^{13} \mathrm{C}-\mathrm{NMR}$ (126 MHz; $\mathrm{CDCl}_{3}$ )

\section{$\mathrm{CHCl}_{3}$}

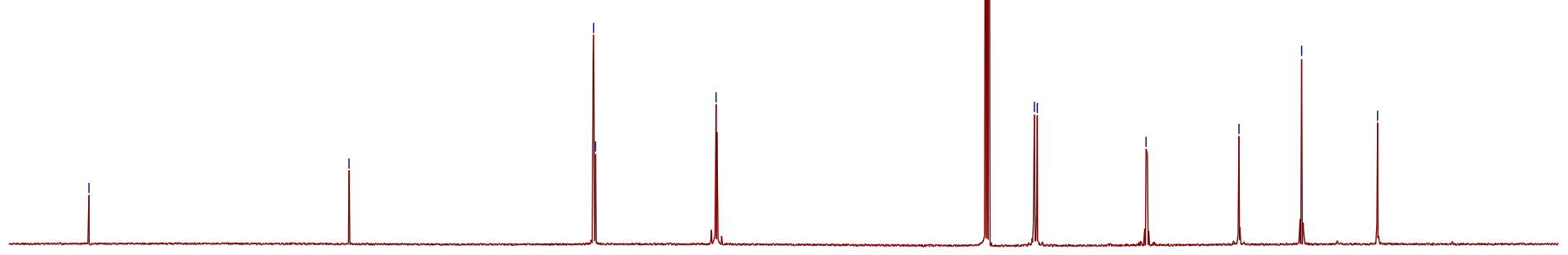


${ }^{1} \mathrm{H}-\mathrm{NMR}$ (300 MHz; $\mathrm{CDCl}_{3}$ )

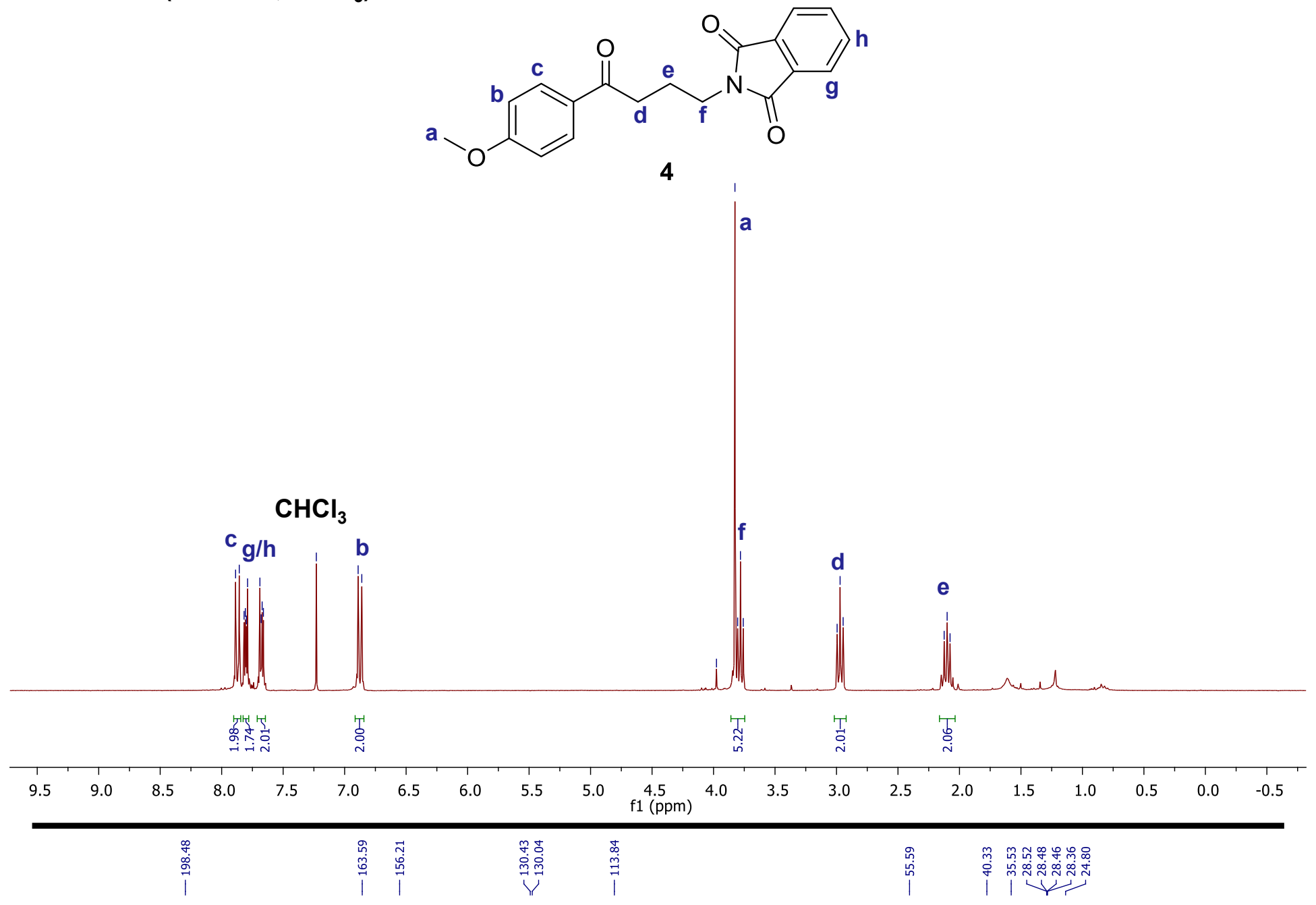

${ }^{13} \mathrm{C}-\mathrm{NMR}$ (126 MHz; $\mathrm{CDCl}_{3}$ )
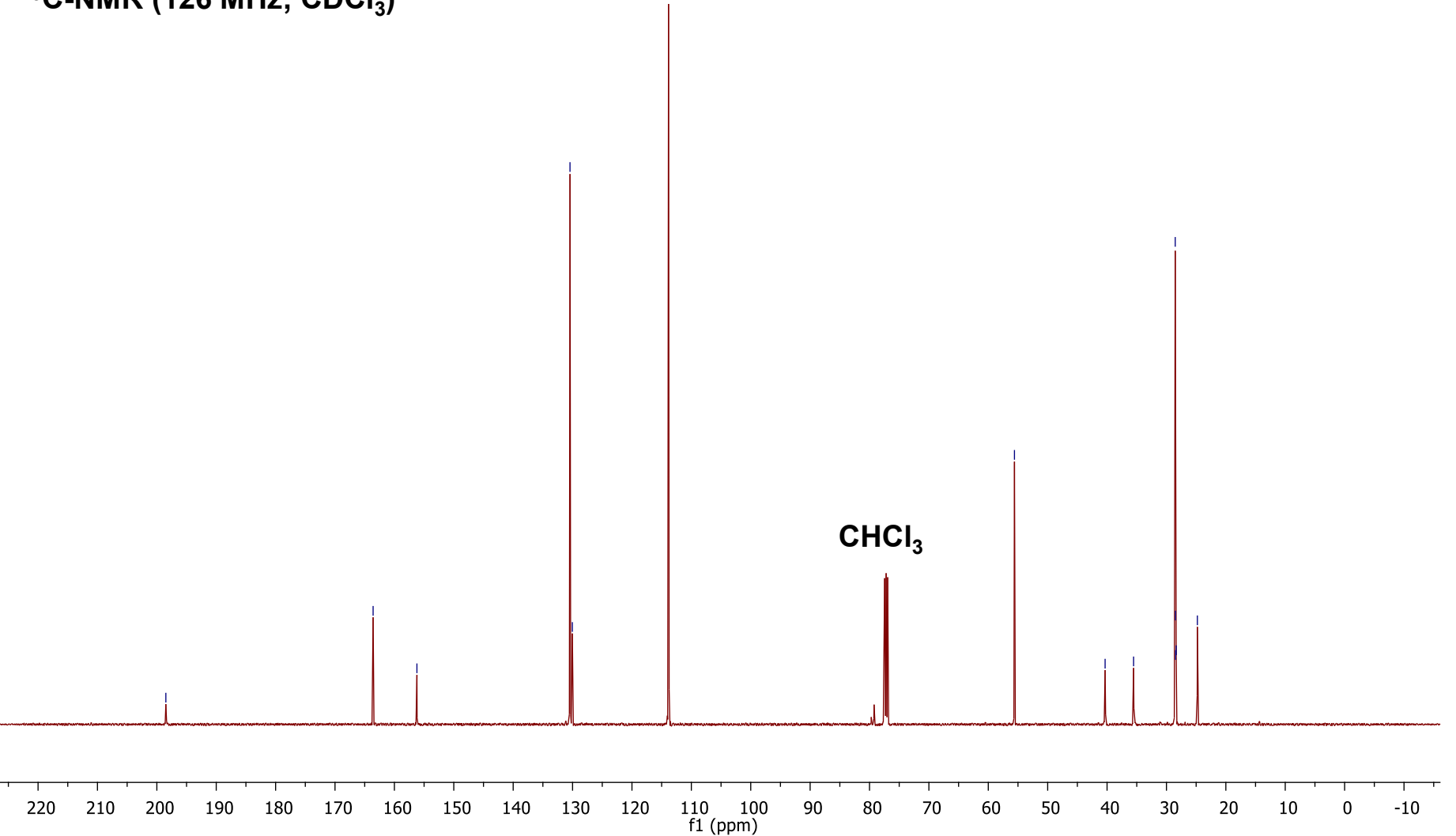

\section{S53}


1H-NMR (300 MHz; $\mathrm{CDCl}_{3}$ )

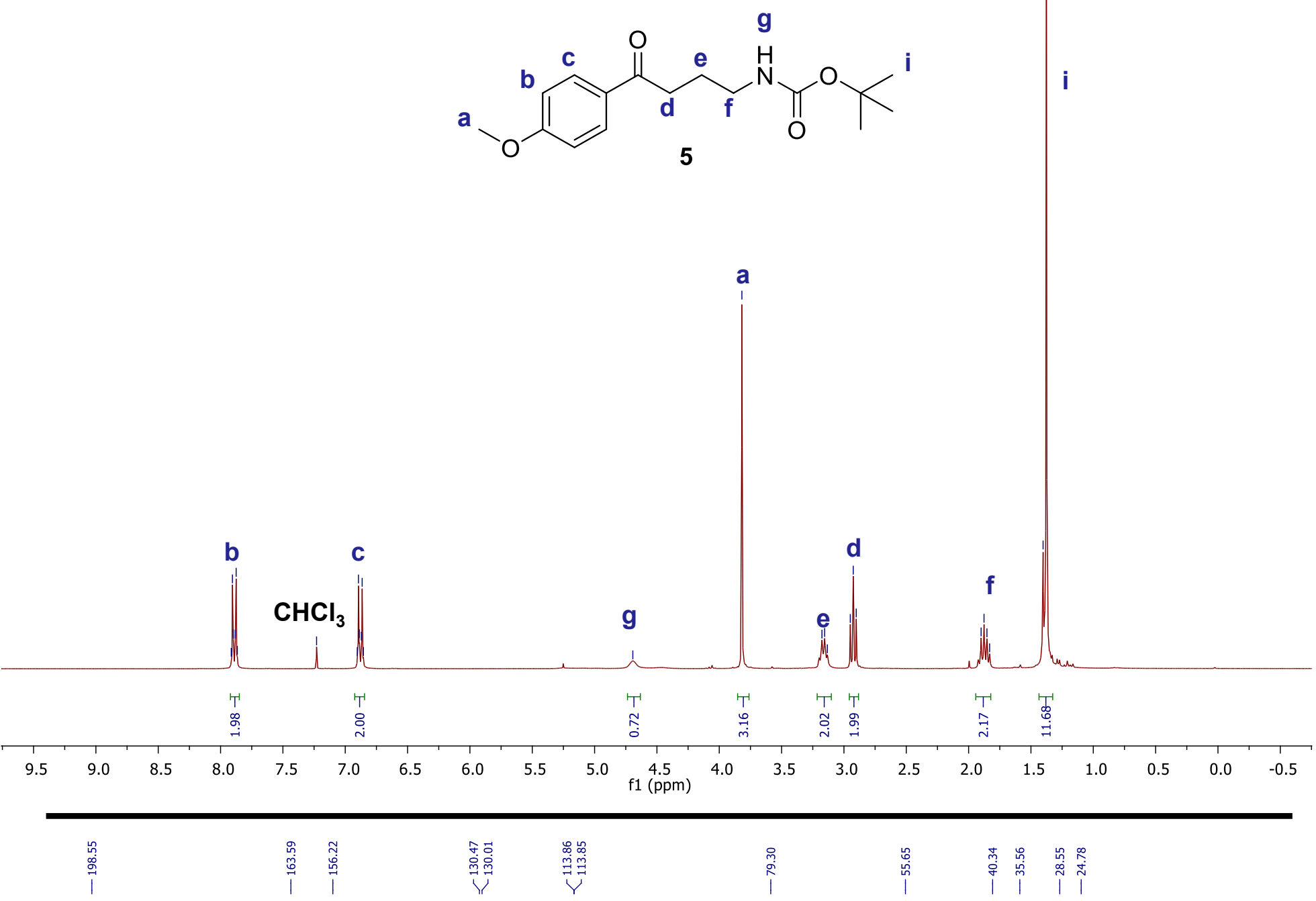

${ }^{13} \mathrm{C}-\mathrm{NMR}$ (126 MHz; $\mathrm{CDCl}_{3}$ )
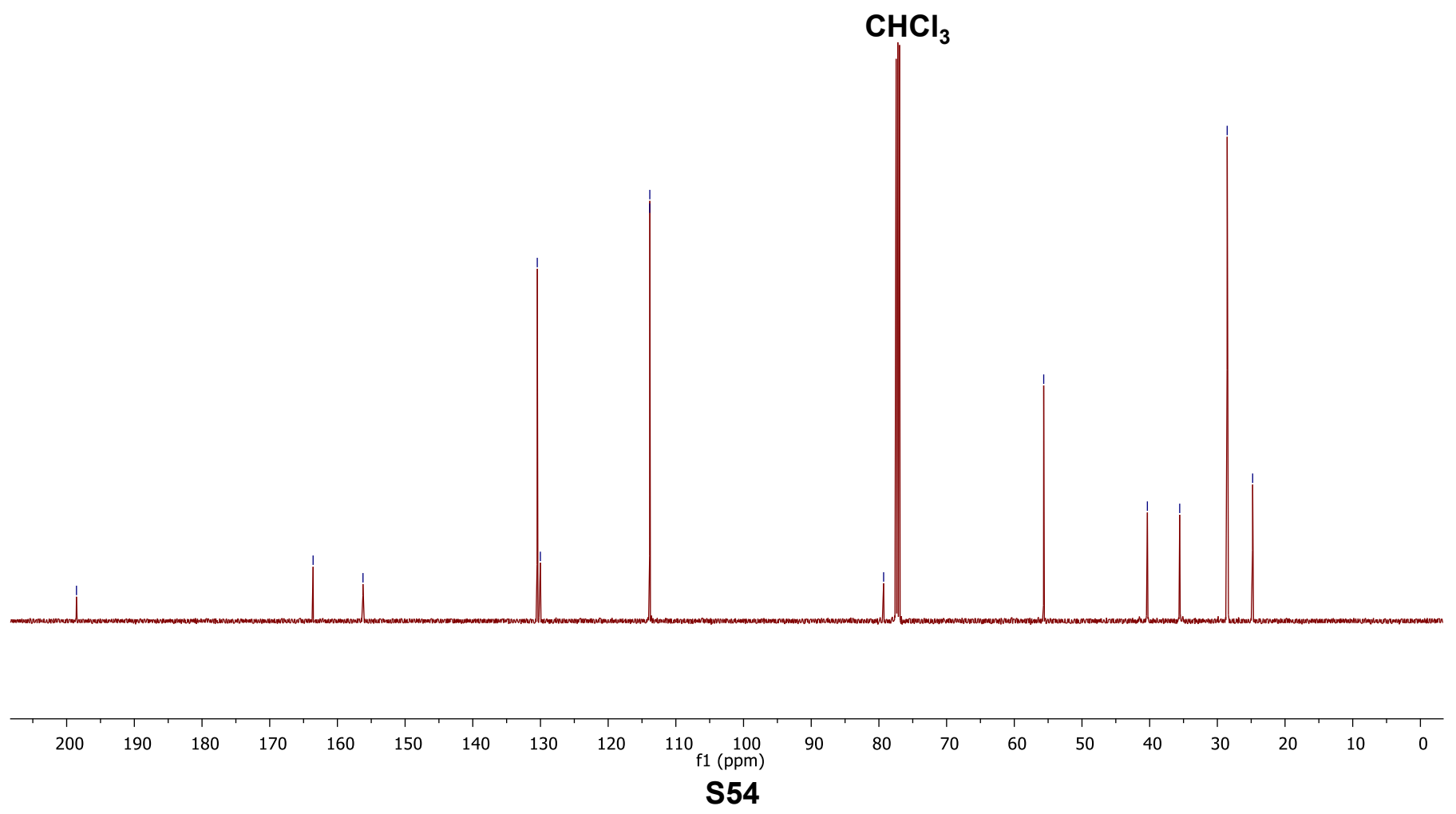
${ }^{1} \mathrm{H}-\mathrm{NMR}\left(300 \mathrm{MHz} ; \mathrm{CDCl}_{3}\right.$ )<smiles>COc1ccc(C(=O)CC[C](C)[Si](C)(C)[Hg])cc1</smiles>
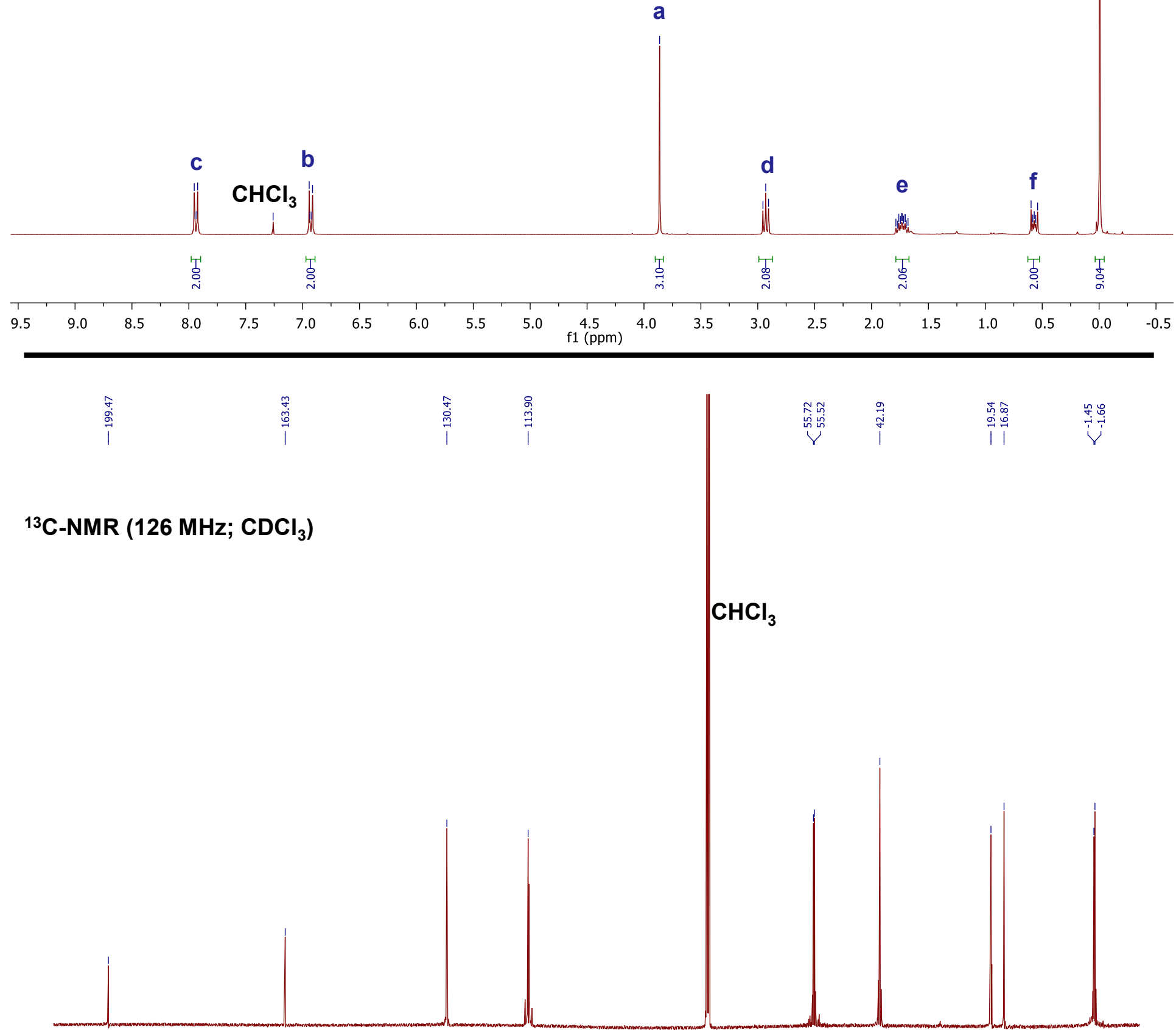
${ }^{1} \mathrm{H}-\mathrm{NMR}\left(300 \mathrm{MHz} ; \mathrm{CDCl}_{3}\right.$ )<smiles>[3H]Oc1ccc(C(=O)CCC(C)SCC)cc1</smiles>

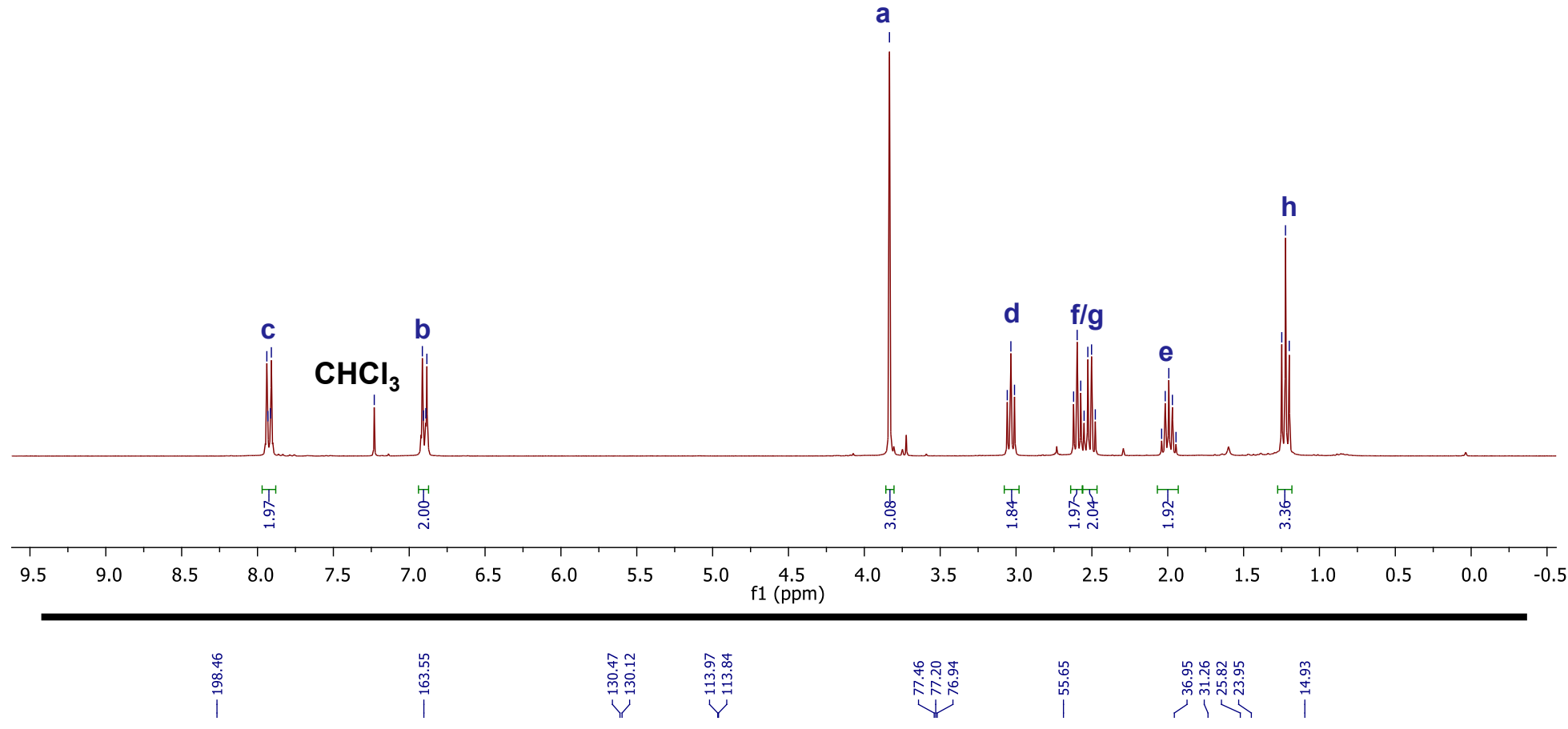

${ }^{13} \mathrm{C}-\mathrm{NMR}\left(126 \mathrm{MHz} ; \mathrm{CDCl}_{3}\right.$ )

$\mathrm{CHCl}_{3}$

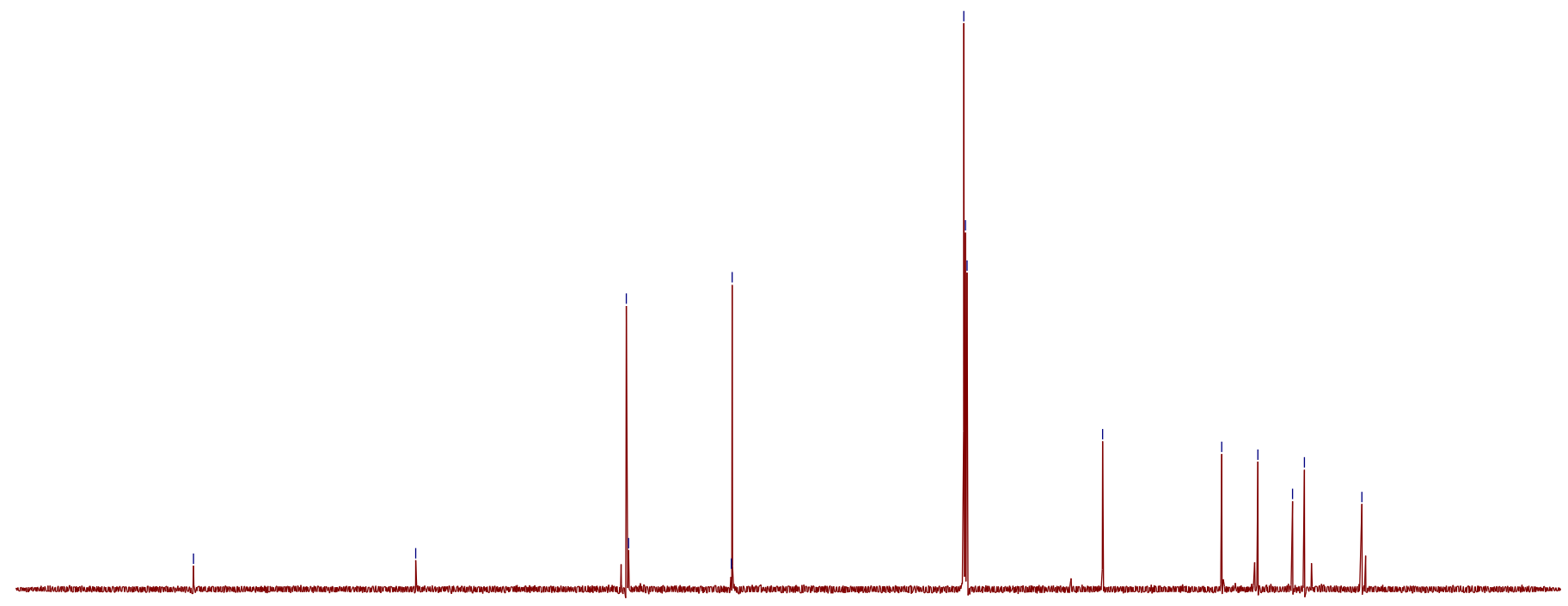


${ }^{1} \mathrm{H}-\mathrm{NMR}$ (300 MHz; $\mathrm{CDCl}_{3}$ )

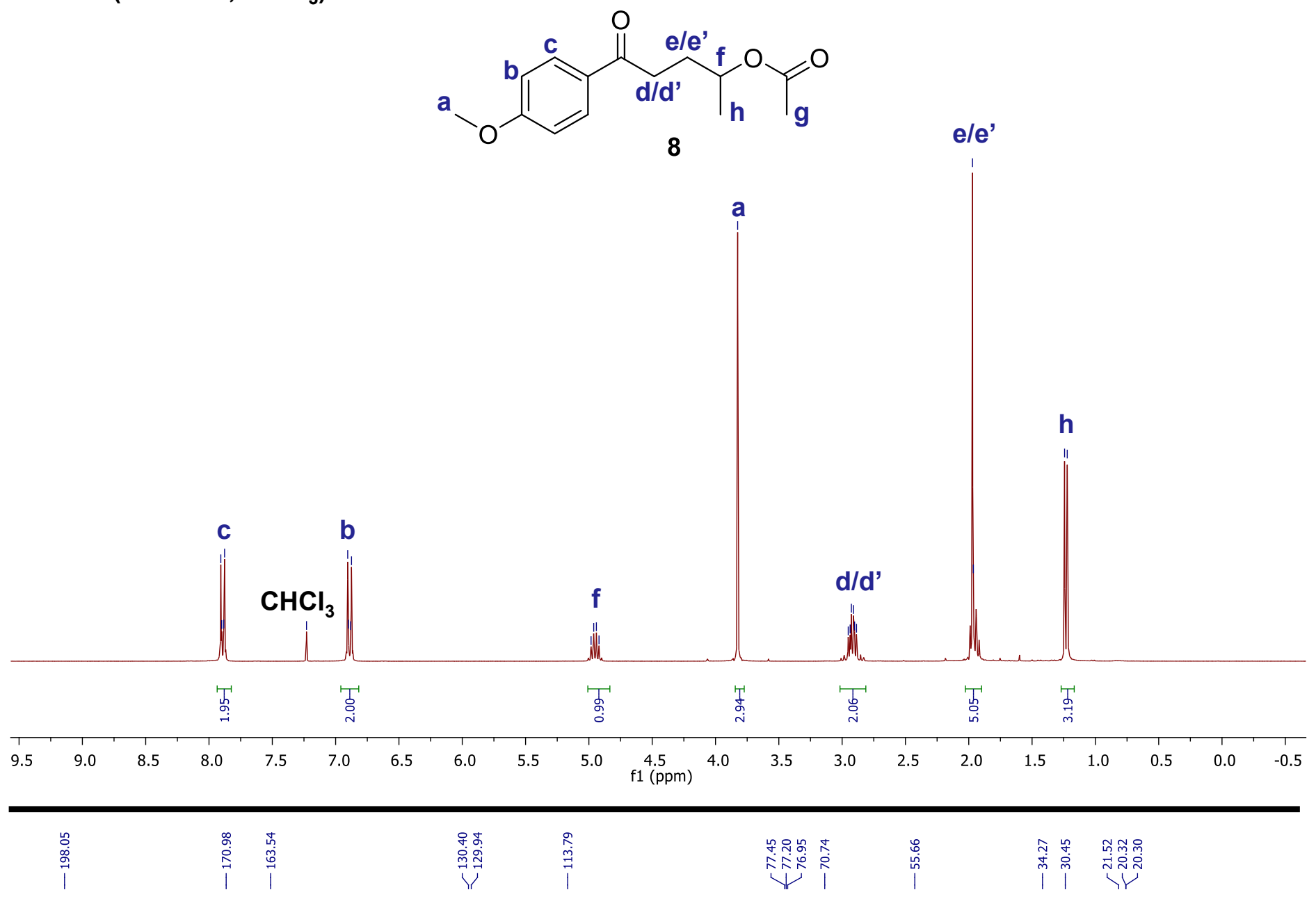

${ }^{13} \mathrm{C}-\mathrm{NMR}\left(126 \mathrm{MHz} ; \mathrm{CDCl}_{3}\right.$ )

\section{$\mathrm{CHCl}_{3}$}

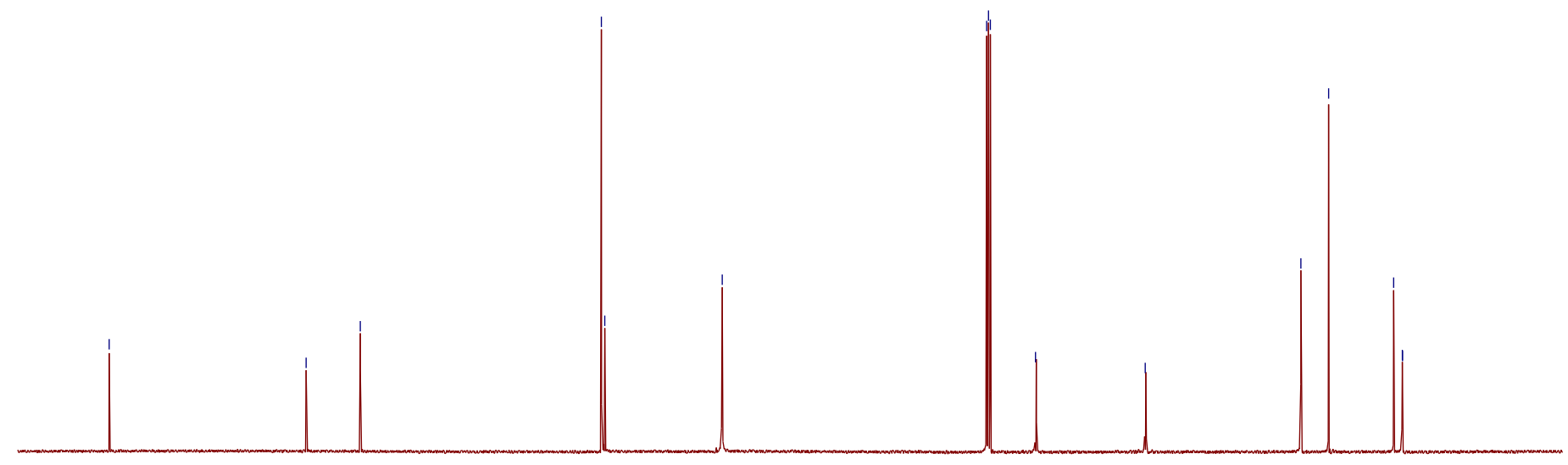

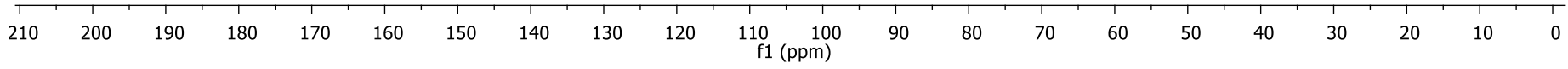


${ }^{1} \mathrm{H}-\mathrm{NMR}\left(300 \mathrm{MHz} ; \mathrm{CDCl}_{3}\right)$

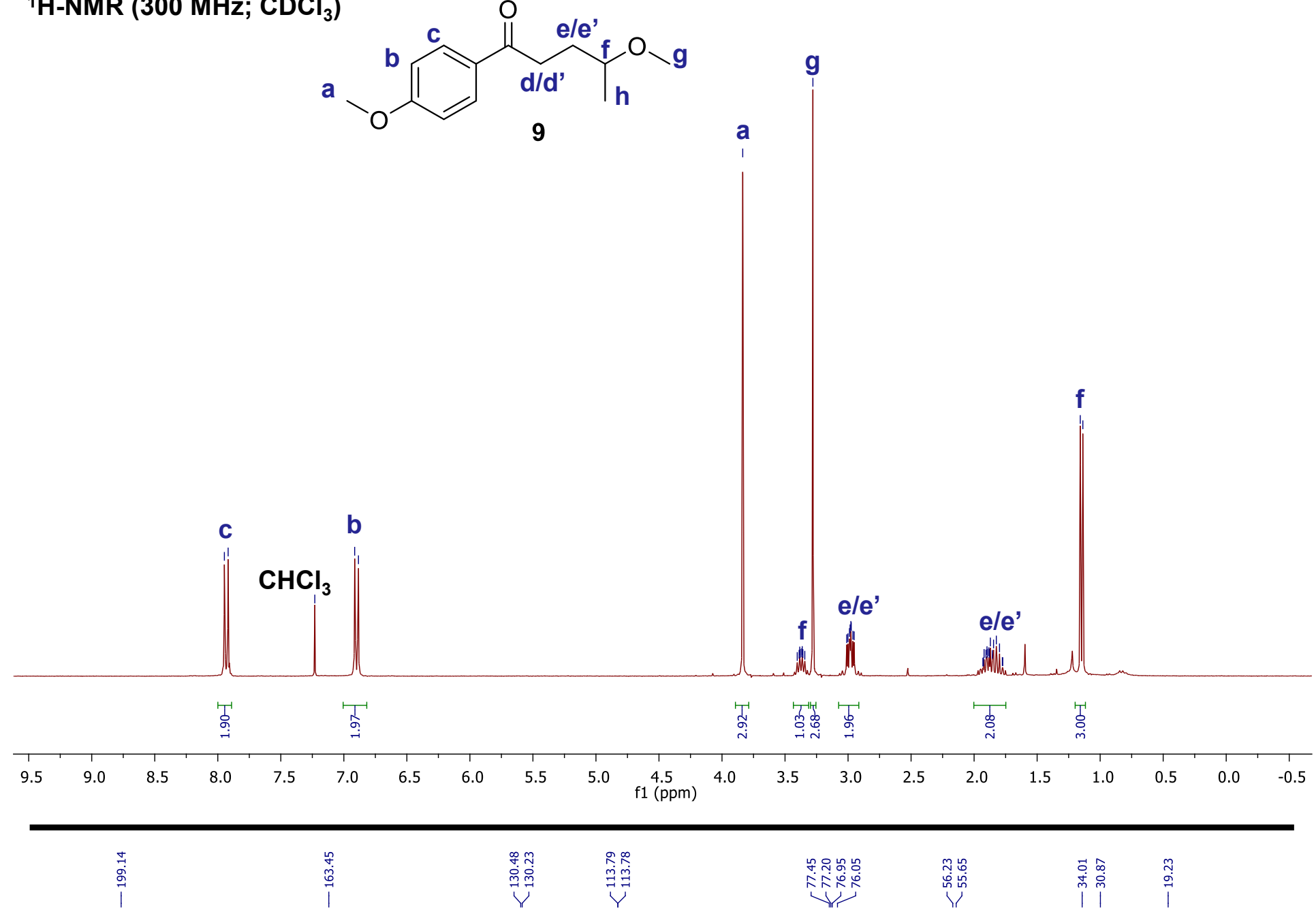

${ }^{13} \mathrm{C}-\mathrm{NMR}\left(126 \mathrm{MHz}^{\mathrm{CDCl}}{ }_{3}\right)$

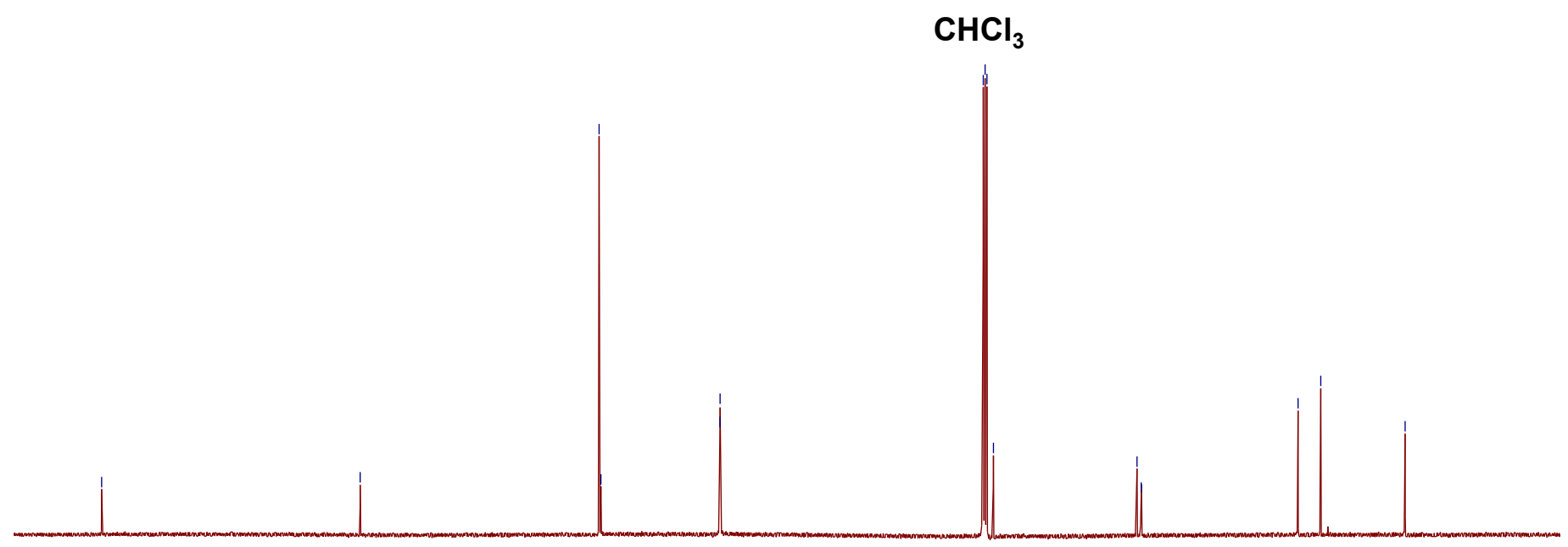

\begin{tabular}{|c|c|c|c|c|c|c|c|c|c|c|c|c|c|c|c|c|c|c|c|c|c|}
\hline 210 & 200 & 190 & 180 & 170 & 160 & 150 & 140 & 130 & 120 & $\begin{array}{l}110 \\
\mathrm{f} 1\end{array}$ & $\begin{array}{l}100 \\
\mathrm{pm})\end{array}$ & 90 & 80 & 70 & 60 & 50 & 40 & 30 & 20 & 10 & 0 \\
\hline
\end{tabular}


${ }^{1} \mathrm{H}-\mathrm{NMR}$ (300 MHz; $\mathrm{CDCl}_{3}$ )

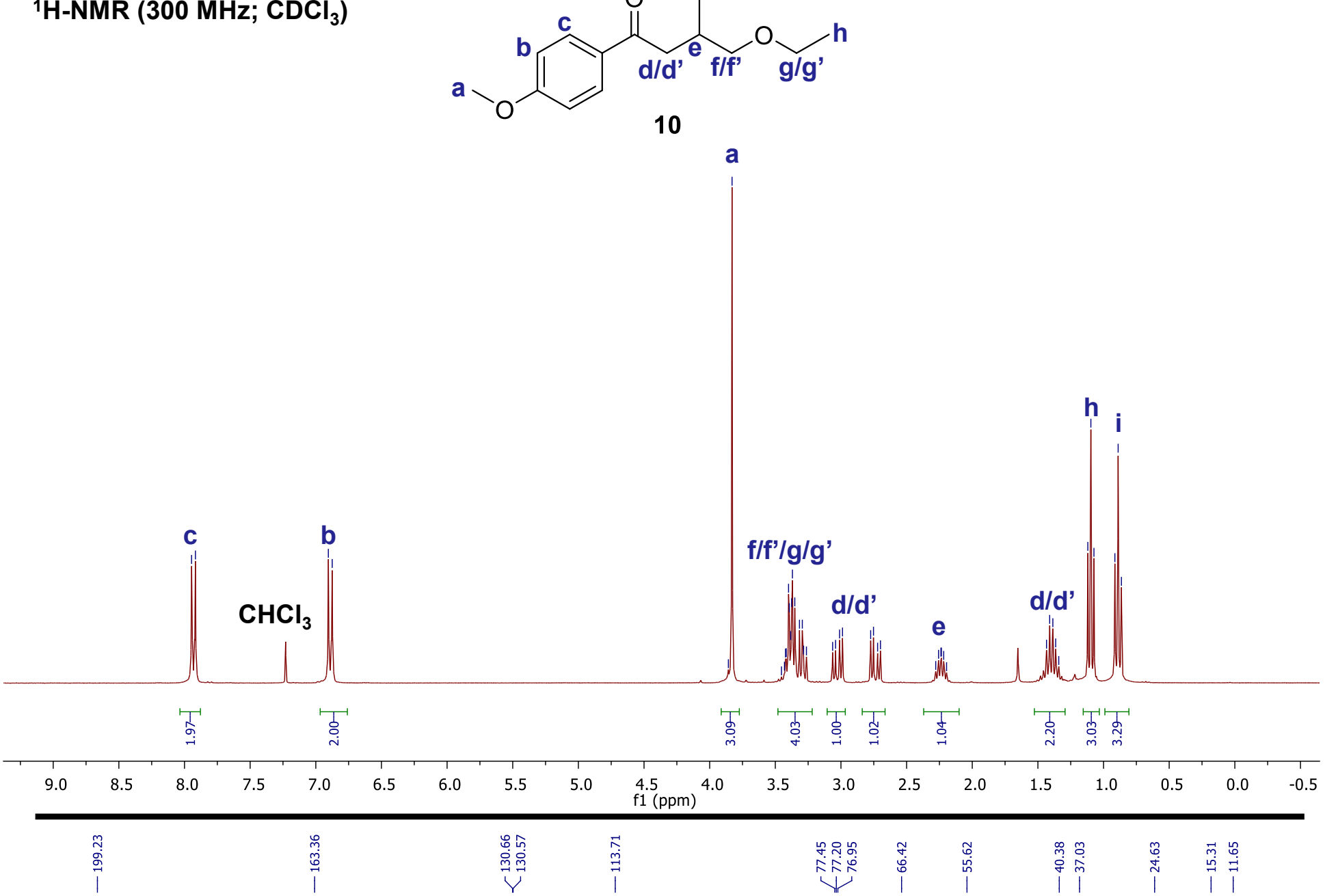

${ }^{13} \mathrm{C}-\mathrm{NMR}$ (126 MHz; $\mathrm{CDCl}_{3}$ )

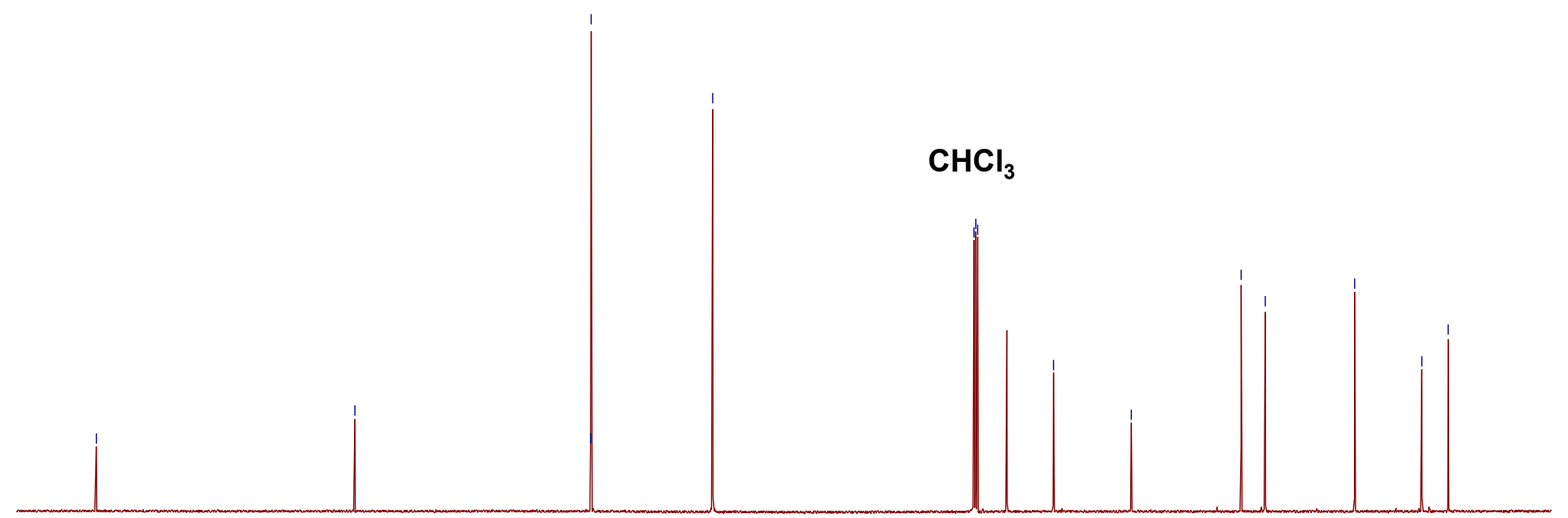

\begin{tabular}{|c|c|c|c|c|c|c|c|c|c|c|c|c|c|c|c|c|c|c|c|c|}
\hline$\Gamma$ & 1 & 1 & $T$ & $T$ & $T$ & $T$ & $T$ & $T$ & $T$ & $T$ & $T$ & $T$ & $T$ & $T$ & $T$ & $T$ & $T$ & $T$ & $T$ & $T$ \\
\hline 210 & 200 & 190 & 180 & 170 & 160 & 150 & 140 & 130 & 120 & $\begin{array}{c}110 \quad \begin{array}{c}100 \\
\mathrm{f} 1 \\
\mathbf{S 5 9}\end{array}\end{array}$ & 90 & 80 & 70 & 60 & 50 & 40 & 30 & 20 & 10 & 0 \\
\hline
\end{tabular}


${ }^{1} \mathrm{H}-\mathrm{NMR}\left(300 \mathrm{MHz} ; \mathrm{CDCl}_{3}\right)$

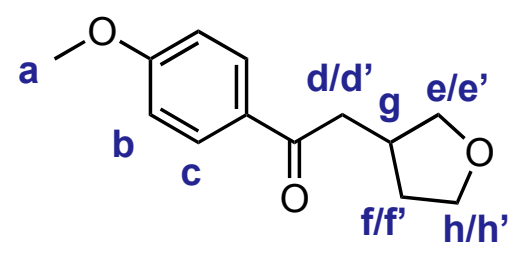

11

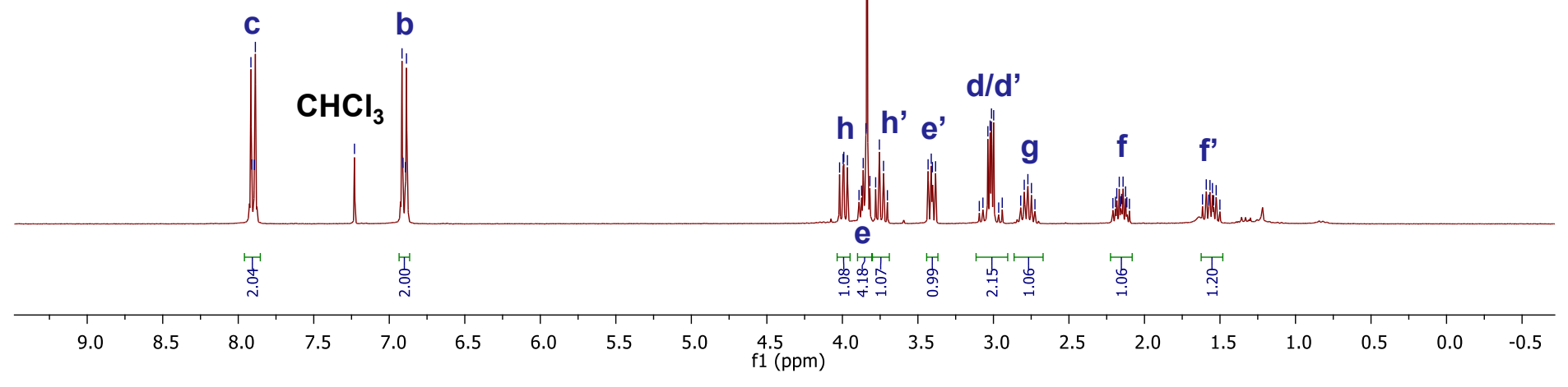

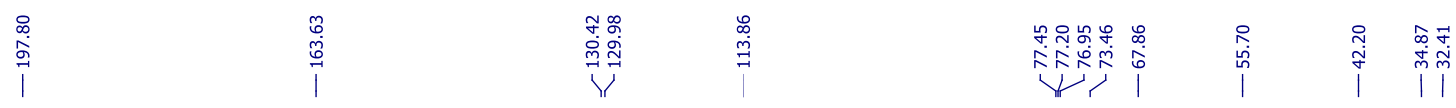

${ }^{13} \mathrm{C}-\mathrm{NMR}\left(126 \mathrm{MHz}^{\mathrm{CDCl}}{ }_{3}\right)$

$\mathrm{CHCl}_{3}$

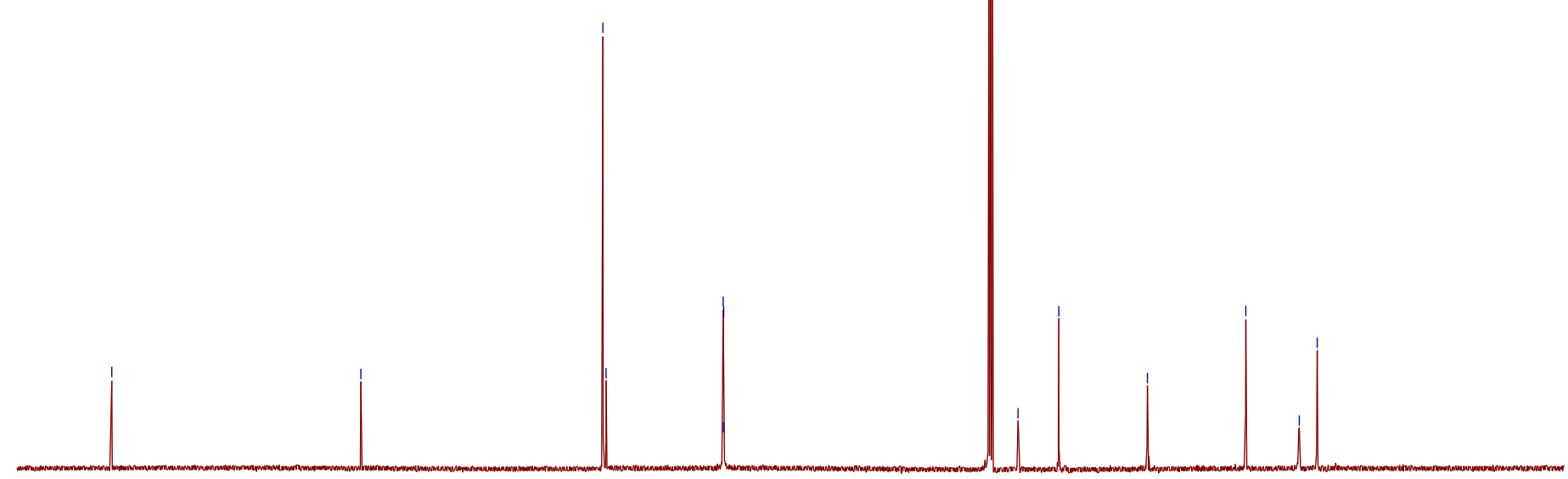

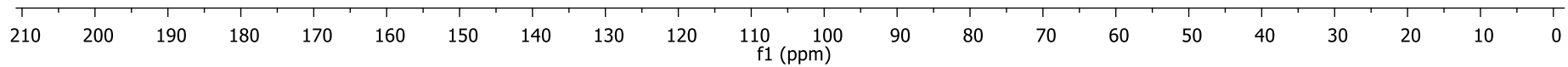


${ }^{1} \mathrm{H}-\mathrm{NMR}$ (300 MHz; $\mathrm{CDCl}_{3}$ )

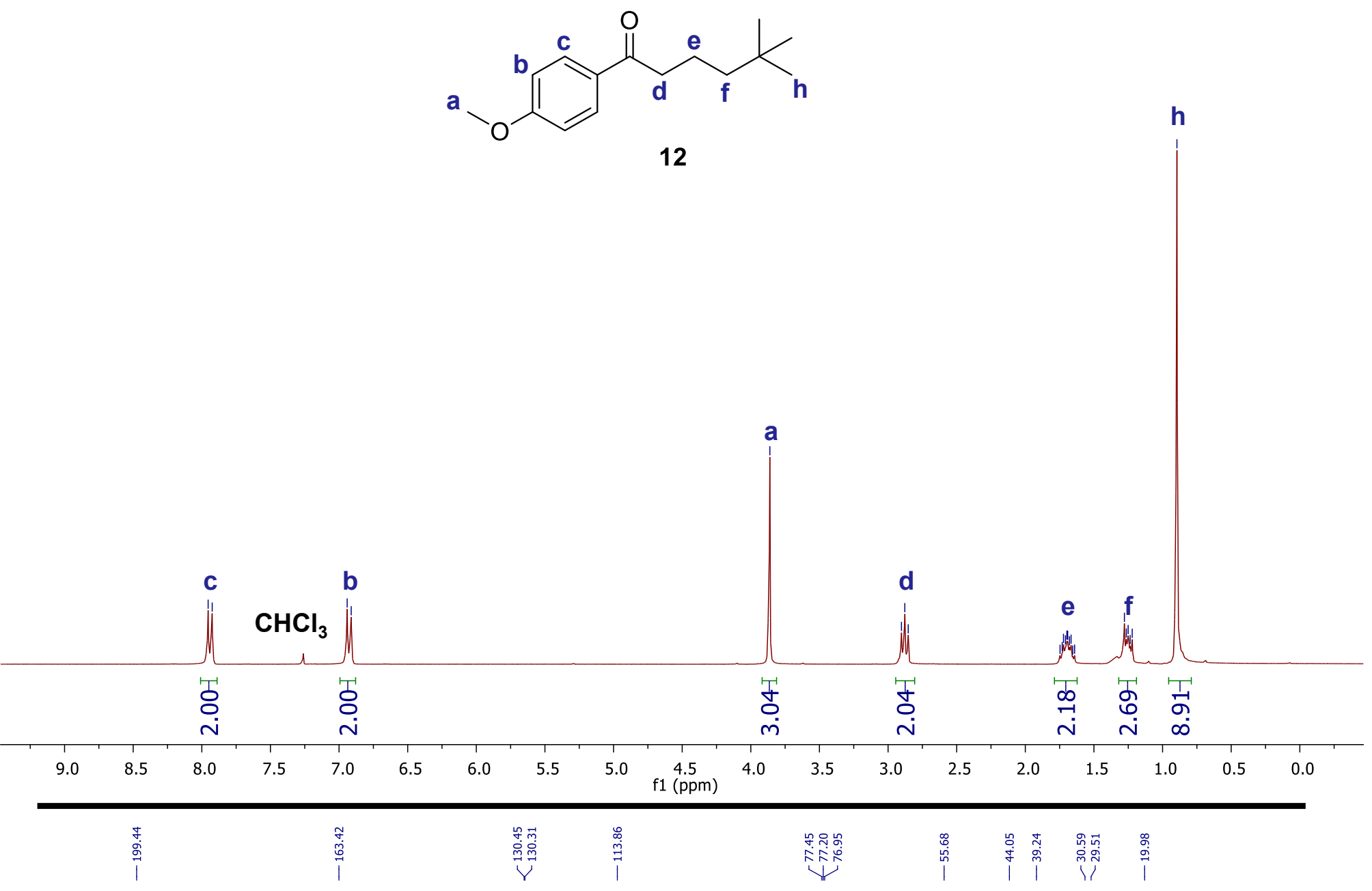

${ }^{13} \mathrm{C}-\mathrm{NMR}$ (126 MHz; $\mathrm{CDCl}_{3}$ )
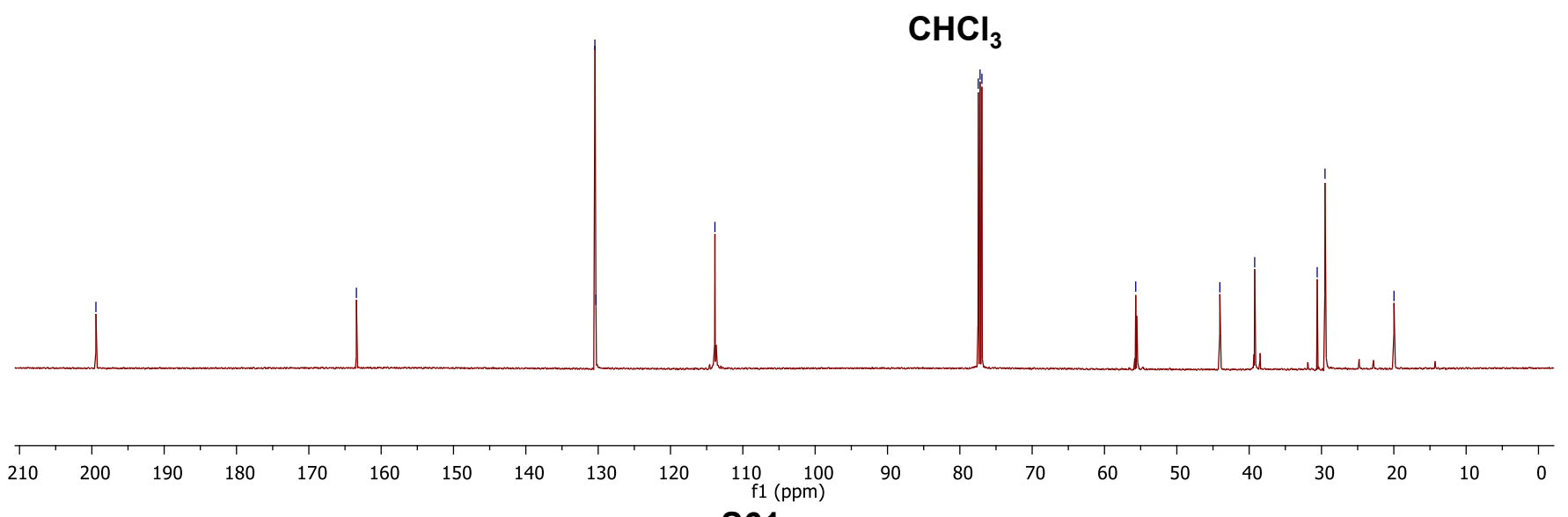
${ }^{1} \mathrm{H}-\mathrm{NMR}\left(300 \mathrm{MHz} ; \mathrm{CDCl}_{3}\right.$ )

a<smiles>COc1ccc(C(=O)CC(C)CC2=CC=IC=C2)cc1</smiles>

13

a

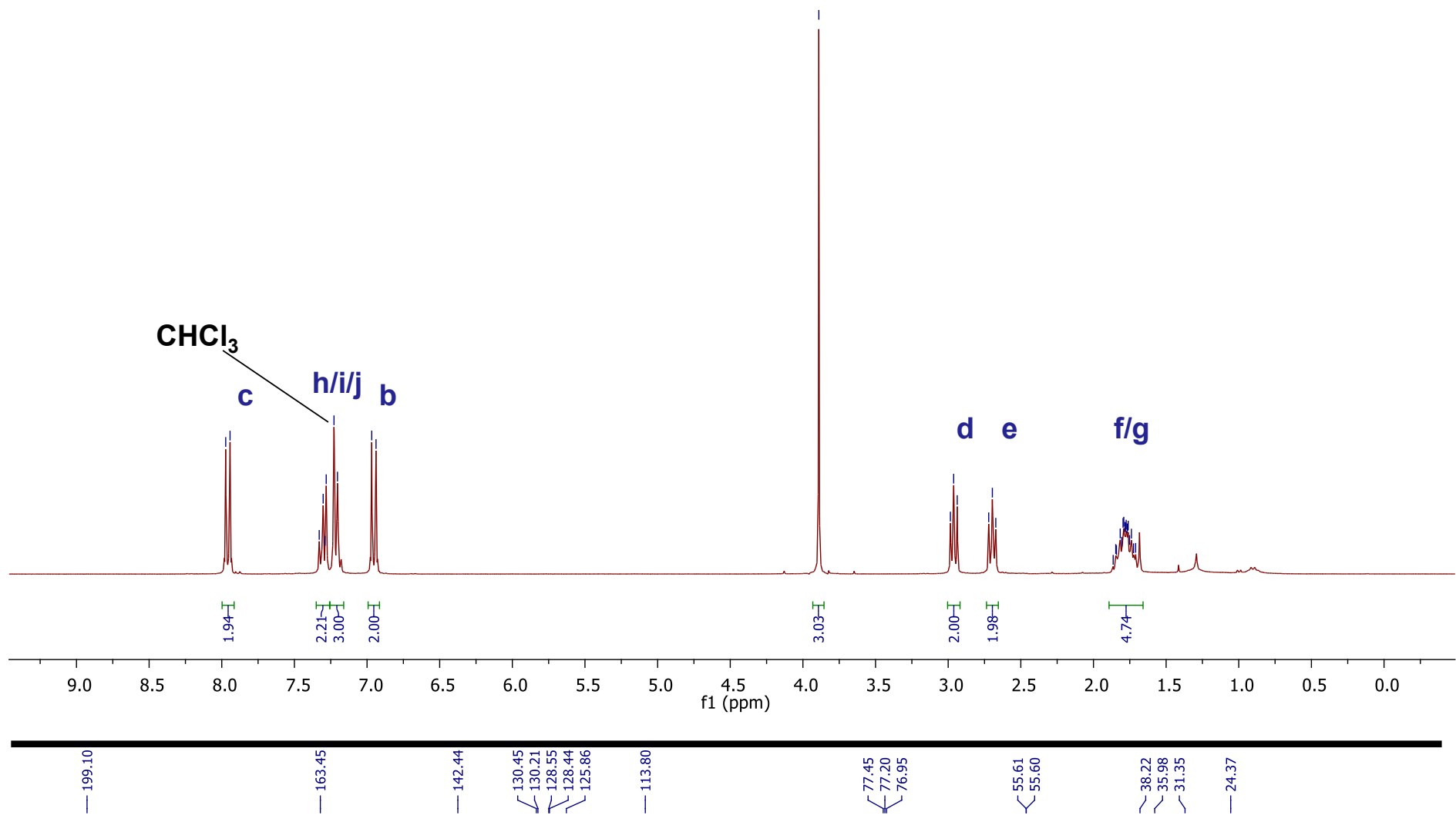

${ }^{13} \mathrm{C}-\mathrm{NMR}\left(126 \mathrm{MHz} ; \mathrm{CDCl}_{3}\right.$ )

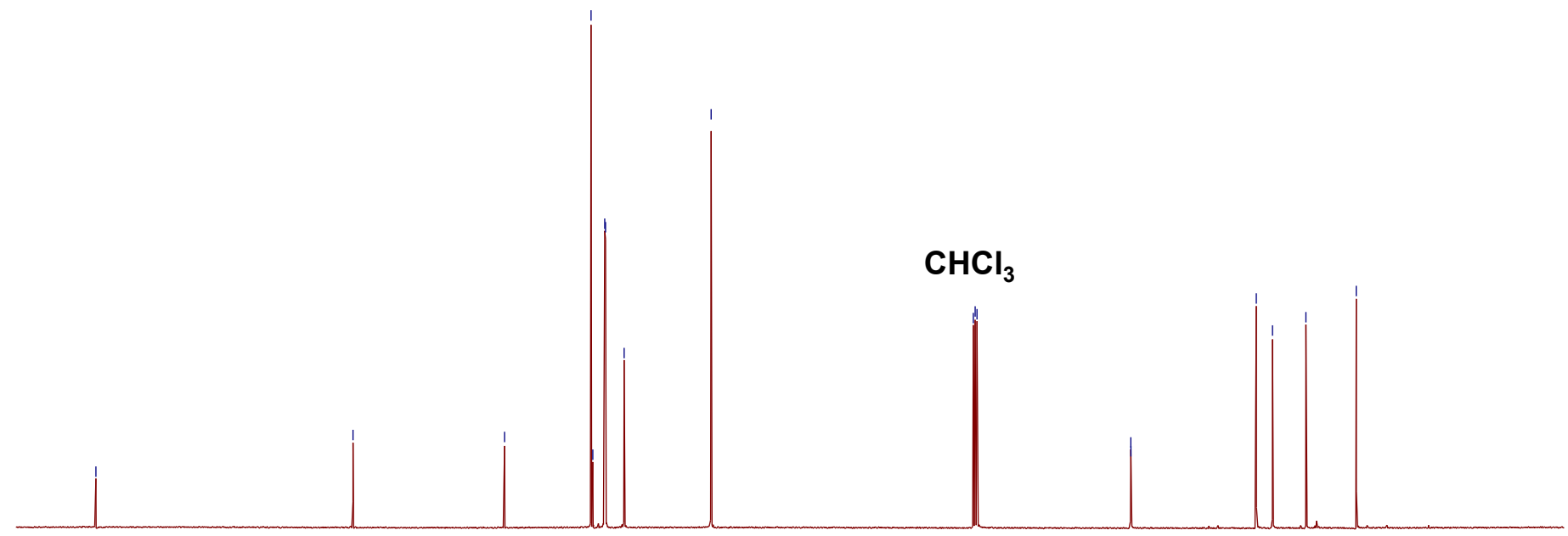


${ }^{1} \mathrm{H}-\mathrm{NMR}$ (300 MHz; $\mathrm{CDCl}_{3}$ )

a<smiles>COc1ccc(C(=O)CCC(C)CCC(C)C)cc1</smiles>

14

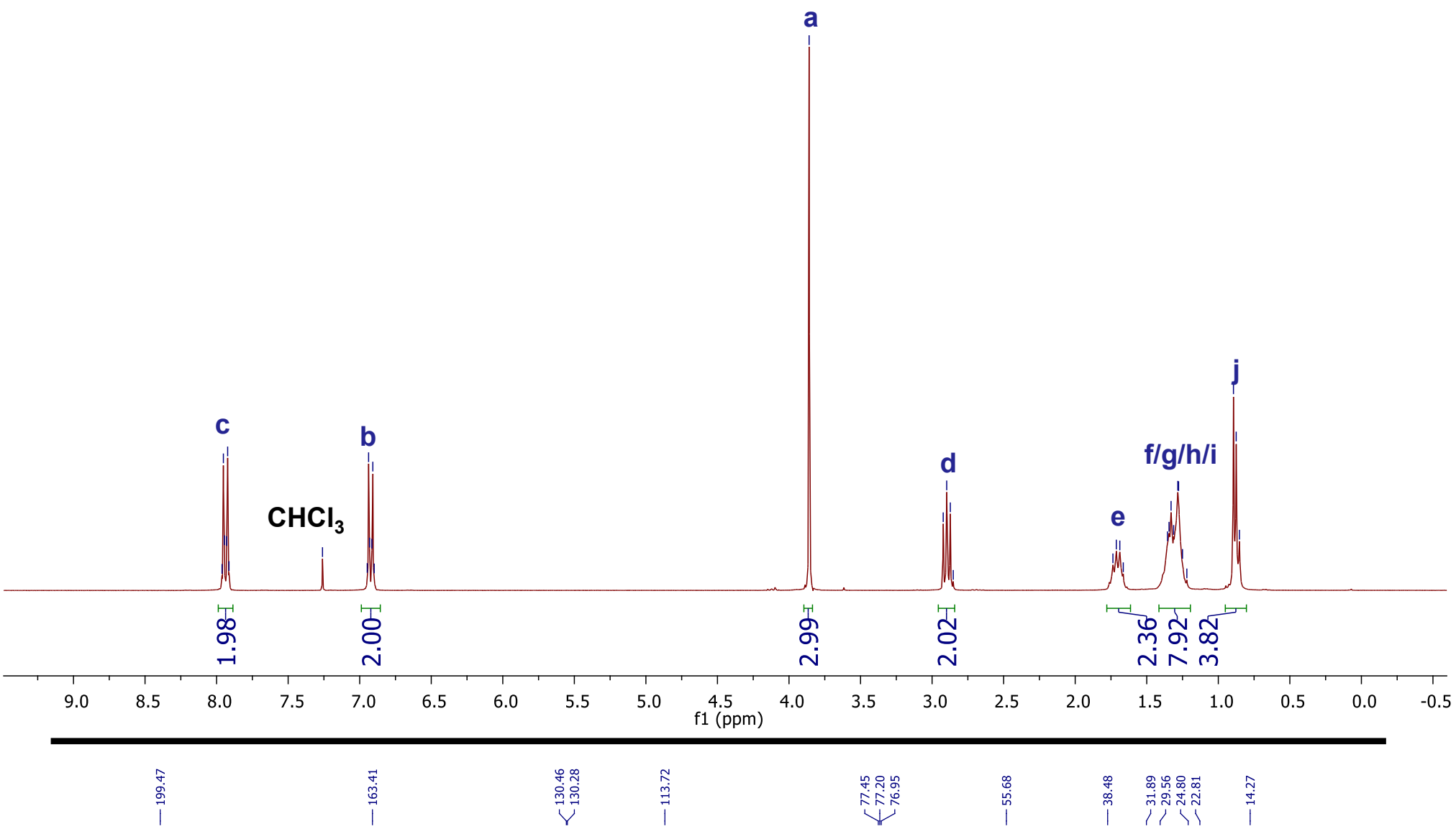

${ }^{13} \mathrm{C}-\mathrm{NMR}$ (126 MHz; $\mathrm{CDCl}_{3}$ )

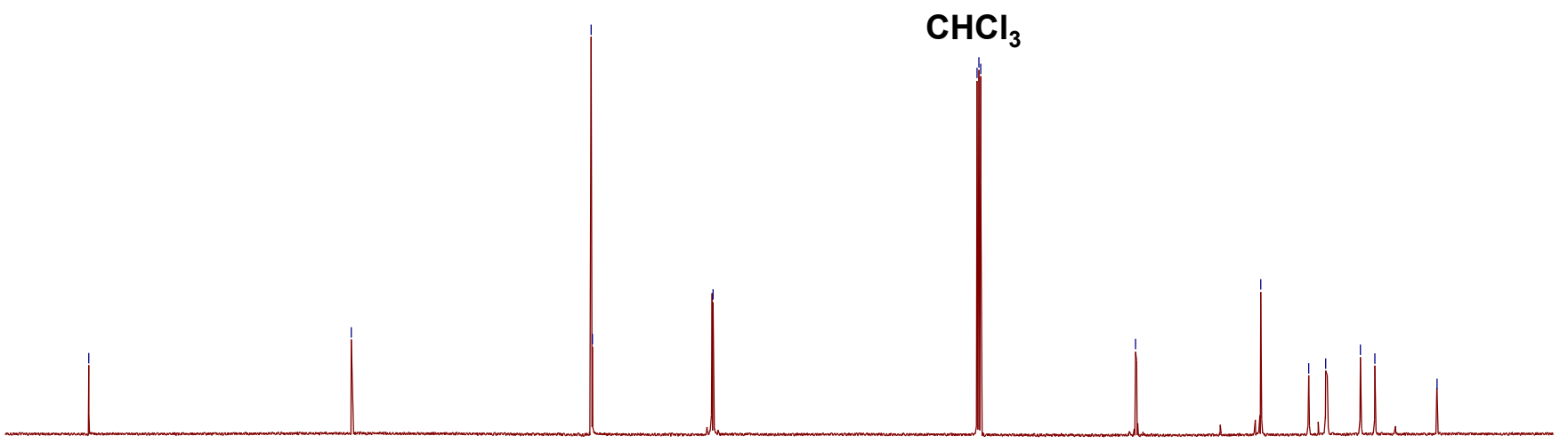

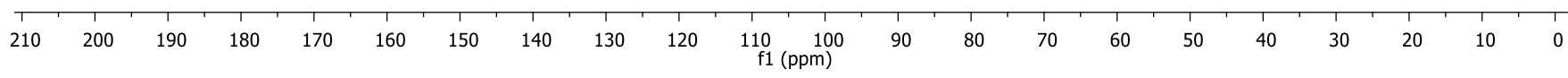


${ }^{1} \mathrm{H}-\mathrm{NMR}\left(300 \mathrm{MHz} ; \mathrm{CDCl}_{3}\right)$

a<smiles>COc1ccc(C(=O)C(Cl)CCC=CC(C)O)cc1</smiles>

15

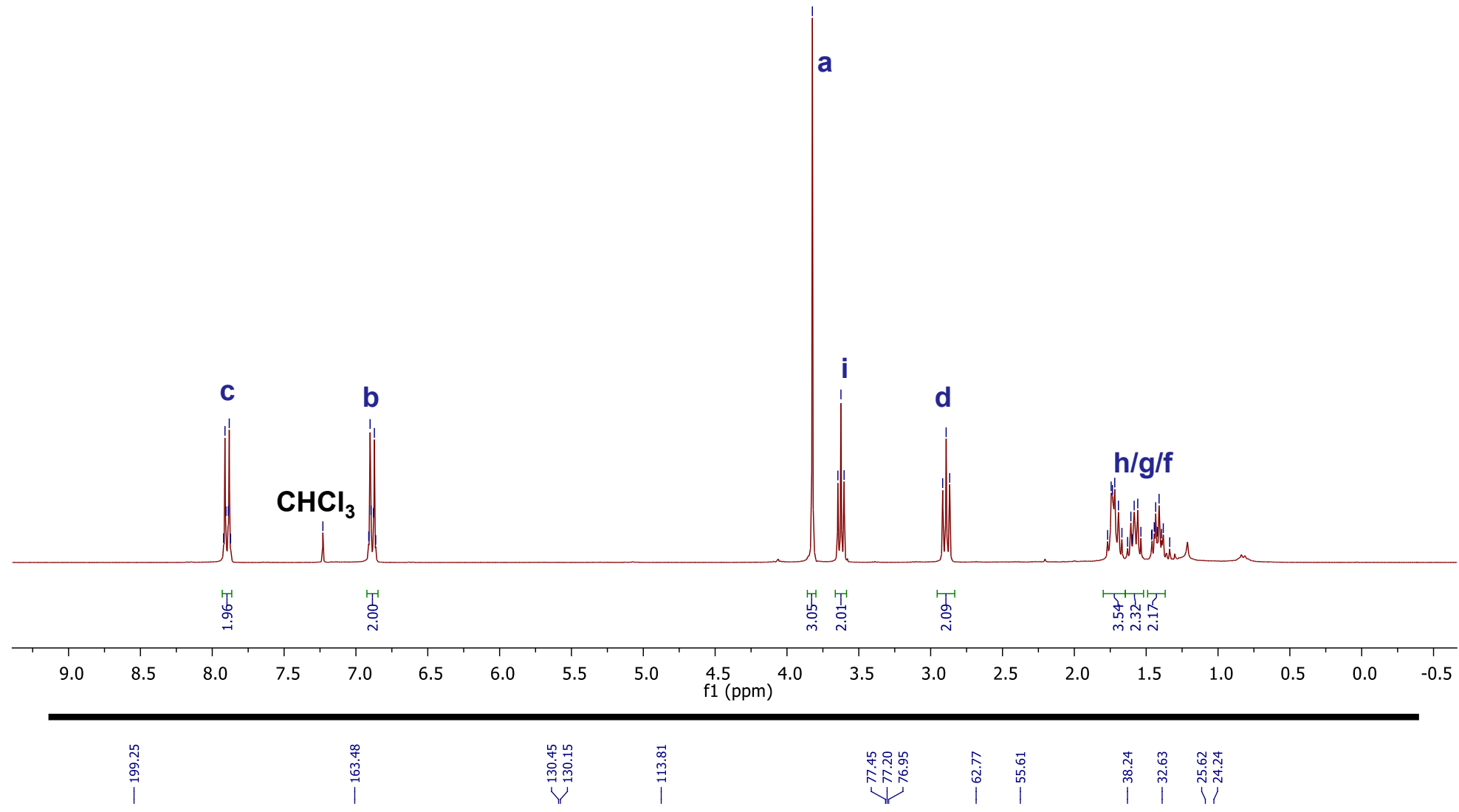

${ }^{13} \mathrm{C}-\mathrm{NMR}\left(126 \mathrm{MHz} ; \mathrm{CDCl}_{3}\right)$

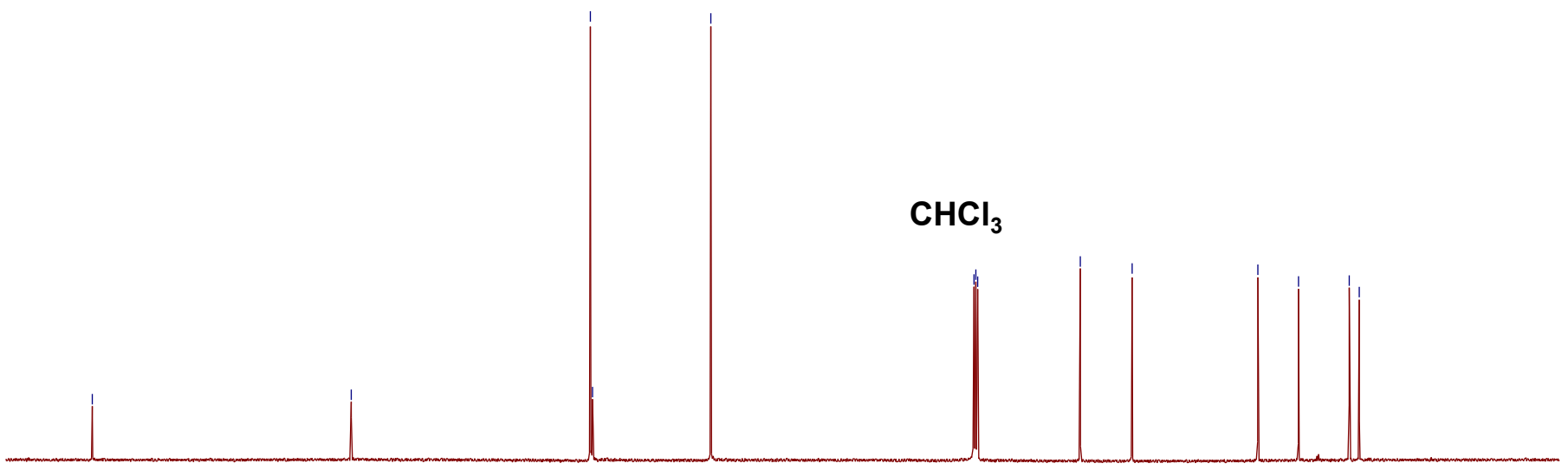

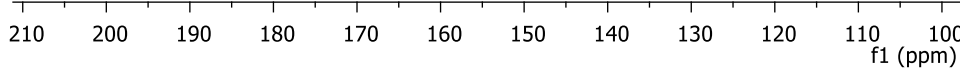


${ }^{1} \mathrm{H}-\mathrm{NMR}\left(300 \mathrm{MHz} ; \mathrm{CDCl}_{3}\right)$<smiles>COc1ccc(C(=O)CCCCCOC(=O)I)cc1</smiles>

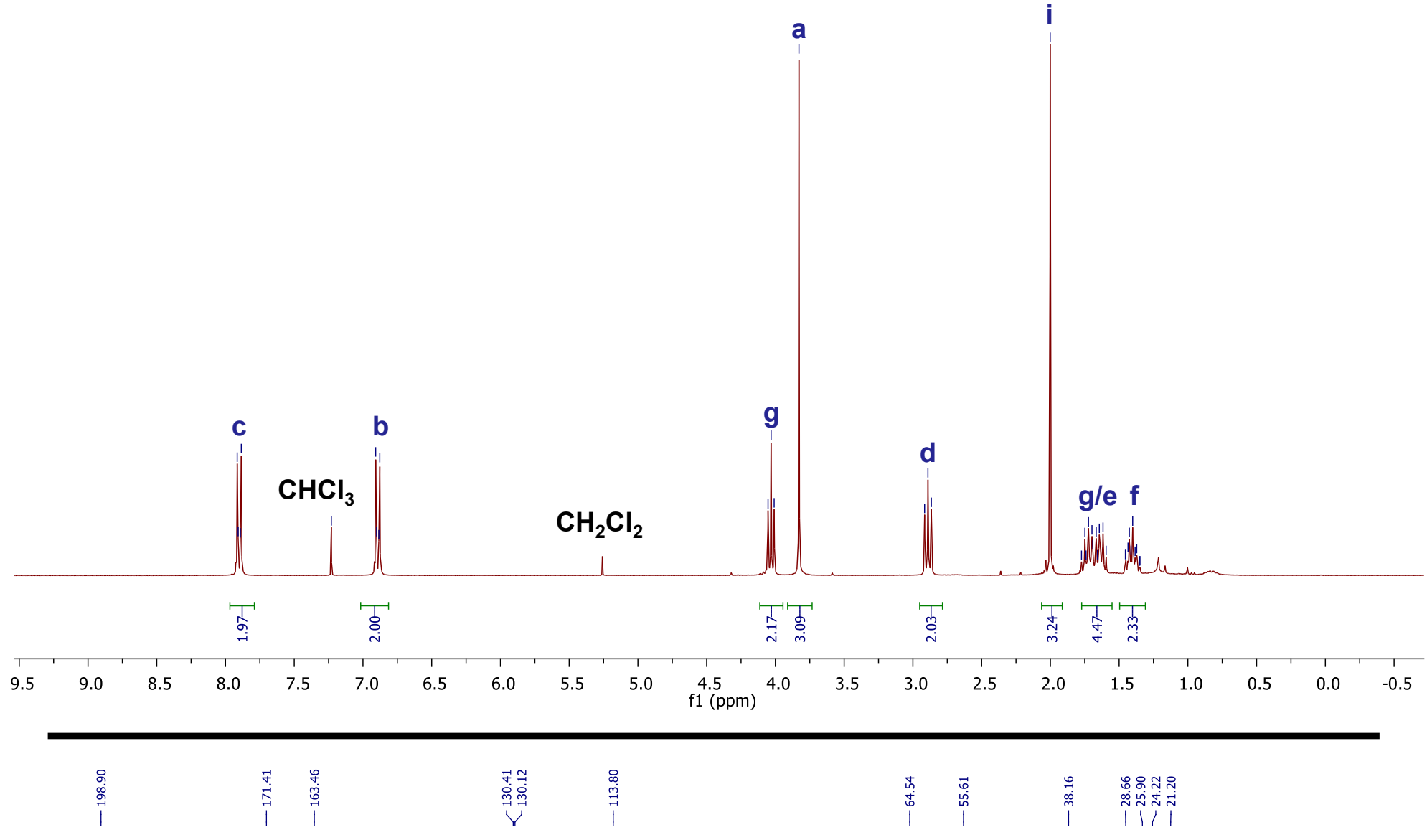

${ }^{13} \mathrm{C}-\mathrm{NMR}\left(126 \mathrm{MHz} ; \mathrm{CDCl}_{3}\right.$ )

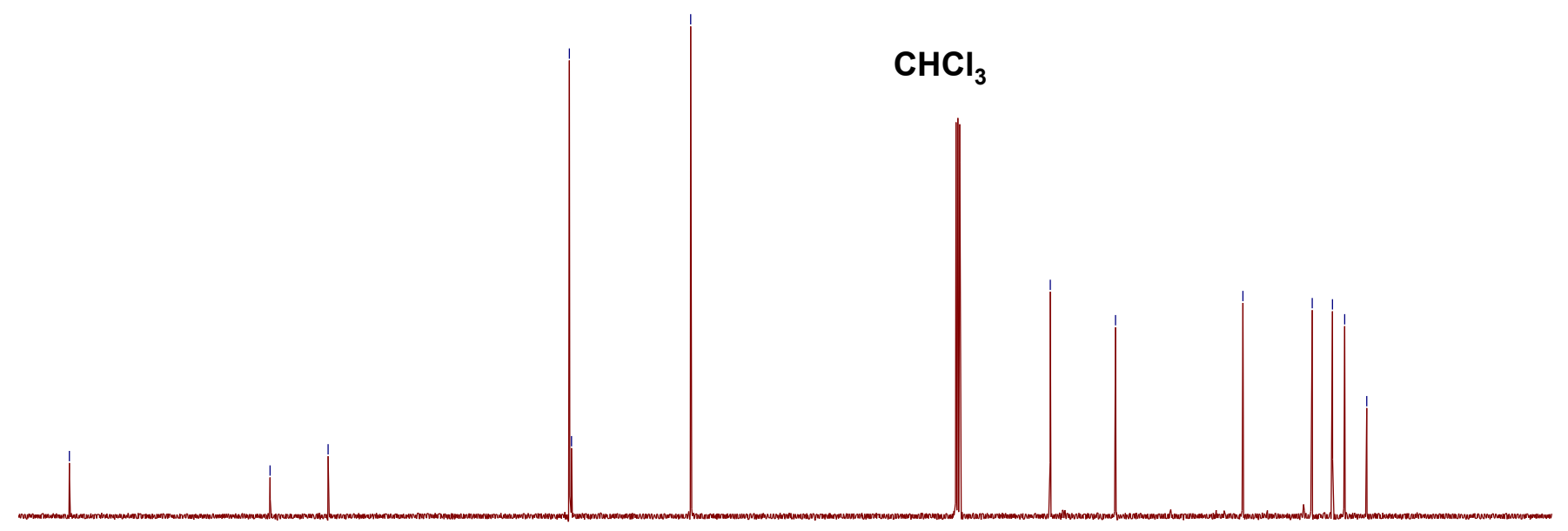

$\begin{array}{llllllllllllllllllllllll}200 & 190 & 180 & 170 & 160 & 150 & 140 & 130 & 120 & 110 & \begin{array}{c}100 \\ \mathrm{f} 1(\mathrm{ppm})\end{array} & 90 & 80 & 70 & 60 & 50 & 40 & 30 & 20 & 10 & 0\end{array}$


${ }^{1} \mathrm{H}-\mathrm{NMR}\left(300 \mathrm{MHz} ; \mathrm{CDCl}_{3}\right.$ )

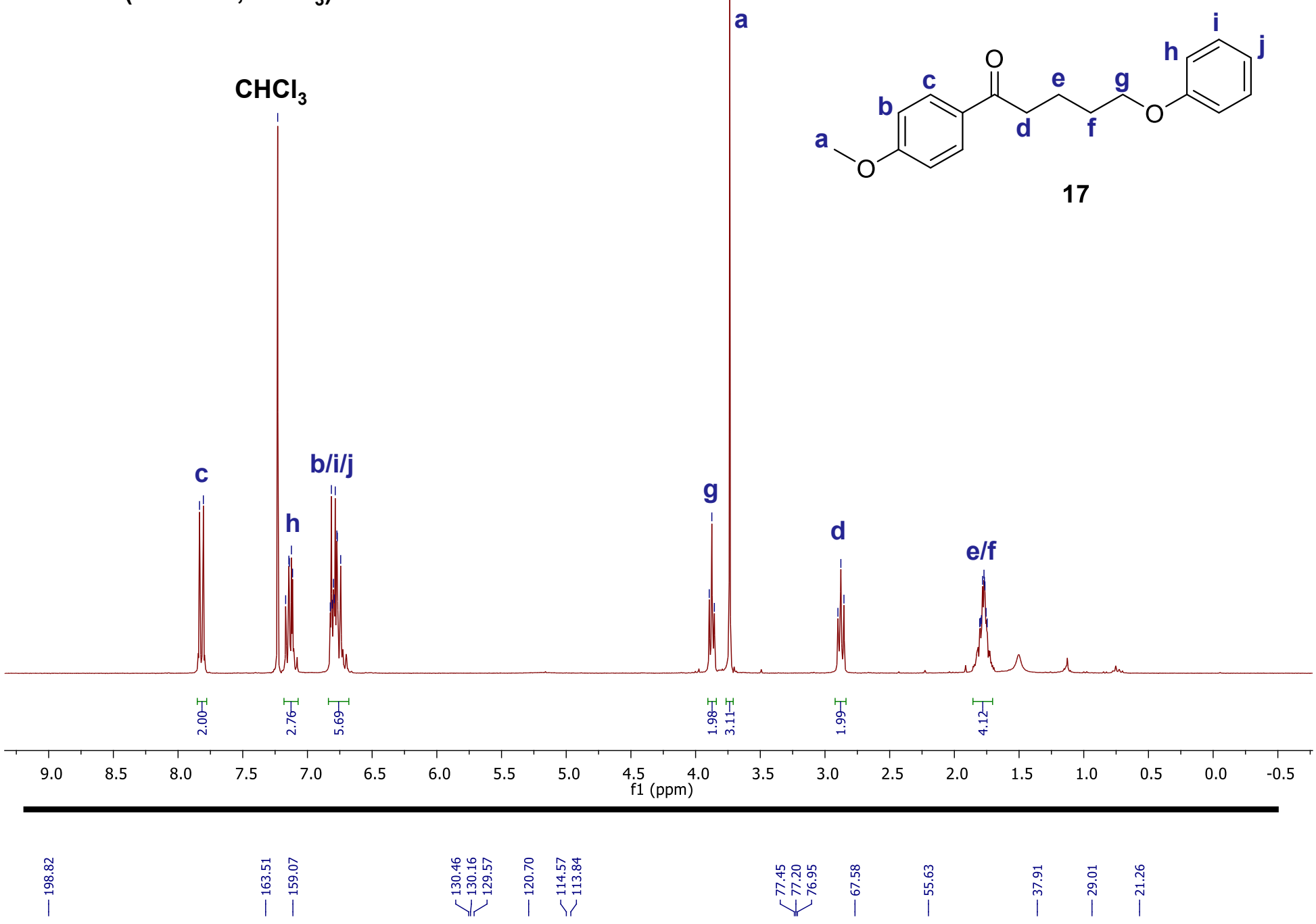

${ }^{13} \mathrm{C}-\mathrm{NMR}\left(126 \mathrm{MHz} \mathrm{CDCl}_{3}\right.$ )

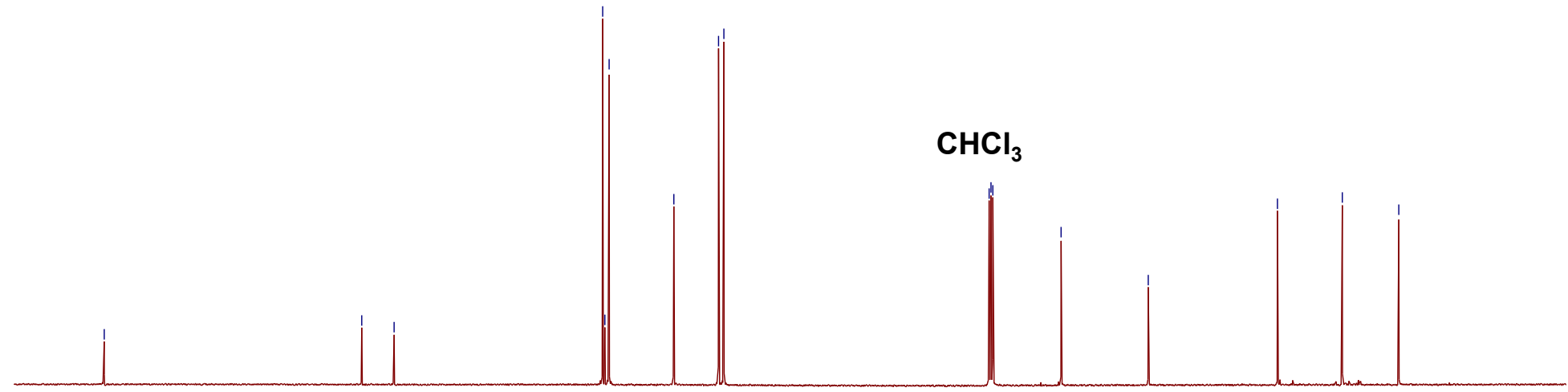


${ }^{1} \mathrm{H}-\mathrm{NMR}$ (300 MHz; $\mathrm{CDCl}_{3}$ )

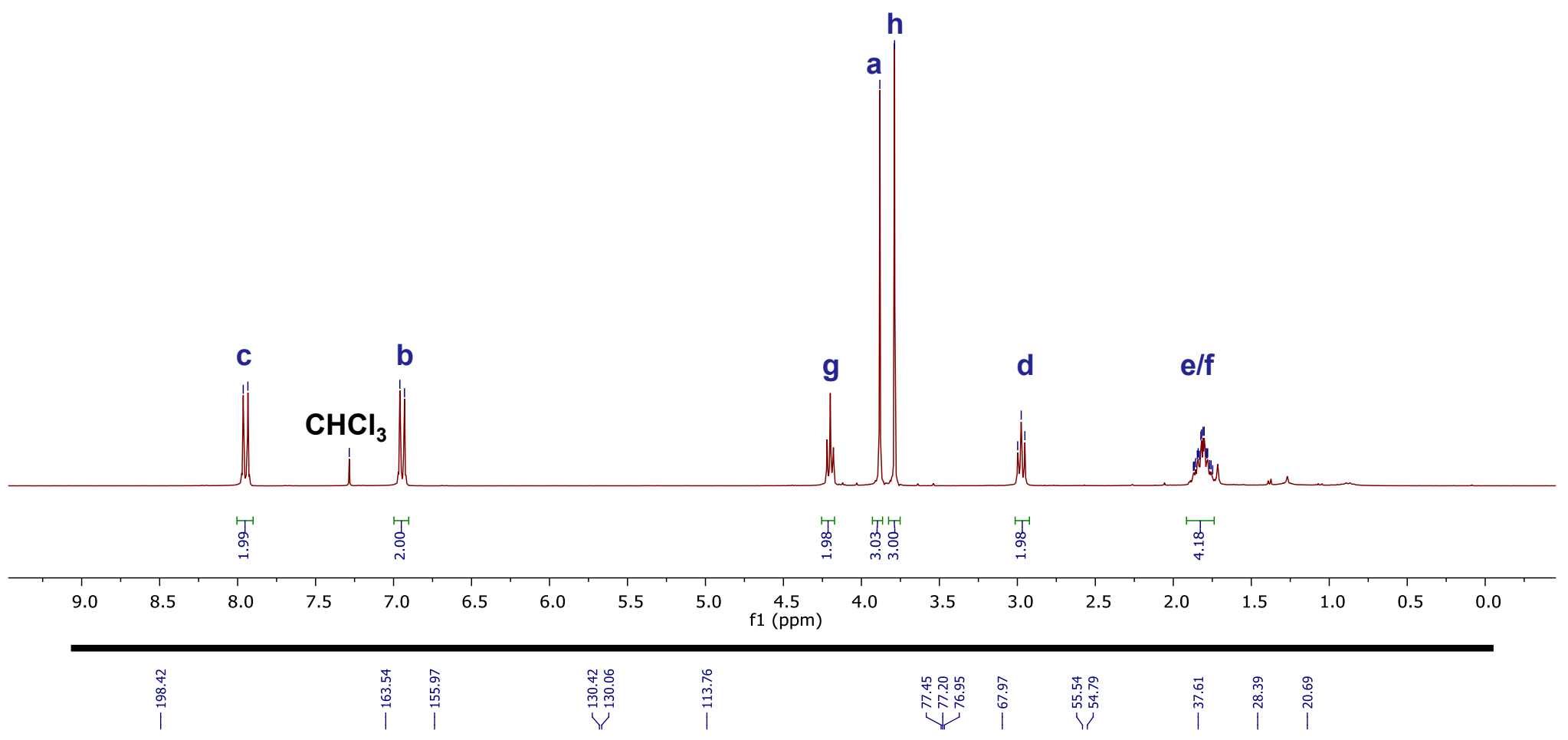

${ }^{13} \mathrm{C}-\mathrm{NMR}$ (126 MHz; $\mathrm{CDCl}_{3}$ )

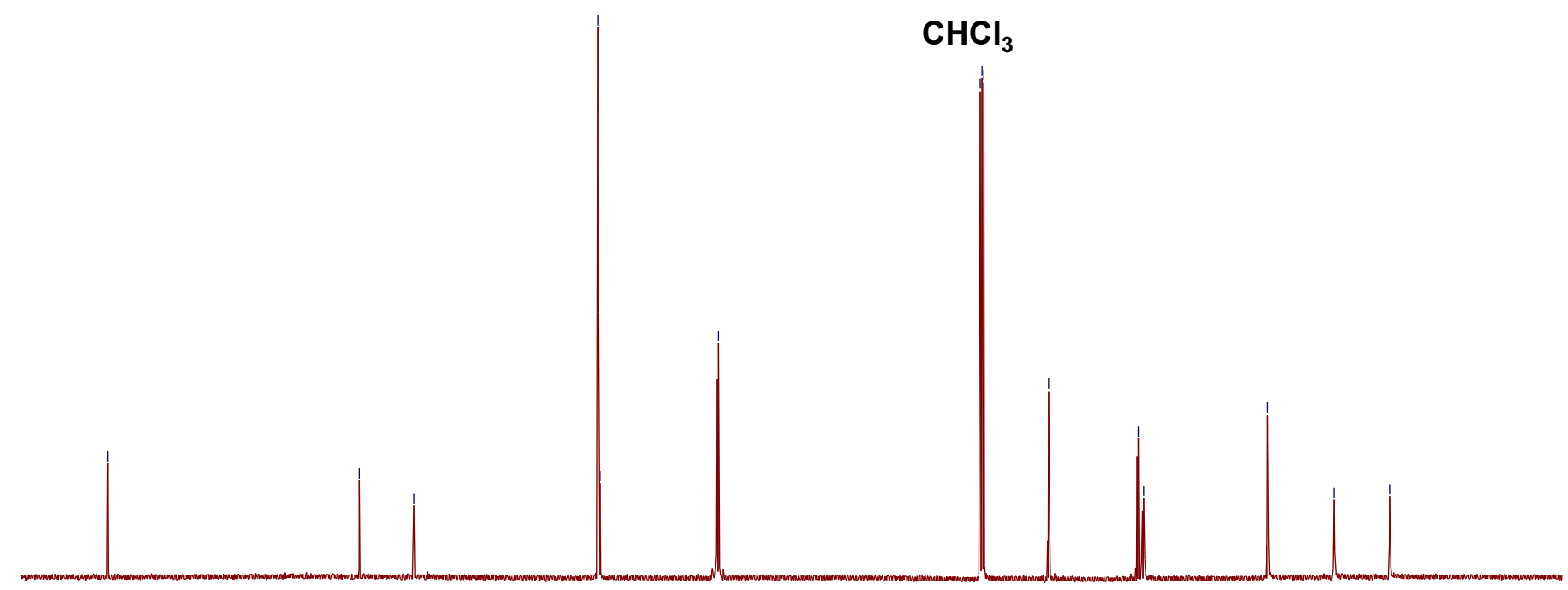

\begin{tabular}{|c|c|c|c|c|c|c|c|c|c|c|c|c|c|c|c|c|c|c|c|c|c|}
\hline$T$ & 1 & 1 & 1 & 1 & $T$ & 1 & 1 & 1 & 1 & 1 & 1 & 1 & 1 & 1 & $T$ & $T$ & 1 & 1 & $T$ & 1 & $T$ \\
\hline 210 & 200 & 190 & 180 & 170 & 160 & 150 & 140 & 130 & 120 & 110 & $\begin{array}{r}100 \\
\mathrm{ppm})\end{array}$ & 90 & 80 & 70 & 60 & 50 & 40 & 30 & 20 & 10 & 0 \\
\hline
\end{tabular}


${ }^{1} \mathrm{H}-\mathrm{NMR}$ (300 MHz; $\mathrm{CDCl}_{3}$ )

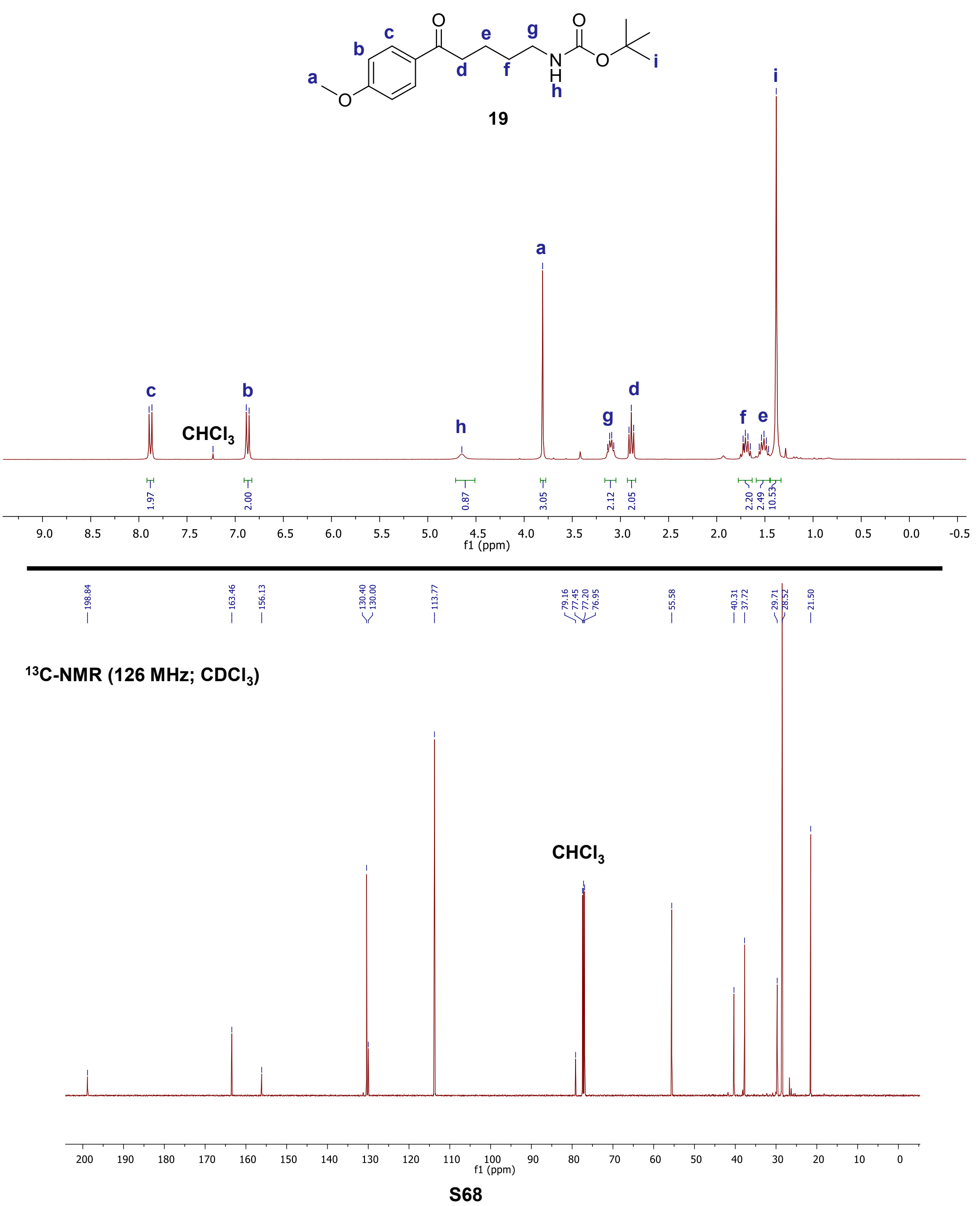


${ }^{1} \mathrm{H}-\mathrm{NMR}\left(300 \mathrm{MHz} ; \mathrm{CDCl}_{3}\right.$ )

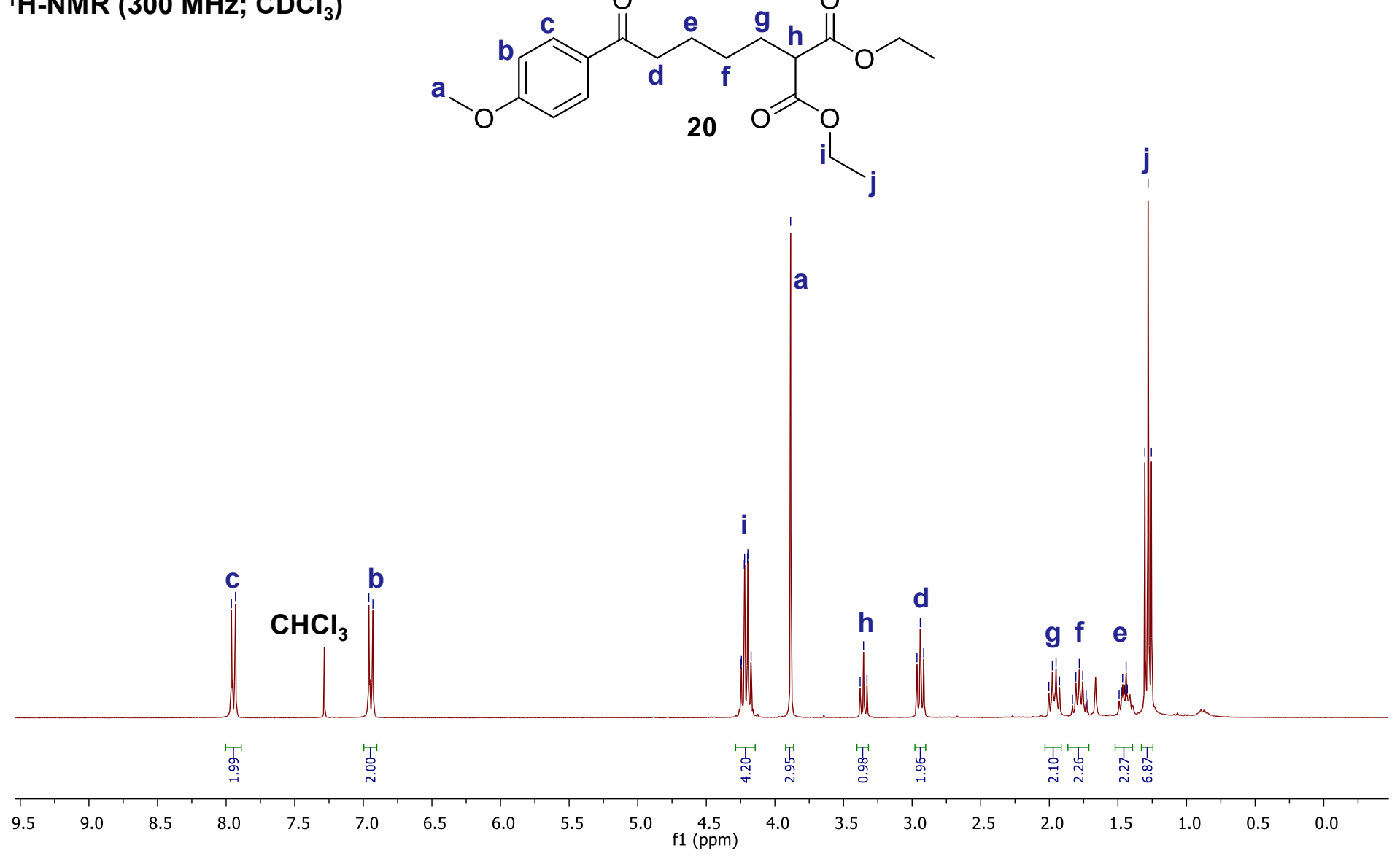

\begin{tabular}{|c|c|c|c|c|c|c|c|}
\hline $\begin{array}{l}\hat{0} \\
\infty \\
\stackrel{1}{\mid}\end{array}$ & 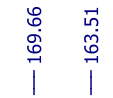 & 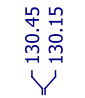 & 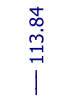 & 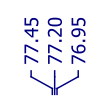 & 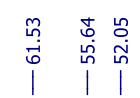 & $\begin{array}{l}\hat{a} \\
\hat{m} \\
1\end{array}$ & 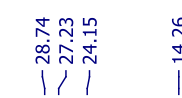 \\
\hline
\end{tabular}

${ }^{13} \mathrm{C}-\mathrm{NMR}$ (126 MHz; $\mathrm{CDCl}_{3}$ )

\section{$\mathrm{CHCl}_{3}$}

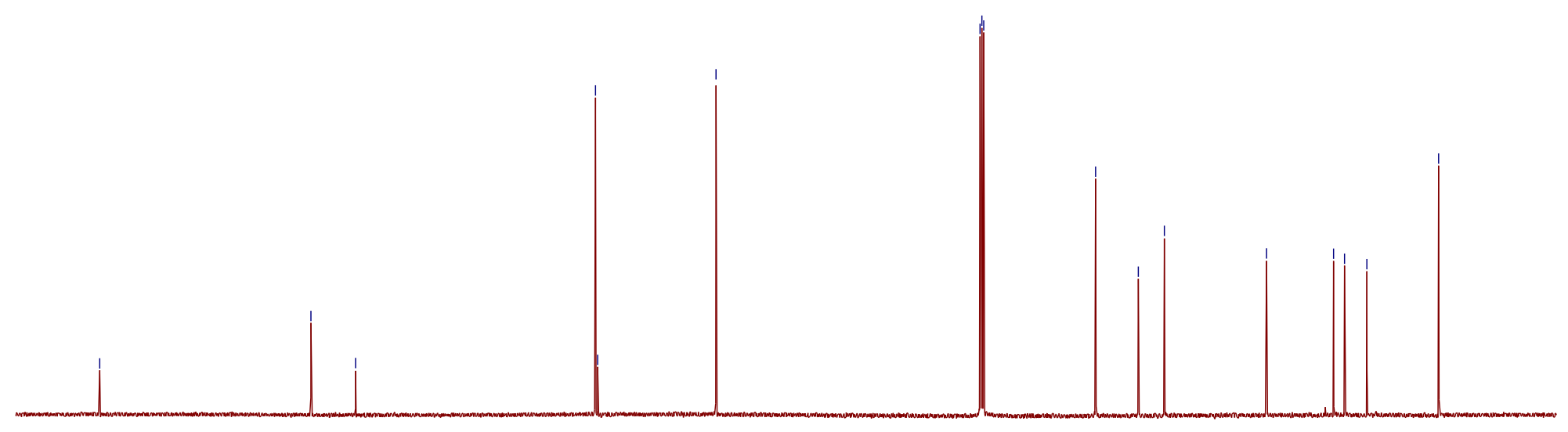

\begin{tabular}{|c|c|c|c|c|c|c|c|c|c|c|c|c|c|c|c|c|c|c|c|c|c|}
\hline T & $T$ & $T$ & $T$ & $T$ & 1 & $T$ & $T$ & 7 & $T$ & $T$ & $T$ & $T$ & T & 7 & $T$ & $T$ & $T$ & $T$ & $T$ & $T$ & $T$ \\
\hline 210 & 200 & 190 & 180 & 170 & 160 & 150 & 140 & 130 & 120 & $\begin{array}{r}110 \\
\mathrm{f} 1\end{array}$ & $\begin{array}{c}100 \\
\mathrm{pm})\end{array}$ & 90 & 80 & 70 & 60 & 50 & 40 & 30 & 20 & 10 & 0 \\
\hline
\end{tabular}


${ }^{1} \mathrm{H}-\mathrm{NMR}$ (300 MHz; $\mathrm{CDCl}_{3}$ )

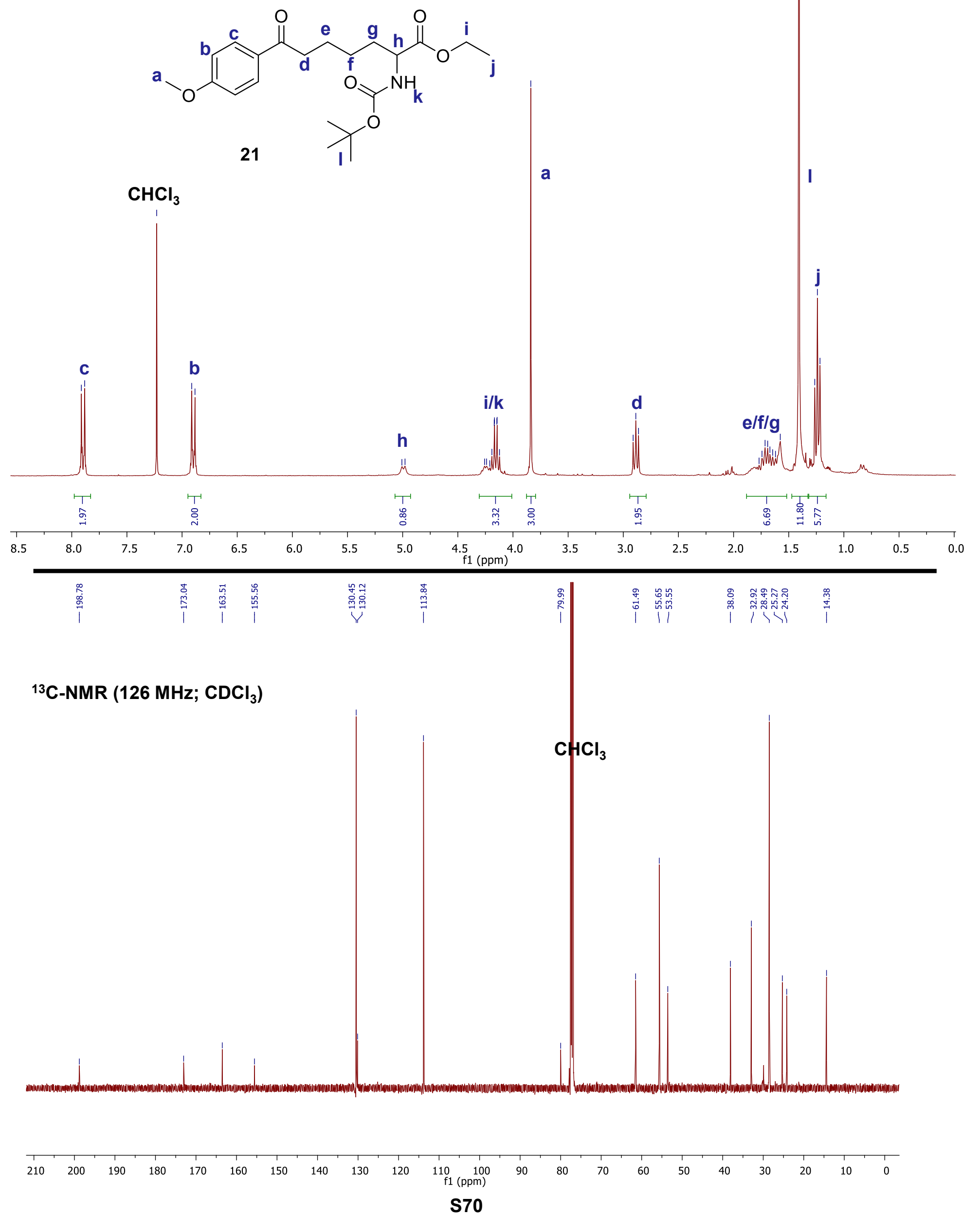


${ }^{1} \mathrm{H}-\mathrm{NMR}$ (300 MHz; $\mathrm{CDCl}_{3}$ )

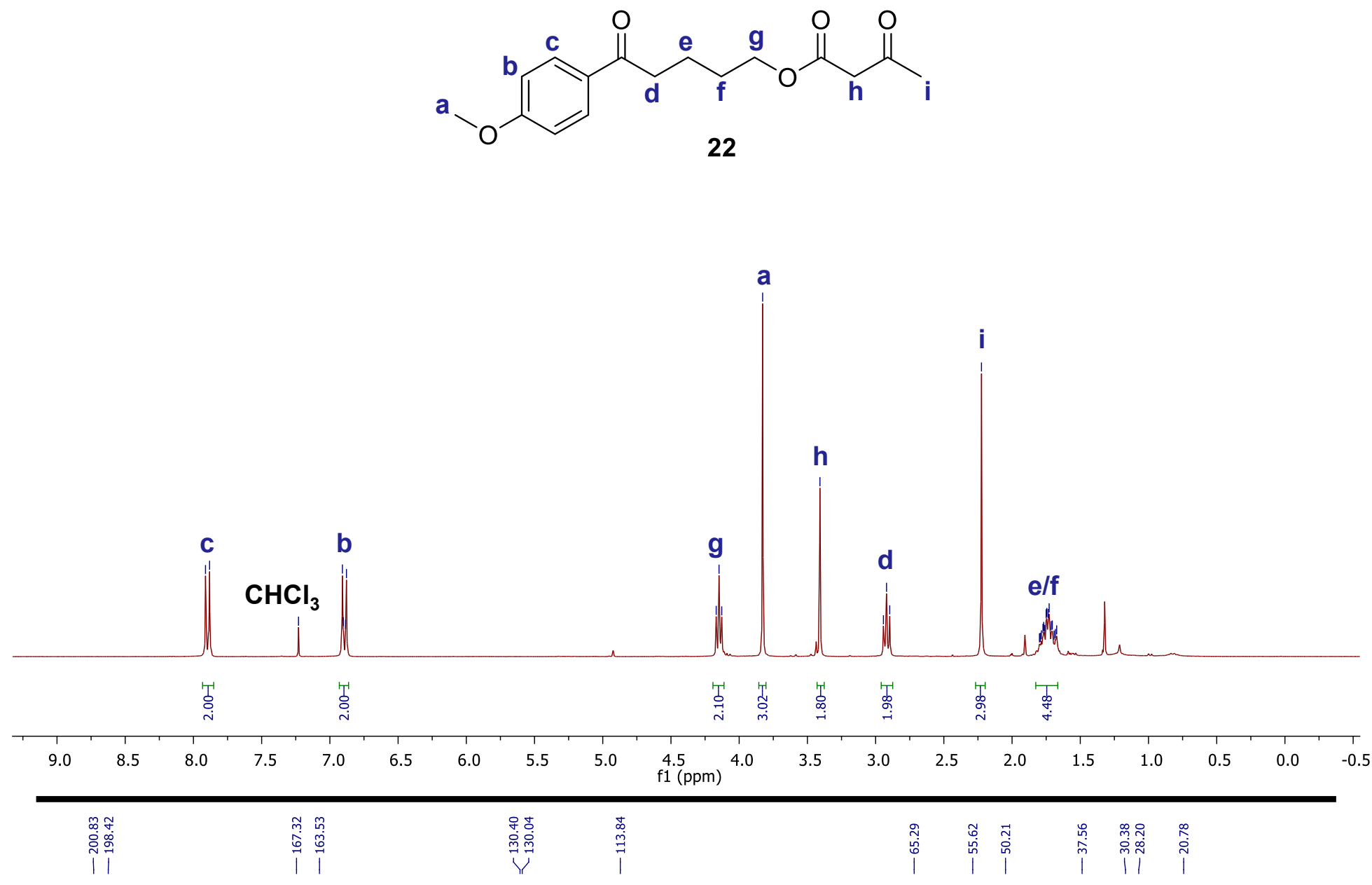

${ }^{13} \mathrm{C}-\mathrm{NMR}$ (126 MHz; $\mathrm{CDCl}_{3}$ )

$\mathrm{CHCl}_{3}$

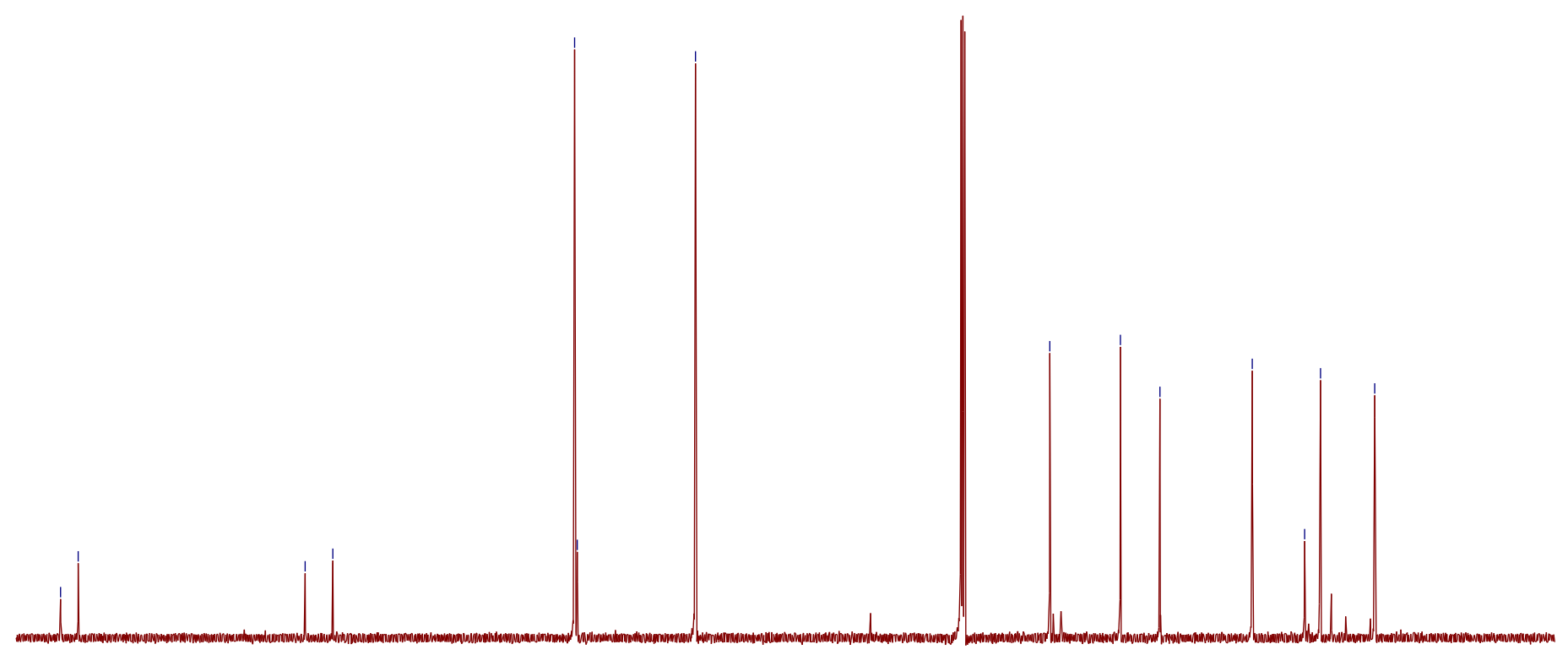

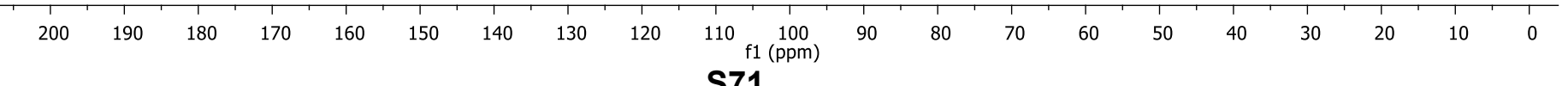


${ }^{1} \mathrm{H}-\mathrm{NMR}\left(300 \mathrm{MHz}^{-\mathrm{CDCl}_{3}}\right.$ )<smiles>[Y]c1csc(C(C)NC(=O)O[CH]C(F)CC(=O)c2ccc(OC)cc2)c1</smiles>

23

a
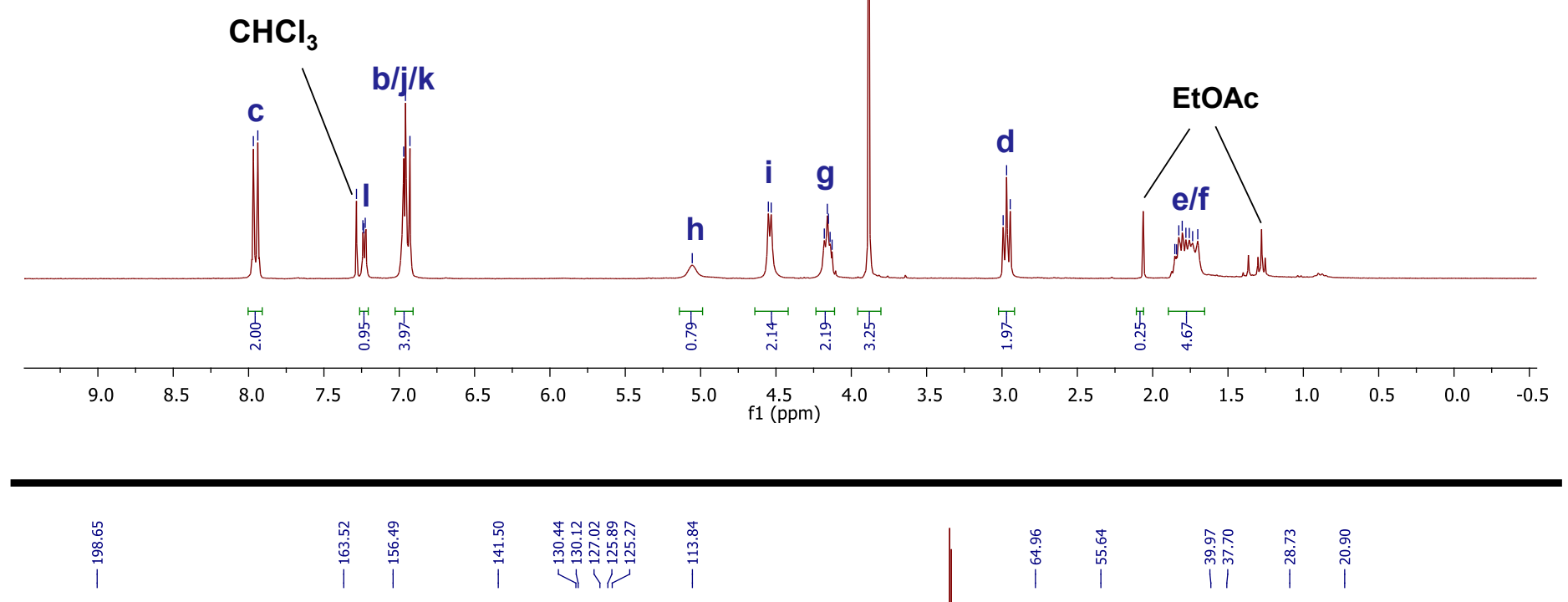

${ }^{13} \mathrm{C}-\mathrm{NMR}\left(126 \mathrm{MHz}^{\mathrm{CDCl}_{3}}\right)$

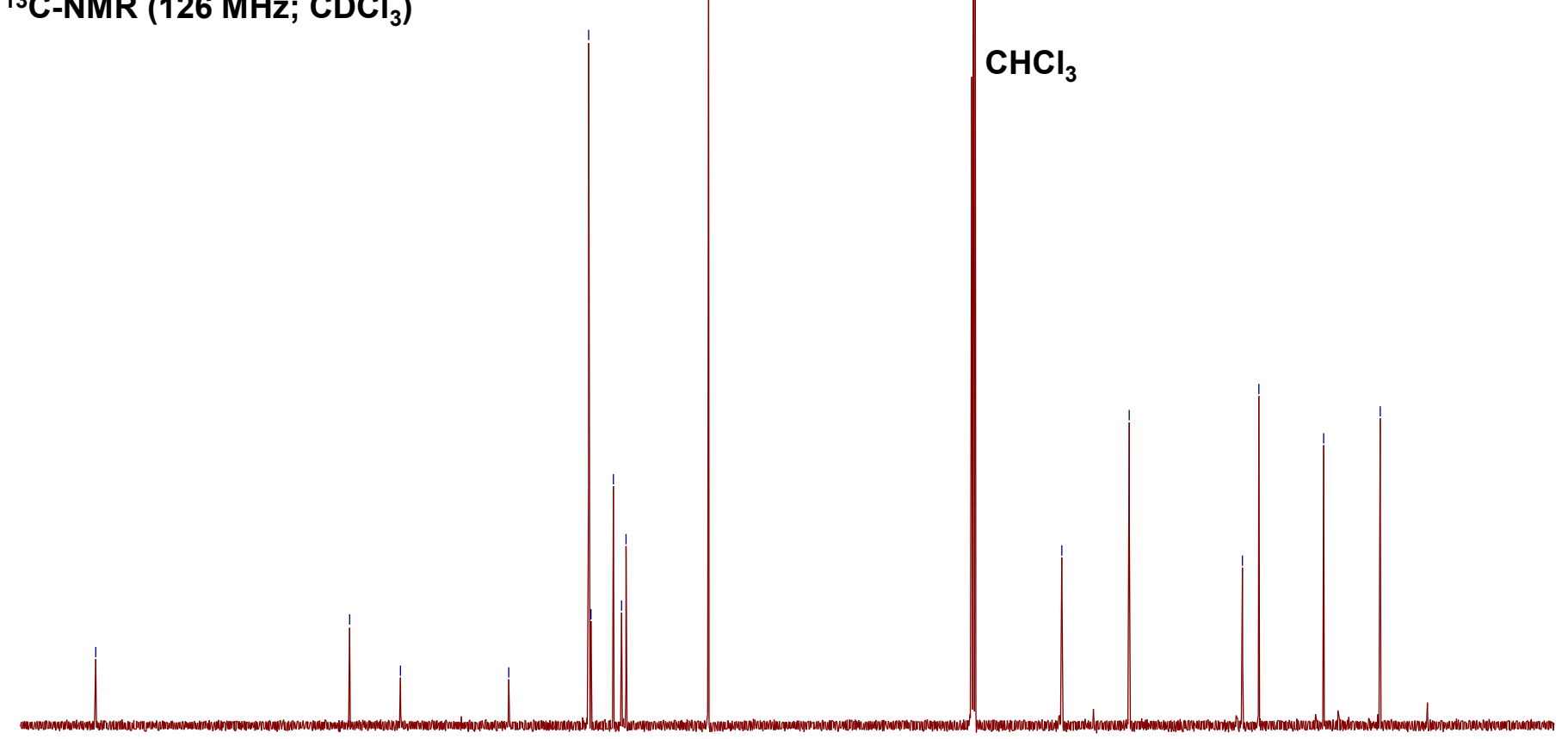

$\begin{array}{llllllllll}200 & 190 & 180 & 170 & 160 & 150 & 140 & 130 & 120 & 110 \begin{array}{r}100 \\ \mathrm{f} 1(\mathrm{ppm})\end{array}\end{array}$ 
${ }^{1} \mathrm{H}-\mathrm{NMR}\left(300 \mathrm{MHz} ; \mathrm{CDCl}_{3}\right.$ )<smiles>[X]c1coc(C(C)NC(=O)OCC(F)CCC(=O)c2ccc(O[2H])cc2)c1</smiles>

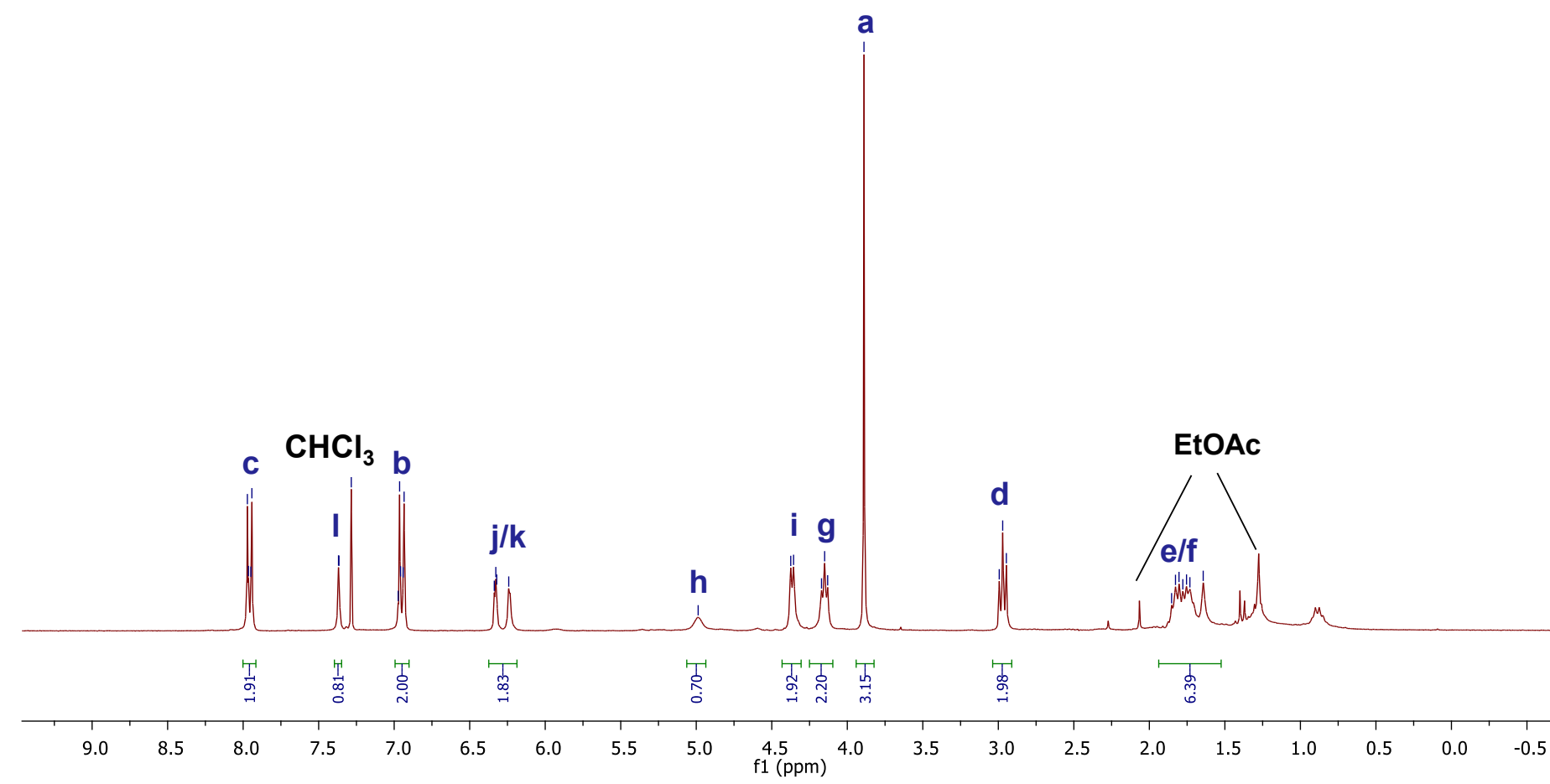

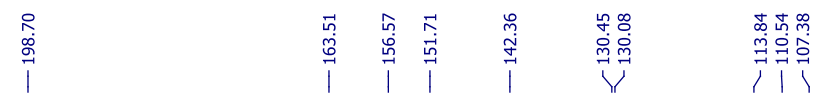

${ }^{13} \mathrm{C}-\mathrm{NMR}\left(126 \mathrm{MHz} ; \mathrm{CDCl}_{3}\right.$ )

$\mathrm{CHCl}_{3}$

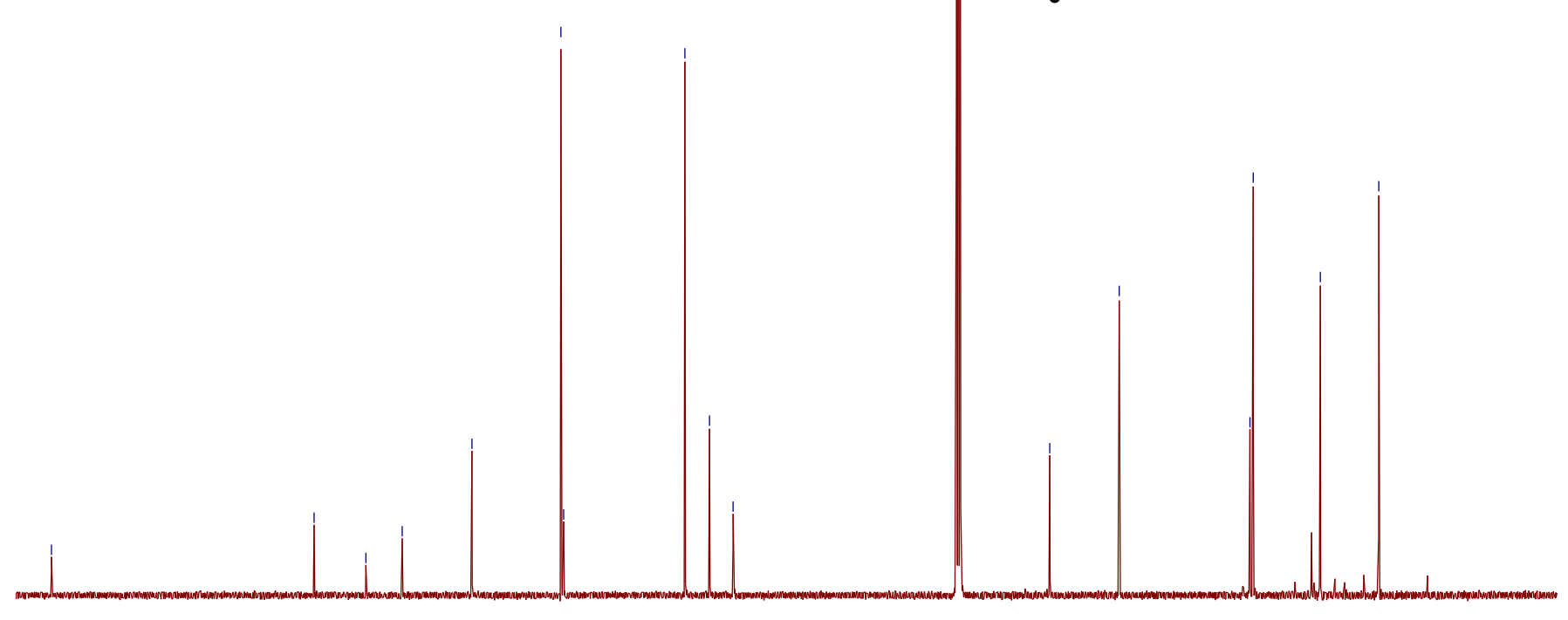


${ }^{1} \mathrm{H}-\mathrm{NMR}\left(300 \mathrm{MHz}^{\mathrm{CDCl}}{ }_{3}\right)$

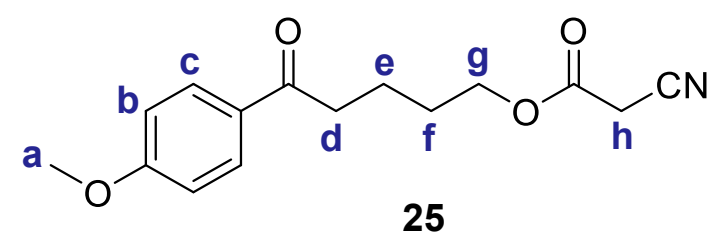

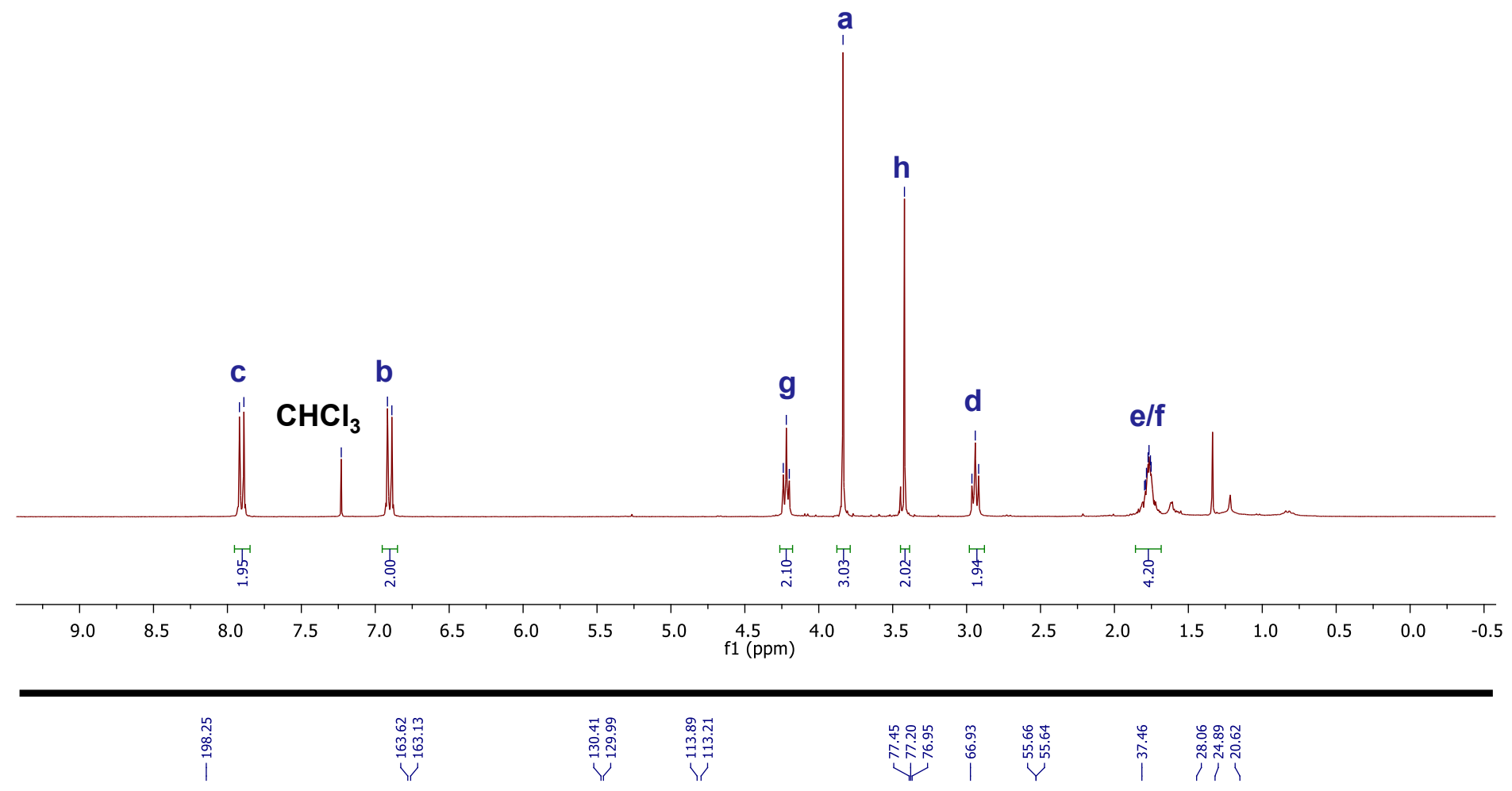

${ }^{13} \mathrm{C}-\mathrm{NMR}\left(126 \mathrm{MHz}^{\mathrm{CDCl}}{ }_{3}\right)$

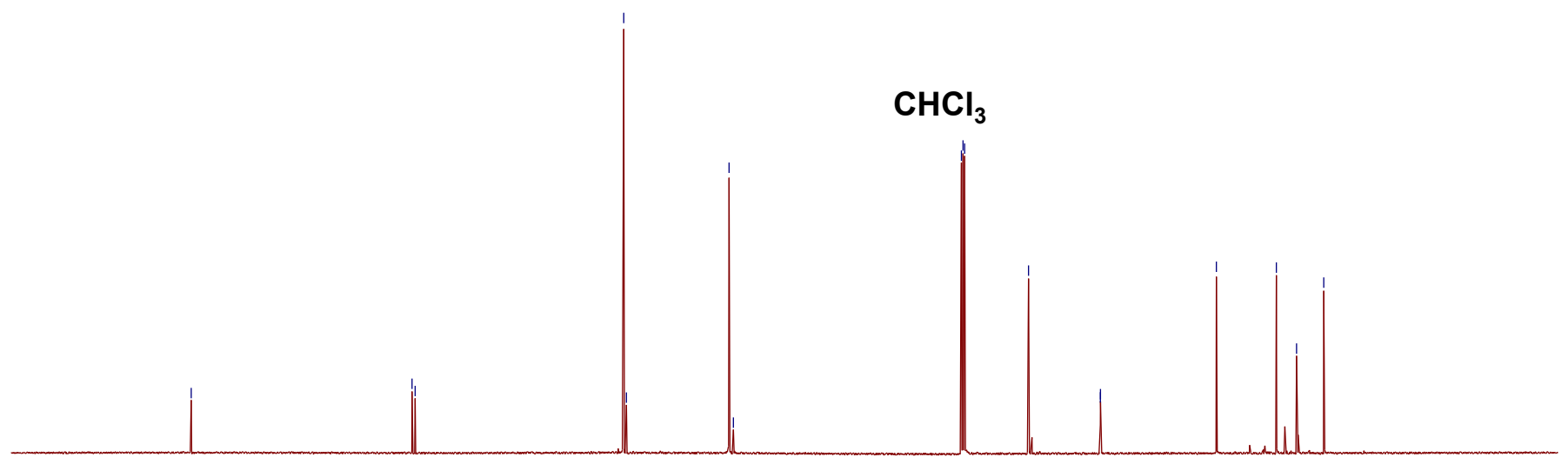

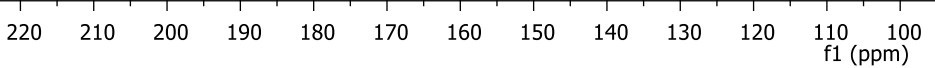


${ }^{1} \mathrm{H}-\mathrm{NMR}$ (300 MHz; $\mathrm{CDCl}_{3}$ )

a

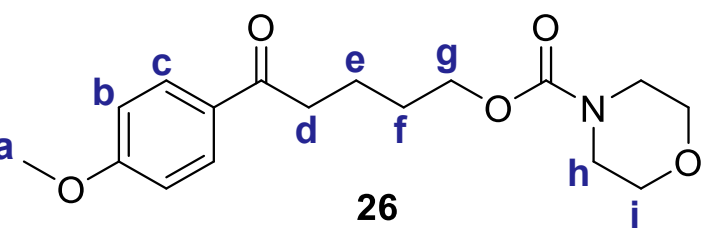

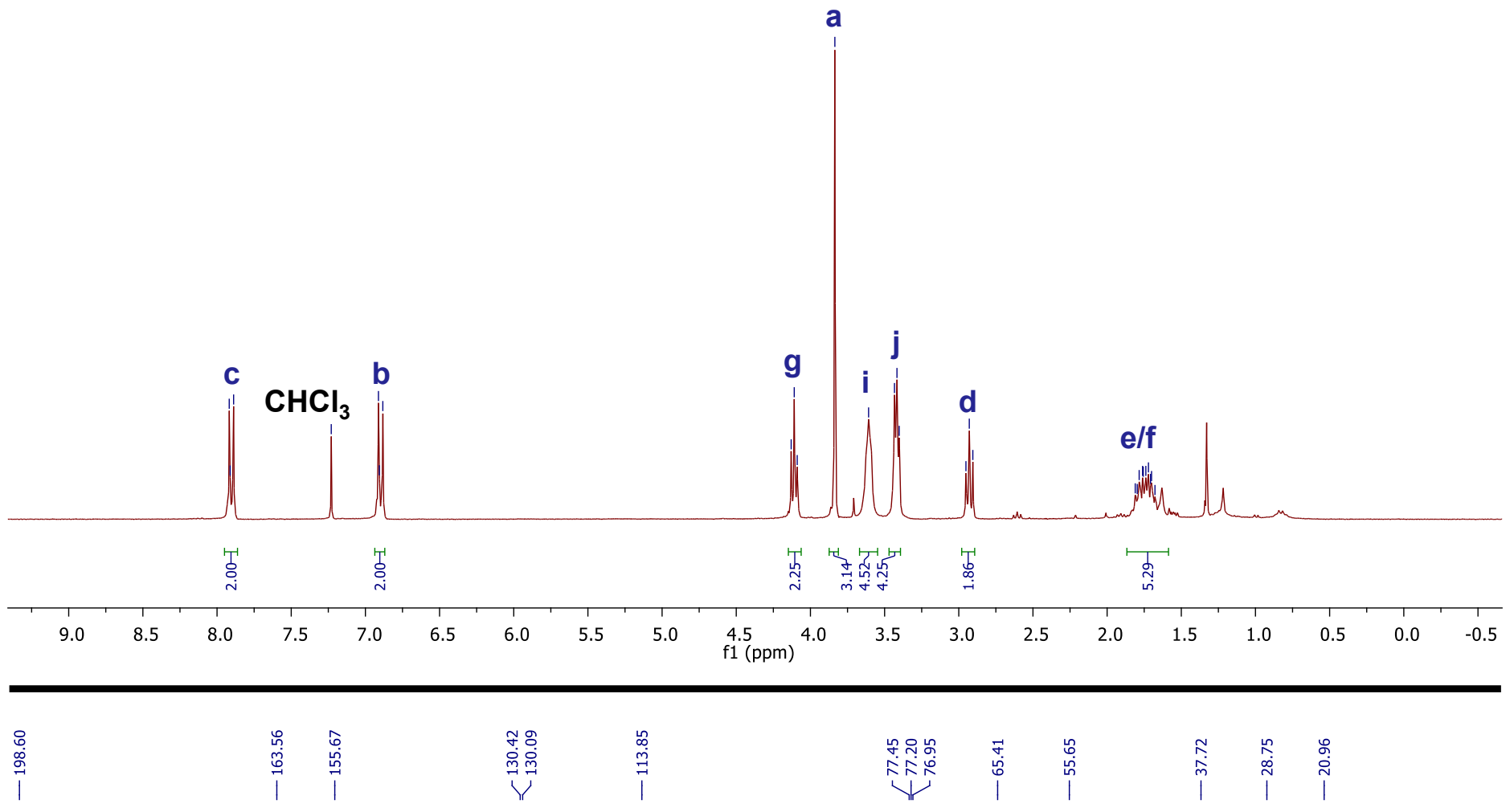

${ }^{13} \mathrm{C}-\mathrm{NMR}\left(126 \mathrm{MHz} ; \mathrm{CDCl}_{3}\right.$ )

\section{$\mathrm{CHCl}_{3}$}

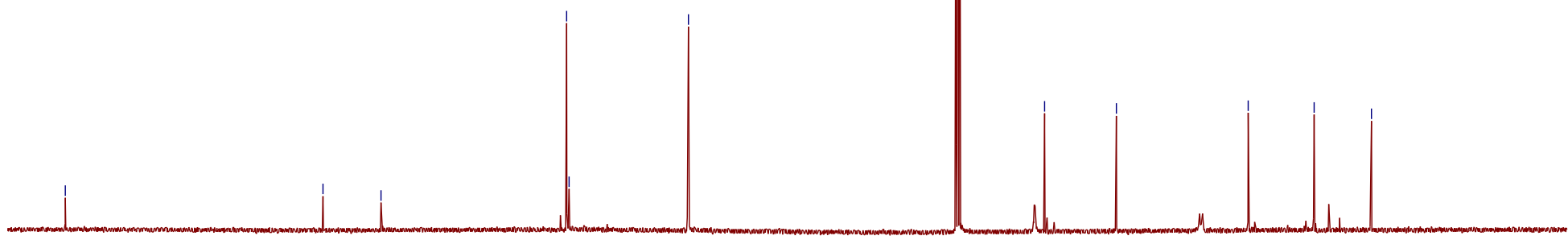

\begin{tabular}{|c|c|c|c|c|c|c|c|c|c|c|c|c|c|c|c|c|c|c|c|c|}
\hline 200 & 190 & 180 & 170 & 160 & 150 & 140 & 130 & 120 & 110 & $\begin{array}{c}100 \\
\mathrm{f} 1(\mathrm{ppm})\end{array}$ & 90 & 80 & 70 & 60 & 50 & 40 & 30 & 20 & 10 & 0 \\
\hline
\end{tabular}


${ }^{1} \mathrm{H}-\mathrm{NMR}\left(300 \mathrm{MHz} ; \mathrm{CDCl}_{3}\right.$ )

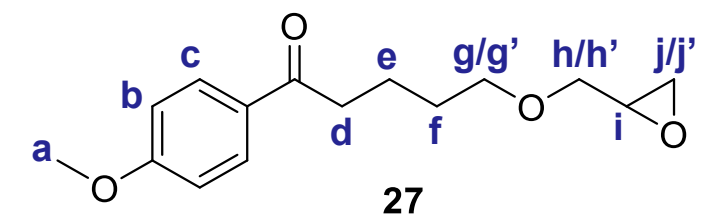

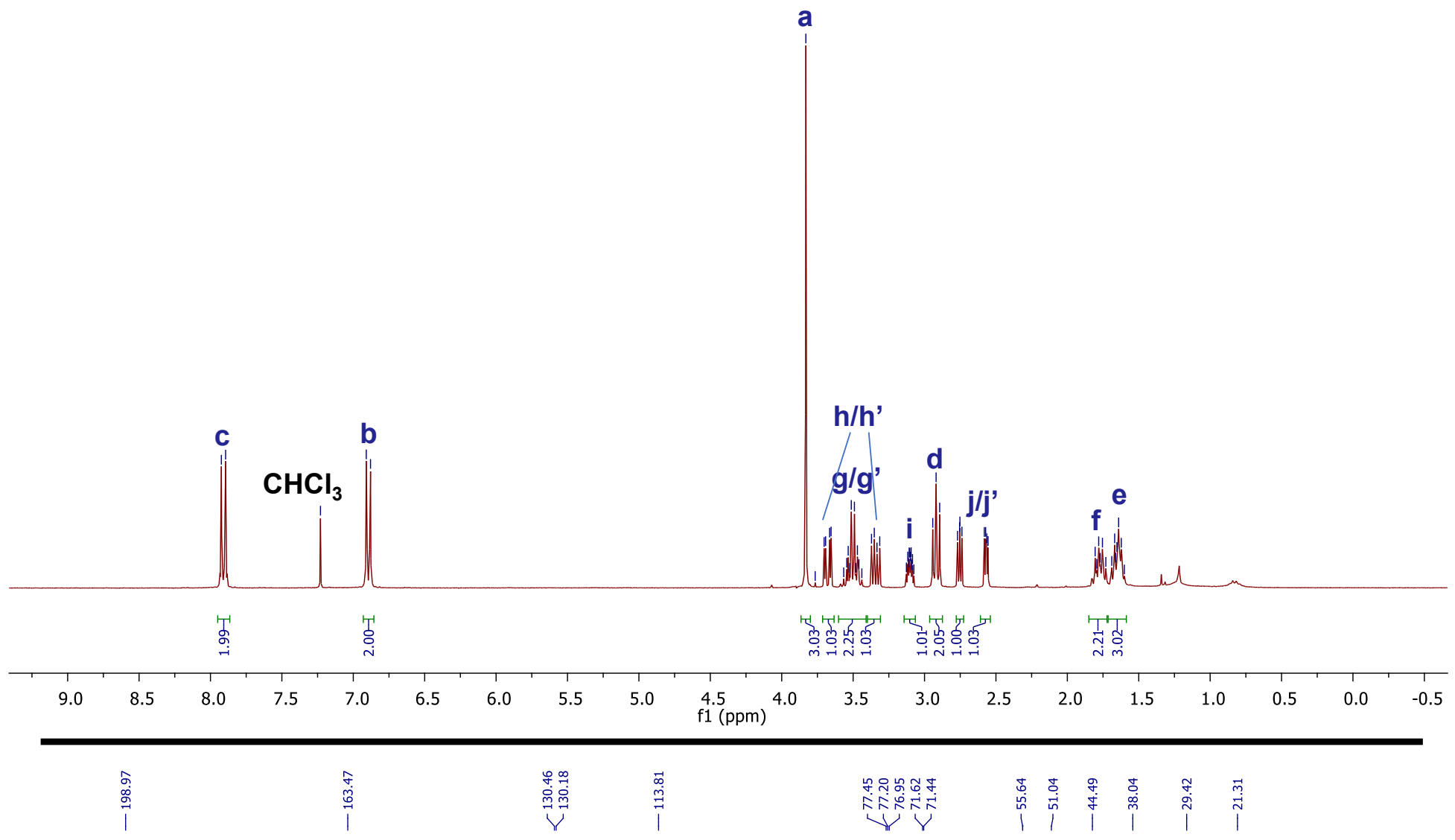

${ }^{13} \mathrm{C}-\mathrm{NMR}\left(126 \mathrm{MHz}^{\mathrm{CDCl}}{ }_{3}\right)$

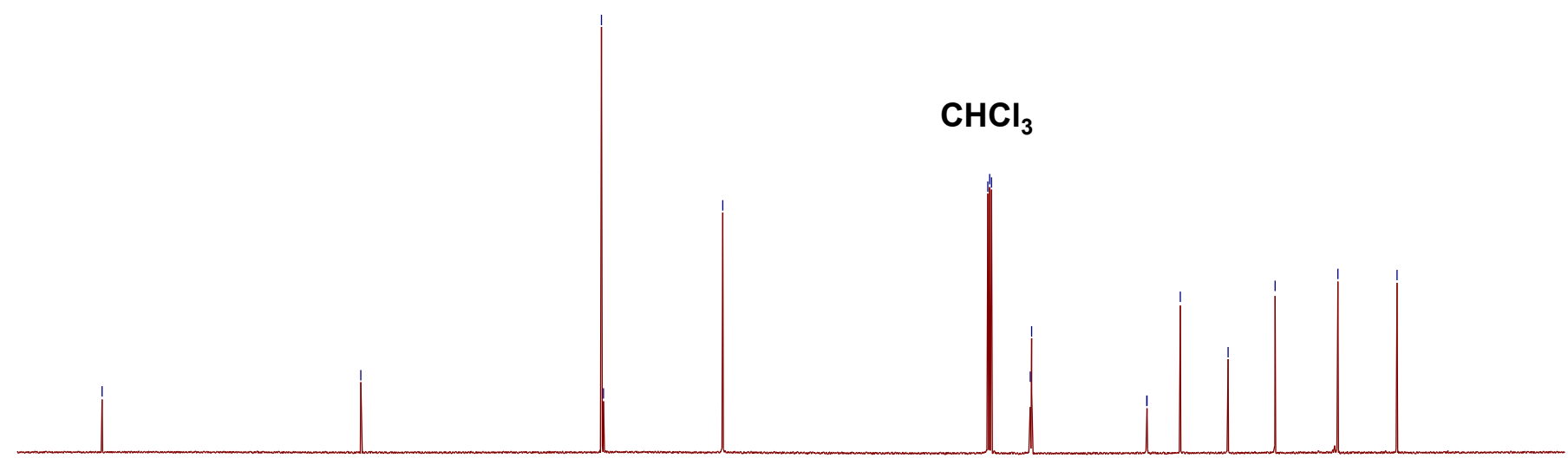

\begin{tabular}{|c|c|c|c|c|c|c|c|c|c|c|c|c|c|c|c|c|c|c|c|c|}
\hline$T$ & 1 & 1 & 1 & 1 & 1 & 1 & 1 & $T$ & $T$ & 1 & $T$ & 1 & 1 & $T$ & $T$ & 1 & $T$ & 1 & 1 & 1 \\
\hline 210 & 200 & 190 & 180 & 170 & 160 & 150 & 140 & 130 & 120 & $\begin{array}{r}110 \\
\mathrm{f} 1\end{array}$ & $\begin{array}{l}100 \\
\mathrm{pm})\end{array}$ & 90 & 80 & 70 & 60 & 50 & 40 & 30 & 20 & 10 \\
\hline
\end{tabular}


${ }^{1} \mathrm{H}-\mathrm{NMR}\left(300 \mathrm{MHz} ; \mathrm{CDCl}_{3}\right.$ )

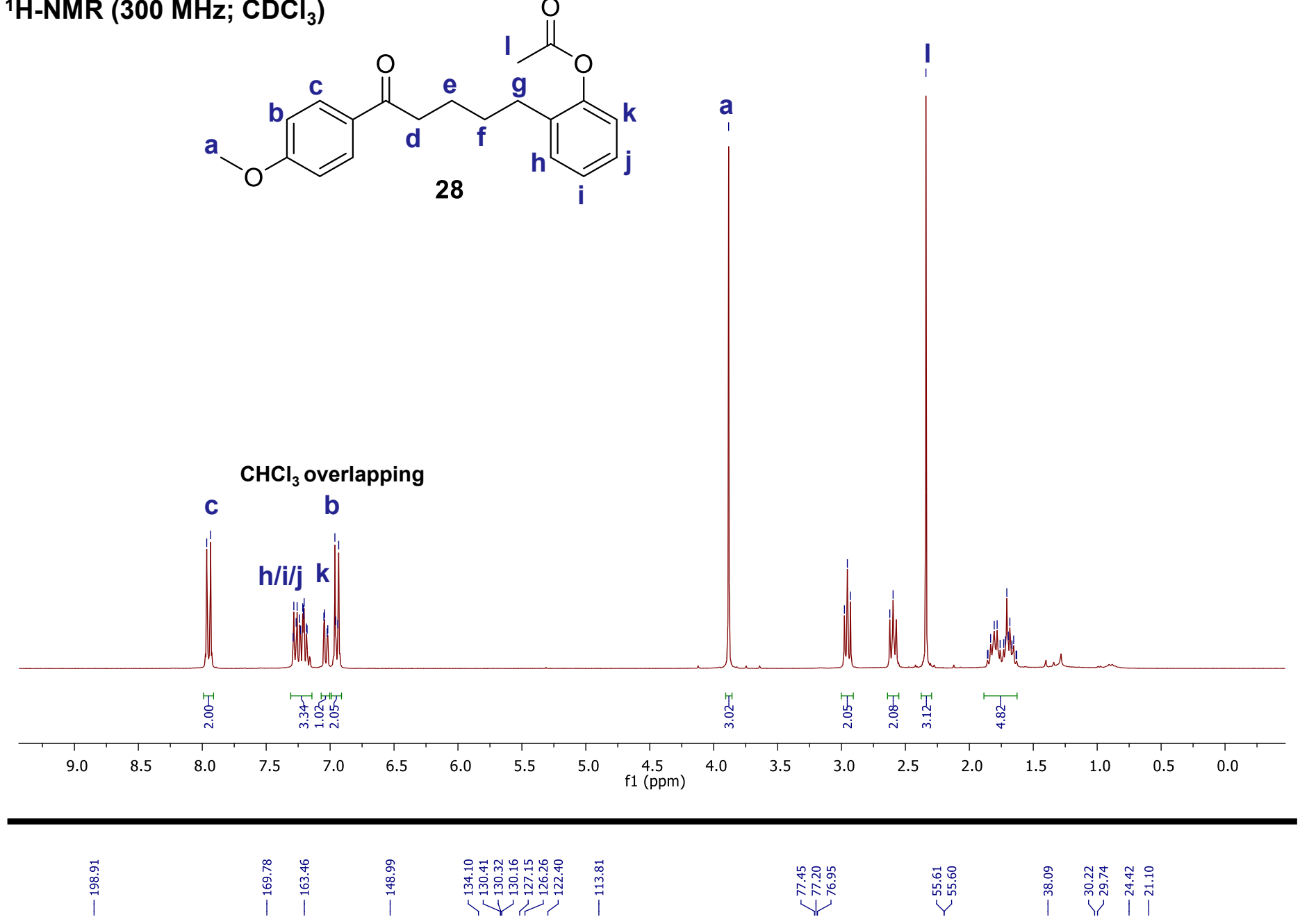

${ }^{13} \mathrm{C}-\mathrm{NMR}\left(126 \mathrm{MHz} ; \mathrm{CDCl}_{3}\right.$ )
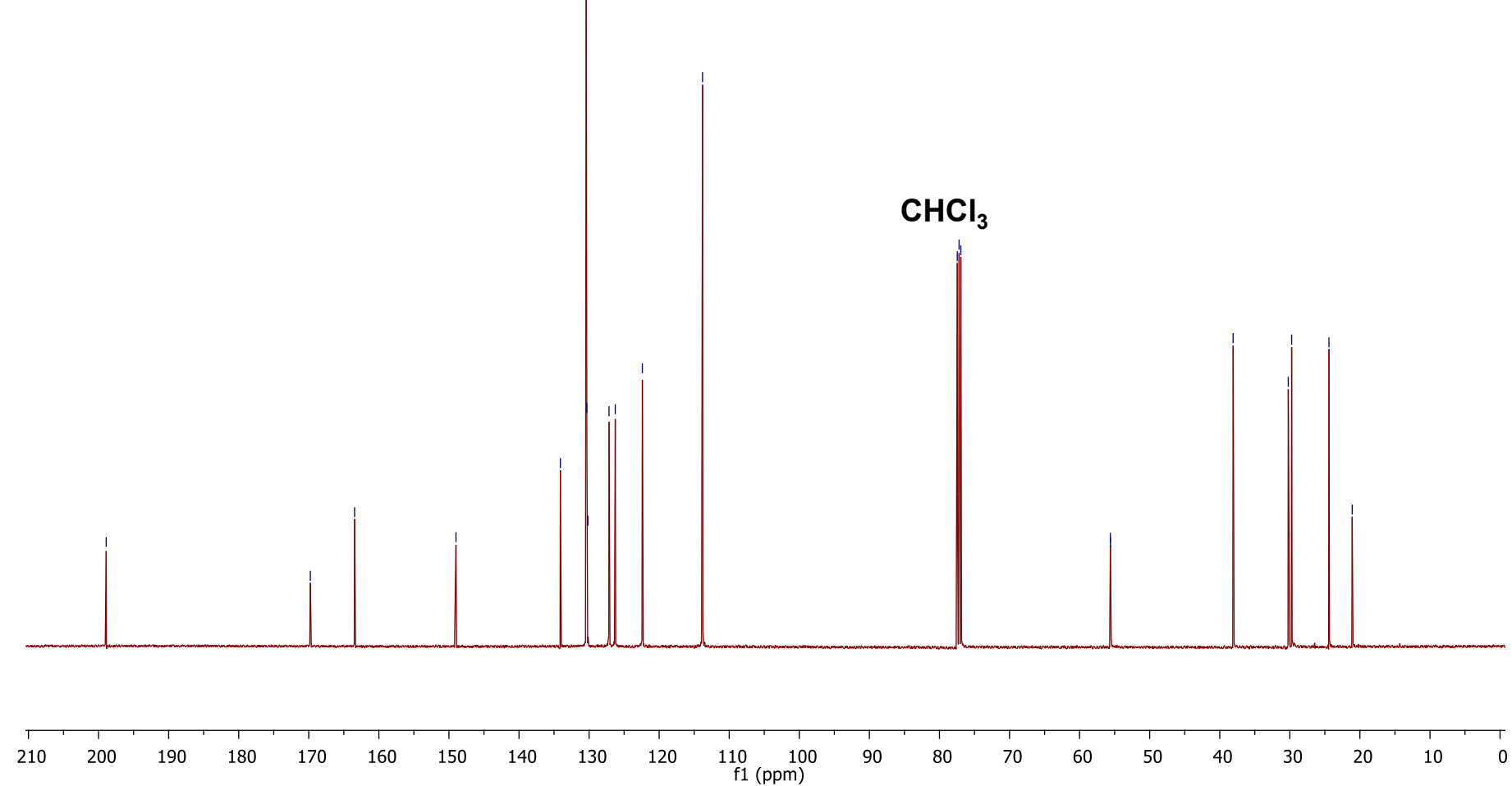
${ }^{1} \mathrm{H}-\mathrm{NMR}\left(300 \mathrm{MHz} ; \mathrm{CDCl}_{3}\right.$ )<smiles>[X]c1cc(I)cc(CC(C)CC(=O)c2ccc(OC)cc2)c1OC</smiles>

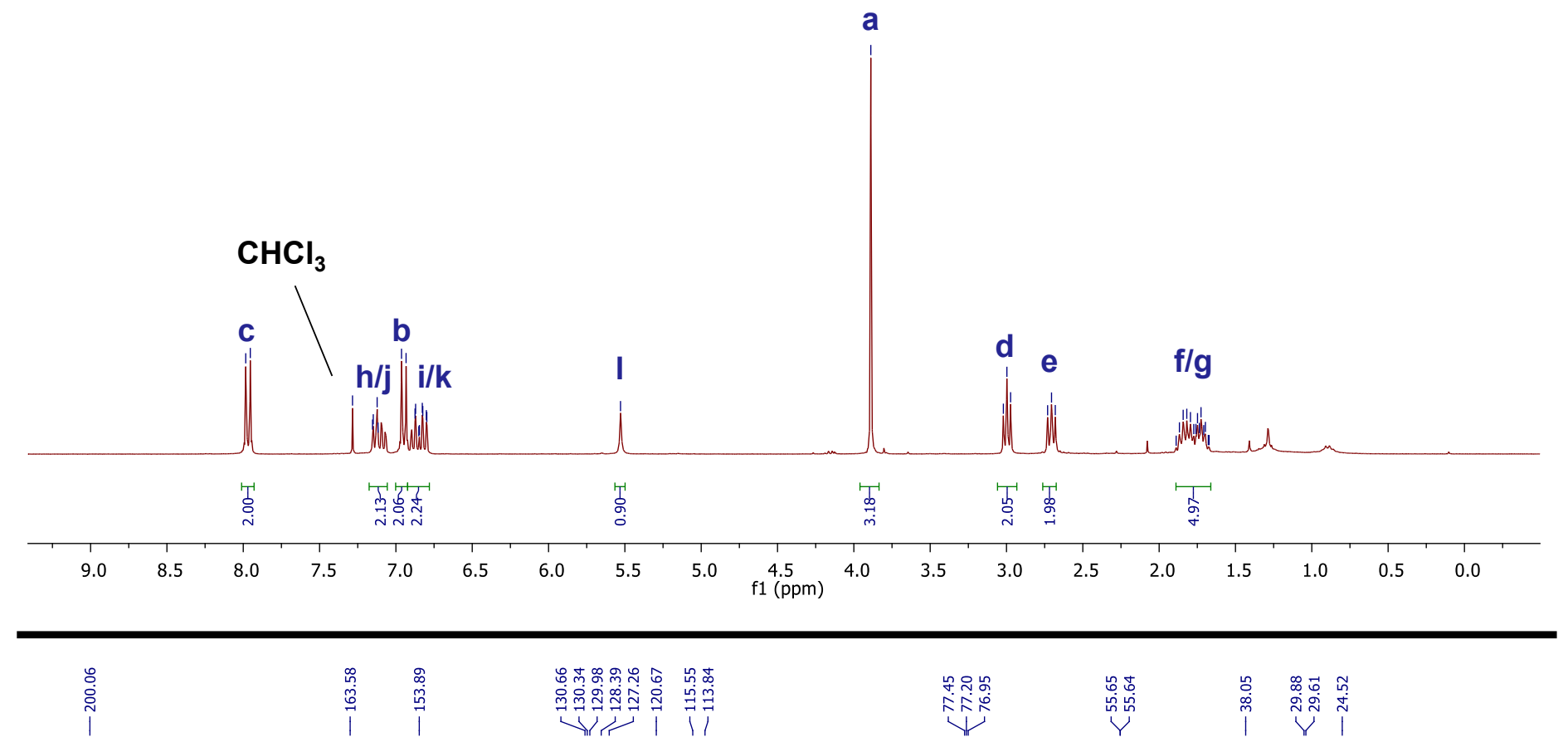

${ }^{13} \mathrm{C}-\mathrm{NMR}\left(126 \mathrm{MHz} ; \mathrm{CDCl}_{3}\right.$ )

\section{$\mathrm{CHCl}_{3}$}

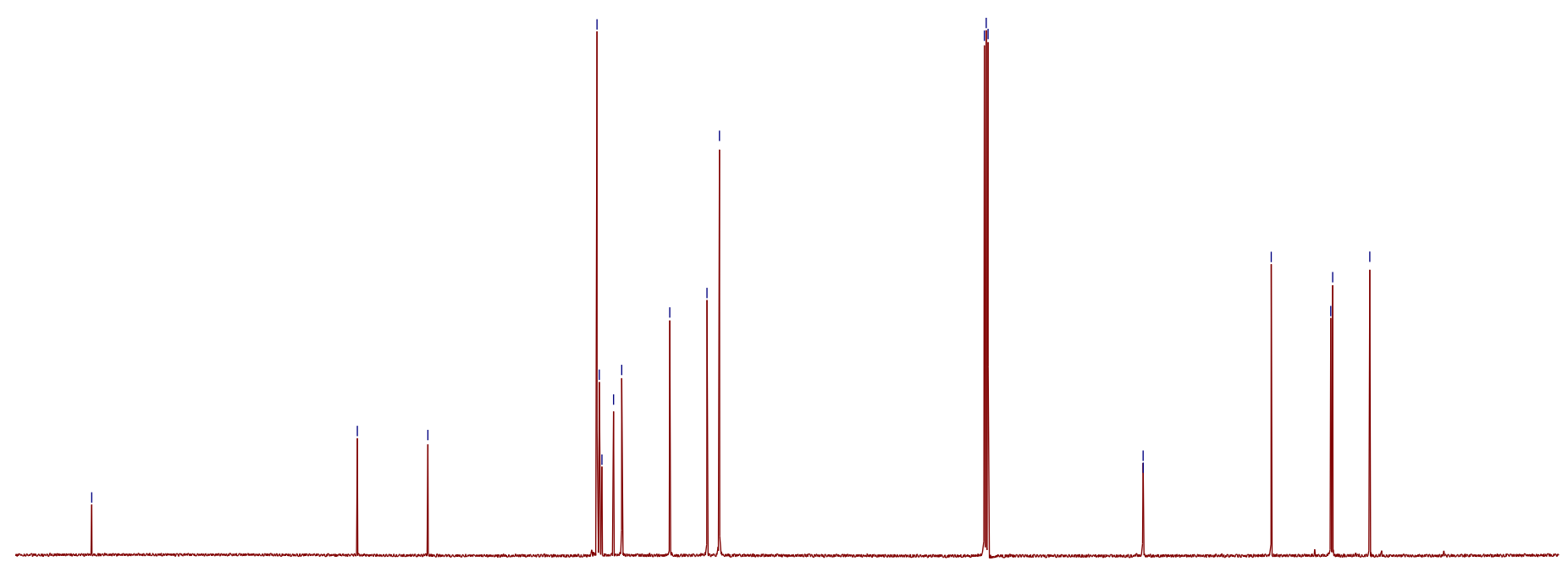

\begin{tabular}{|c|c|c|c|c|c|c|c|c|c|c|c|c|c|c|c|c|c|c|c|c|c|}
\hline$T$ & 1 & 1 & 1 & 1 & 1 & 1 & 1 & 1 & 1 & 1 & 1 & 1 & 1 & 1 & 1 & 1 & 1 & 1 & 1 & 1 & $T$ \\
\hline 210 & 200 & 190 & 180 & 170 & 160 & 150 & 140 & 130 & 120 & $\begin{array}{r}110 \\
\mathrm{f} 1\end{array}$ & $\begin{array}{c}100 \\
\text { pm) }\end{array}$ & 90 & 80 & 70 & 60 & 50 & 40 & 30 & 20 & 10 & 0 \\
\hline
\end{tabular}


${ }^{1} \mathrm{H}-\mathrm{NMR}\left(300 \mathrm{MHz} ; \mathrm{CDCl}_{3}\right.$ )

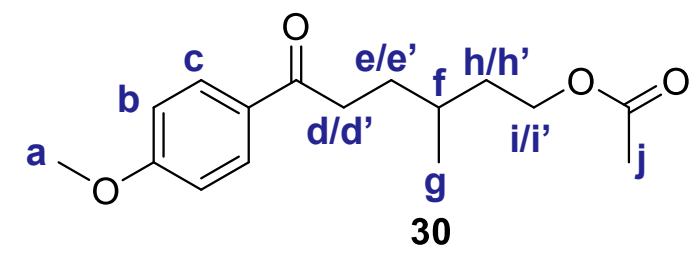

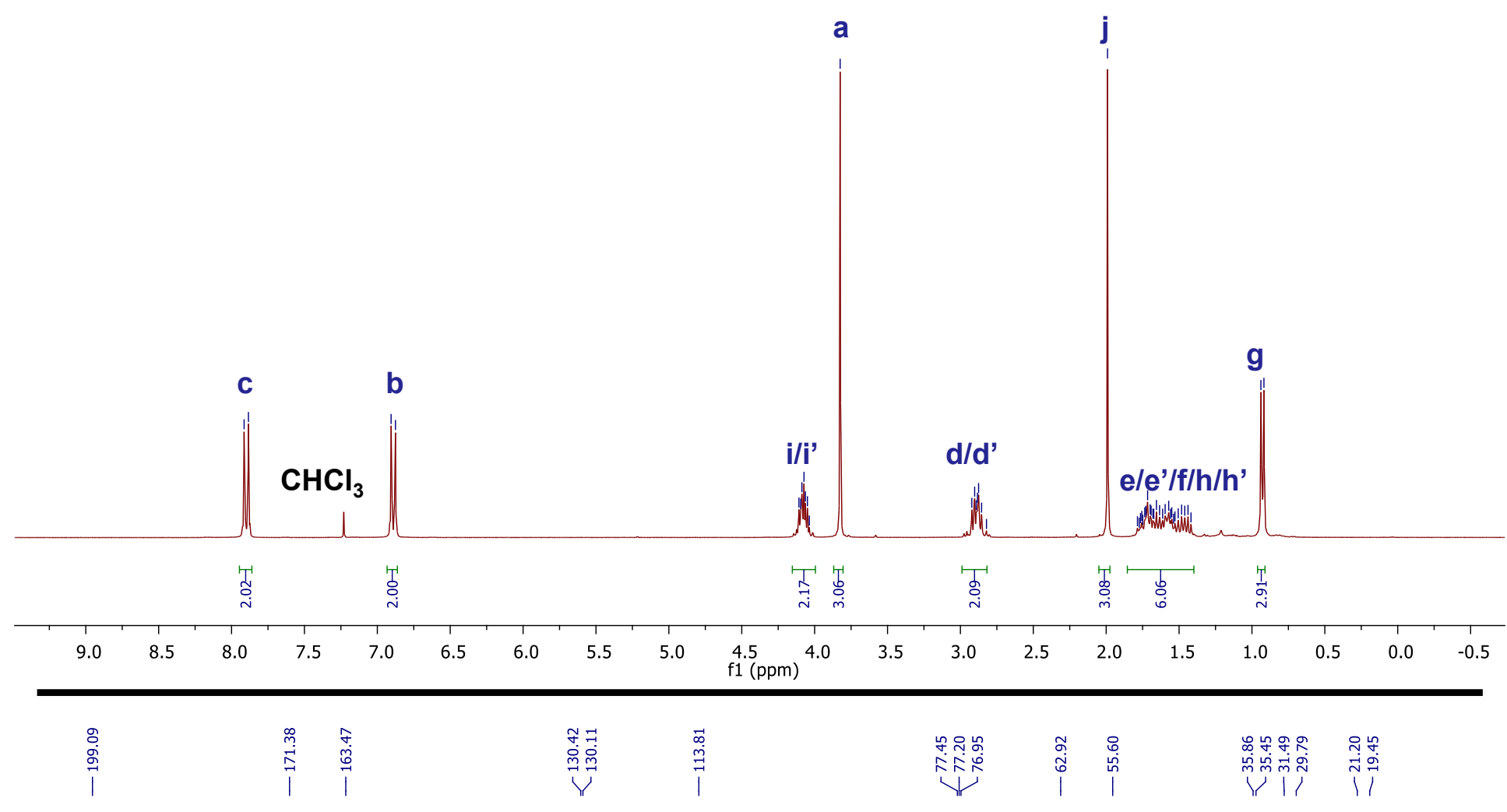

${ }^{13}$ C-NMR (126 MHz; $\mathrm{CDCl}_{3}$ )
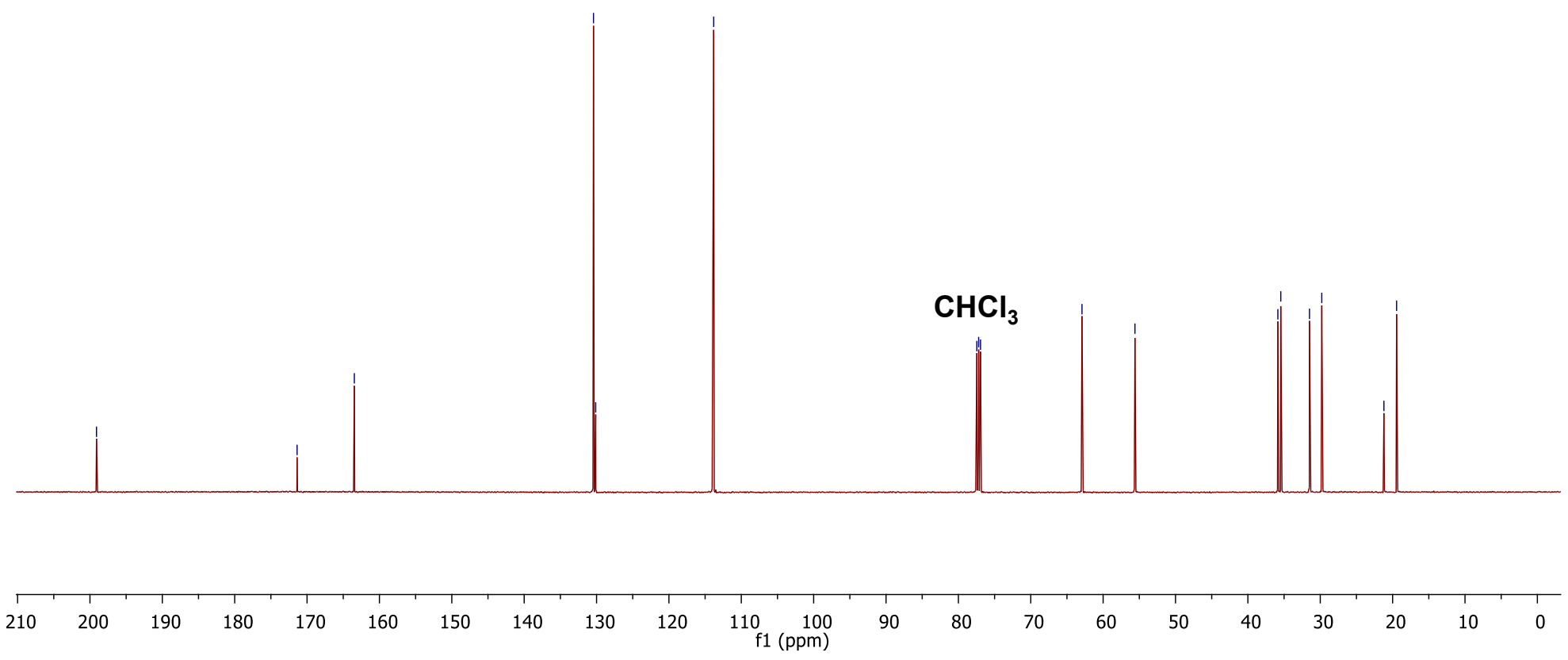
${ }^{1} \mathrm{H}-\mathrm{NMR}\left(300 \mathrm{MHz} ; \mathrm{CDCl}_{3}\right.$ )

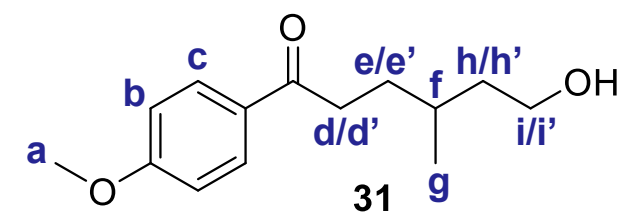

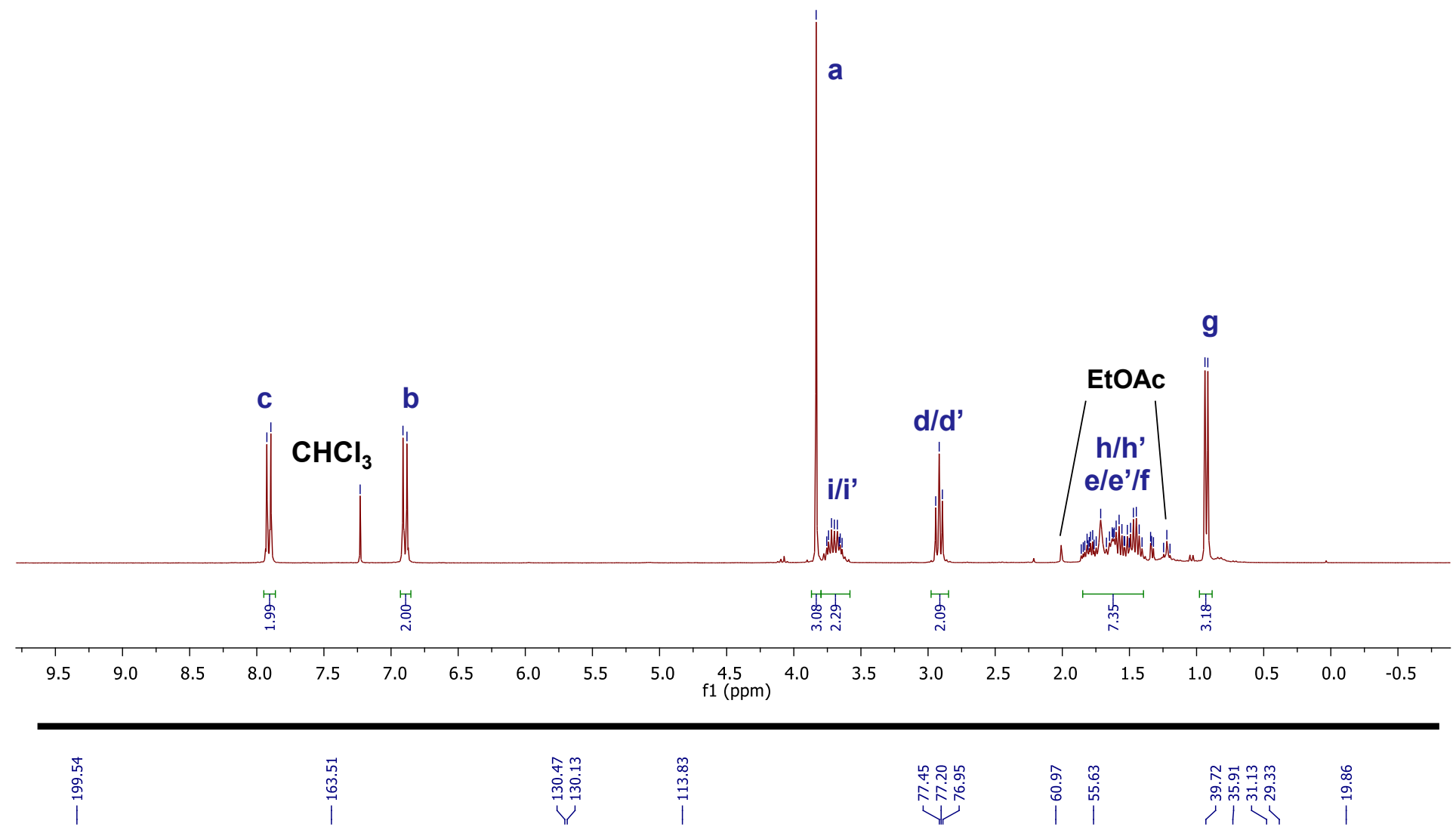

${ }^{13} \mathrm{C}-\mathrm{NMR}$ (126 MHz; $\mathrm{CDCl}_{3}$ )

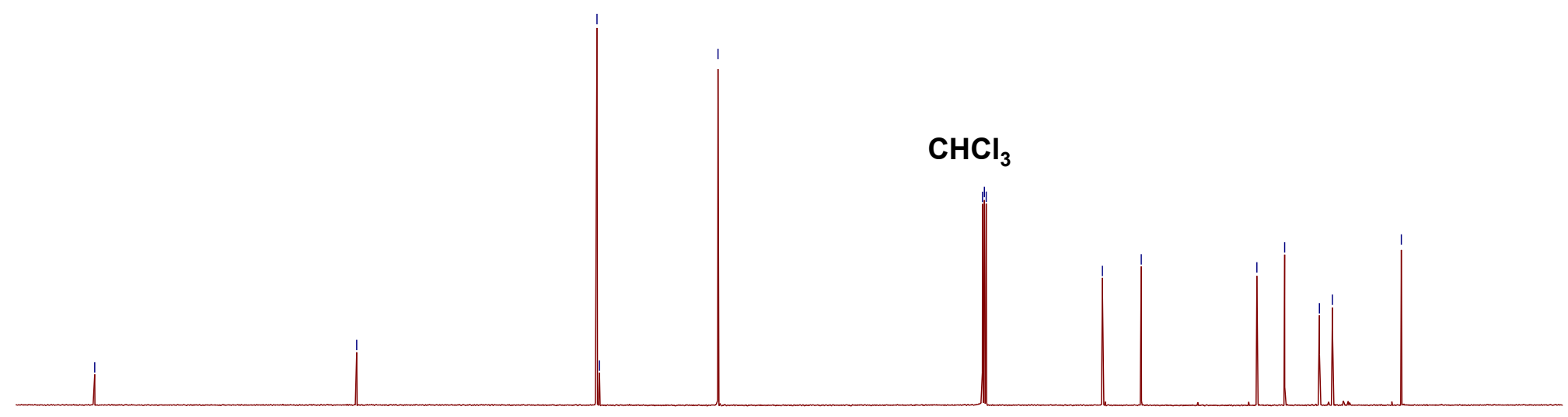

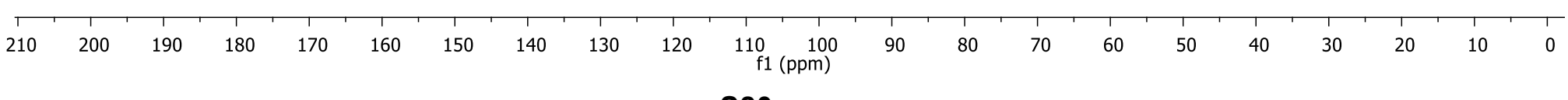


${ }^{1} \mathrm{H}-\mathrm{NMR}\left(300 \mathrm{MHz} ; \mathrm{CDCl}_{3}\right.$ )
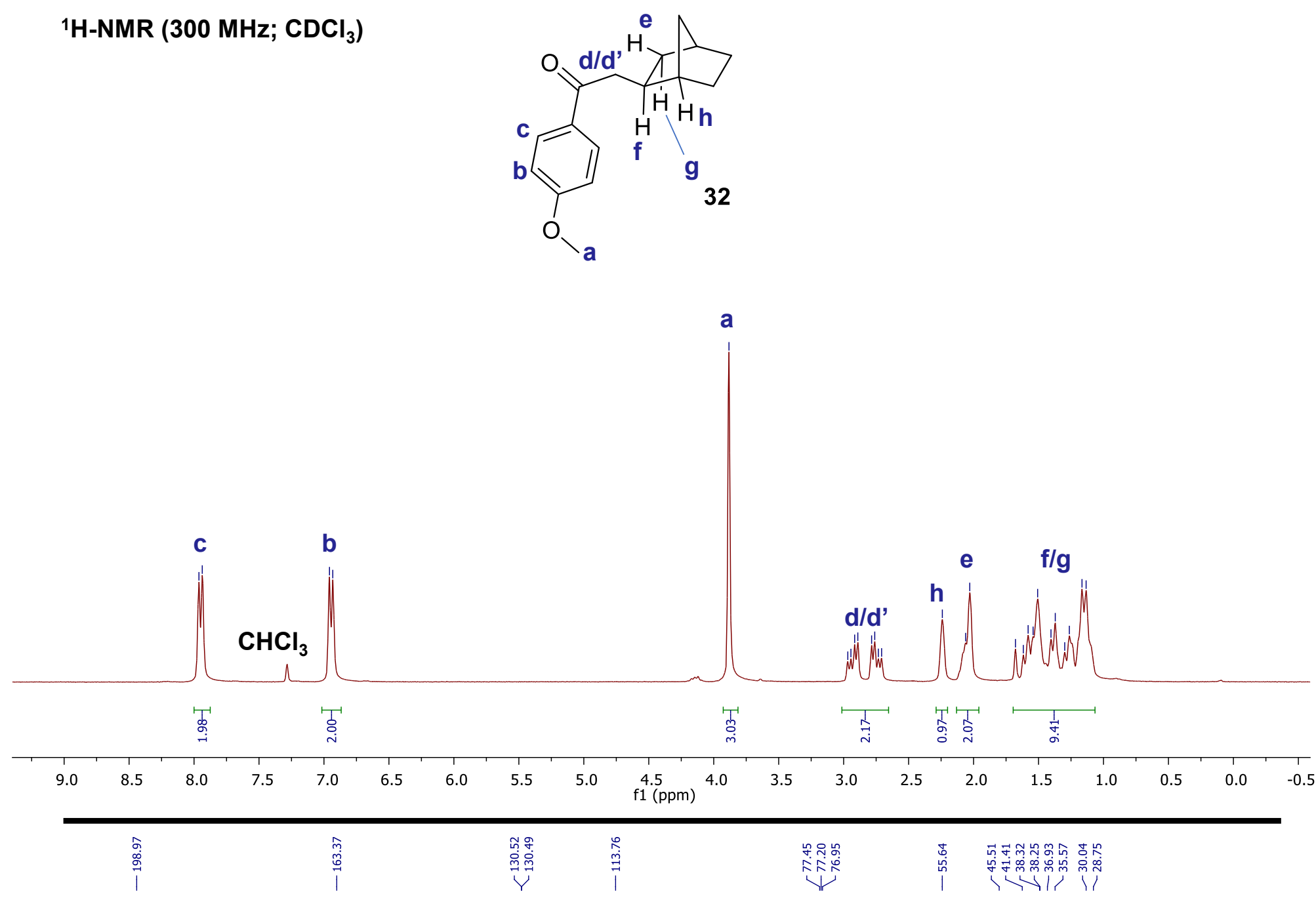

${ }^{13}$ C-NMR (126 MHz; $\mathrm{CDCl}_{3}$ )

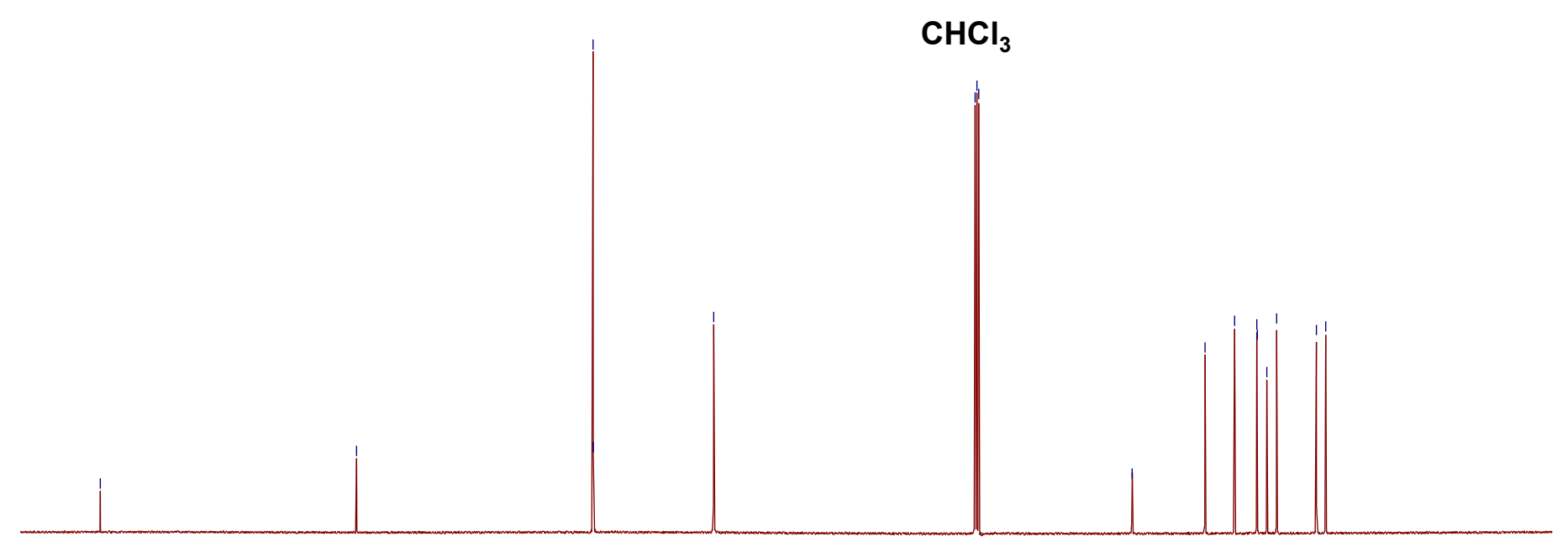

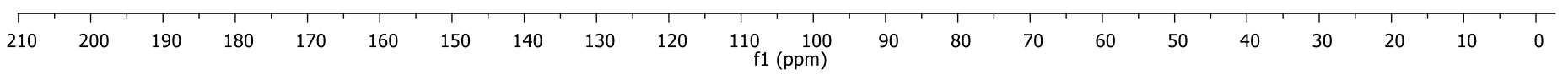



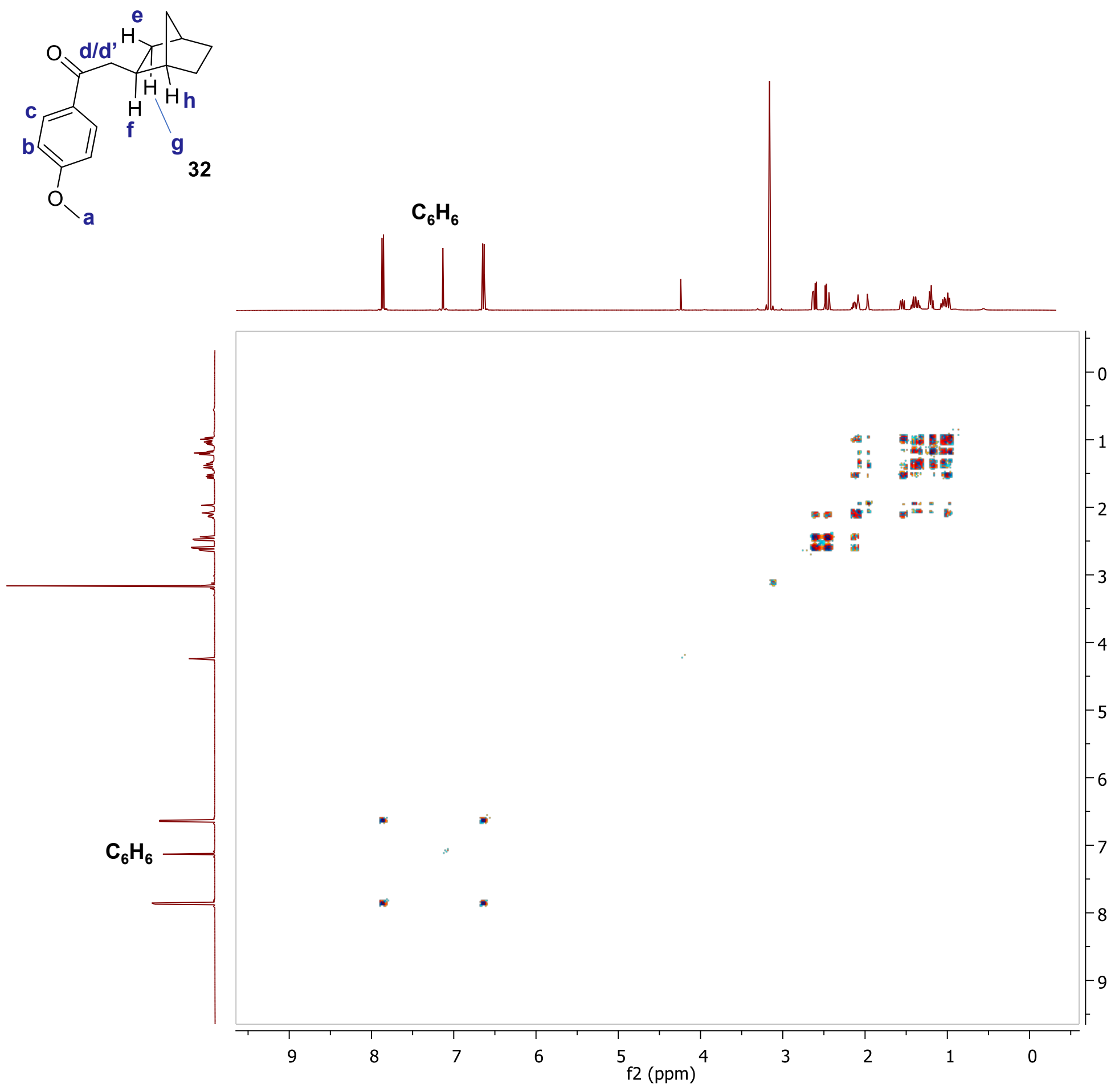

The stereochemistry of 32 could not be resolved by NMR. Radical additions typically yield the exo-addition products, see: a) Curran, D. P.; Porter, N. A.; Giese, B. Stereochemistry of Radical Reactions; VCH: New York, 1996; b) Davies, D. I.; Parfitt, L. T.; Alden, C. K.; Claisse, J. A. J. Chem. Soc. C 1969, 1585-1590; c) Fawcett, F. S. Chem. Rev. 1950, 47, 219-274. 


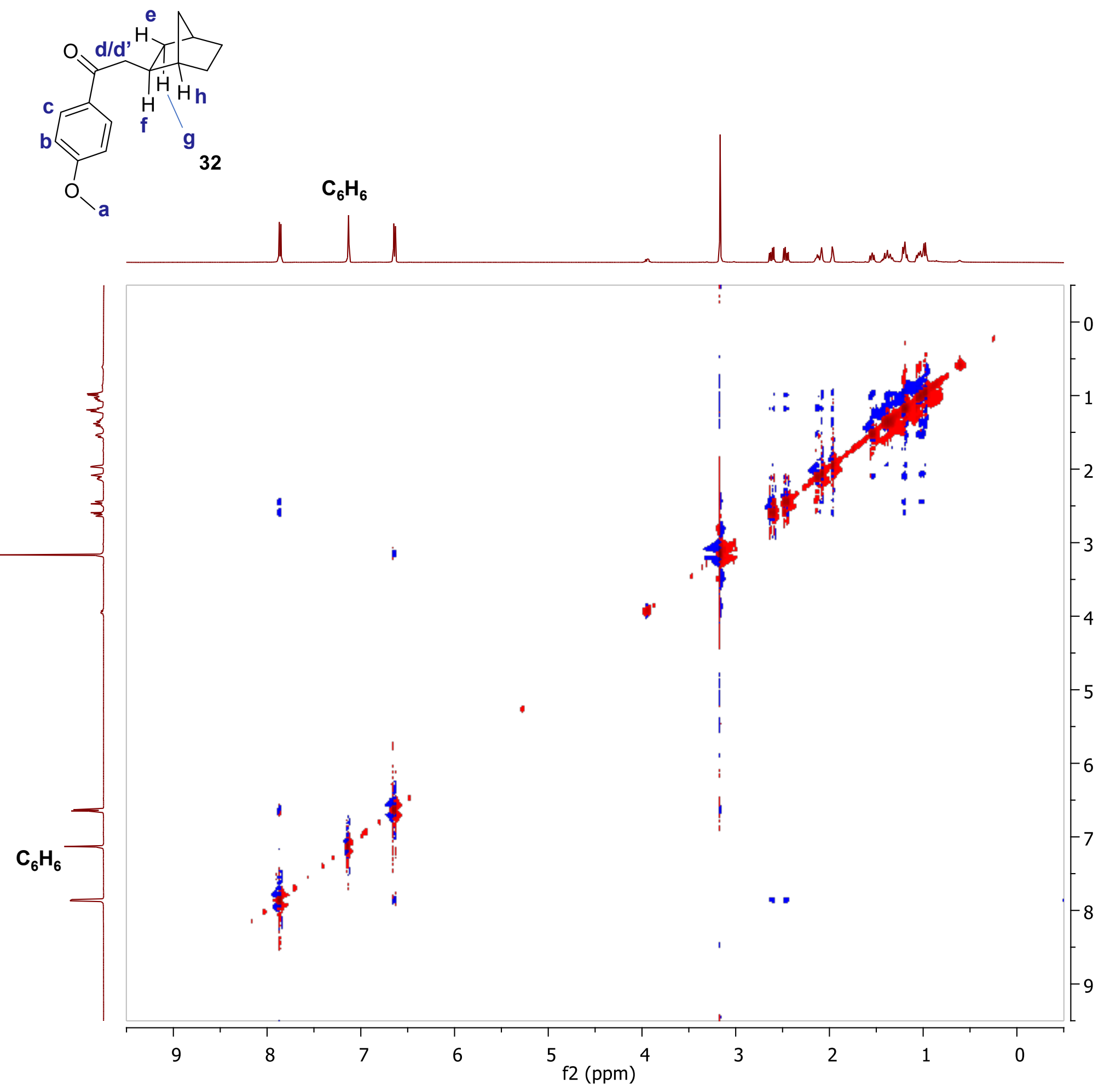


${ }^{1} \mathrm{H}-\mathrm{NMR}\left(300 \mathrm{MHz}^{\mathrm{CDCl}}{ }_{3}\right)$

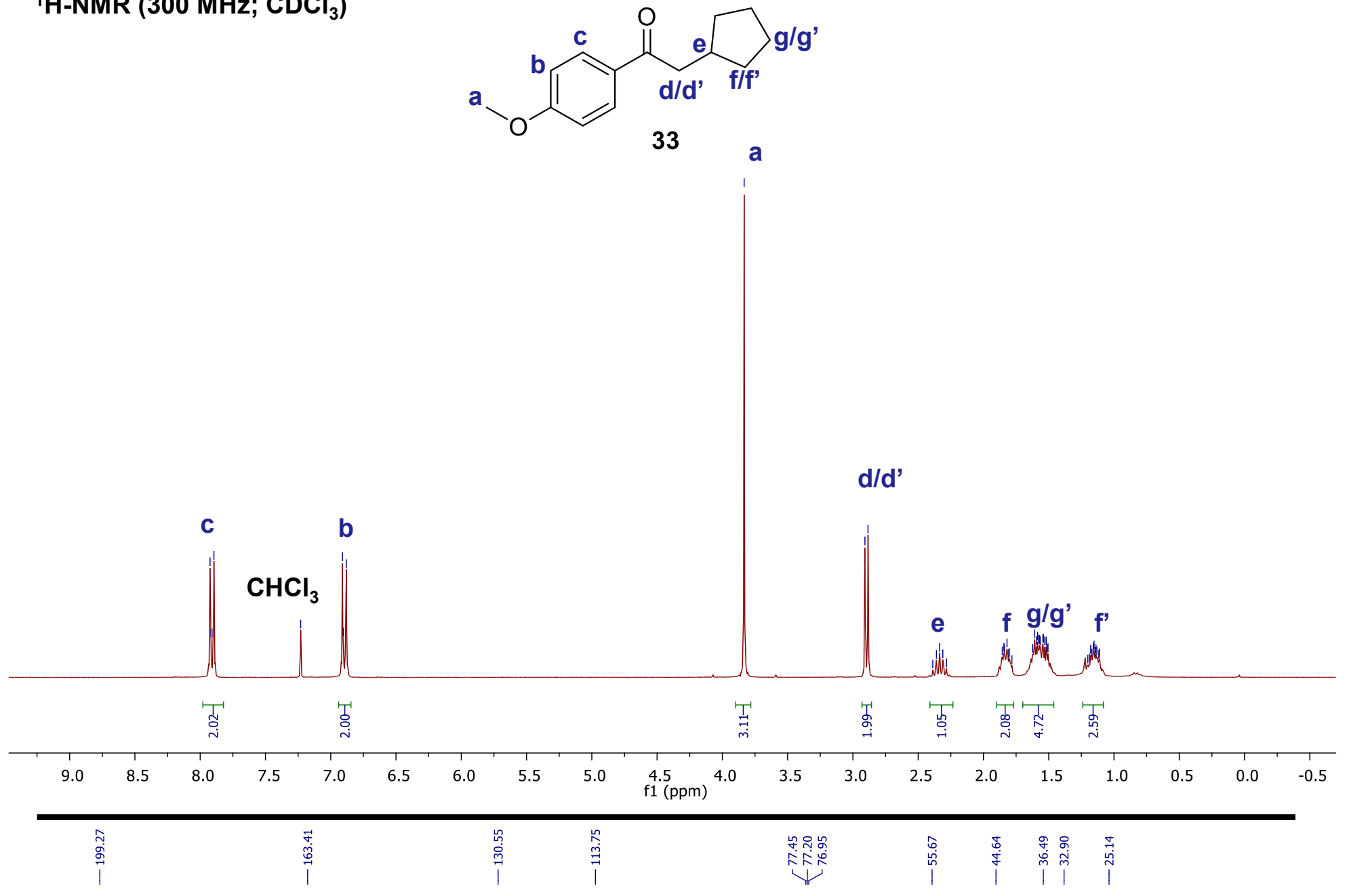

${ }^{13} \mathrm{C}-\mathrm{NMR}\left(126 \mathrm{MHz} ; \mathrm{CDCl}_{3}\right)$

\section{$\mathrm{CHCl}_{3}$}

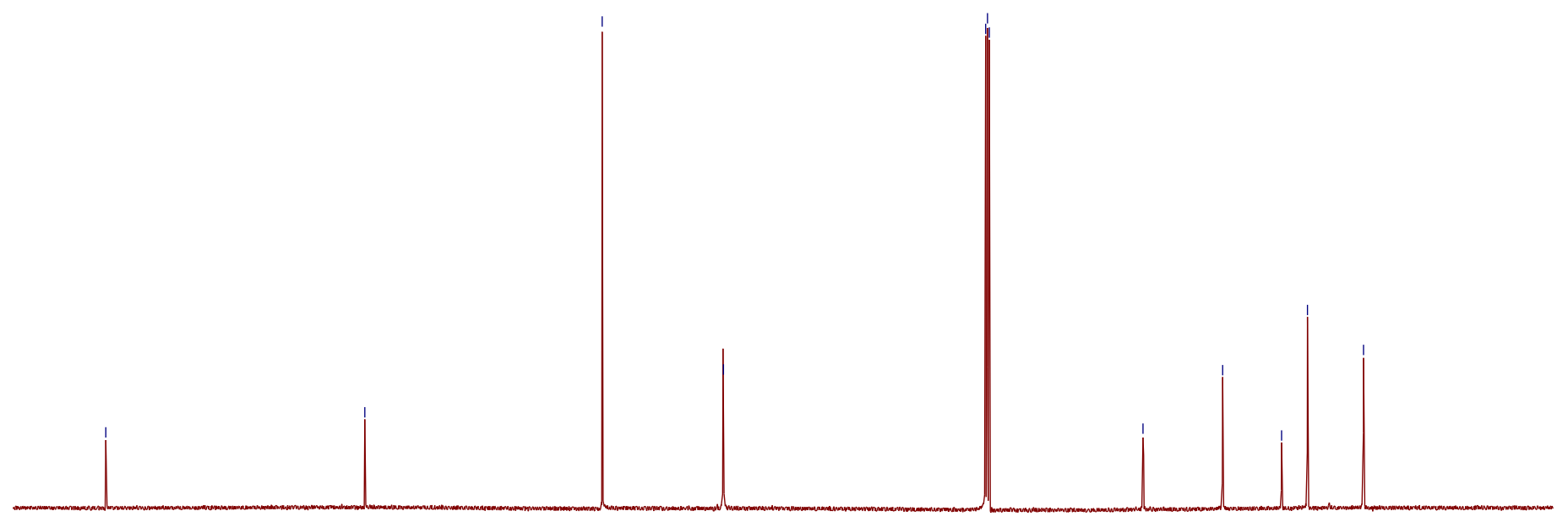

$\begin{array}{lllllllllll}210 & 200 & 190 & 180 & 170 & 160 & 150 & 140 & 130 & 120 & 110 \begin{array}{l}100 \\ \mathrm{f} 1(\mathrm{ppm})\end{array}\end{array}$


${ }^{1} \mathrm{H}-\mathrm{NMR}\left(300 \mathrm{MHz} ; \mathrm{CDCl}_{3}\right.$ )<smiles>COc1ccc(C(=O)C(Cl)C2CCC([Al])CC2)cc1</smiles>
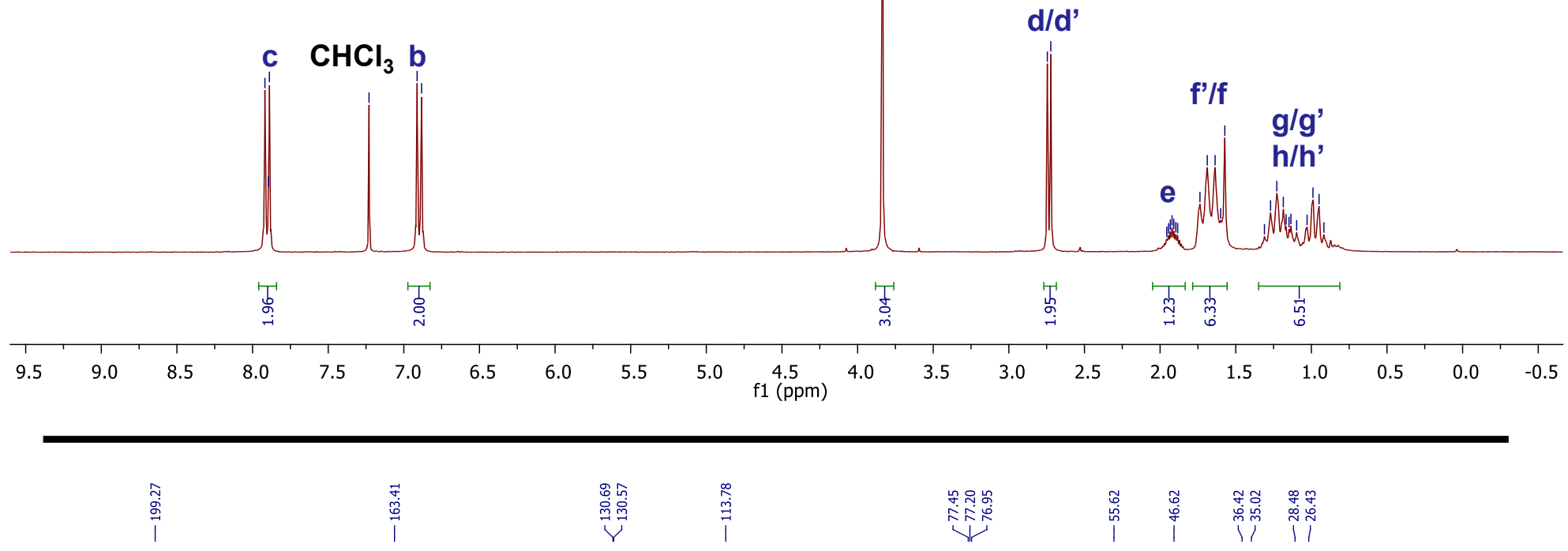

${ }^{13} \mathrm{C}-\mathrm{NMR}$ (126 MHz; $\mathrm{CDCl}_{3}$ )
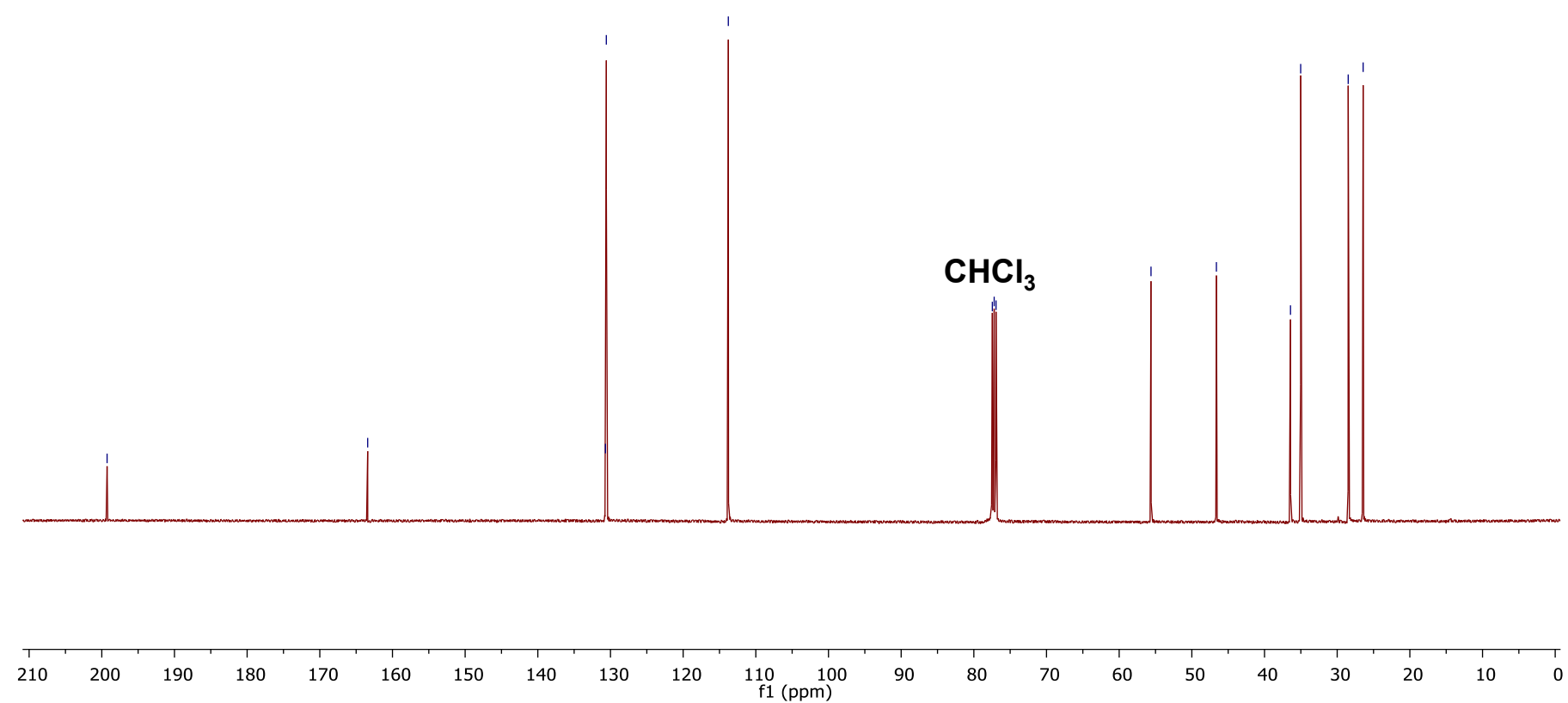
${ }^{1} \mathrm{H}-\mathrm{NMR}$ (300 MHz; $\mathrm{CDCl}_{3}$ )

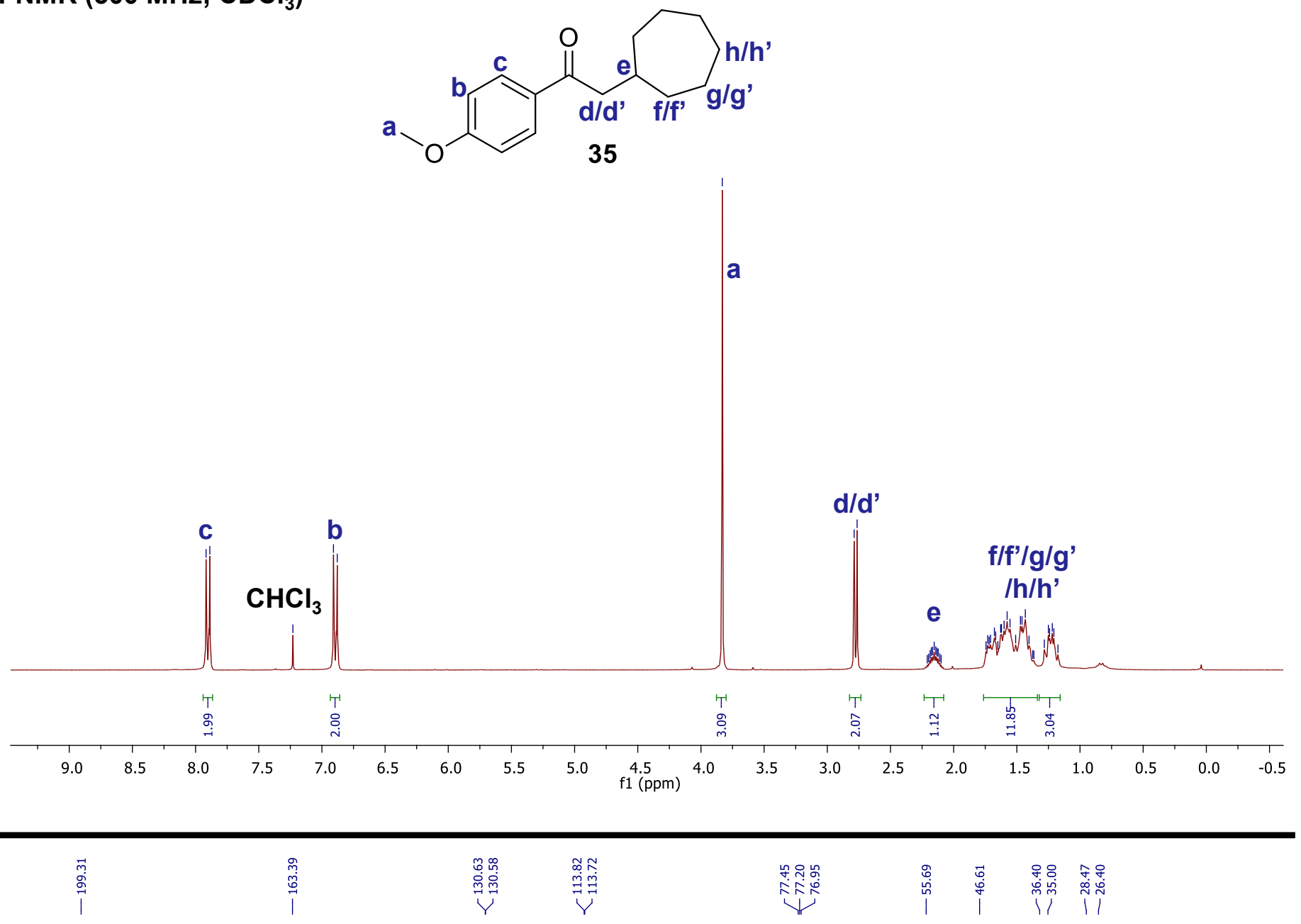

${ }^{13} \mathrm{C}-\mathrm{NMR}$ (126 MHz; $\mathrm{CDCl}_{3}$ )
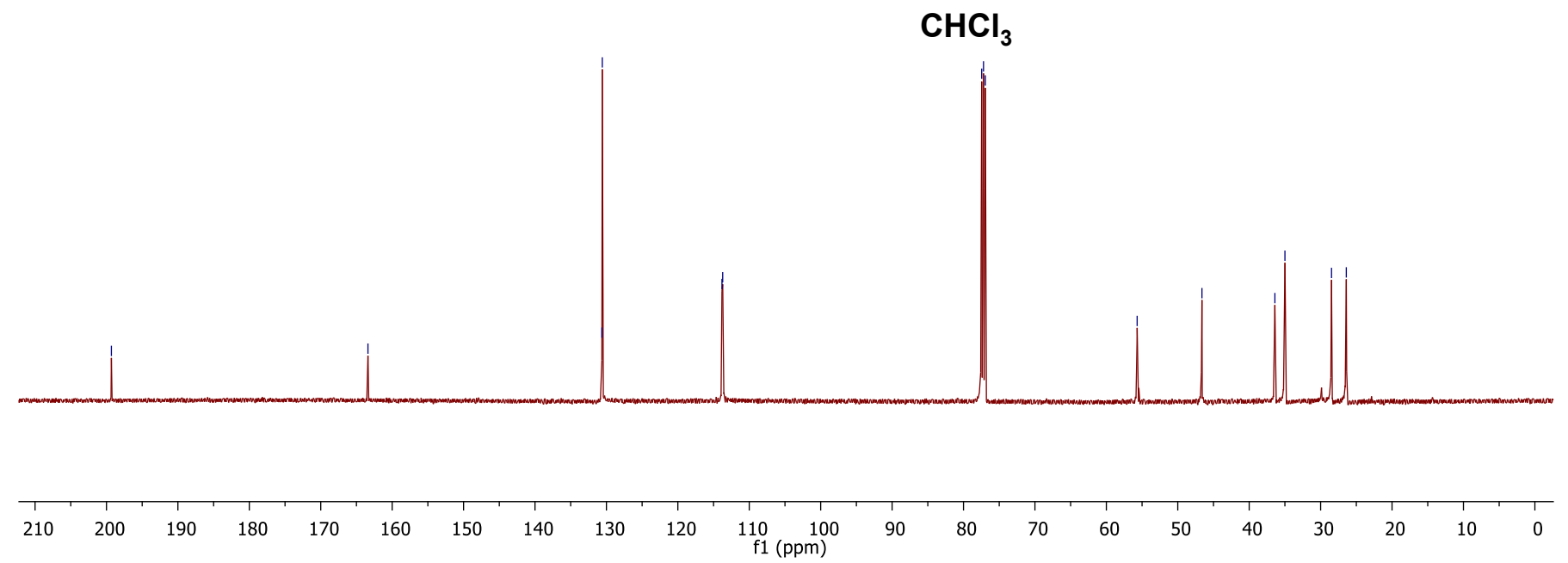
${ }^{1} \mathrm{H}-\mathrm{NMR}$ (300 MHz; $\mathrm{CDCl}_{3}$ )

a<smiles>O=C(c1ccc(OCl)cc1)C(Cl)C1CCCC([AlH2])CC1[Mg]</smiles>
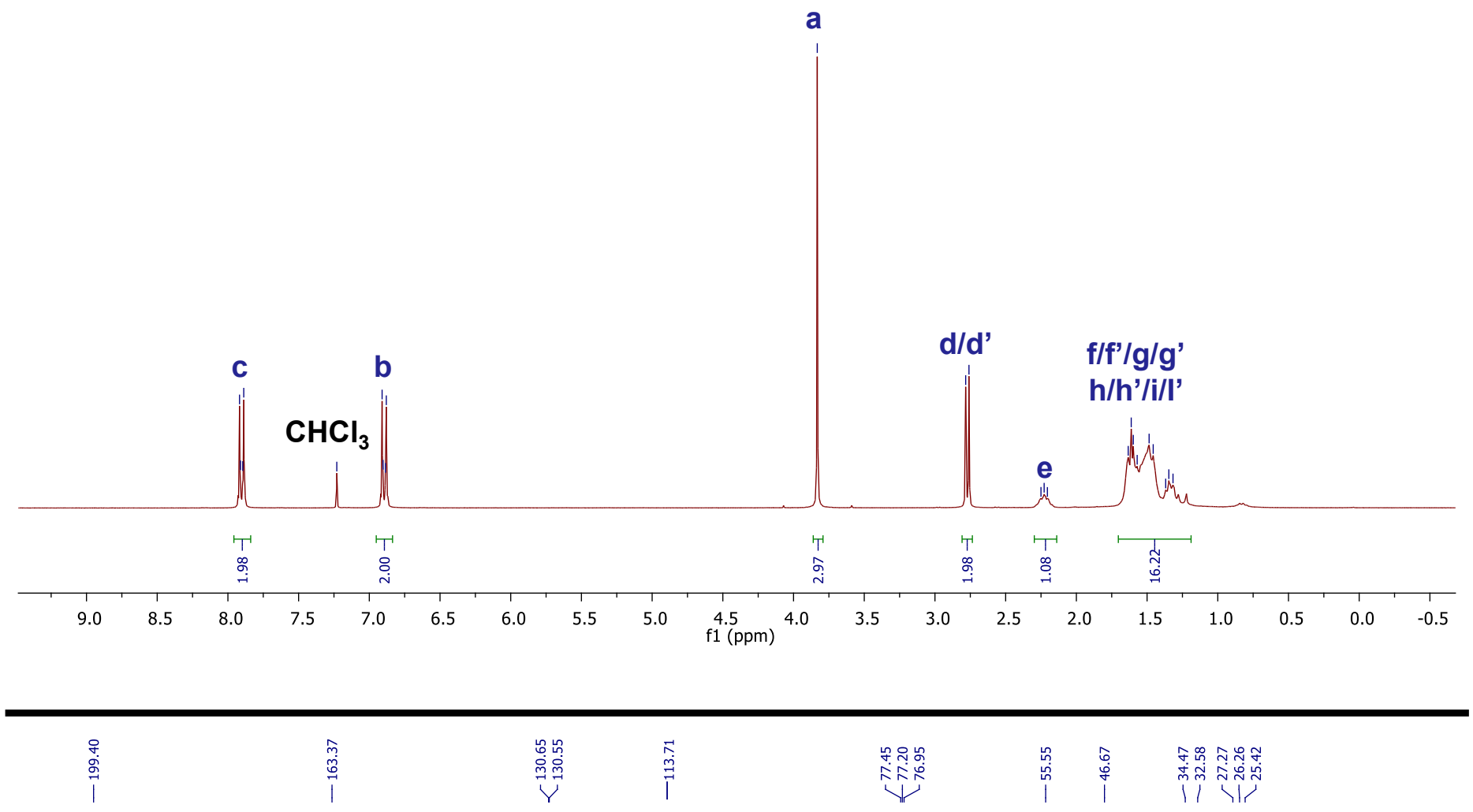

${ }^{13} \mathrm{C}-\mathrm{NMR}\left(126 \mathrm{MHz} ; \mathrm{CDCl}_{3}\right.$ )

\section{$\mathrm{CHCl}_{3}$}

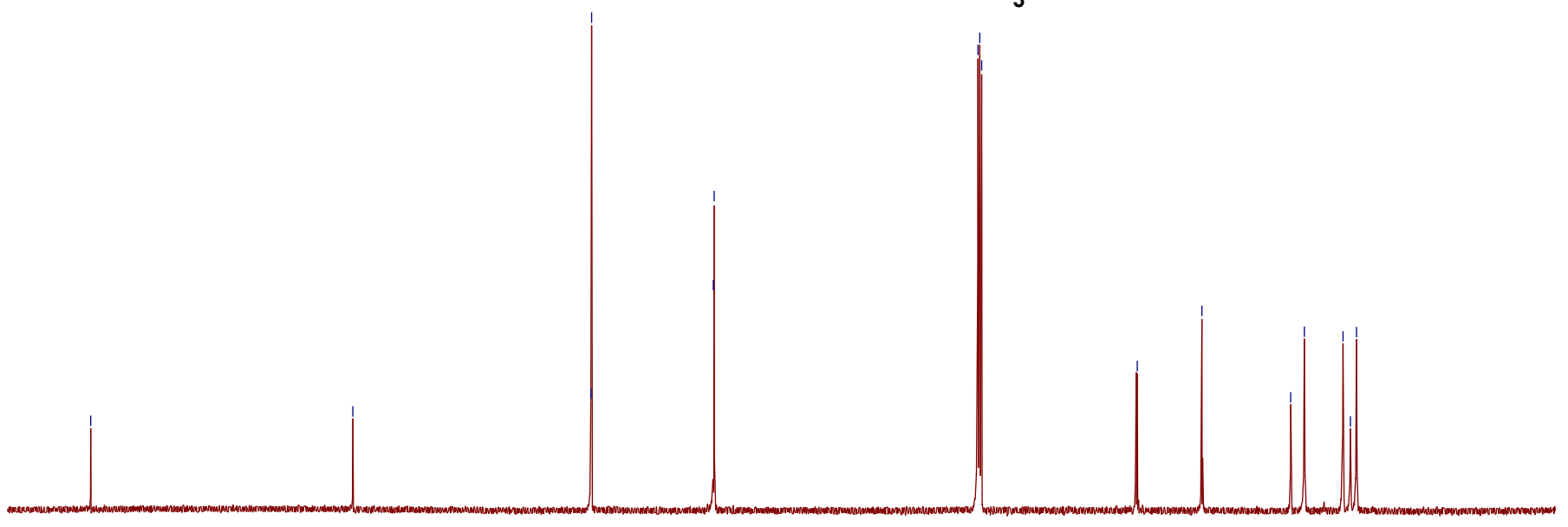


${ }^{1} \mathrm{H}-\mathrm{NMR}$ (300 MHz; $\mathrm{CDCl}_{3}$ )
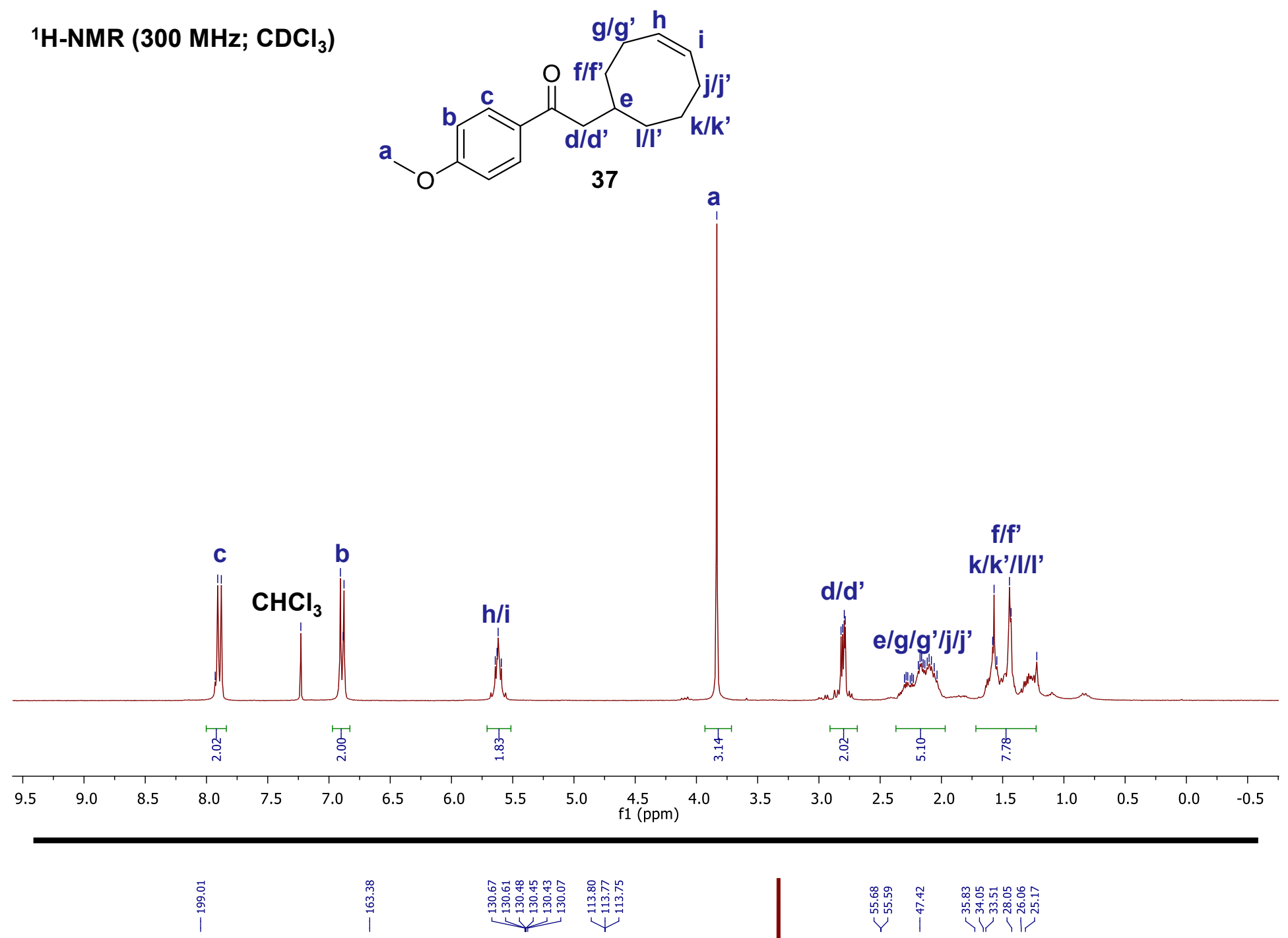

${ }^{13} \mathrm{C}-\mathrm{NMR}$ (126 MHz; $\mathrm{CDCl}_{3}$ )

$\mathrm{CHCl}_{3}$

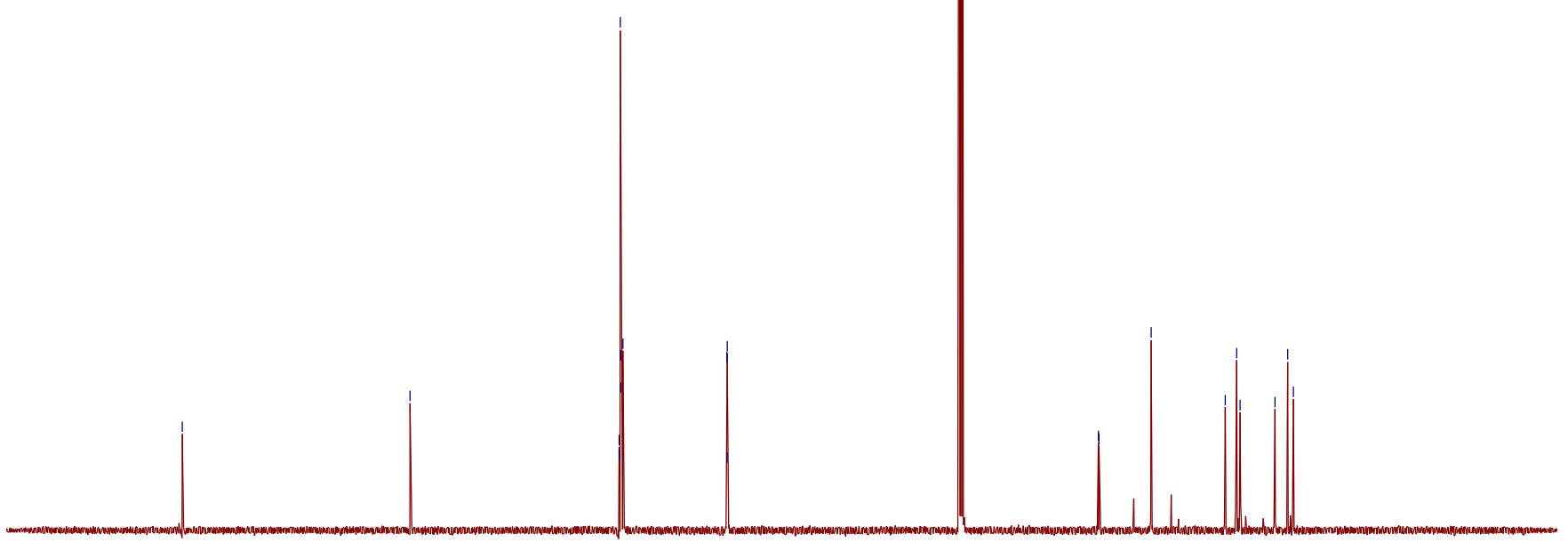

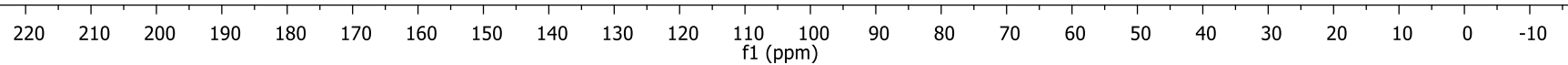




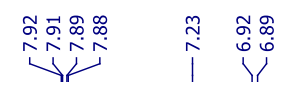

${ }^{1} \mathrm{H}-\mathrm{NMR}\left(300 \mathrm{MHz}^{\mathrm{CDCl}}{ }_{3}\right)$

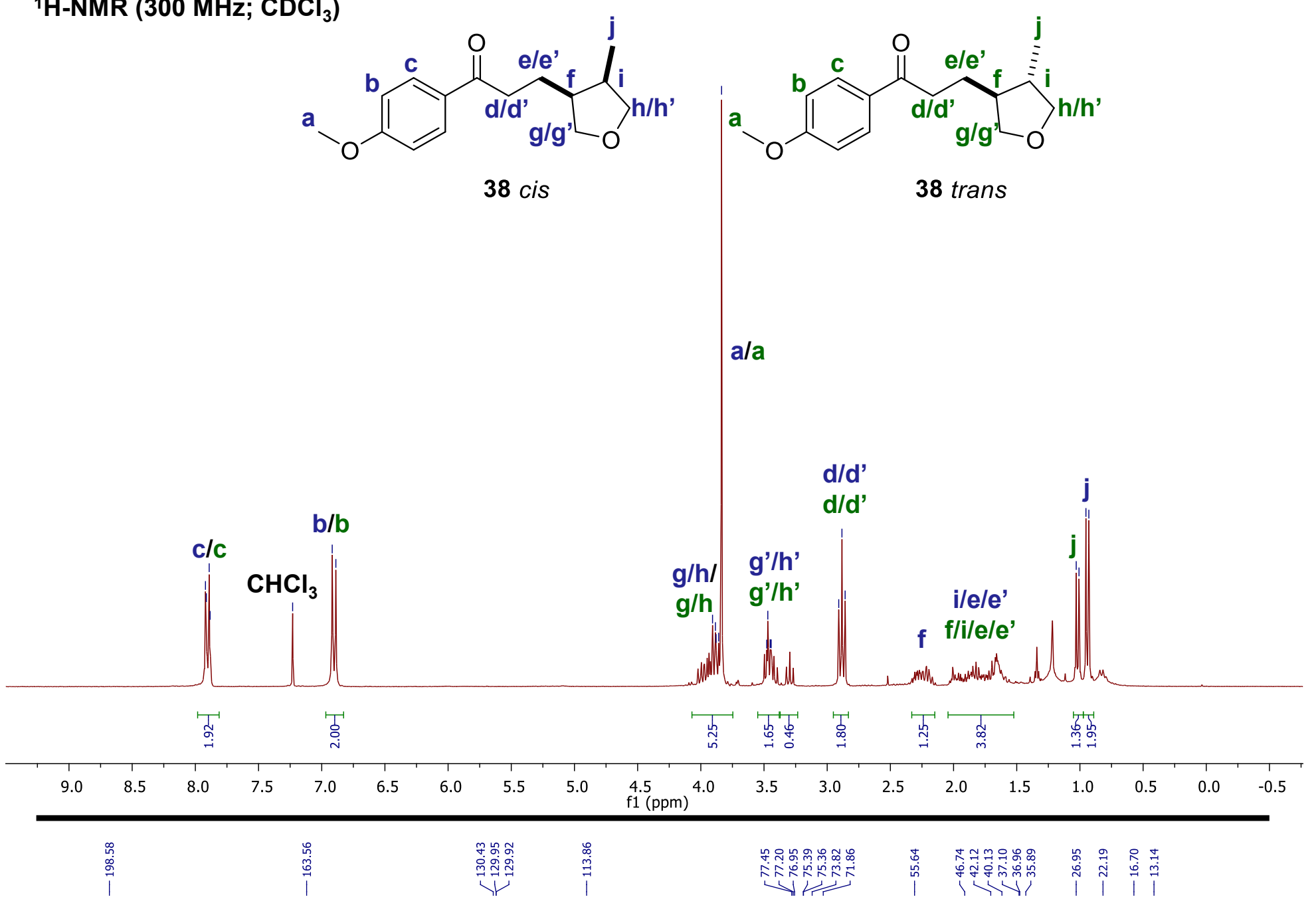

${ }^{13} \mathrm{C}-\mathrm{NMR}\left(126 \mathrm{MHz}^{\mathrm{CDCl}}{ }_{3}\right)$

The major diastereomers for 38 and 39 were determined to be cis by the gammagauche interaction observed in the 13C NMR, see: Silverstein, R. M.; Kiemle, D. J.; Webster, F. X.; Bryce, D. L. Spectrometric identification of organic compounds; John Wiley \& Sons: New York, 2015.

For examples, the methyl group "j" in 38 -cis at 13.14 ppm is deshielded by 3.56 ppm relative to 38-trans. This has been previously observed in similar systems, see: a) Bentley, J.; Nilsson, P. A.; Parsons, A. F. J. Chem. Soc., Perkin Trans. 1 2002, 1461-1469; b) Bryans, J. S.; Large, J. M.; Parsons, A. F. J. Chem. Soc., Perkin Trans. 1 1999, 2897-2904; c) Riggi, I. D.; Gastaldi, S.; Surzur, J. M.; Betrand, M. P.; Virgili A. J. J. Org. Chem. 1992, 57, 6118-6125.

\section{$\mathrm{CHCl}_{3}$}

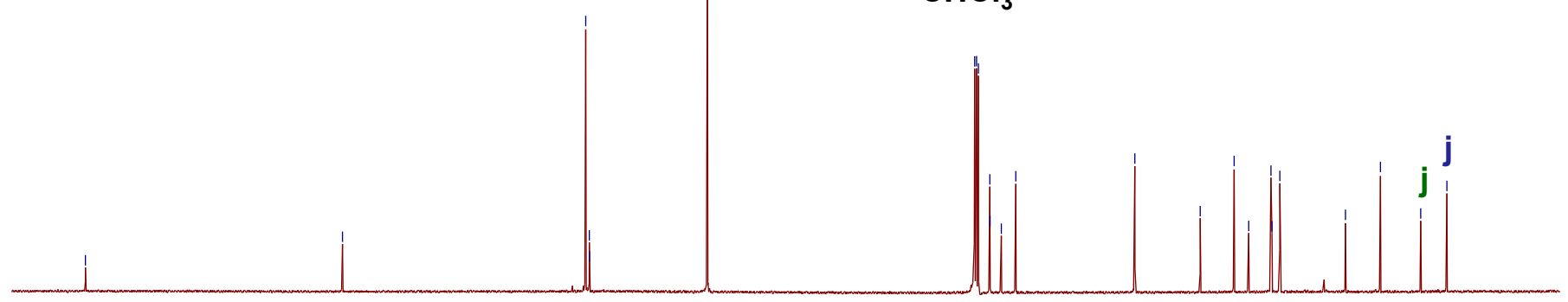

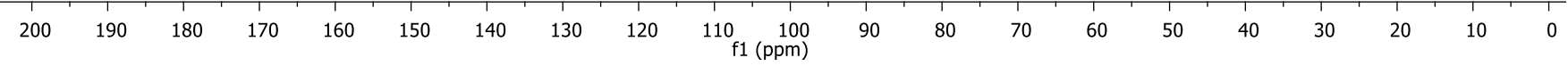


${ }^{1} \mathrm{H}-\mathrm{NMR}\left(300 \mathrm{MHz} ; \mathrm{CDCl}_{3}\right)$

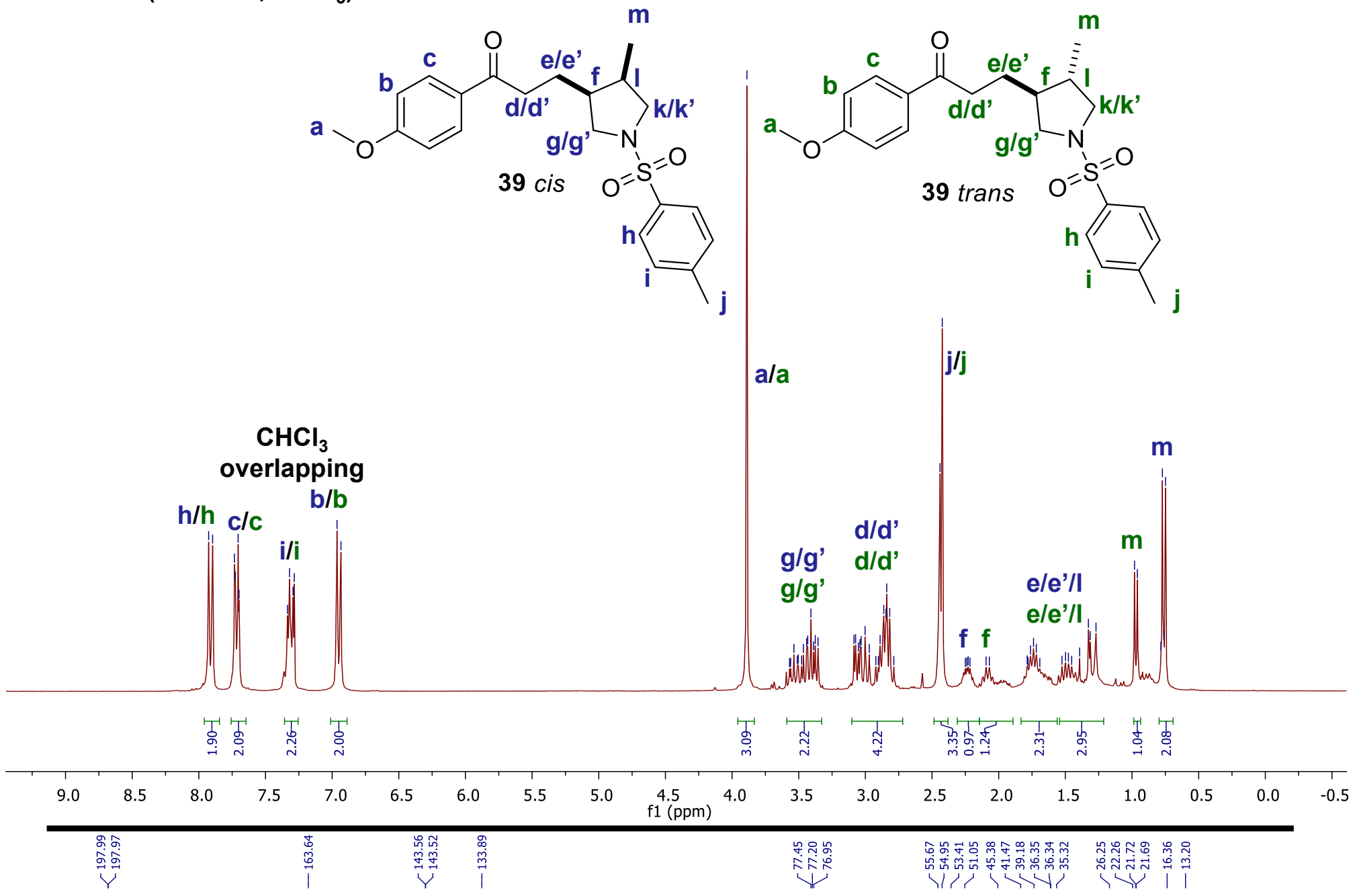

${ }^{13} \mathrm{C}-\mathrm{NMR}\left(126 \mathrm{MHz} ; \mathrm{CDCl}_{3}\right)$

The major diastereomer for 39 was determined to be cis by the gamma-gauche interaction observed in the 13C NMR, see: Silverstein, R. M.; Kiemle, D. J.; Webster, F. X.; Bryce, D. L. Spectrometric identification of organic compounds; John Wiley \& Sons: New York, 2015.

\section{$\mathrm{CHCl}_{3}$}

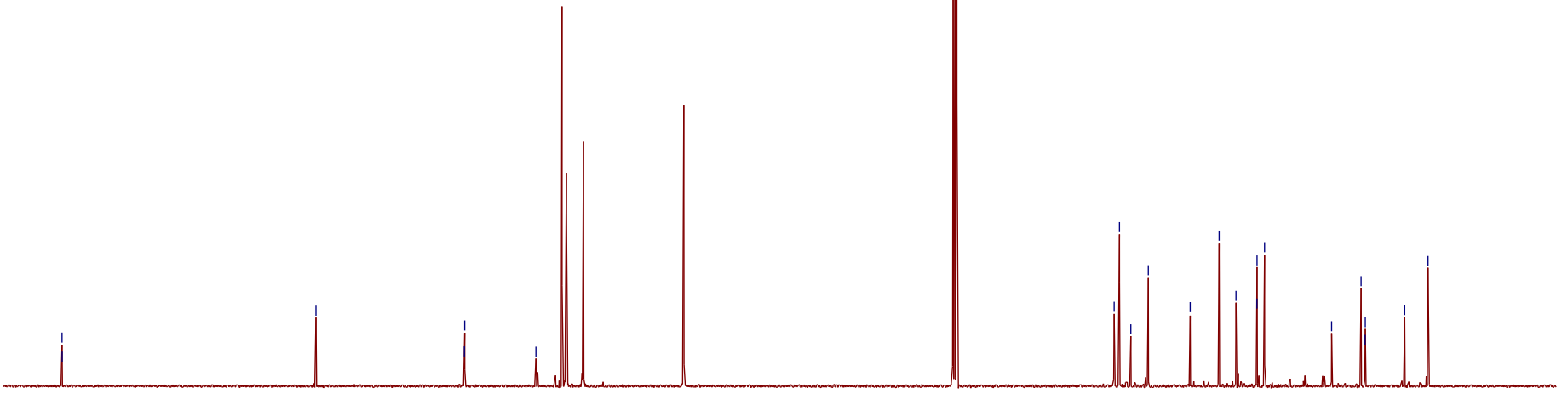

$\begin{array}{lllllllllll}200 & 190 & 180 & 170 & 160 & 150 & 140 & 130 & 120 & 110 & \begin{array}{l}100 \\ \mathrm{f} 1(\mathrm{ppm})\end{array}\end{array}$


${ }^{1} \mathrm{H}-\mathrm{NMR}\left(300 \mathrm{MHz} ; \mathrm{CDCl}_{3}\right.$ )

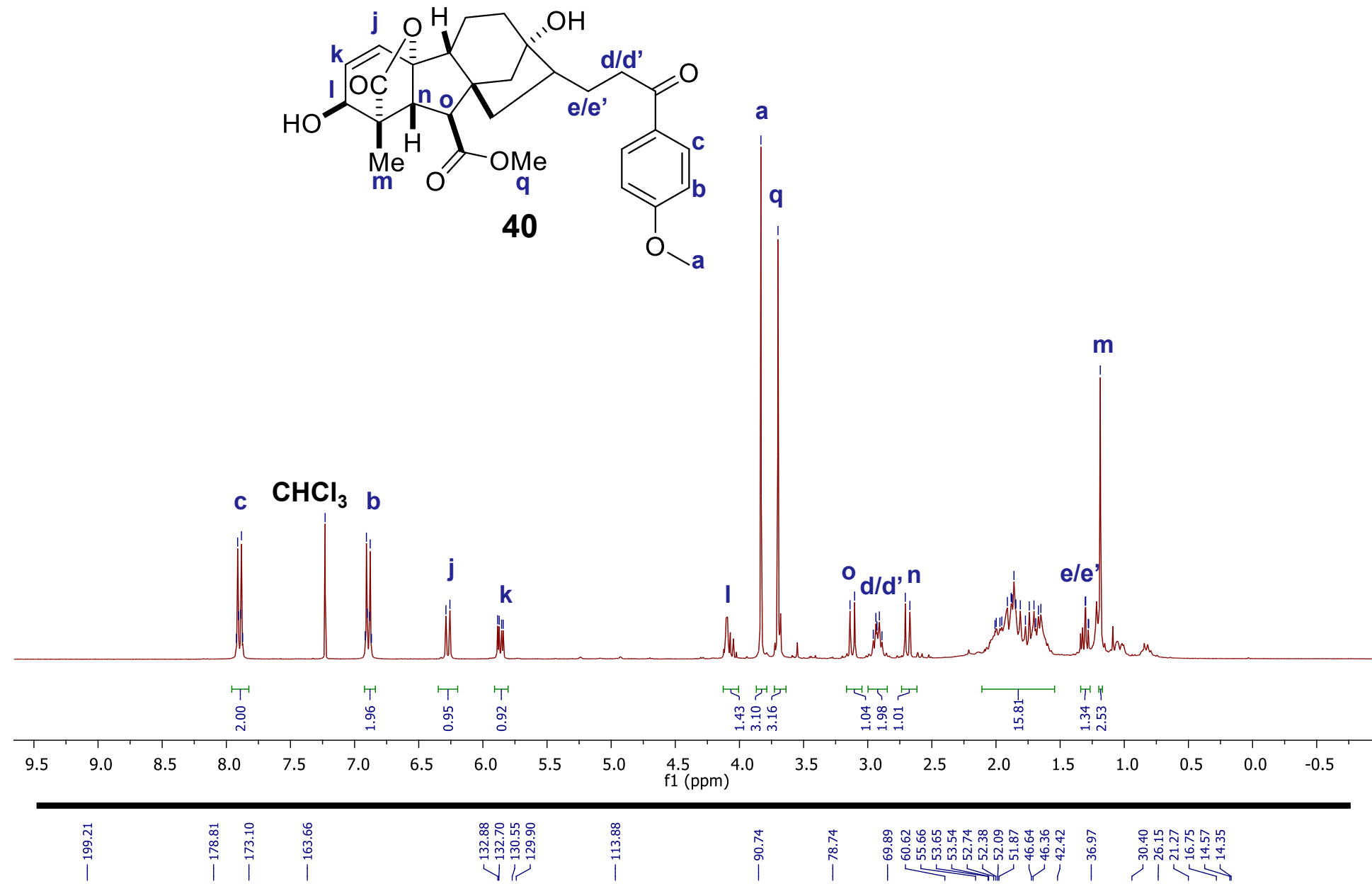

${ }^{13} \mathrm{C}-\mathrm{NMR}\left(126 \mathrm{MHz} ; \mathrm{CDCl}_{3}\right.$ )

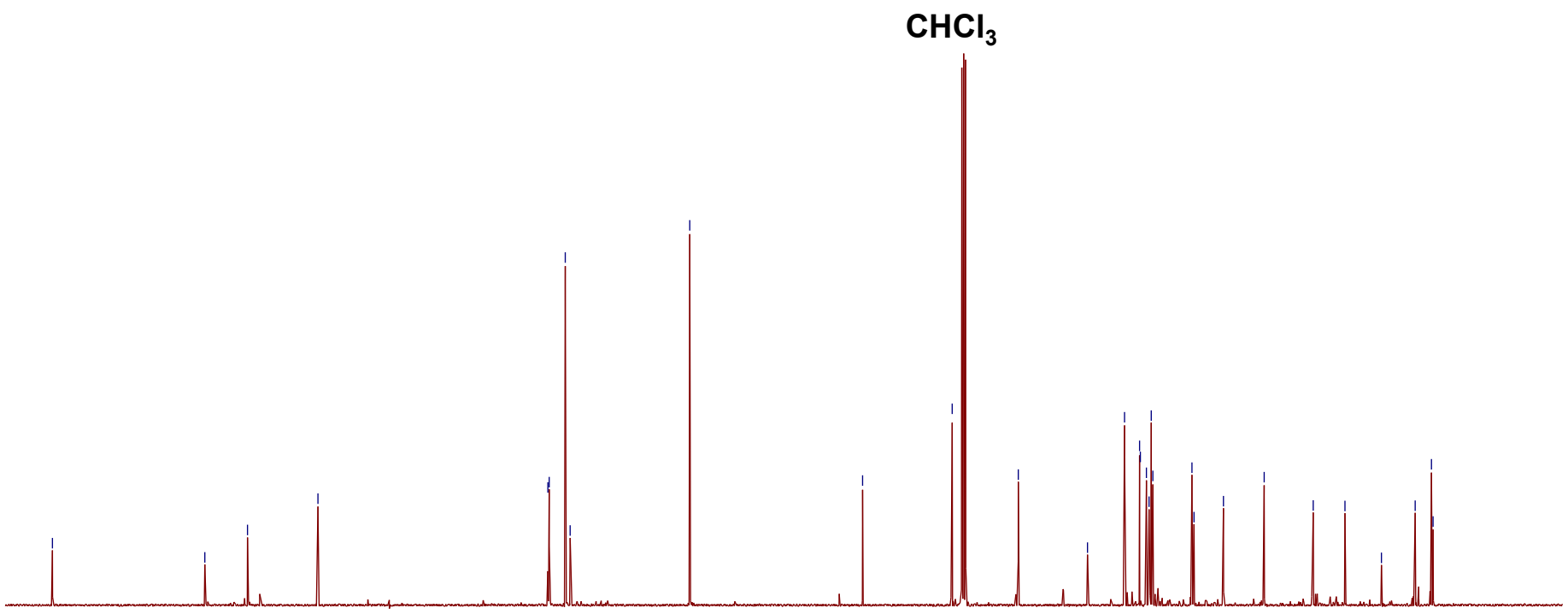

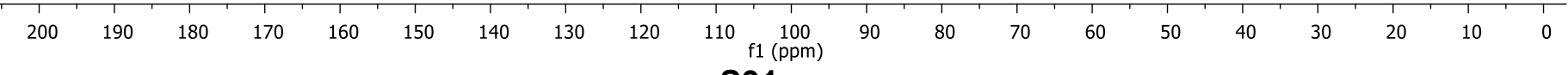


${ }^{1} \mathrm{H}-{ }^{1} \mathrm{H}$ NMR (500 MHz; $\left.\mathrm{C}_{6} \mathrm{D}_{6}\right)$ COSY

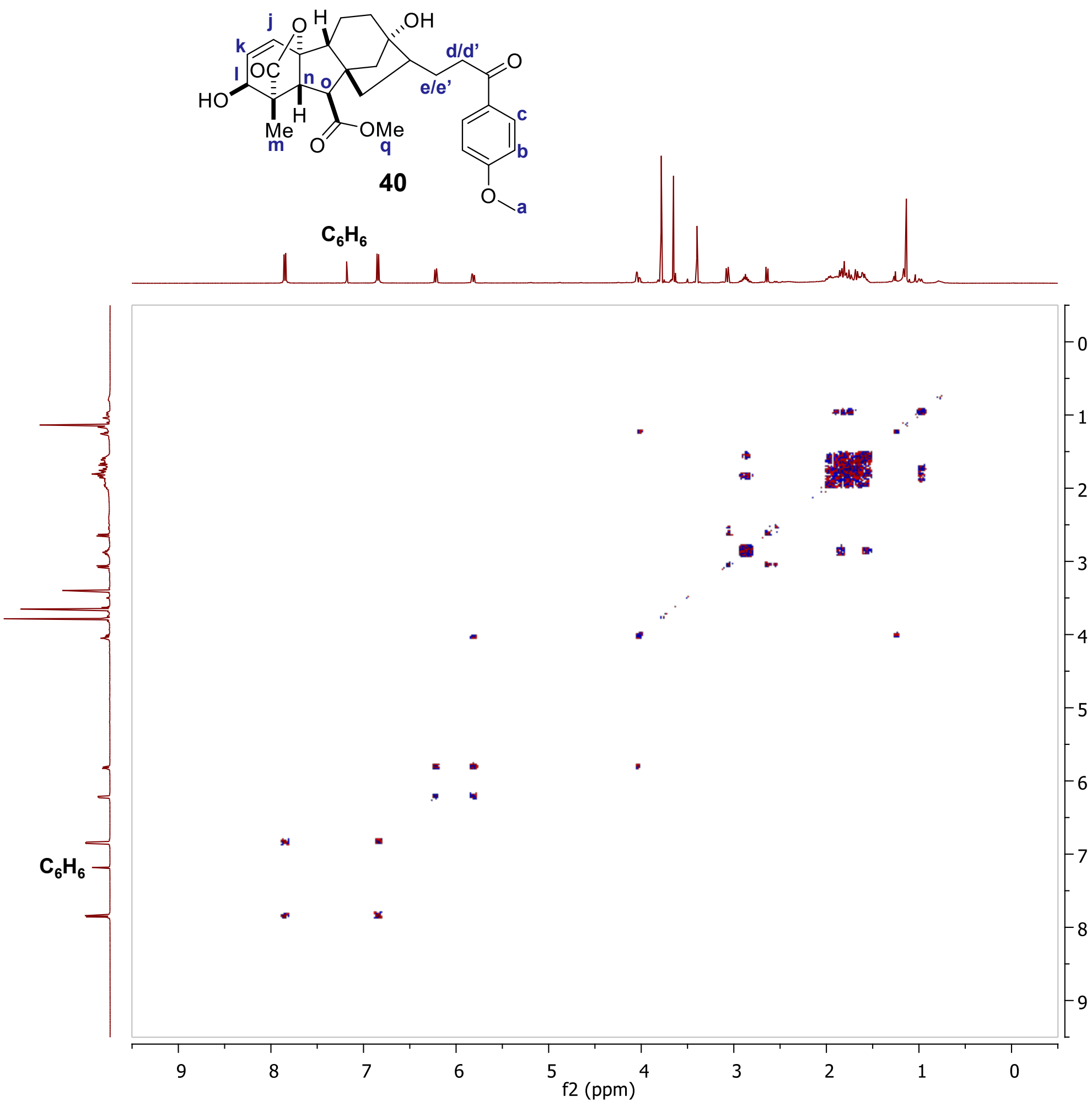




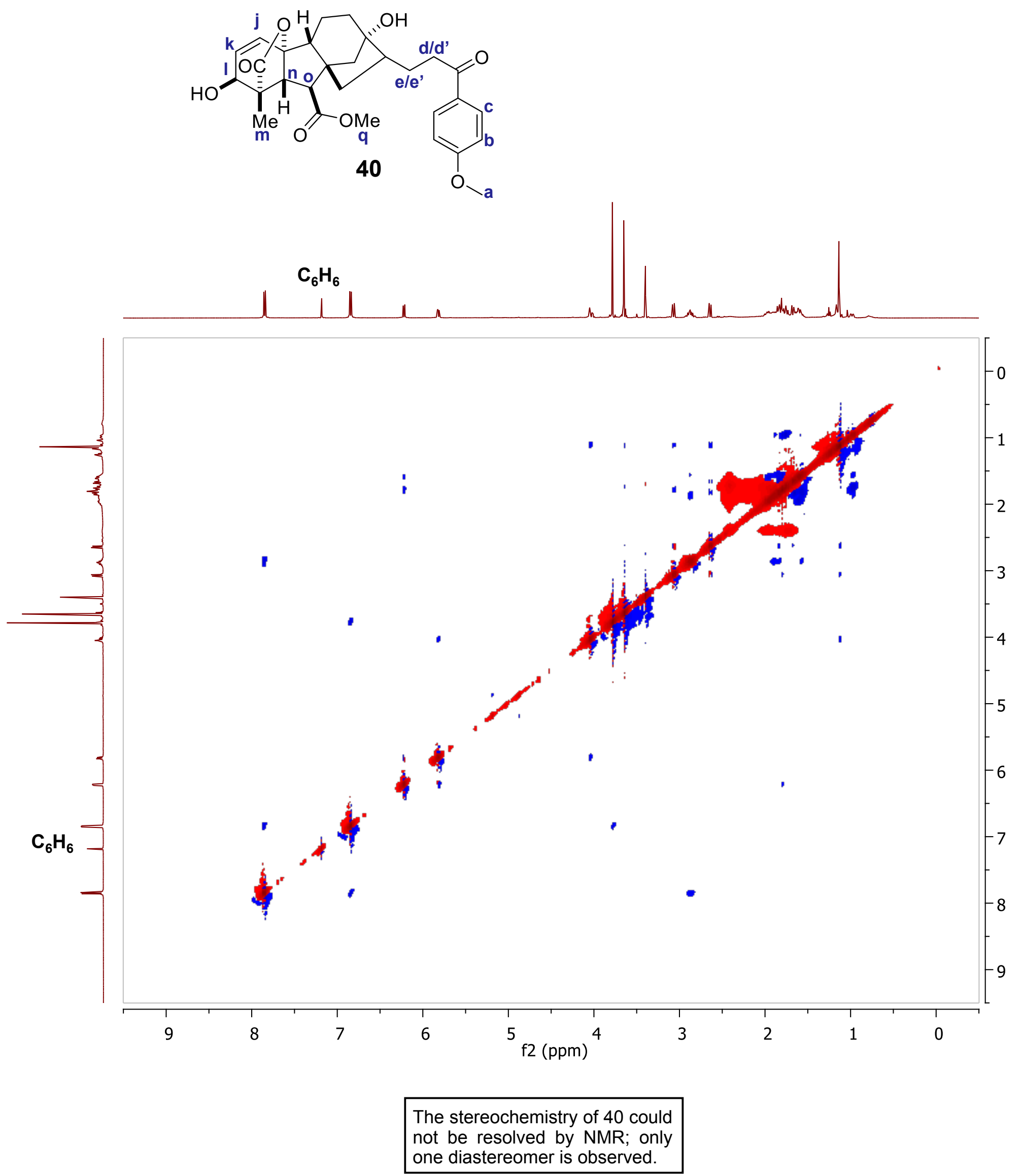



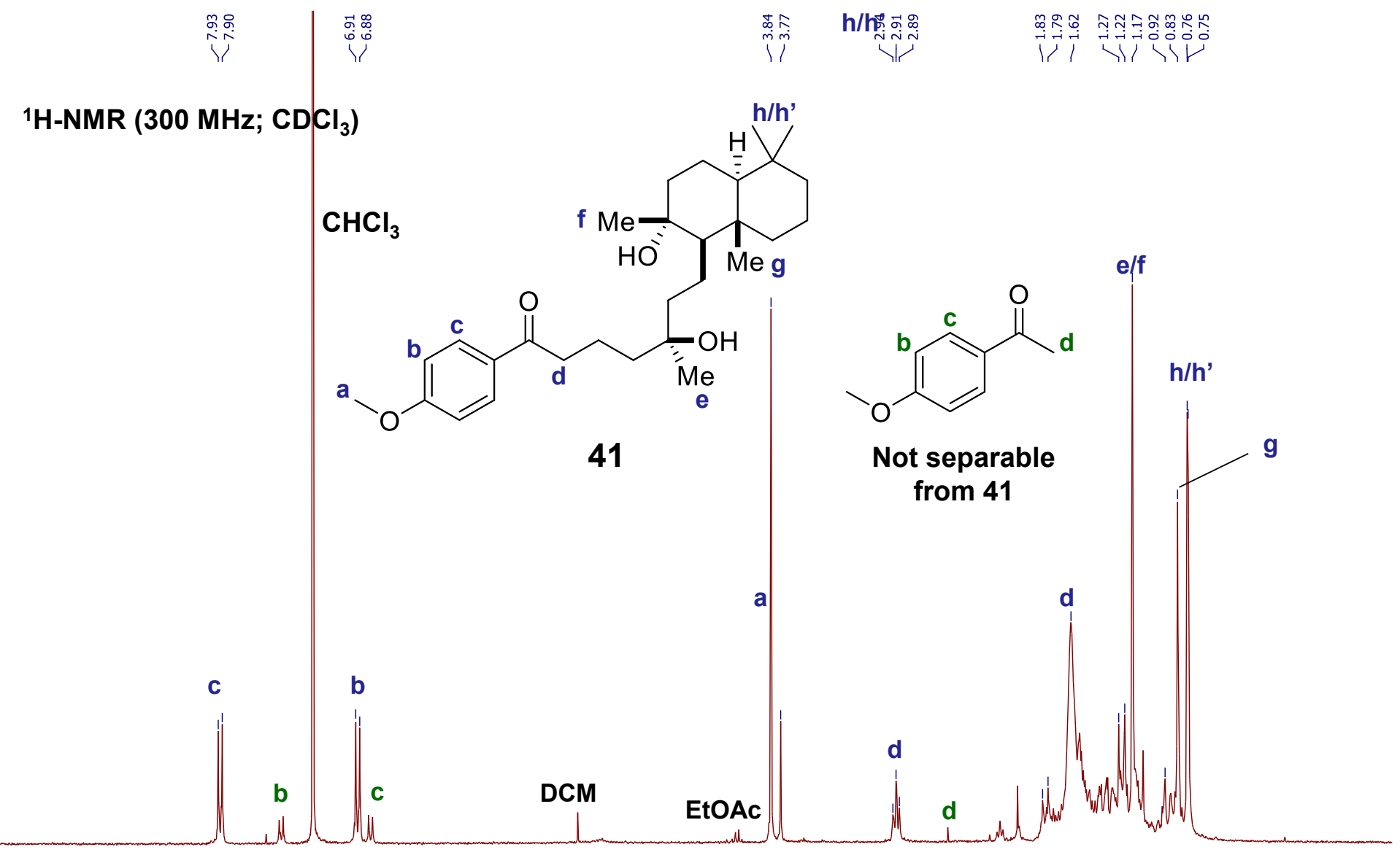

\begin{tabular}{|c|c|c|c|c|c|c|c|c|c|c|c|c|c|c|c|c|c|c|c|c|}
\hline & & & $\begin{array}{l}T \\
\stackrel{1}{1} \\
\text { i }\end{array}$ & & 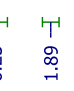 & & & & & & $\begin{array}{l}T \\
+ \\
\dot{0} \\
\text { j }\end{array}$ & & 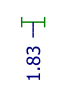 & & & $\begin{array}{l}\text { d. } \\
\text { o. } \\
\text { in }\end{array}$ & & & & \\
\hline 9.5 & 9.0 & 8.5 & 8.0 & 7.5 & 7.0 & 6.5 & 6.0 & 5.5 & 5.0 & $\begin{array}{c}4.5 \\
\text { f1 (ppm) }\end{array}$ & 4.0 & 3.5 & 3.0 & 2.5 & 2.0 & 1.5 & 1.0 & 0.5 & 0.0 & -0.5 \\
\hline
\end{tabular}

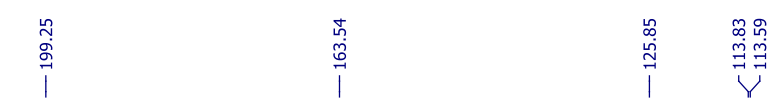

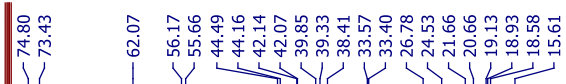

${ }^{13} \mathrm{C}-\mathrm{NMR}\left(126 \mathrm{MHz} \mathrm{CDCl}_{3}\right.$ )<smiles>COc1cccc(C(C)=O)c1</smiles>

$\mathrm{CHCl}_{3}$

\section{Not separable}

from 41

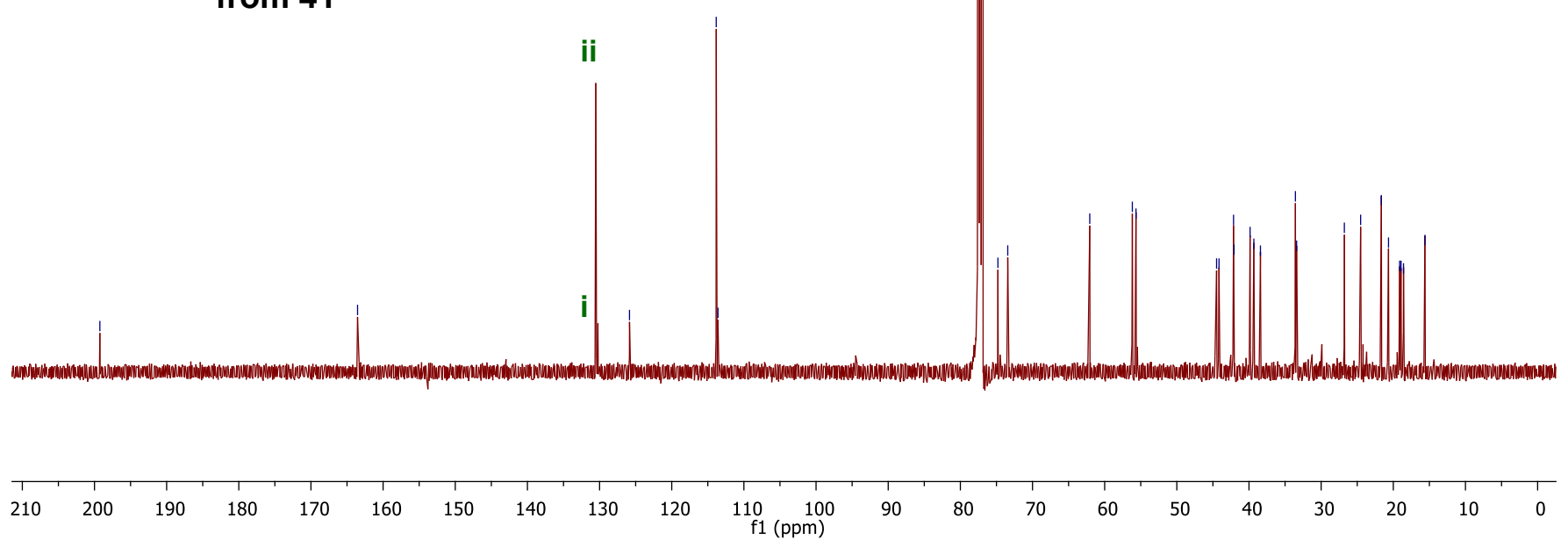


${ }^{1} \mathrm{H}-\mathrm{NMR}$ (300 MHz; $\mathrm{CDCl}_{3}$ )

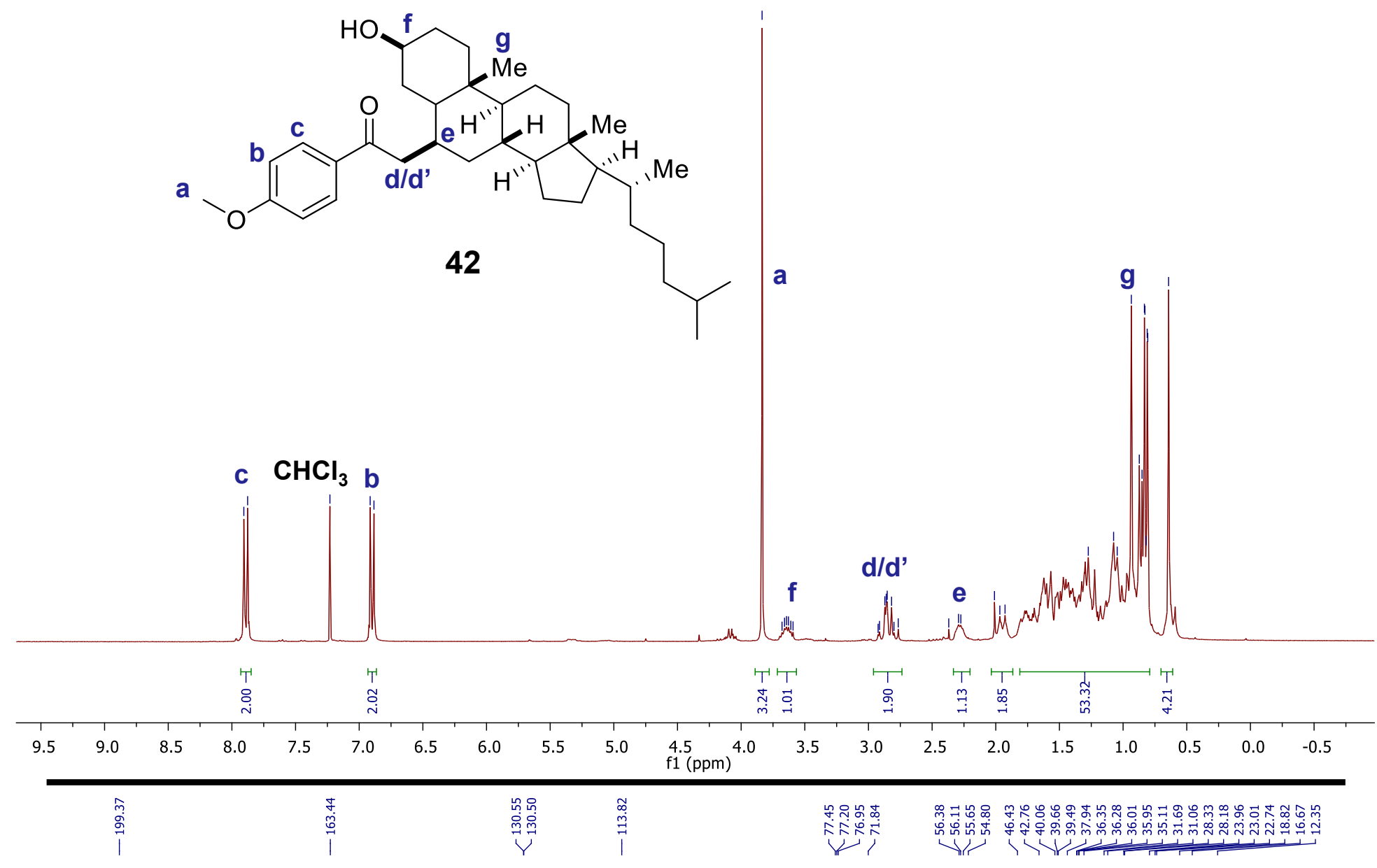

${ }^{13} \mathrm{C}-\mathrm{NMR}$ (126 MHz; $\mathrm{CDCl}_{3}$ )

\section{$\mathrm{CHCl}_{3}$}

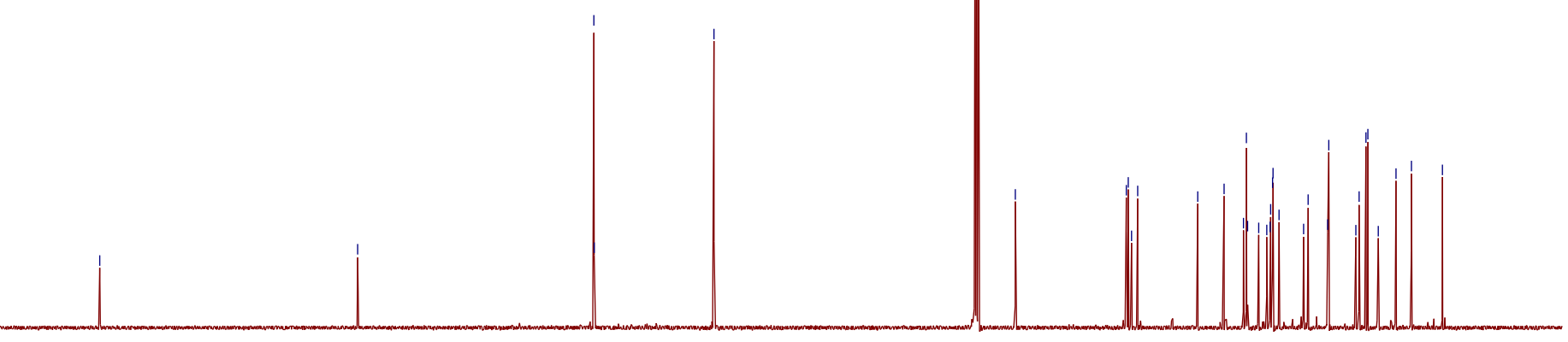

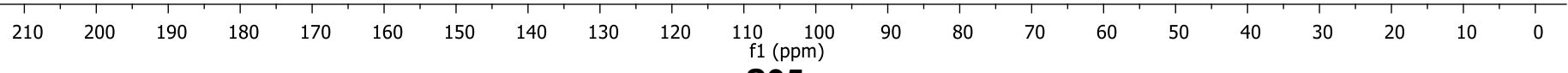


${ }^{1} \mathrm{H}-{ }^{1} \mathrm{H}$ NMR $\left(500 \mathrm{MHz} ; \mathrm{C}_{6} \mathrm{D}_{6}\right) \mathrm{COSY}$

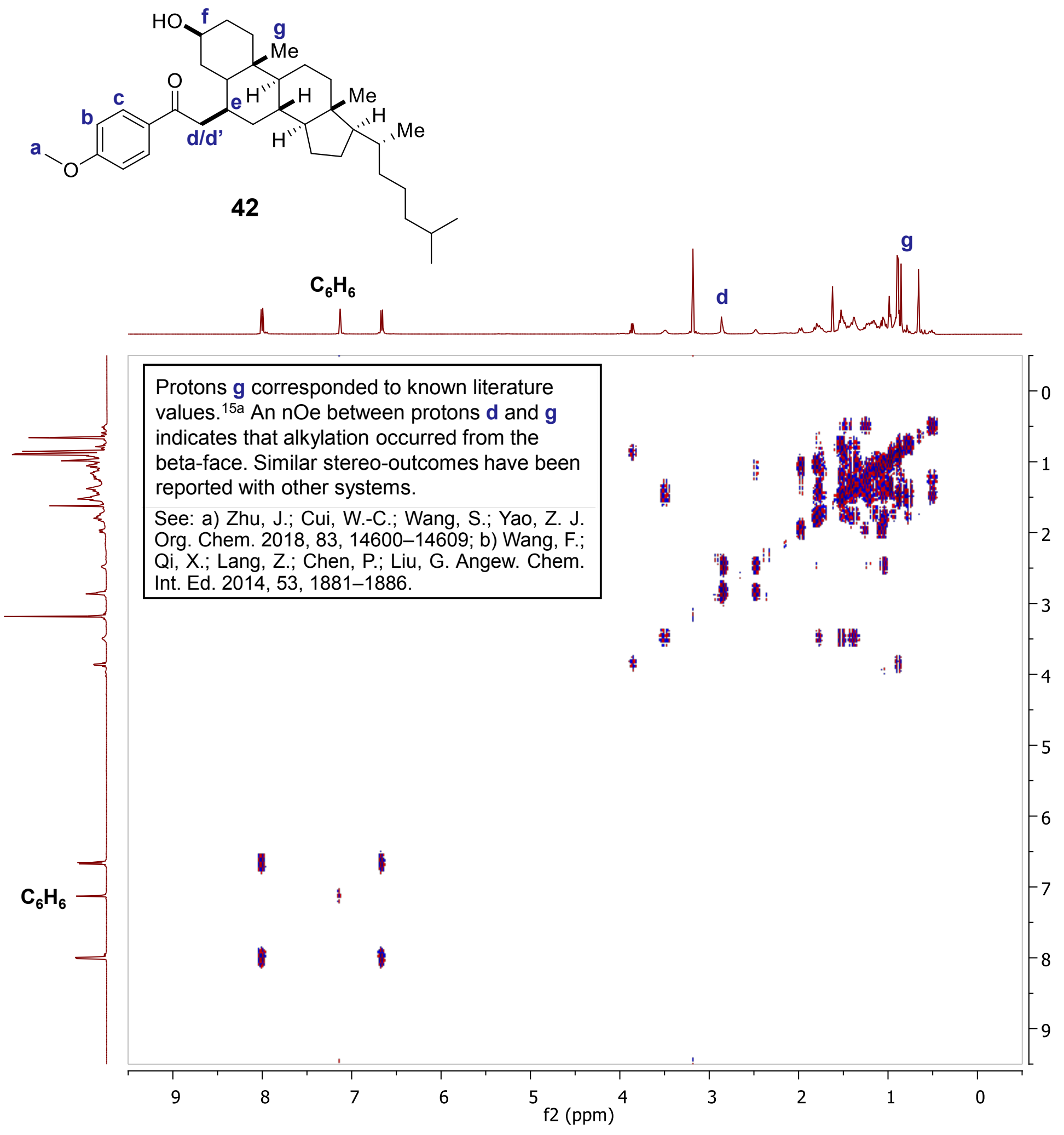


${ }^{1} \mathrm{H}-{ }^{1} \mathrm{H}$ NMR (500 MHz; $\mathrm{C}_{6} \mathrm{D}_{6}$ ) NOESY

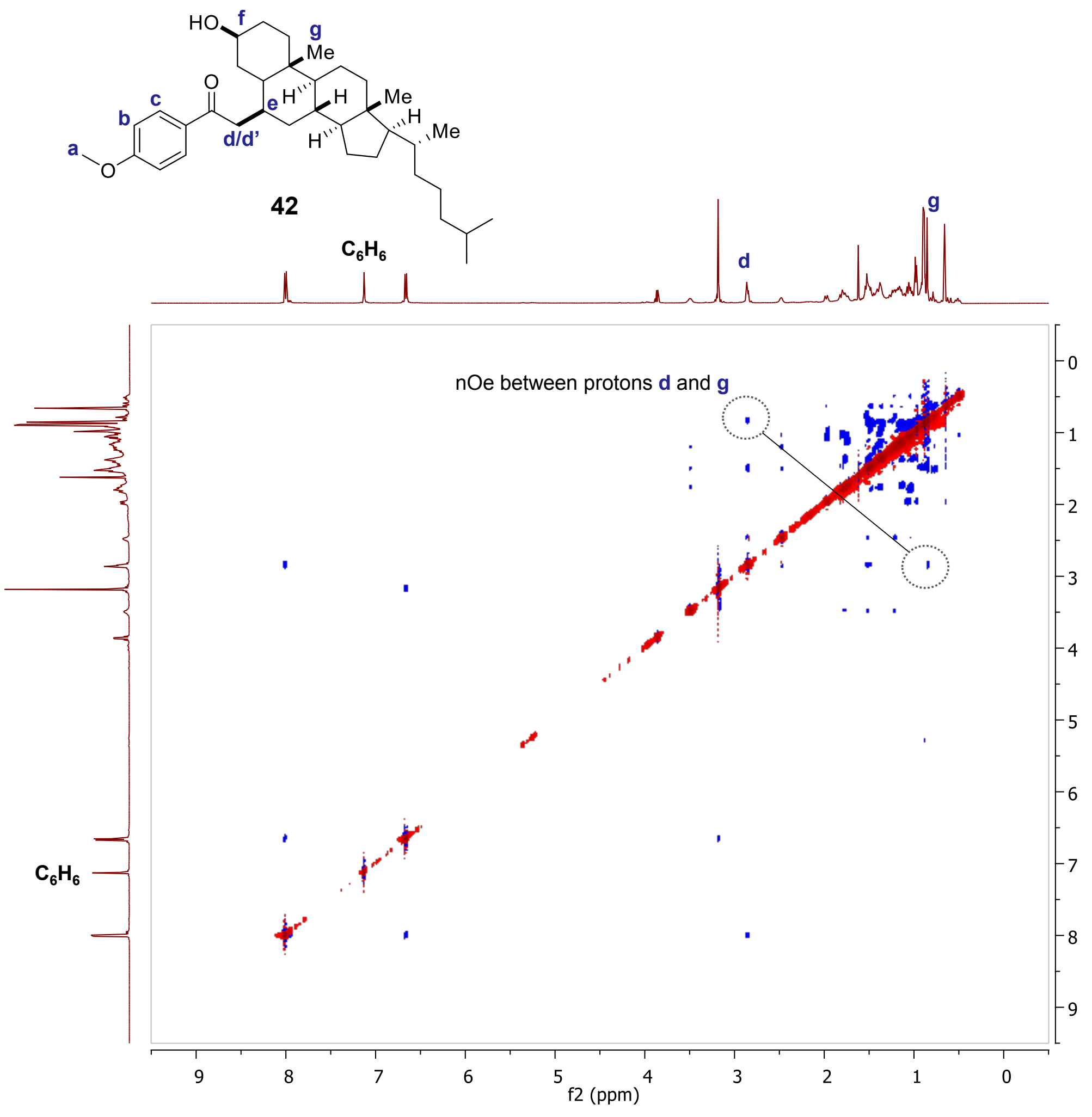


${ }^{1} \mathrm{H}-\mathrm{NMR}\left(300 \mathrm{MHz} ; \mathrm{CDCl}_{3}\right)$

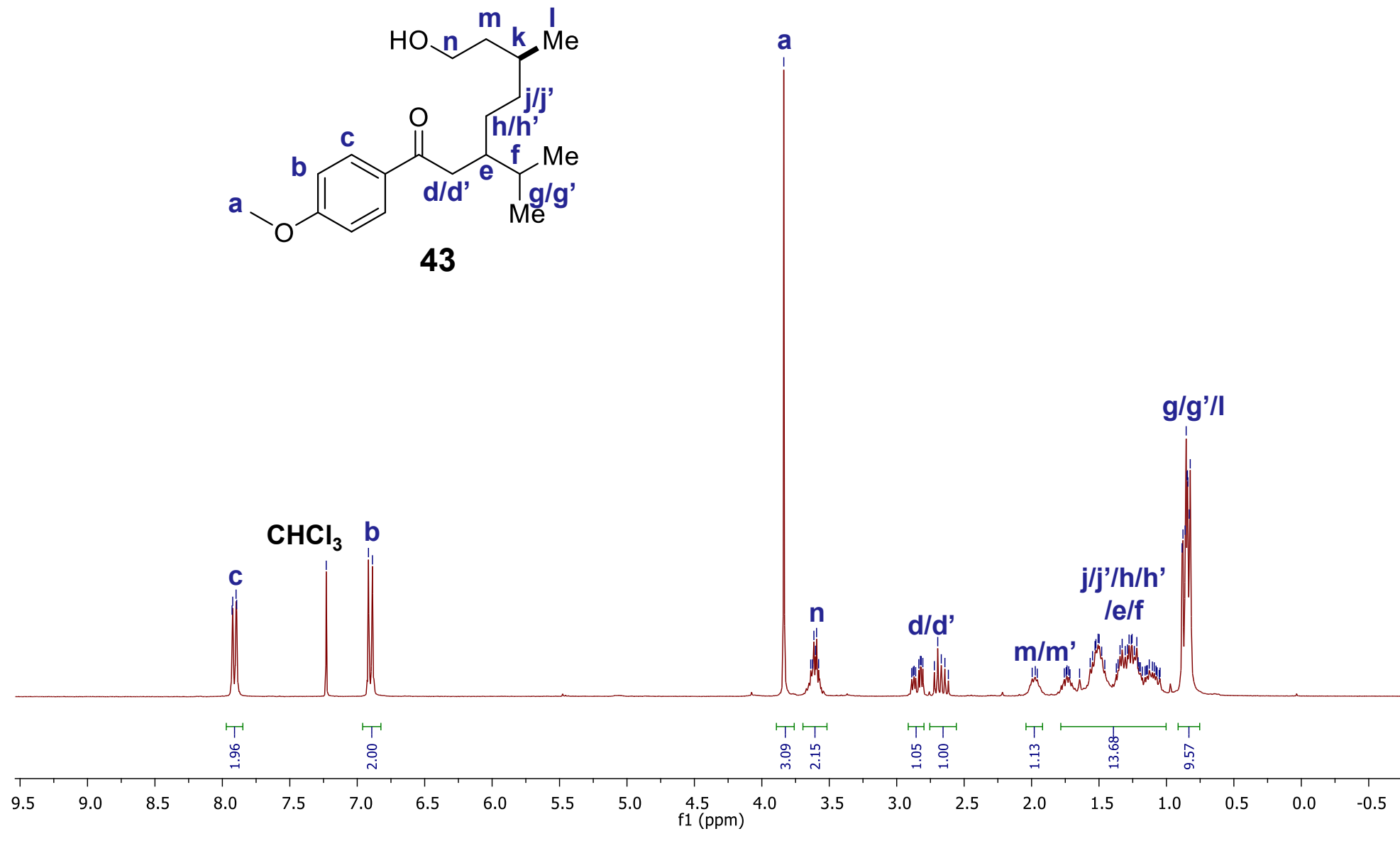

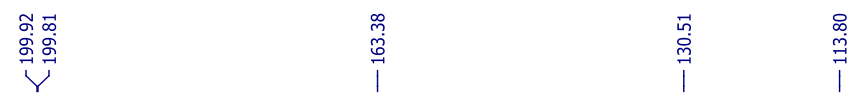

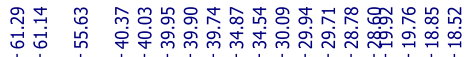

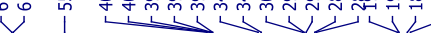

${ }^{13} \mathrm{C}-\mathrm{NMR}\left(126 \mathrm{MHz} ; \mathrm{CDCl}_{3}\right.$ )

$\mathrm{CHCl}_{3}$ 


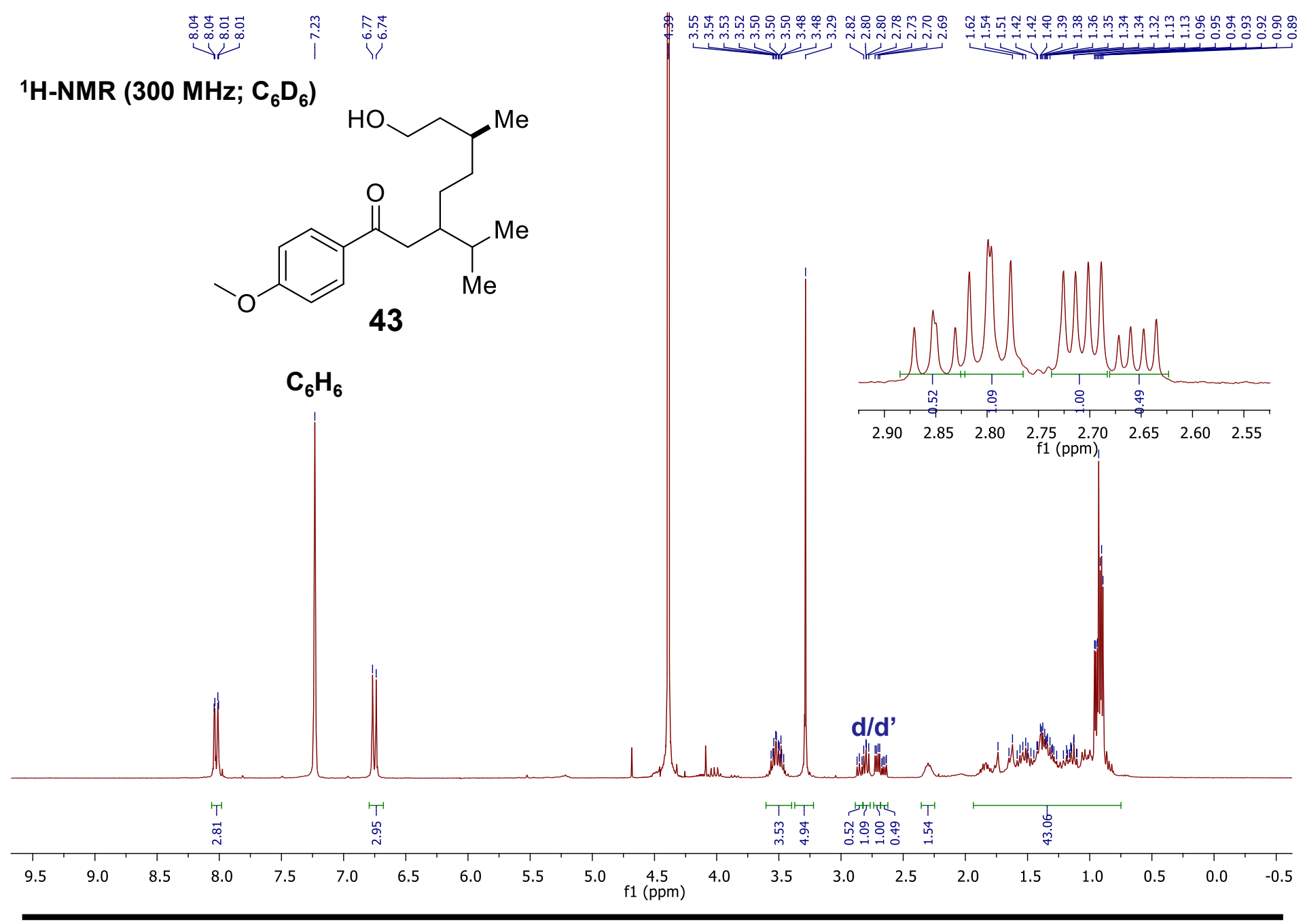

The relative areas of the signals at $2.62 \mathrm{ppm}$ to $2.87 \mathrm{ppm}$ was used to determine the diastereomeric ratio of compound $\mathbf{4 3}$ 
${ }^{1} \mathrm{H}-\mathrm{NMR}\left(500 \mathrm{MHz} ; \mathrm{C}_{6} \mathrm{D}_{6}\right)$

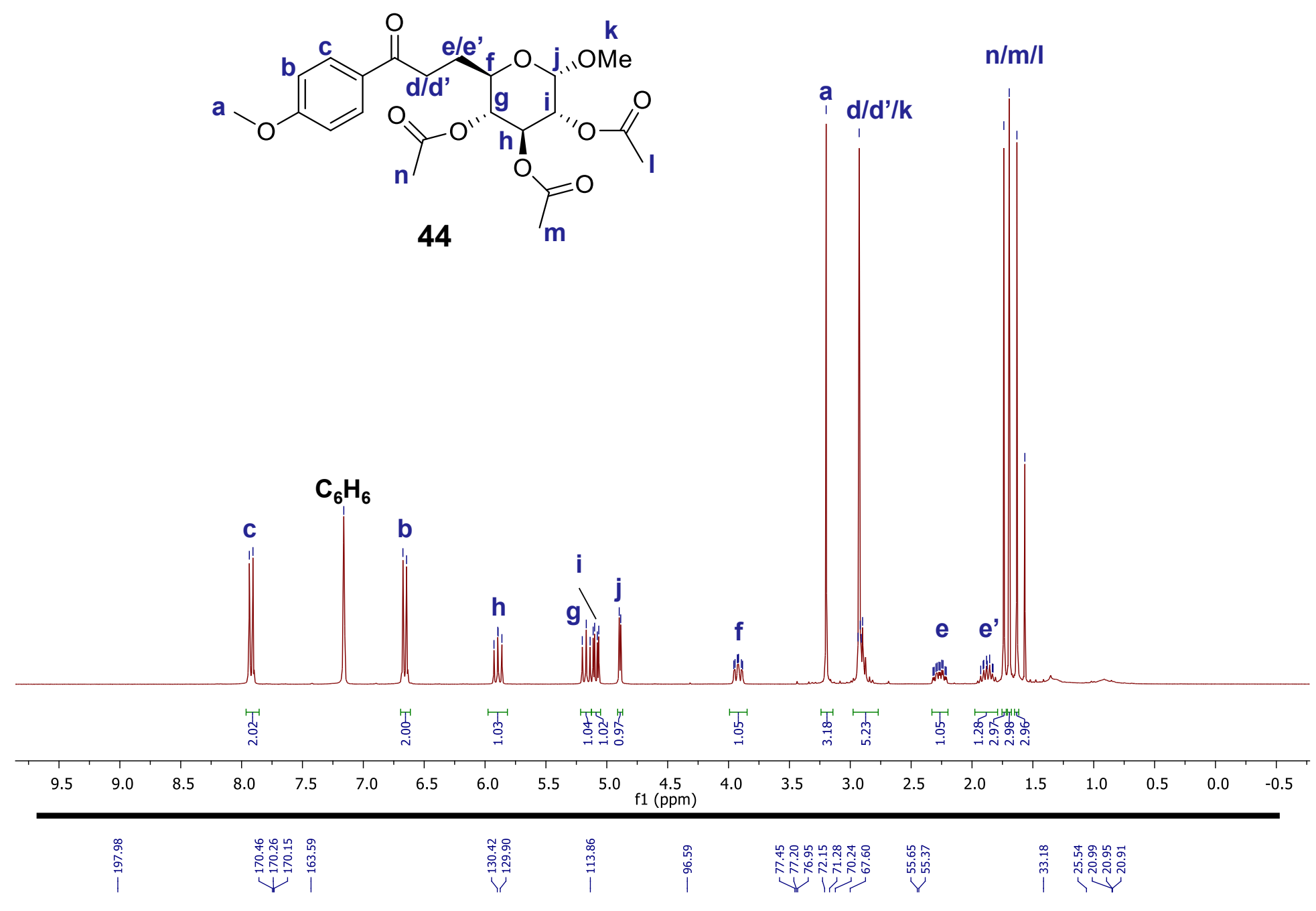

${ }^{13} \mathrm{C}-\mathrm{NMR}\left(126 \mathrm{MHz}^{\mathrm{CDCl}}{ }_{3}\right)$

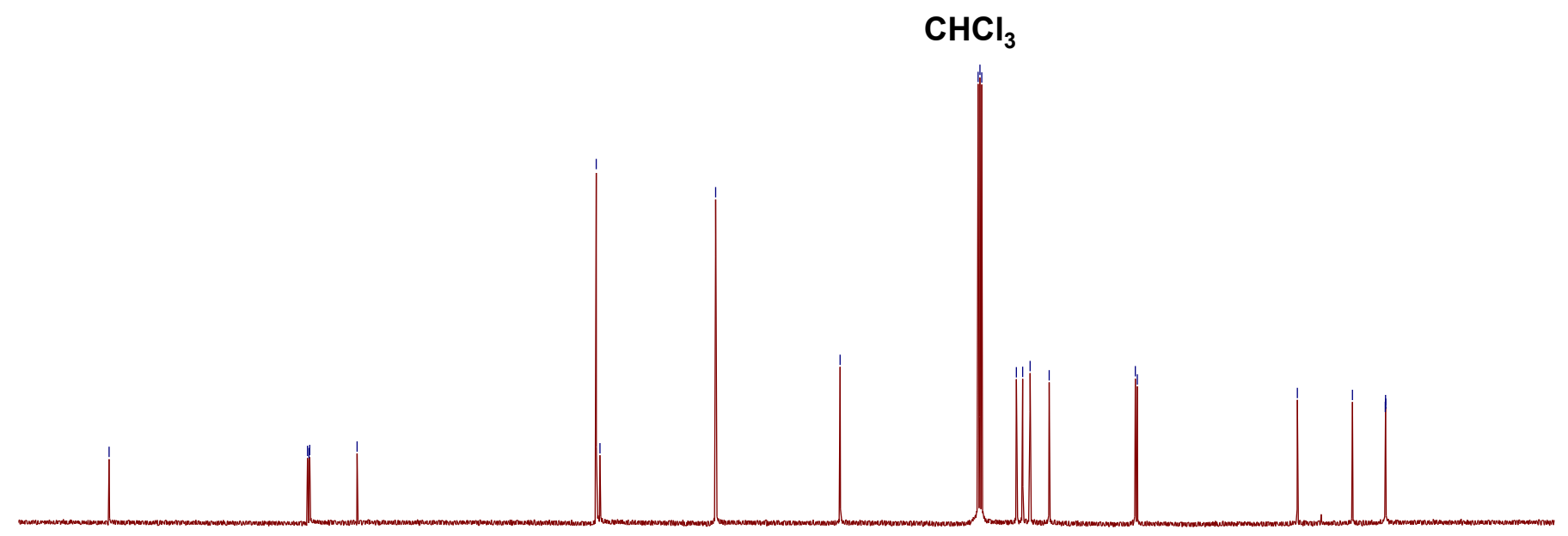

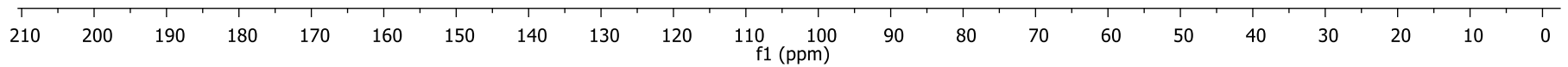



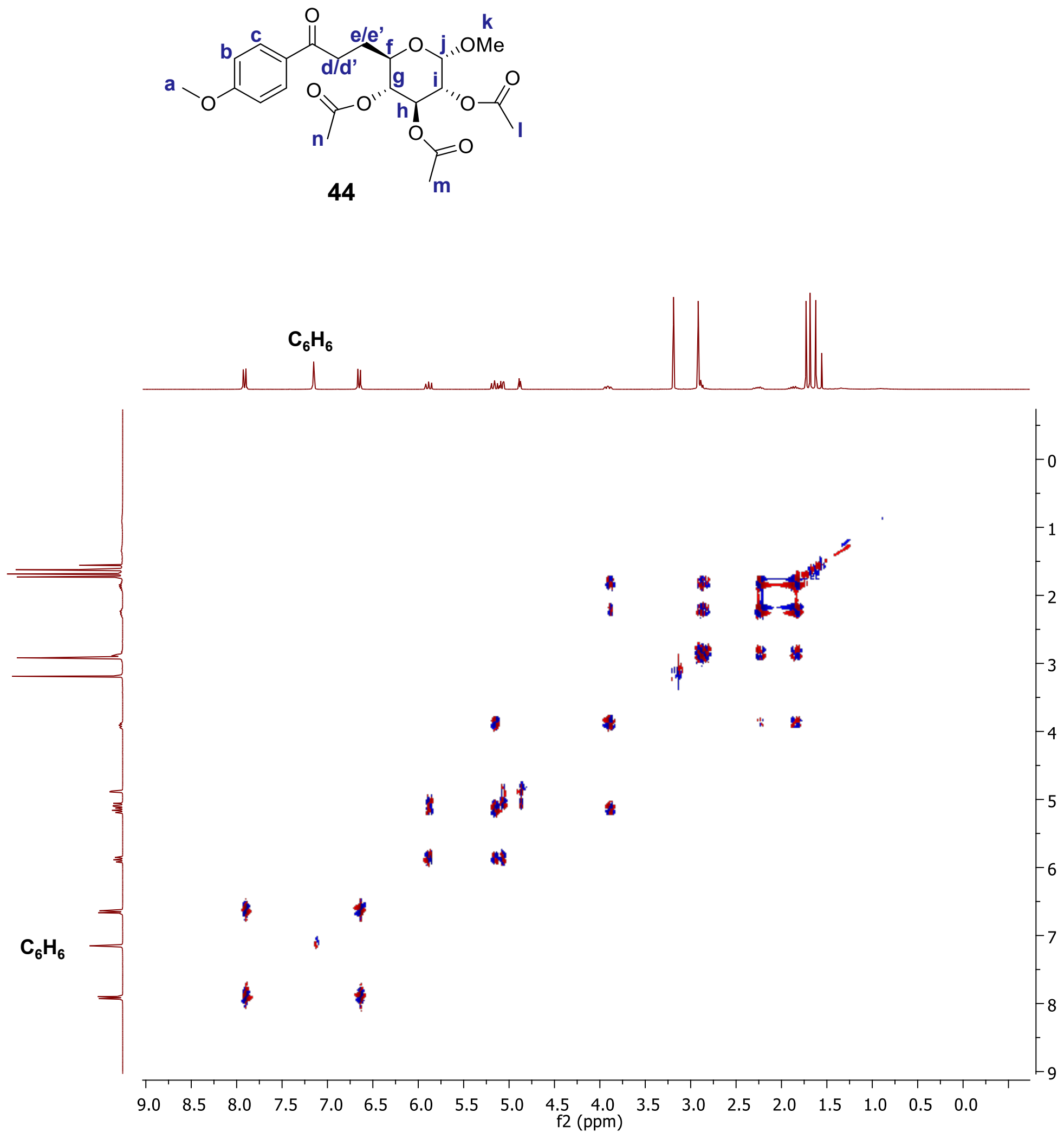


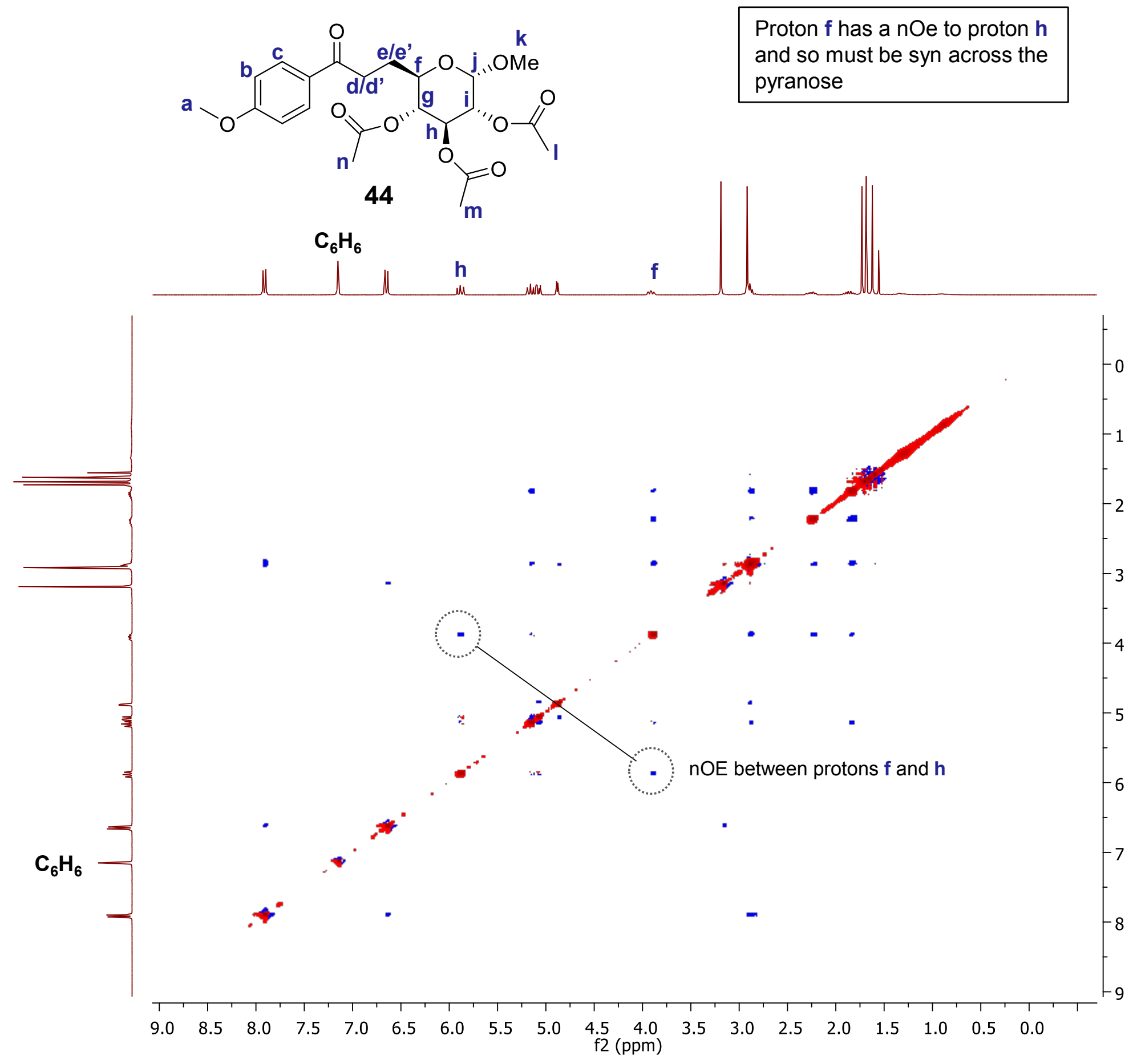


${ }^{1} \mathrm{H}-\mathrm{NMR}\left(300 \mathrm{MHz} ; \mathrm{CDCl}_{3}\right.$ )

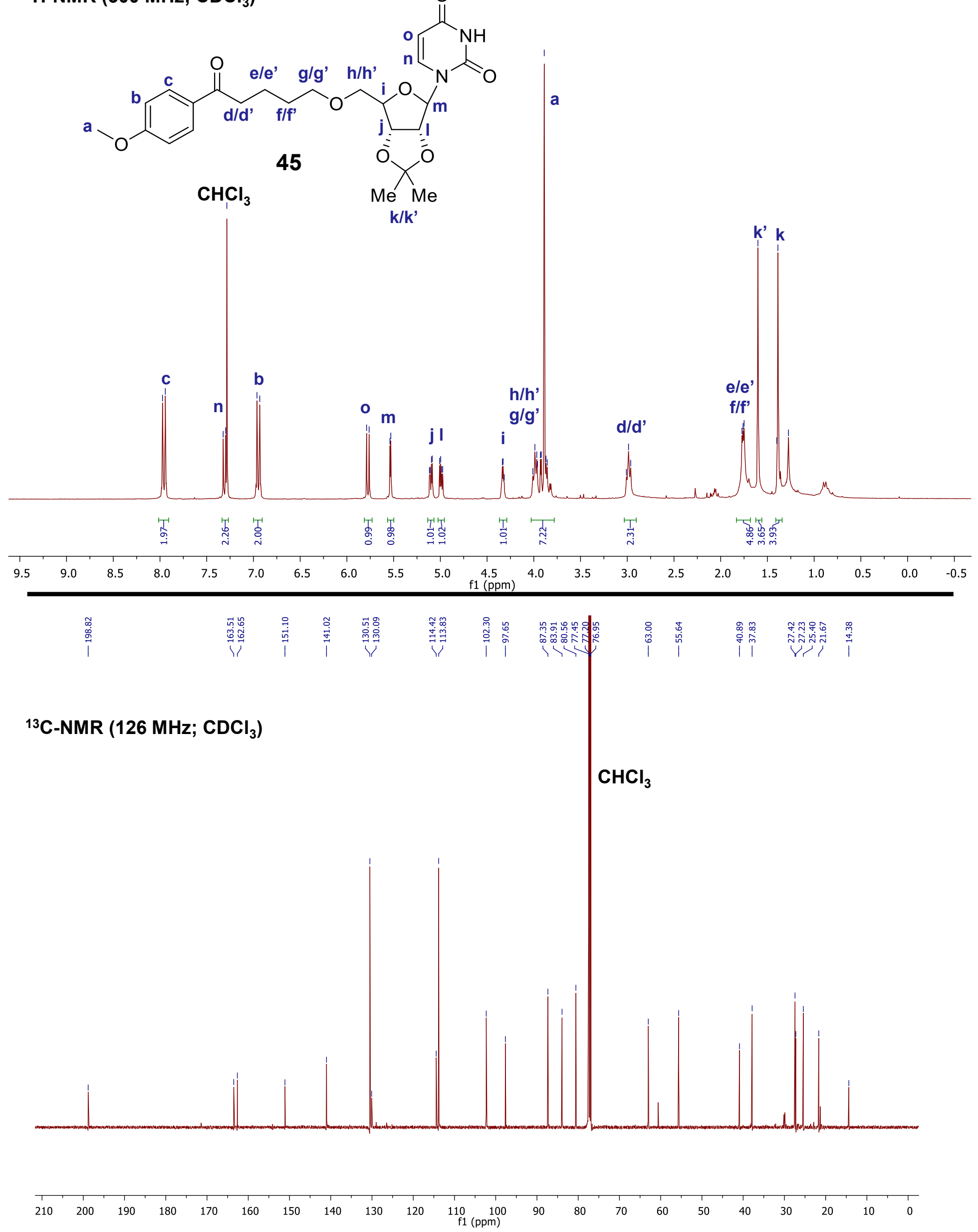


${ }^{1} \mathrm{H}-\mathrm{NMR}\left(300 \mathrm{MHz} ; \mathrm{CDCl}_{3}\right)$<smiles>O=C(CCCOCCCI)c1ccc(F)cc1</smiles>

$46 a$

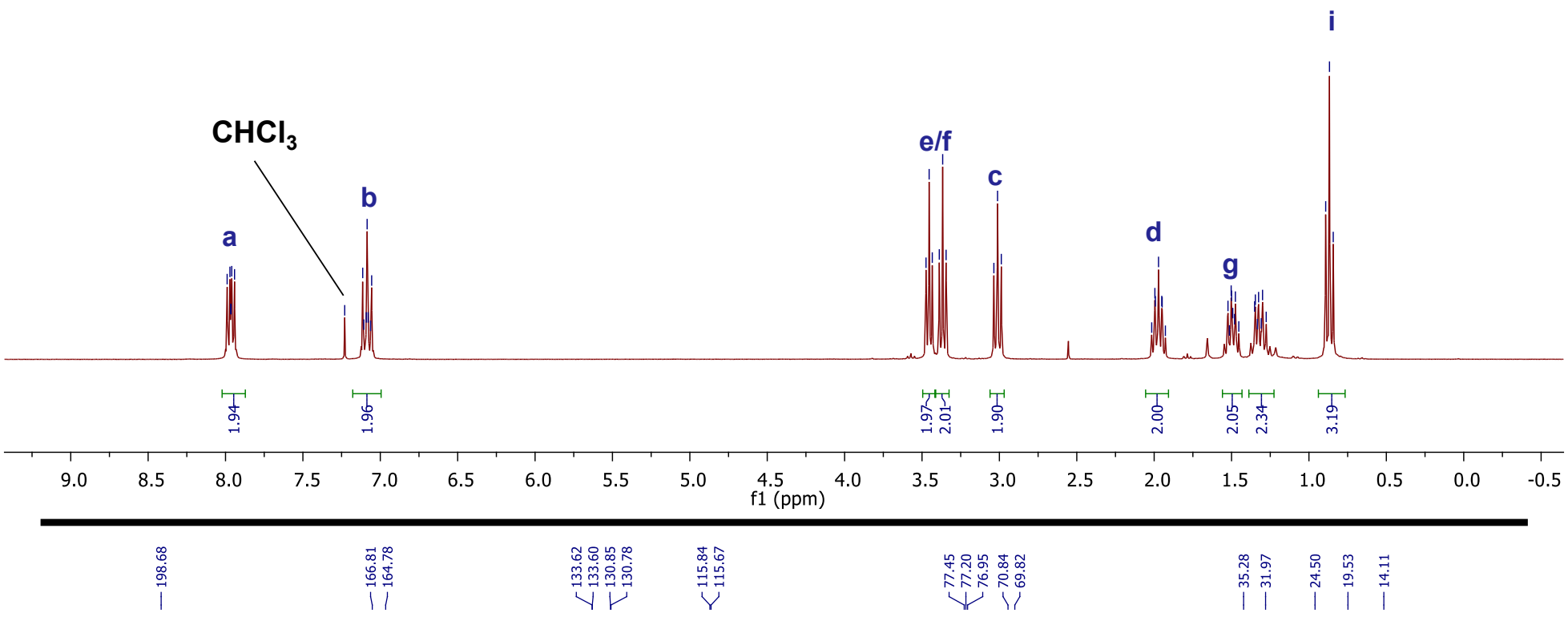

${ }^{13} \mathrm{C}-\mathrm{NMR}\left(126 \mathrm{MHz} \mathrm{CDCl}_{3}\right.$ )

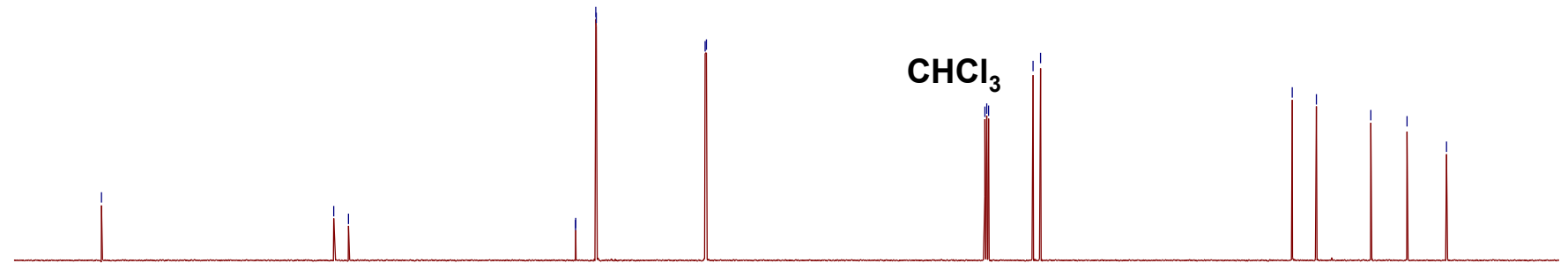

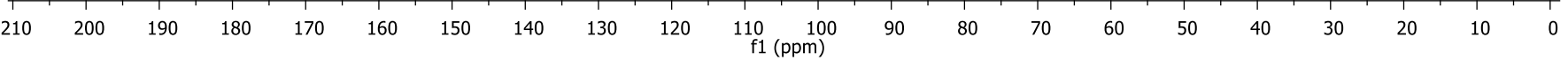


${ }^{1} \mathrm{H}-\mathrm{NMR}$ (300 MHz; $\mathrm{CDCl}_{3}$ )
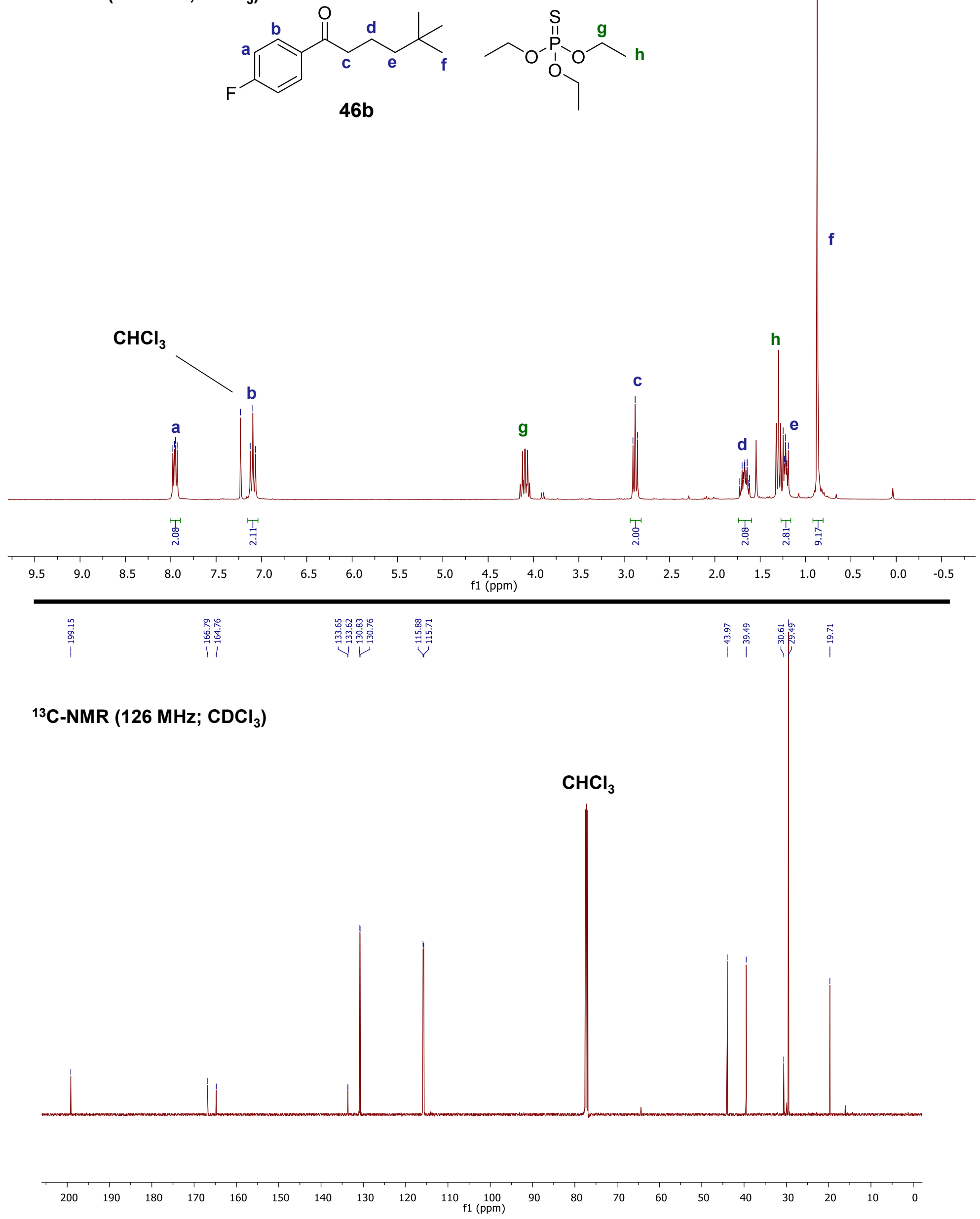

S105 
${ }^{1} \mathrm{H}-\mathrm{NMR}\left(300 \mathrm{MHz} ; \mathrm{CDCl}_{3}\right.$ )<smiles>O=C(CCC(F)OCCC(I)I)c1ccccc1</smiles>

$47 a$

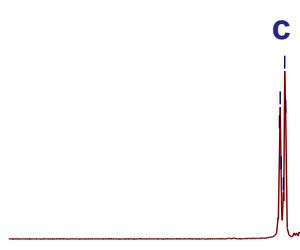

c b

$\mathrm{CHCl}_{3}$

a

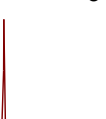

倠

\begin{tabular}{|c|c|c|c|c|c|c|c|c|c|c|}
\hline 9.0 & 8.5 & 8.0 & 7.5 & 7.0 & 6.5 & 6.0 & 5.5 & 5.0 & $\begin{array}{l}4.5 \\
\text { f1 (ppm) }\end{array}$ & 4.0 \\
\hline
\end{tabular}
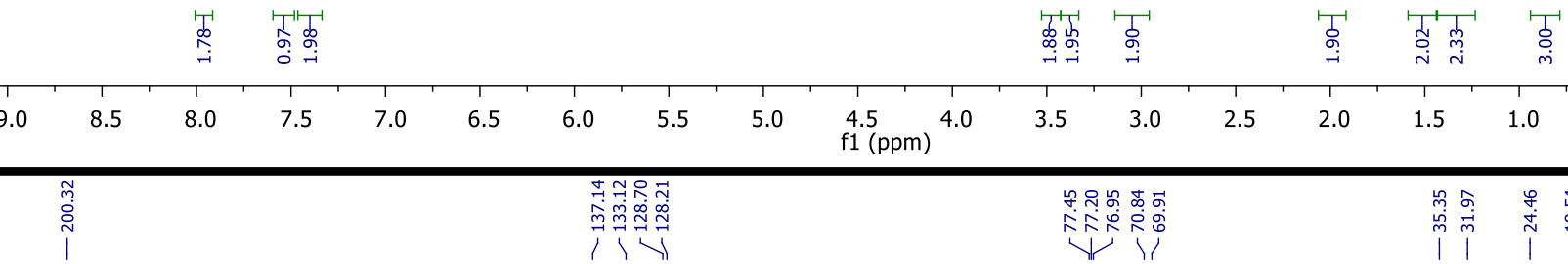

i

군

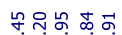

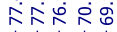

$\backslash \backslash$



${ }^{13} \mathrm{C}-\mathrm{NMR}\left(126 \mathrm{MHz} ; \mathrm{CDCl}_{3}\right.$ )

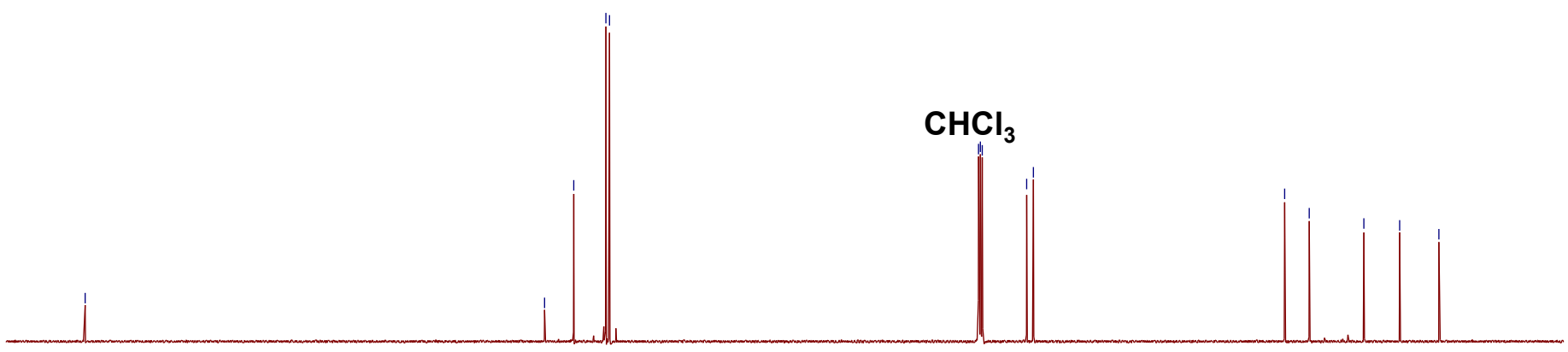


${ }^{1} \mathrm{H}-\mathrm{NMR}$ (300 MHz; $\mathrm{CDCl}_{3}$ )<smiles>CC(C)(C)[C]C[C]C(=O)c1ccccc1</smiles>

47b

d
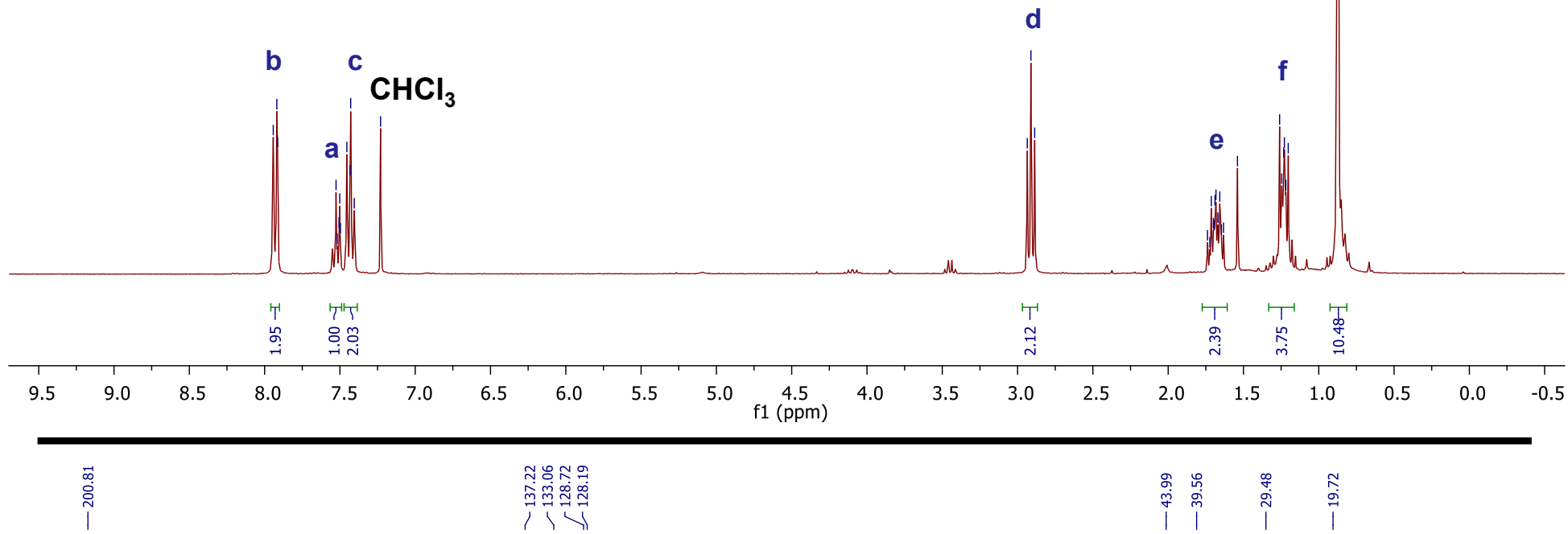

๙

${ }^{13} \mathrm{C}-\mathrm{NMR}\left(126 \mathrm{MHz} ; \mathrm{CDCl}_{3}\right.$ )

$\mathrm{CHCl}_{3}$
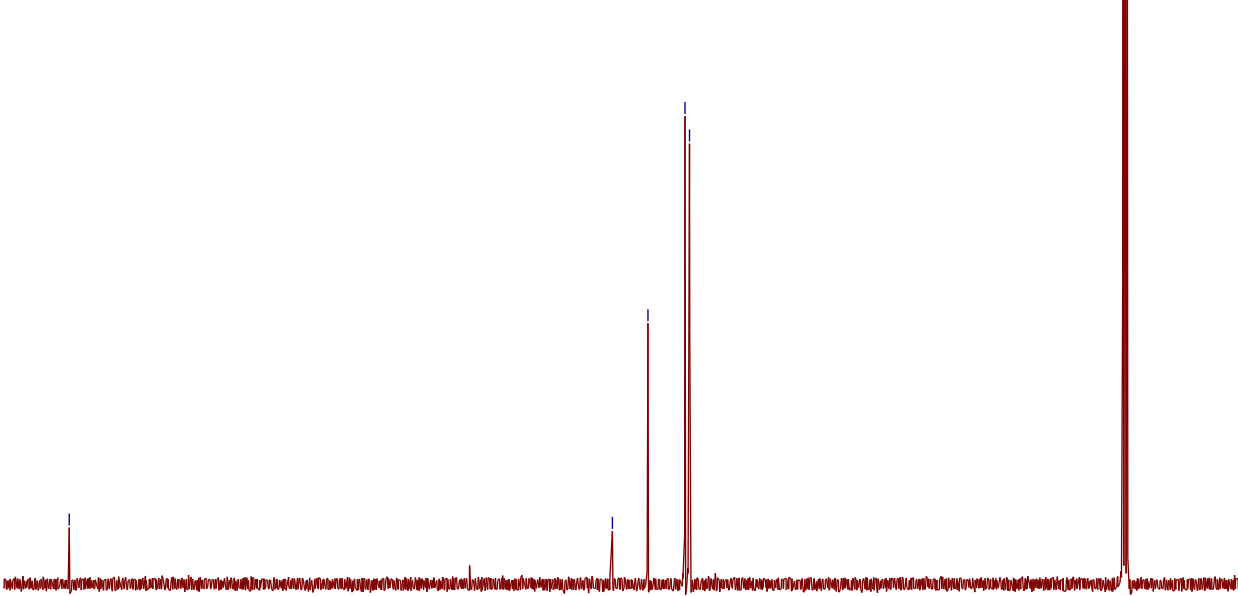

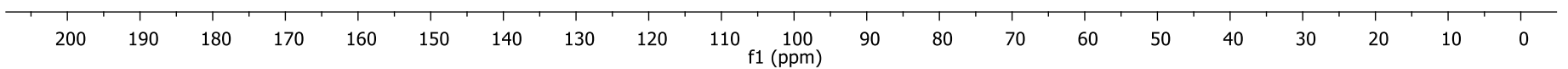


${ }^{1} \mathrm{H}-\mathrm{NMR}$ (300 MHz; $\mathrm{CDCl}_{3}$ )

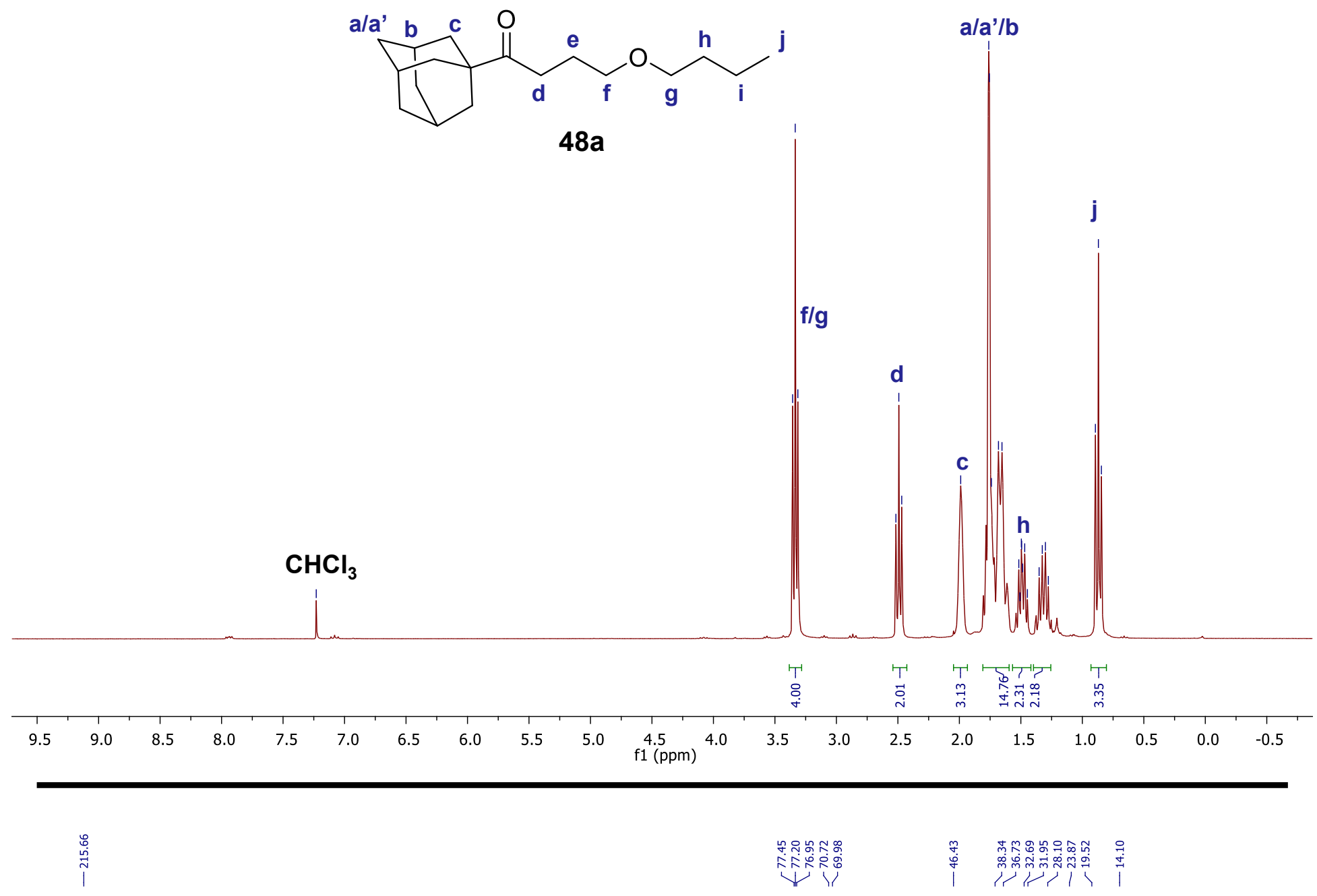

${ }^{13}$ C-NMR (126 MHz; $\mathrm{CDCl}_{3}$ )

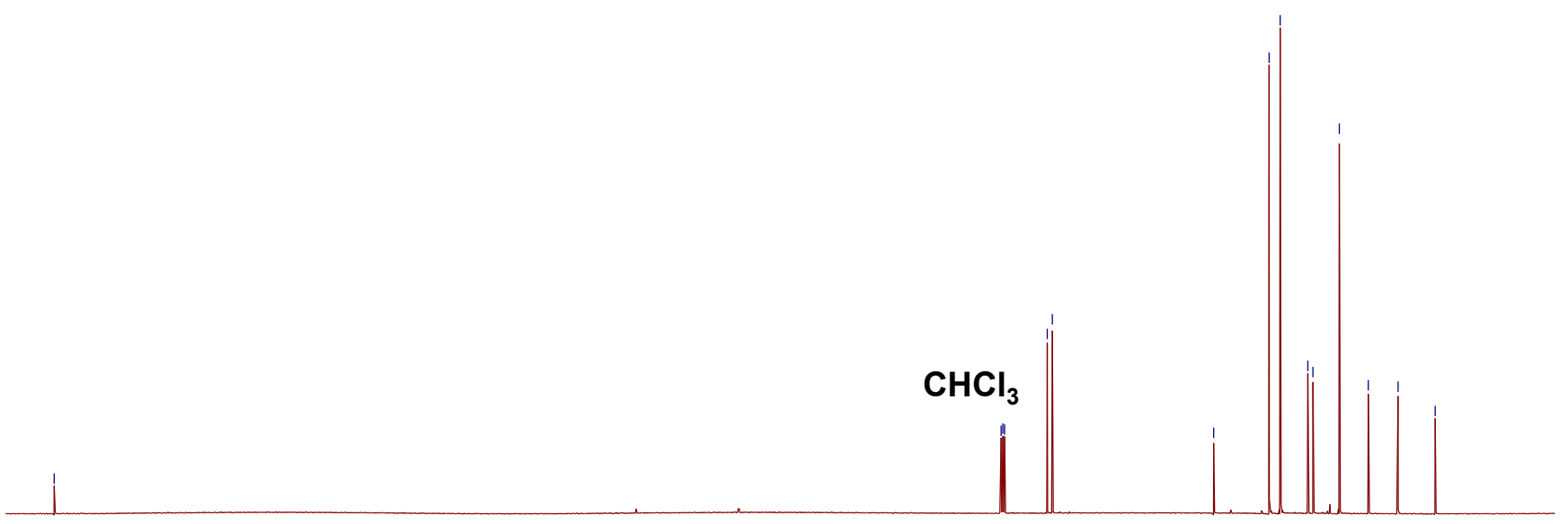

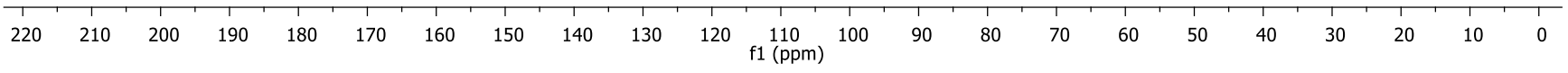


${ }^{1} \mathrm{H}-\mathrm{NMR}$ (300 MHz; $\mathrm{CDCl}_{3}$ )

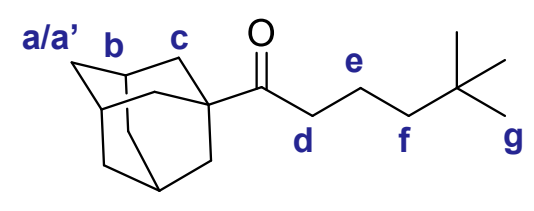

$48 b$<smiles>CCOP(=S)(OCC)OCC</smiles>

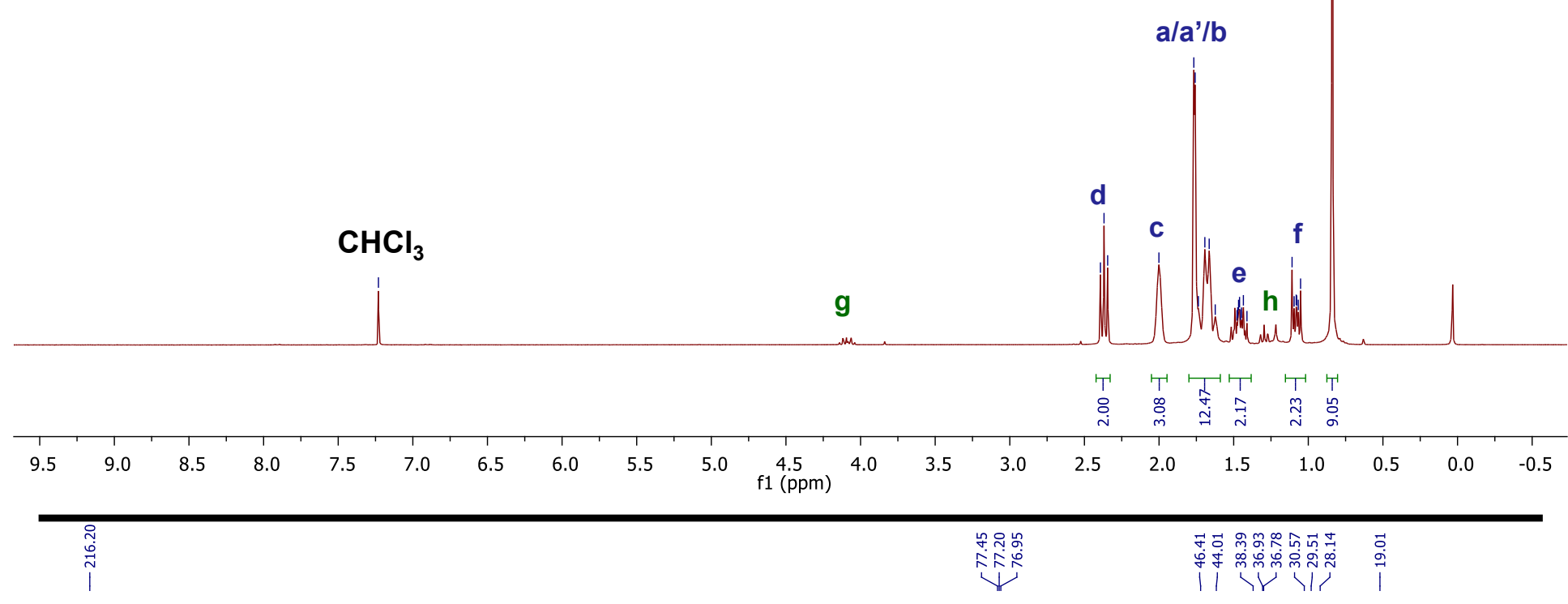

${ }^{13} \mathrm{C}-\mathrm{NMR}$ (126 MHz; $\mathrm{CDCl}_{3}$ )
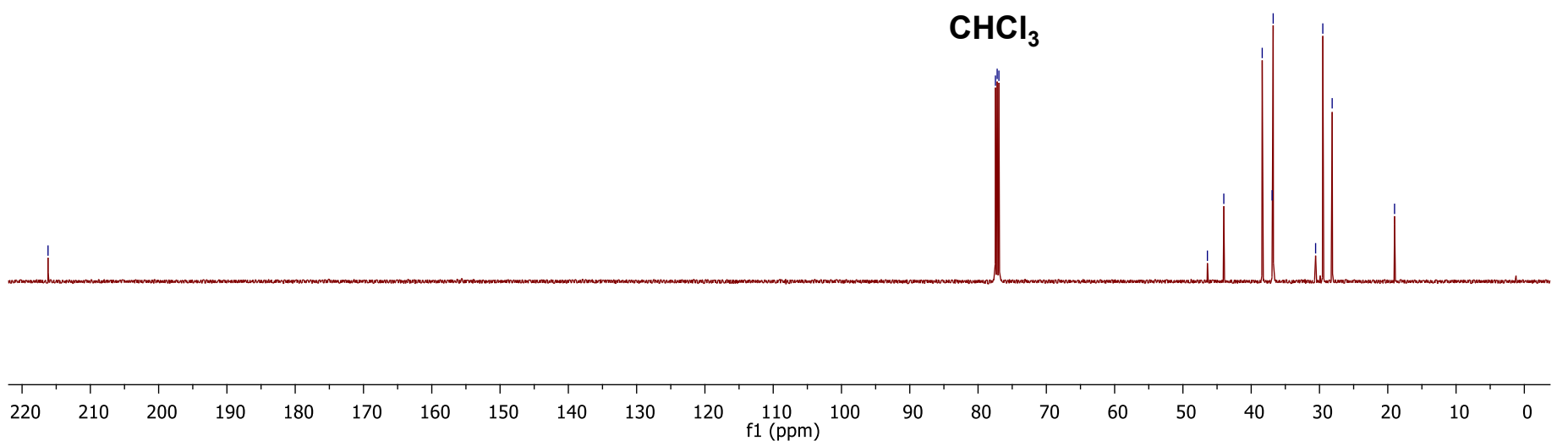
${ }^{1} \mathrm{H}-\mathrm{NMR}\left(300 \mathrm{MHz} ; \mathrm{CDCl}_{3}\right.$ )
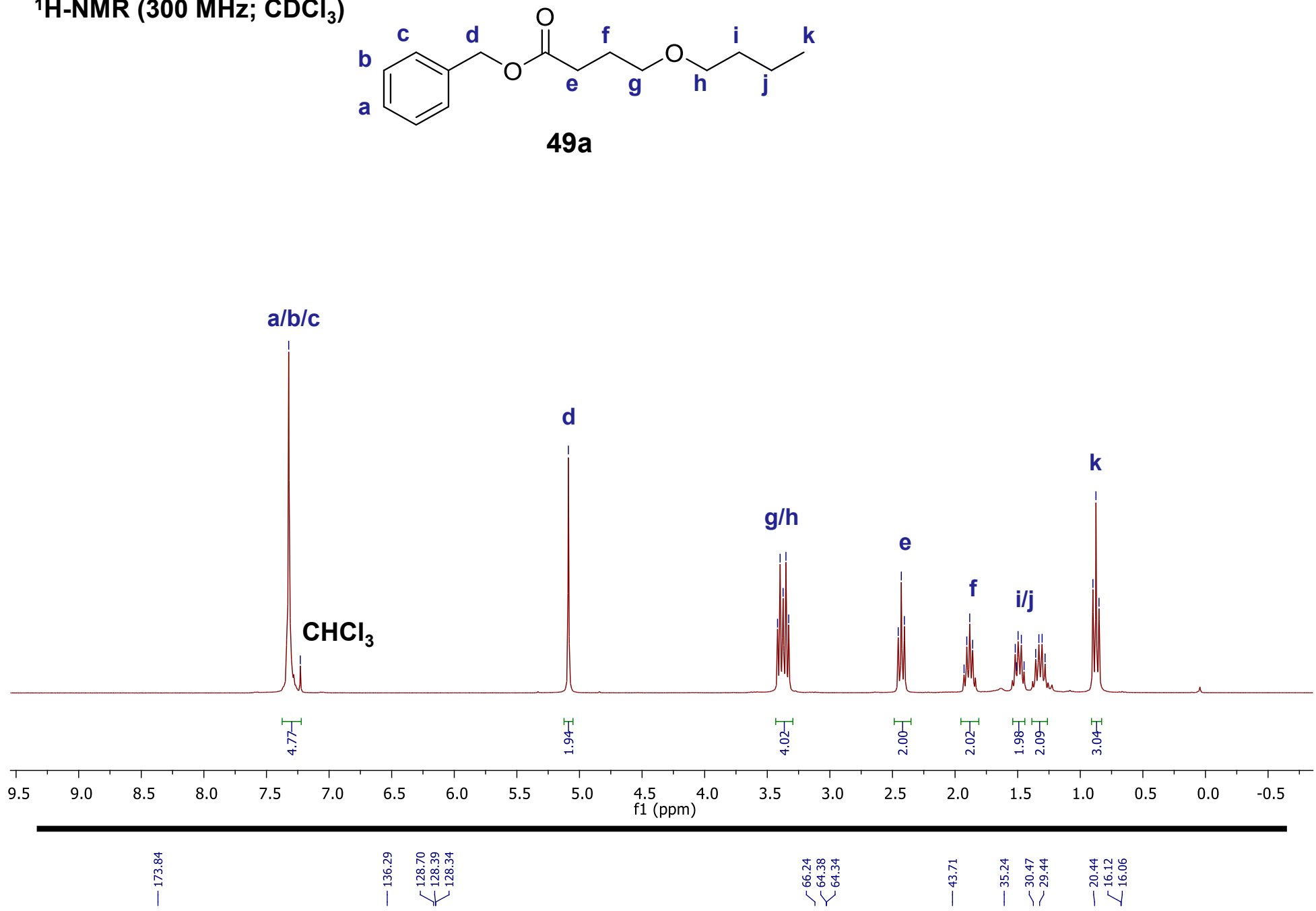

${ }^{13} \mathrm{C}-\mathrm{NMR}\left(126 \mathrm{MHz} ; \mathrm{CDCl}_{3}\right.$ )

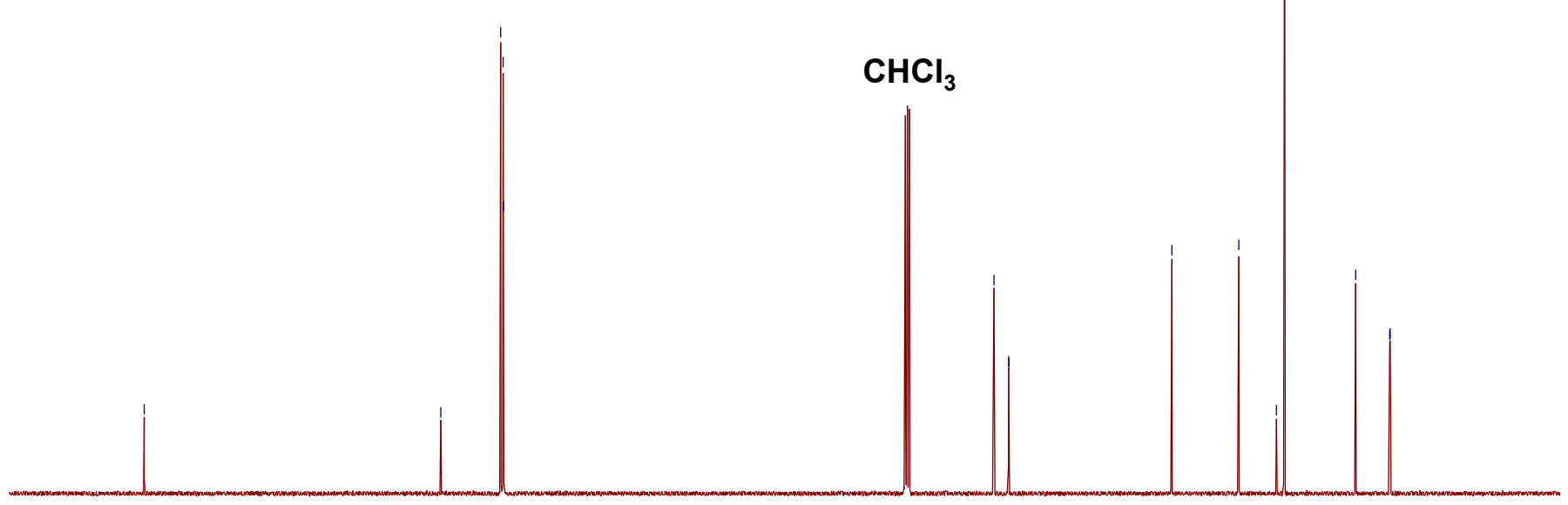

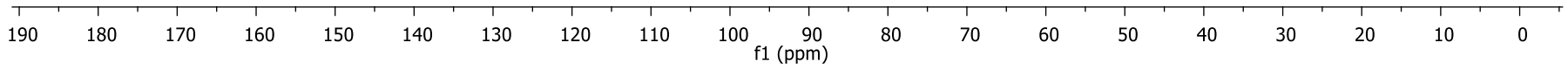




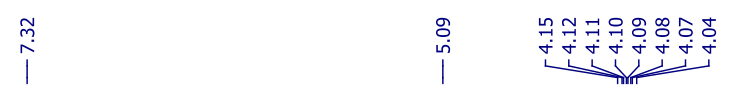

${ }^{1} \mathrm{H}-\mathrm{NMR}\left(300 \mathrm{MHz} ; \mathrm{CDCl}_{3}\right.$ )<smiles>CC(C)(C)[C]CCC(=O)OCc1ccccc1</smiles>

$49 b$<smiles>CCOP(=S)(OCC)O[Hg]C</smiles>

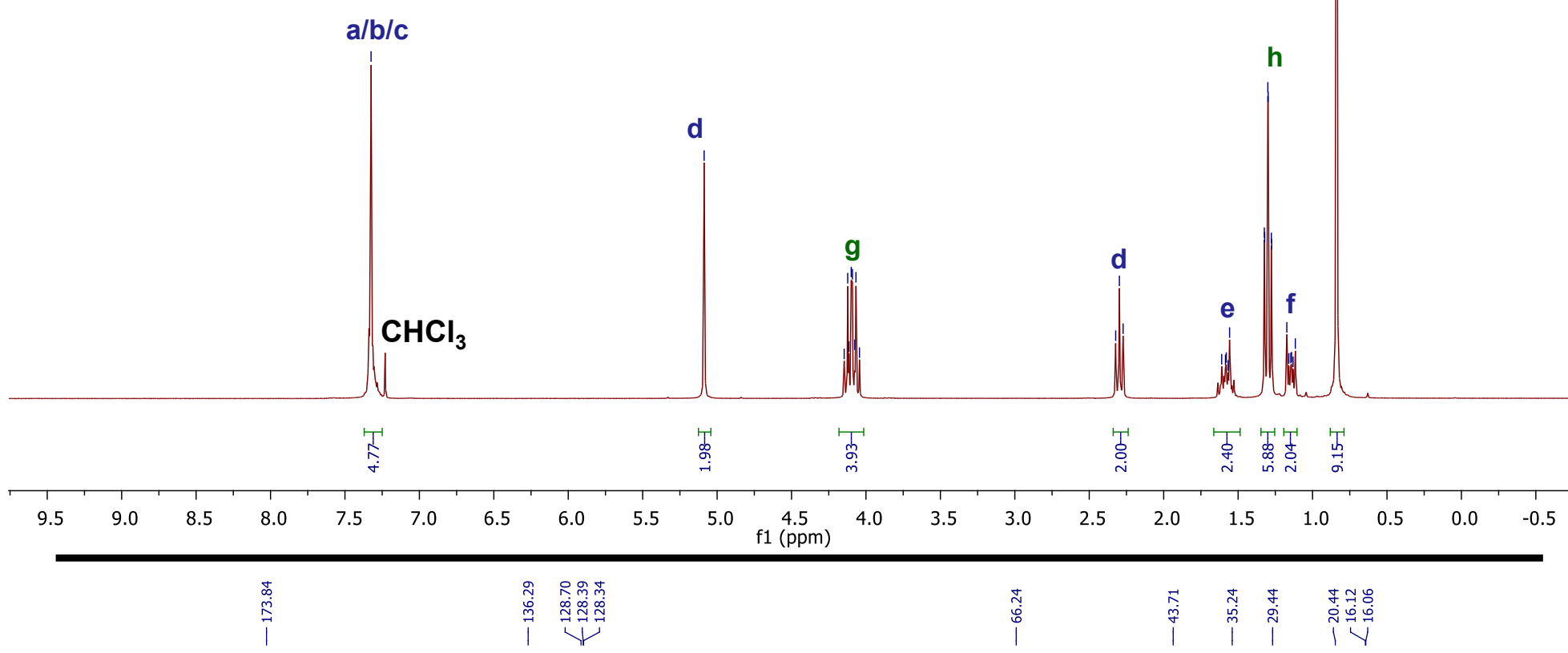

${ }^{13} \mathrm{C}-\mathrm{NMR}$ (126 MHz; $\mathrm{CDCl}_{3}$ )

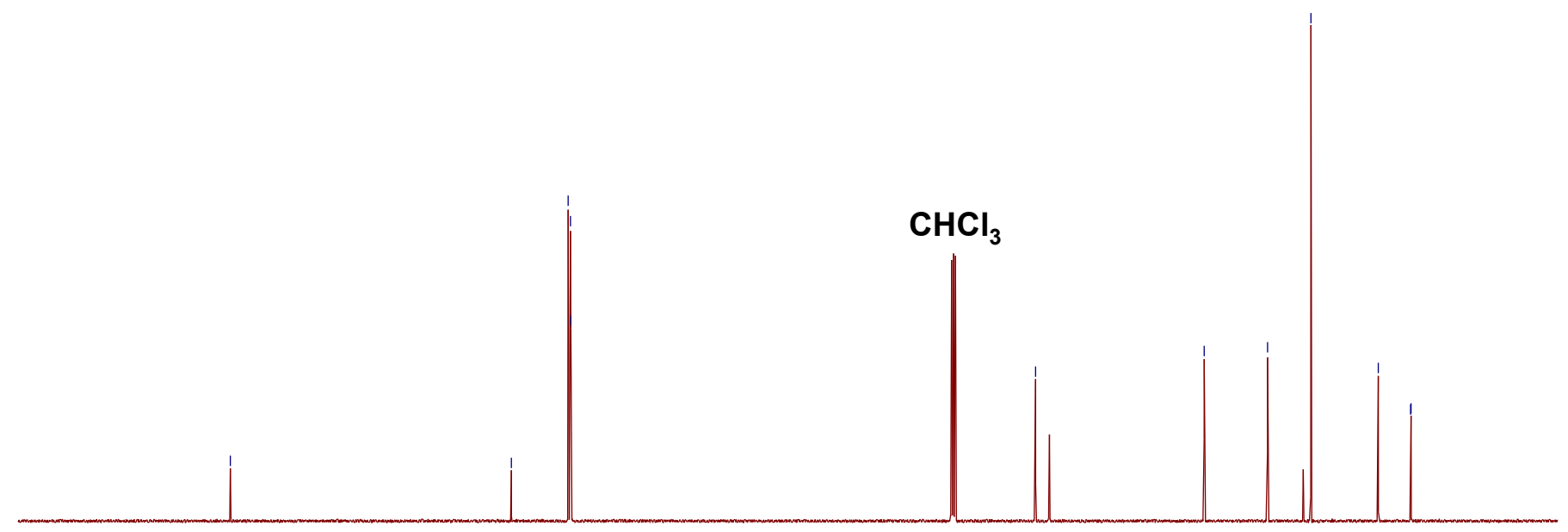

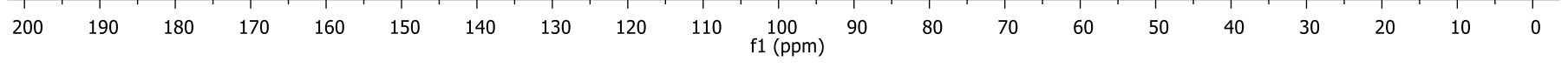


${ }^{1} \mathrm{H}-\mathrm{NMR}\left(300 \mathrm{MHz} ; \mathrm{CDCl}_{3}\right)$<smiles>CCC(C)COCCCP(=O)(OCC(F)(F)F)OC(F)(F)F</smiles>

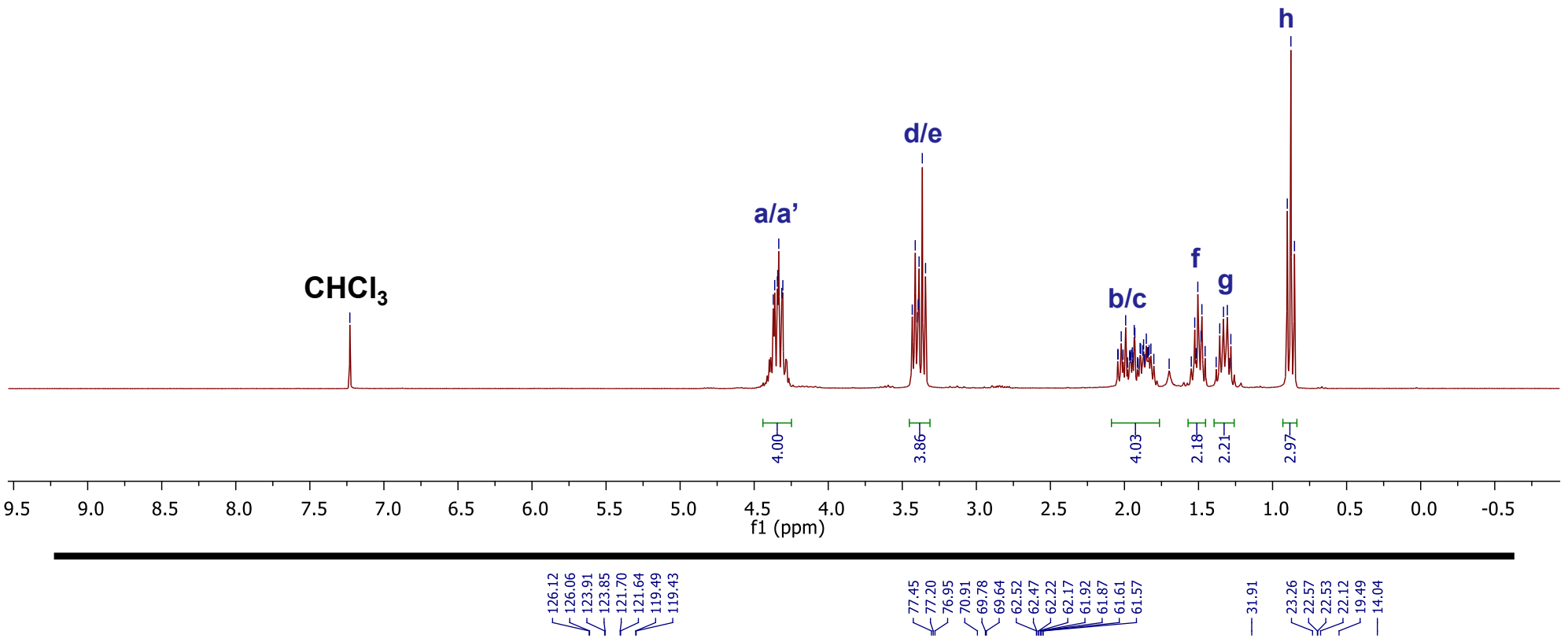

${ }^{13} \mathrm{C}-\mathrm{NMR}\left(126 \mathrm{MHz} ; \mathrm{CDCl}_{3}\right.$ )

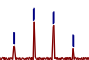

\section{$\mathrm{CHCl}_{3}$}

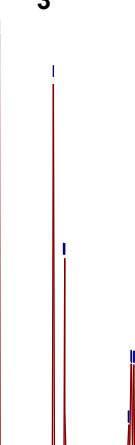

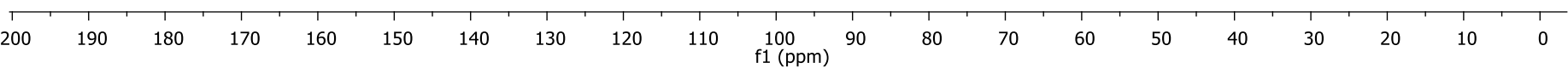


${ }^{19}$ F NMR $(282 \mathrm{~Hz}, \mathrm{CDCl} 3)$<smiles>CCCCOCCCP(=O)(OCC(F)(F)F)OCC(F)(F)F</smiles>

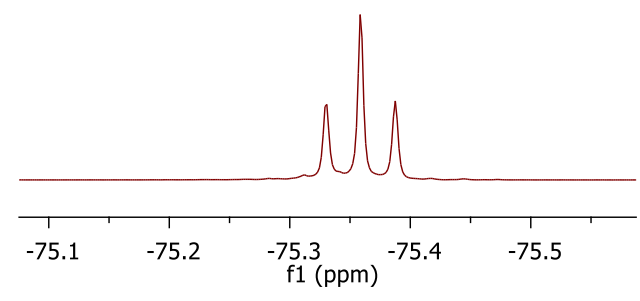

\begin{tabular}{|c|c|c|c|c|c|c|c|c|c|c|c|c|c|c|c|c|c|c|c|c|}
\hline$T$ & 1 & 1 & 1 & 1 & 1 & 1 & $T$ & $T$ & $T$ & 1 & 1 & $T$ & $T$ & $T$ & $T$ & $T$ & $T$ & 1 & $T$ & $T$ \\
\hline-20 & -25 & -30 & -35 & -40 & -45 & -50 & -55 & -60 & -65 & $\begin{array}{l}-70 \\
\mathrm{f} 1 \text { (ppm) }\end{array}$ & -75 & -80 & -85 & -90 & -95 & -100 & -105 & -110 & -115 & $-12 C$ \\
\hline
\end{tabular}

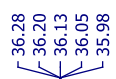

${ }^{31} \mathrm{P}$ NMR (212 MHz, CDCl3)

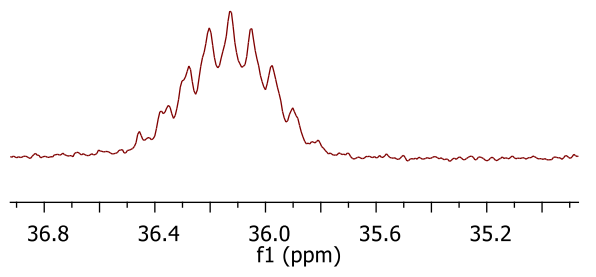

\begin{tabular}{|c|c|c|c|c|c|c|c|c|c|c|c|c|c|c|c|c|c|}
\hline 11 & 1 & $T$ & $T$ & 1 & $T$ & 1 & $T$ & 1 & 1 & 1.1 & 1 & 1 & 1 & 11 & 1 & 1 & 1 \\
\hline 140 & 120 & 100 & 80 & 60 & 40 & 20 & 0 & -10 & -30 & $\begin{array}{c}-50 \\
\mathrm{f} 1(\mathrm{ppm})\end{array}$ & -70 & -90 & -110 & -140 & -170 & -200 & -230 \\
\hline
\end{tabular}


${ }^{1} \mathrm{H}-\mathrm{NMR}$ (300 MHz; $\mathrm{CDCl}_{3}$ )

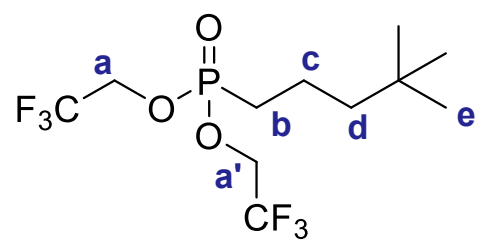

$50 \mathrm{~b}$

e

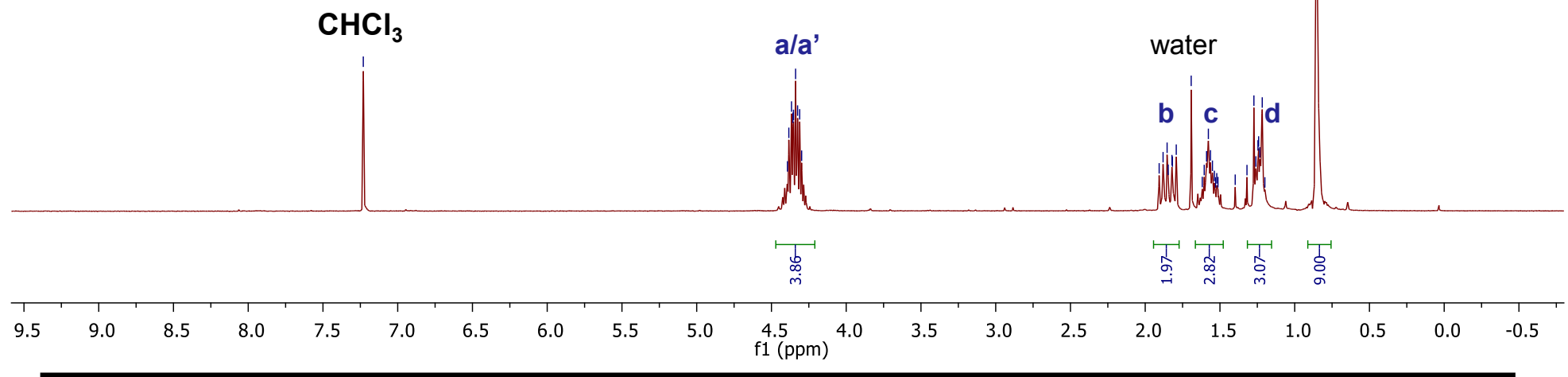

${ }^{13} \mathrm{C}-\mathrm{NMR}$ (126 MHz; $\mathrm{CDCl}_{3}$ )

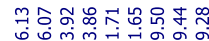

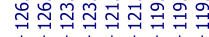

$\longrightarrow$ Y

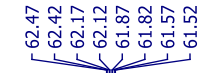

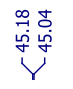

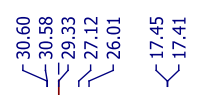

$\mathrm{CHCl}_{3}$ 
${ }^{19}$ F NMR (282 MHz, CDCI3)<smiles>CC(C)(C)CCCP(=O)(OCC(F)(F)F)OCC(F)(F)F</smiles>

\begin{tabular}{|c|c|c|c|c|c|c|c|c|c|c|c|c|c|c|}
\hline-69.5 & -70.5 & -71.5 & -72.5 & -73.5 & -74.5 & -75.5 & $\begin{array}{l}-76.5 \\
\text { f1 (ppm) }\end{array}$ & -77.5 & -78.5 & -79.5 & -80.5 & -81.5 & -82.5 & -83.5 \\
\hline
\end{tabular}

31P NMR (212 MHz, CDCI3)

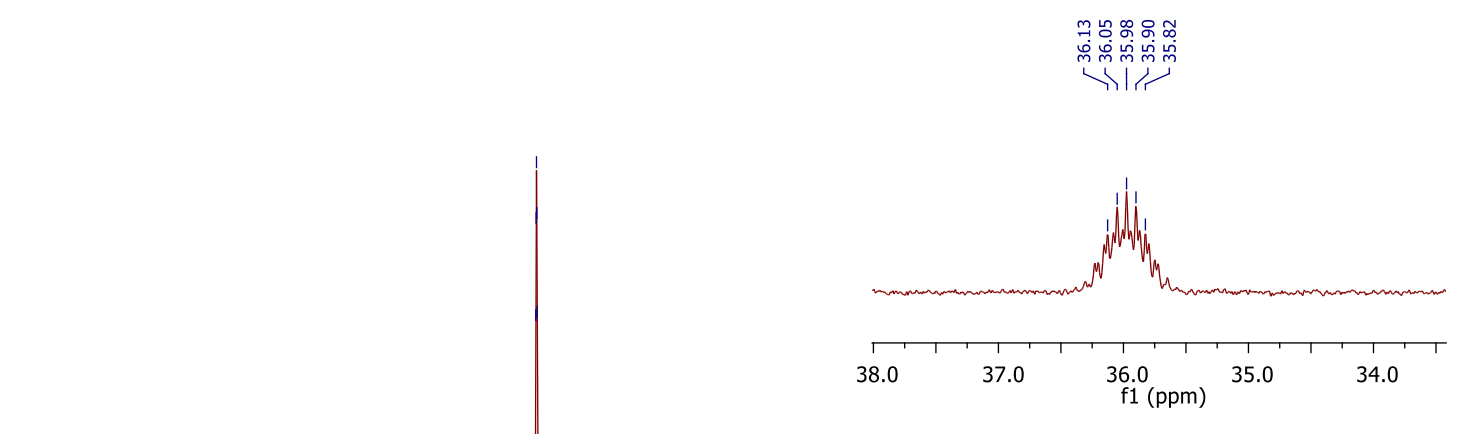

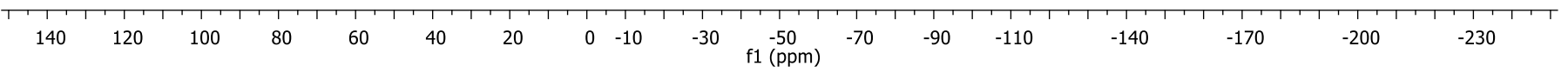


${ }^{1} \mathrm{H}-\mathrm{NMR}\left(300 \mathrm{MHz} ; \mathrm{CDCl}_{3}\right)$<smiles>[10BH]CN([10BH2])C(=O)CCCOC(I)[CH]CI</smiles>

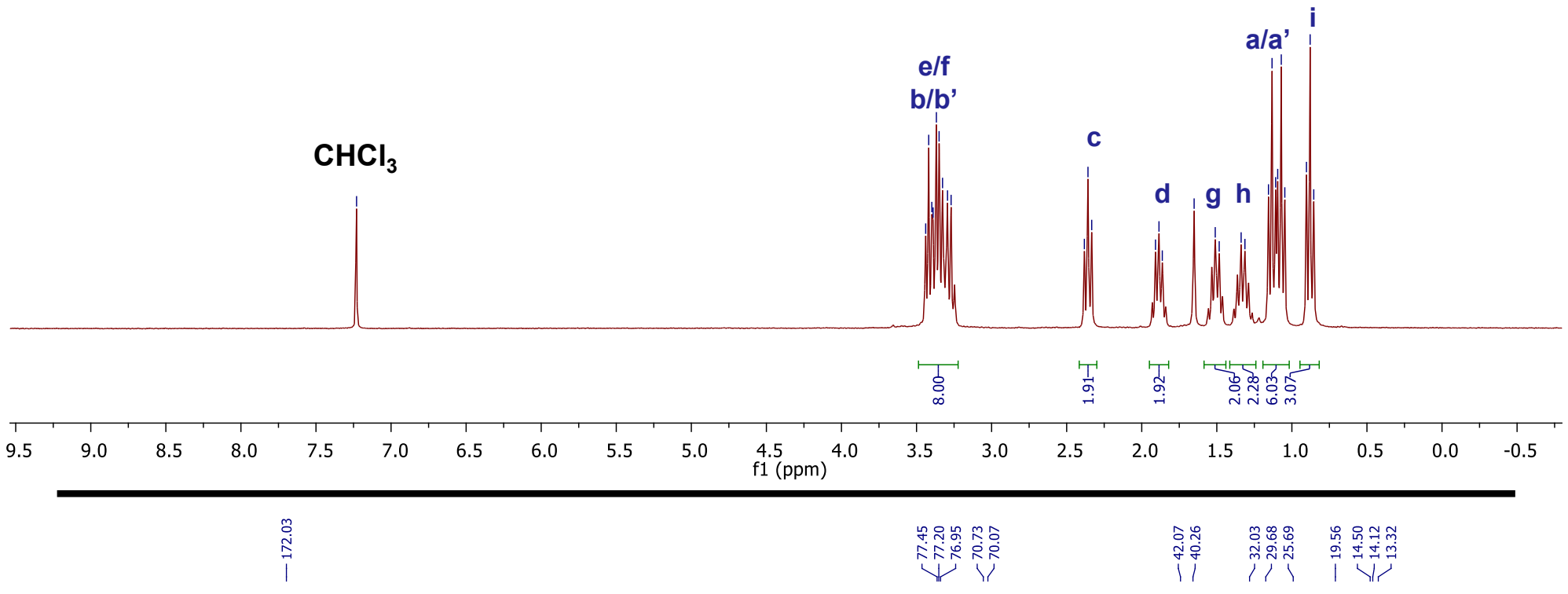

${ }^{13} \mathrm{C}-\mathrm{NMR}\left(126 \mathrm{MHz} ; \mathrm{CDCl}_{3}\right.$ )

$\mathrm{CHCl}_{3}$

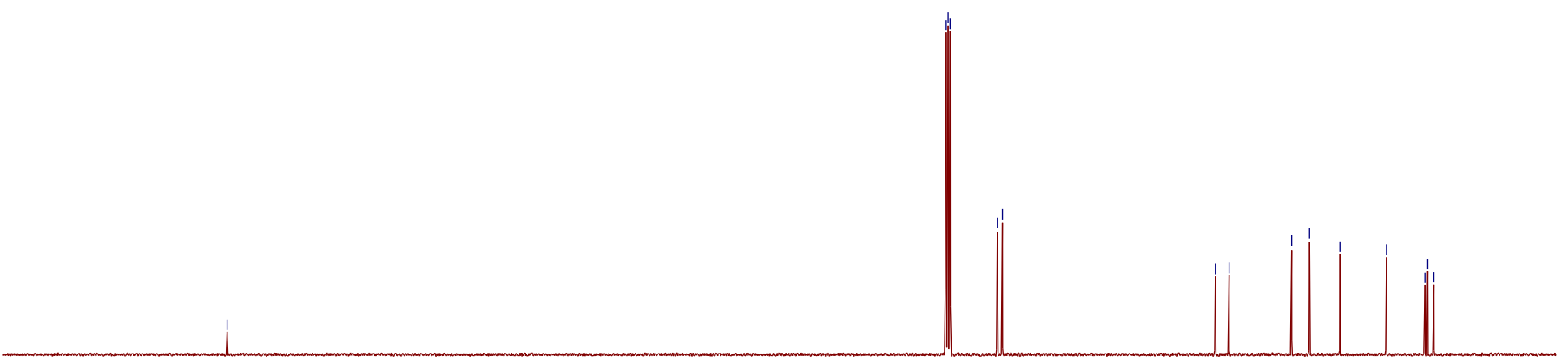

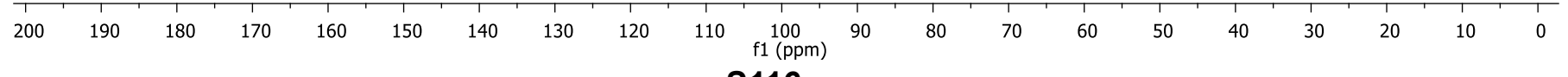


${ }^{1} \mathrm{H}-\mathrm{NMR}\left(300 \mathrm{MHz} ; \mathrm{CDCl}_{3}\right)$

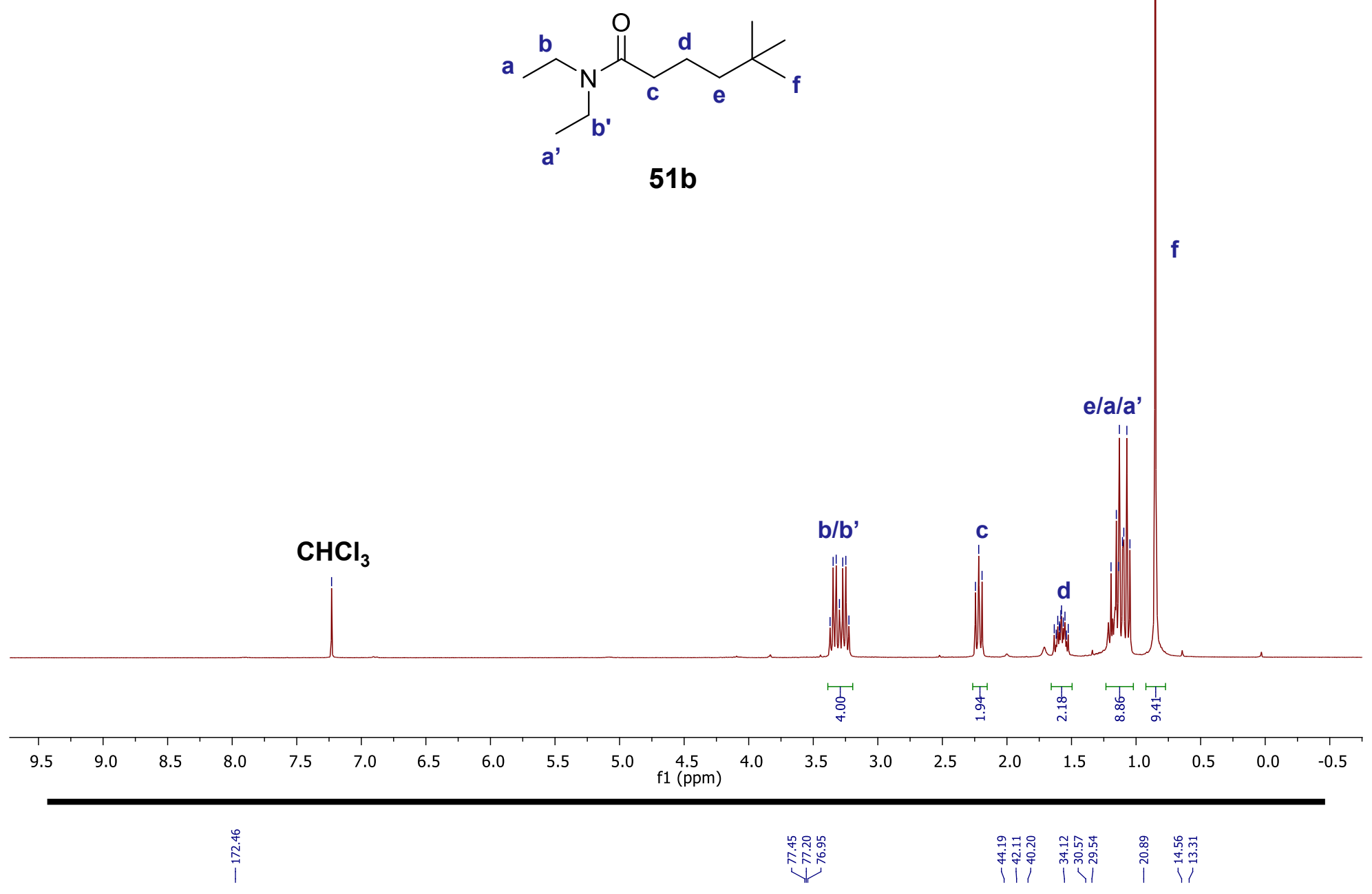

${ }^{13} \mathrm{C}-\mathrm{NMR}\left(126 \mathrm{MHz}^{\mathrm{CDCl}}{ }_{3}\right)$

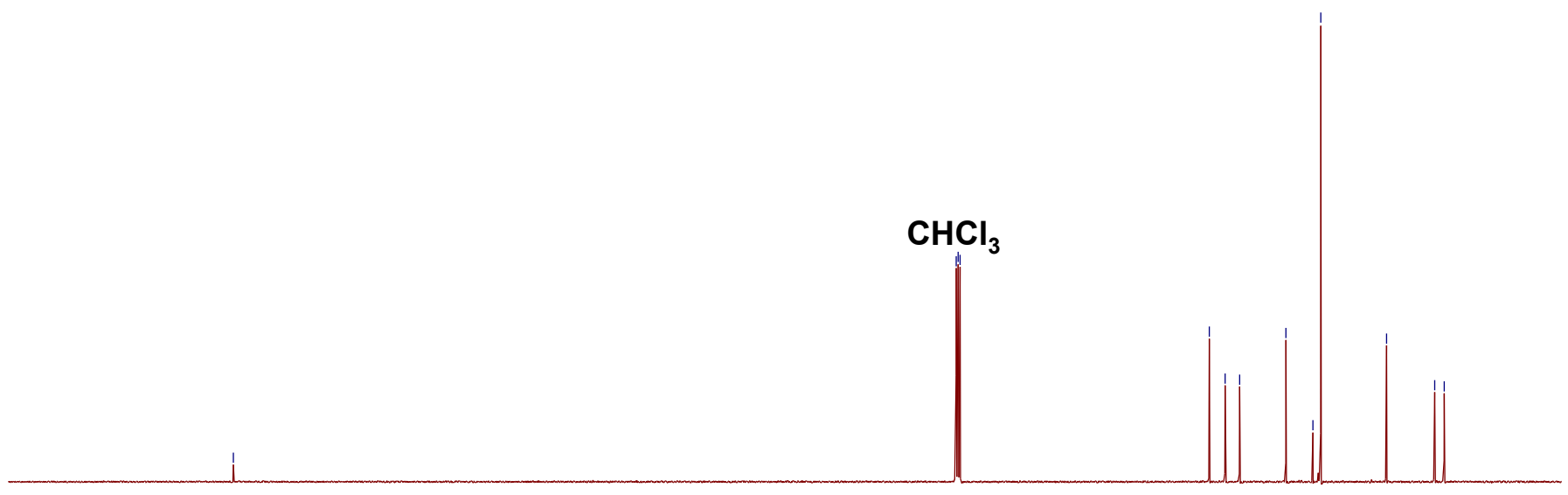

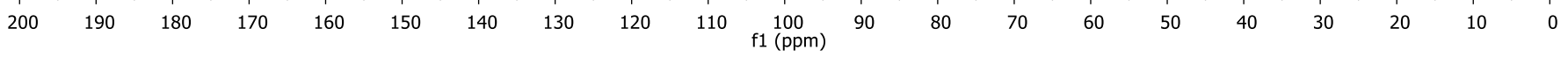


${ }^{1} \mathrm{H}-\mathrm{NMR}\left(300 \mathrm{MHz} ; \mathrm{CDCl}_{3}\right)$

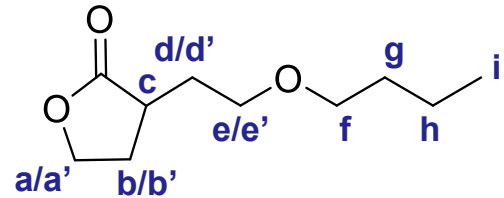

$52 a$

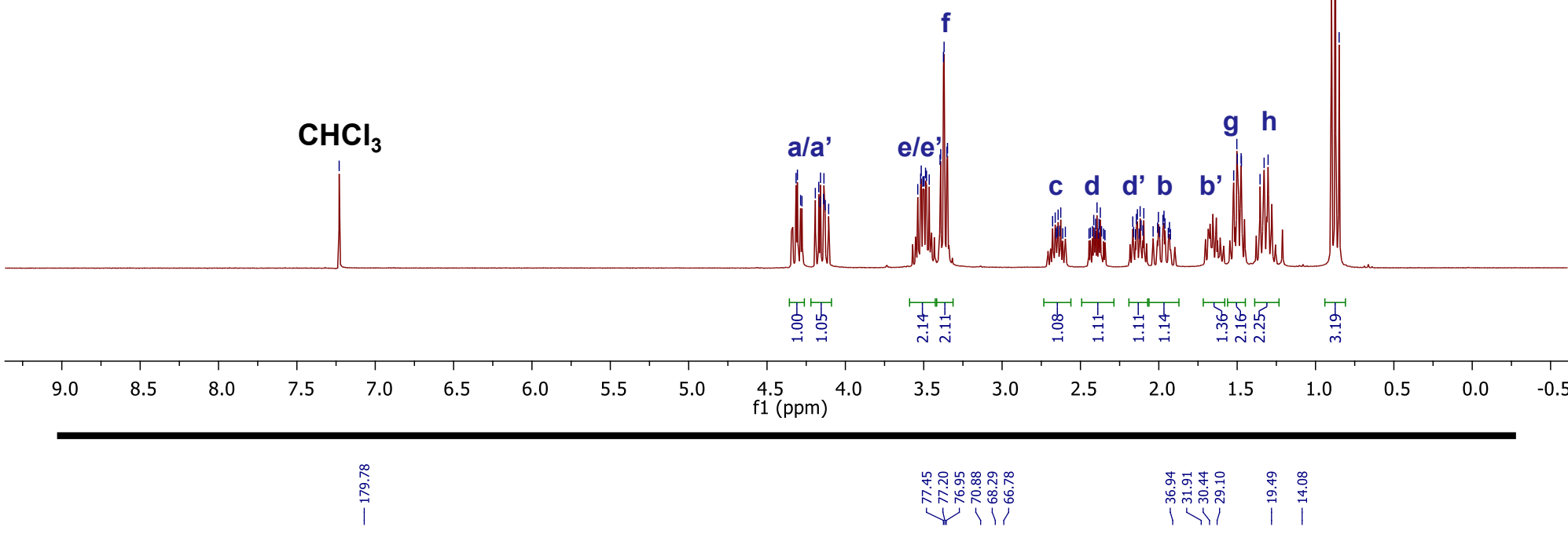

${ }^{13} \mathrm{C}-\mathrm{NMR}\left(126 \mathrm{MHz} ; \mathrm{CDCl}_{3}\right.$ )

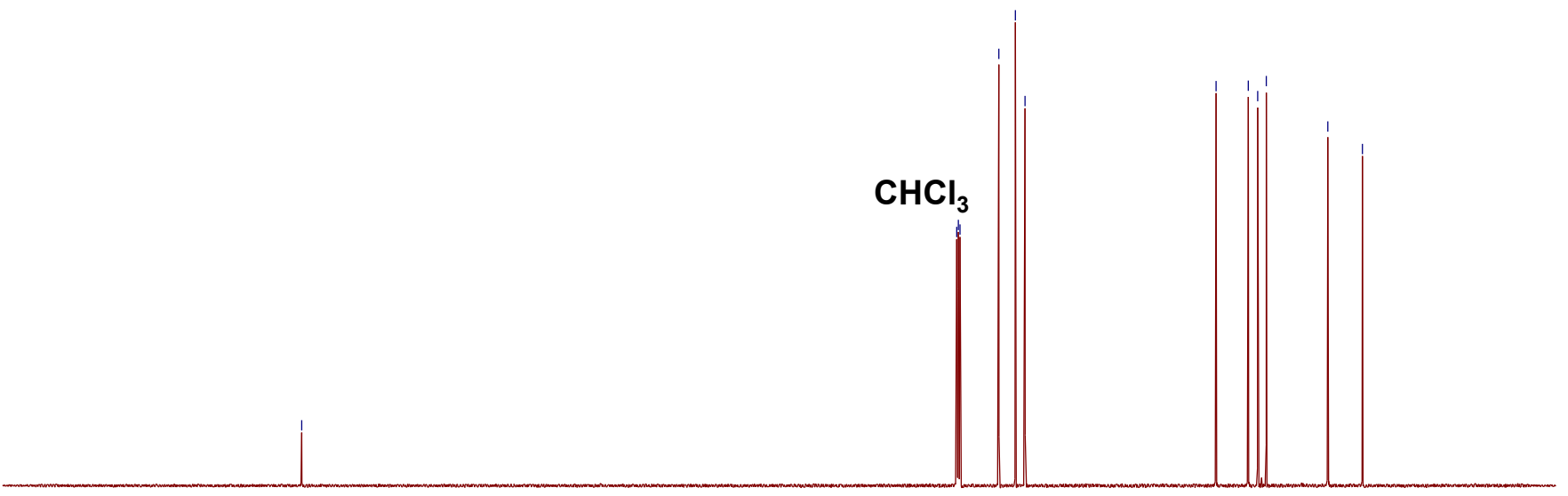

$\begin{array}{llllllllllll}220 & 210 & 200 & 190 & 180 & 170 & 160 & 150 & 140 & 130 & 120 & \begin{array}{l}110 \\ \mathrm{f} 1(\mathrm{ppm})\end{array}\end{array}$ 
${ }^{1} \mathrm{H}-\mathrm{NMR}$ (300 MHz; $\mathrm{CDCl}_{3}$ )

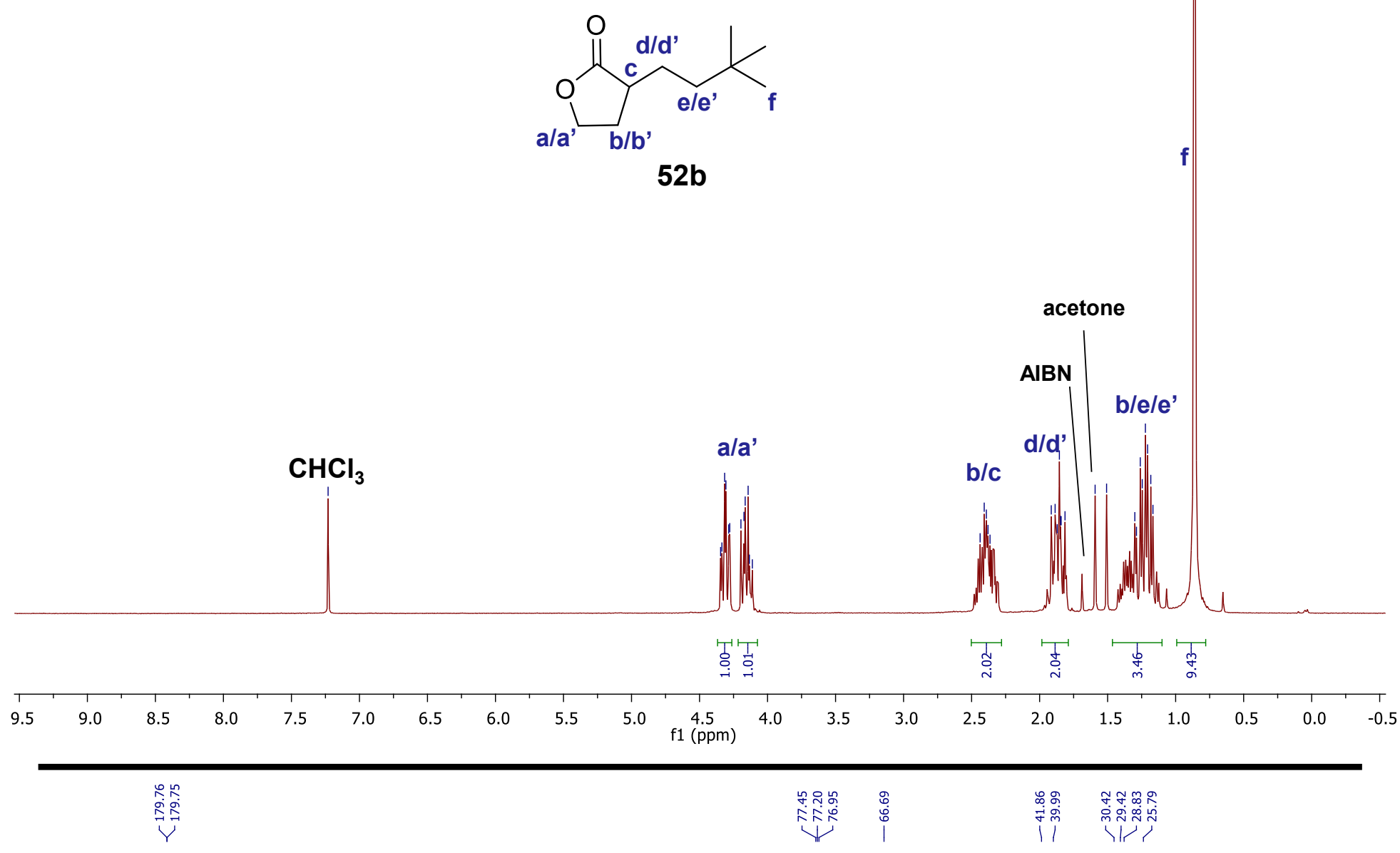

${ }^{13} \mathrm{C}-\mathrm{NMR}$ (126 MHz; $\mathrm{CDCl}_{3}$ )

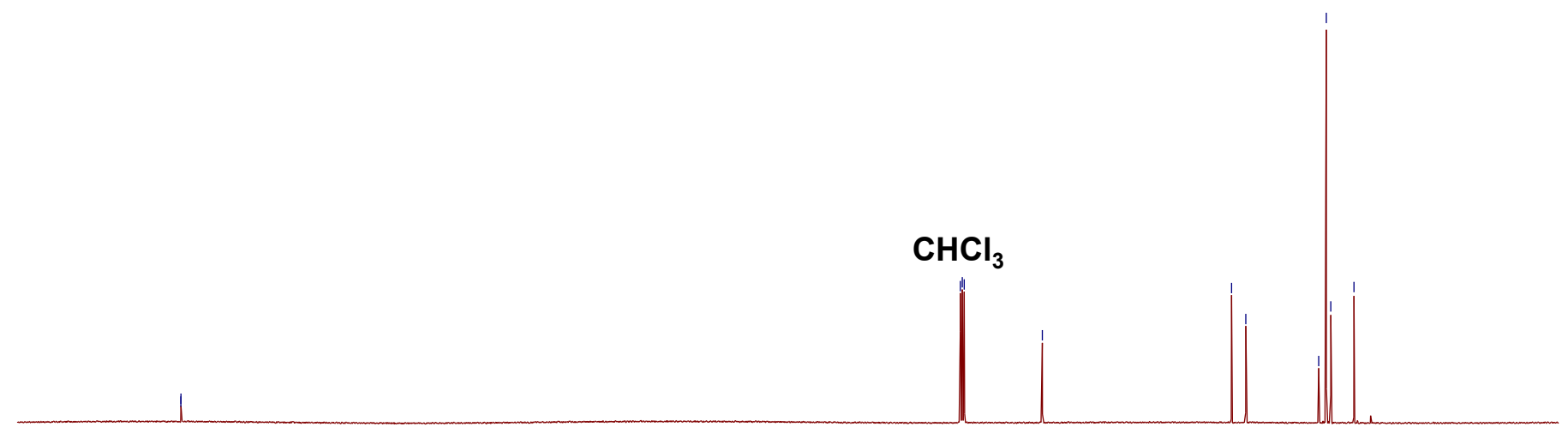

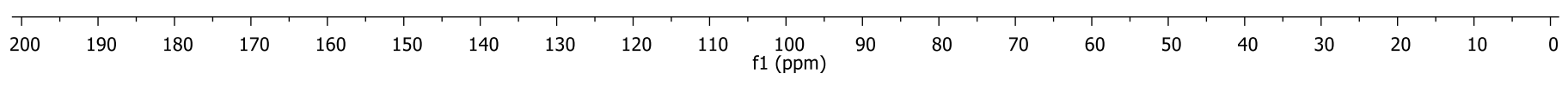

